\title{
Christian Lahusen,
}

Stephanie Schneider ( $\mathrm{Hg}$.)
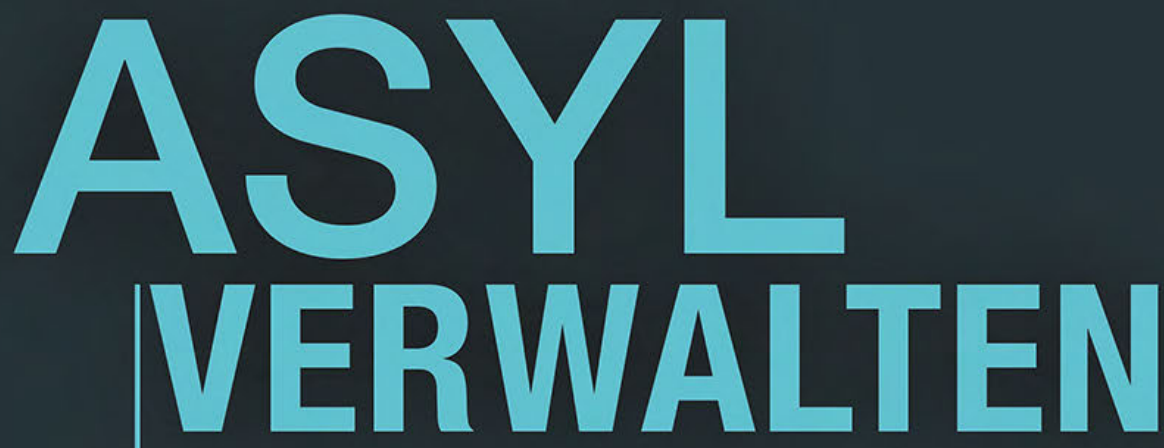

Zur bürokratischen Bearbeitung eines gesellschaftlichen Problems 
Christian Lahusen, Stephanie Schneider (Hg.)

Asyl verwalten 

Christian lahusen, Stephanie Schneider (Hg.)

\section{Asyl verwalten}

Zur bürokratischen Bearbeitung eines gesellschaftlichen Problems

[transcript] 
Gedruckt mit freundlicher Unterstützung der Deutschen Forschungsgemeinschaft (FOR1539/Teilprojekt 02).

\section{(c) $(\mathcal{9} \Theta \Theta$}

Dieses Werk ist lizenziert unter der Creative Commons Attribution-NonCommercial-NoDerivs 4.0 Lizenz (BY-NC-ND). Diese Lizenz erlaubt die private Nutzung, gestattet aber keine Bearbeitung und keine kommerzielle Nutzung. Weitere Informationen finden Sie unter https://creativecommons.org/licenses/by-nc-nd/4.o/deed.de/. Um Genehmigungen für Adaptionen, Übersetzungen, Derivate oder Wiederverwendung zu kommerziellen Zwecken einzuholen, wenden Sie sich bitte an rights@transcript-verlag.de

\section{(C) 2017 transcript Verlag, Bielefeld}

Die Verwertung der Texte und Bilder ist ohne Zustimmung des Verlages urheberrechtswidrig und strafbar. Das gilt auch für Vervielfältigungen, Übersetzungen, Mikroverfilmungen und für die Verarbeitung mit elektronischen Systemen.

\section{Bibliografische Information der Deutschen Nationalbibliothek}

Die Deutsche Nationalbibliothek verzeichnet diese Publikation in der Deutschen Nationalbibliografie; detaillierte bibliografische Daten sind im Internet über http://dnb.d-nb.de abrufbar.

Umschlaggestaltung: Kordula Röckenhaus, Bielefeld Druck: Majuskel Medienproduktion GmbH, Wetzlar

Print-ISBN 978-3-8376-3332-0

PDF-ISBN 978-3-8394-3332-4

Gedruckt auf alterungsbeständigem Papier mit chlorfrei gebleichtem Zellstoff. Besuchen Sie uns im Internet: http://www.transcript-verlag.de Bitte fordern Sie unser Gesamtverzeichnis und andere Broschüren an unter: info@transcript-verlag.de 


\section{Inhalt}

Asyl verwalten: Eine Einleitung

Christian Lahusen und Stephanie Schneider 17

\section{DIE POLITISCHE KONSTRUKTION DES \\ EUROPÄISCHEN ASYLVERWALTUNGSRAUMS}

Politikinstrumente in der europäischen Asylpolitik:

Zur Rolle von Experten und Expertise

Nina Amelung | 27

"Asyl fängt ja erst an, wenn er [der Flüchtling] wirklich hier ist « Der Wandel europäischer Visapolitik und seine Implikationen für den Zugang zu Asylverfahren in der EU

Lena Laube $\mid 55$

\section{ÜBER ASYLANTRÄGE ENTSCHEIDEN: \\ ZUR PRAXIS BEHÖRDLICHEN HANDELNS}

"Ohne 'ne ordentliche Anhörung kann ich keine ordentliche Entscheidung machen...» - Zur Organisation von Anhörungen in deutschen und schwedischen Asylbehörden

Stephanie Schneider und Kristina Wottrich | 81

Entscheiden über Asyl: Organisationssoziologische Überlegungen zum Zusammenspiel von Formalität und Informalität im österreichischen Asyl-Verwaltungsverfahren Julia Dahlvik | 117

Asyl-Verwaltung kraft Wissen: Die Herstellung von Entscheidungswissen in einer Schweizer Asylbehörde Laura Affolter | 145 


\section{Die Verwaltung von Asyl als umkämpfte Praxis}

Ausländerbehörden im dynamischen Feld der Migrationssteuerung Tobias G. Eule | 175

Die Entstehung rechtlicher Fallgeschichten in einem Übersetzungsprozess: Die Rechtsvertretung asylsuchenden Personen in einem schweizerischen Hilfswerk Johanna Fuchs $\mid 195$

Auseinandersetzungen über Abschiebungen: Handlungsoptionen in einem umkämpften Feld Carla Küffner | 223

Autorinnen und Autoren | 253 


\section{Asyl verwalten}

\section{Eine Einleitung}

\section{Christian LAHUSEn Und Stephanie SCHNEIDER}

\section{Einleitung}

Asylgewährung ist ein Gegenstand behördlicher Verwaltungsarbeit. Diese lapidare Beobachtung ist in den letzten Jahren in seiner ganzen Tragweite ins öffentliche Bewusstsein getreten. Mit Bezug auf Deutschland zeigt sich dies nicht zuletzt an den Auseinandersetzungen darüber, ob von einer >Flüchtlingskrise oder nicht vielmehr von einer >Verwaltungskrise $<$ gesprochen werden solle. Die Einschätzungen darüber, wie mit den nach Europa (zwangs-)migrierenden Menschen umzugehen sei, divergieren innerhalb wie zwischen Staaten, politischen Lagern und gesellschaftlichen Gruppen. Ein gesellschaftliches Problem stellt dies insofern dar, als zentrale Normen und Werte inzwischen öffentlichkeitswirksam zur Disposition gestellt werden (vgl. Brunkhorst 2016). Die Auseinandersetzungen drehen sich u.a. um die verbindliche Geltung und Auslegung grundlegender Normen des Völkerrechts und der Menschenrechte ebenso wie um die Abschaffung oder umso striktere Wiedereinführung von Grenzkontrollen. Im großen Kontinuum zwischen extremen Positionen scheint jedoch weitgehend Einigkeit darüber zu herrschen, dass die Mobilität, die Unterbringung und der Aufenthalt der Migrierenden irgendwie reguliert werden müsse und zwar mittels bürokratischer Verfahren.

Damit ist das Verwaltungshandeln weniger deutlich ins Fadenkreuz der öffentlichen Kontroversen geraten. Präsenter sind die Widersprüche und Grenzen der bestehenden Flüchtlings- und Asylpolitik der Europäischen Union und ihrer Mitgliedsstaaten. In diesem Zusammenhang konzentrierten sich die öffentlichen Debatten primär auf die dringlichsten Nöte und Probleme. Der Blick richtete sich auf die Gefahren der Fluchtrouten über das Mittelmeer und die vielen tragi- 
schen Schicksale, auf die Grenzen einer effektiven und flächendeckenden Hilfe für die Geflüchteten und auf die politischen Auseinandersetzungen zwischen den Regierungen, die eine zügige und adäquate Aufnahme und Unterbringung eher blockierten. Ein Themenbereich allerdings wurde und wird auf dieser Agenda stärker aus dem öffentlichen Interesse gedrängt: die Asylverfahren selbst. Gegenüber den politisch vermeintlich brisanteren Themen - etwa der Frage reffektiver`Grenzkontrollen, der Verteilung von Geflüchteten zwischen den Mitgliedsländern oder länderinternen >Belastungs-^ und 〉Obergrenzen ` - gerät die bürokratische Bearbeitung des Problems stärker aus dem Blickfeld. Dies ist erstaunlich, weil im und durch das administrative Verfahren die Differenzierungen vorgenommen werden, die letztlich über den Verbleib, den Status und die Gestaltung des Aufenthalts von Asylsuchenden entscheiden. Damit sind sie zum einen aus Sicht der Antragstellerinnen und Antragsteller von zentraler Bedeutung für ihr persönliches Schicksal. Zum anderen ist die Arbeit der Behörden folgenreich, insofern als darüber die Durchlässigkeit territorialer Grenzen reguliert und gesellschaftliche Regeln über Zugehörigkeit und Nicht-Zugehörigkeit beständig eingesetzt, verändert oder reproduziert werden. Über Anerkennungs- und Ablehnungsquoten wird mehr oder weniger explizit kommuniziert, welche Gruppen von Migrierenden des Schutzes >würdig s sind und welche nicht, woran wiederum eine Reihe weiterer Differenzierungen anknüpfen können. Gleichzeitig setzt sich >der Staatく mit Entscheidungen über Schutzgewährung auch nach außen immer wieder selbst als legitimer Akteur in Szene, wobei völkerrechtlichen und menschenrechtlichen Verpflichtungen Genüge getan werden muss, die einem wie auch immer verstandenen snationalen Interesse` Grenzen setzen. Insgesamt bewegen Asylbehörden sich damit im Spannungsfeld konfligierender Anforderungen und Ansprüche von gesamtgesellschaftlicher Bedeutung, die im Verwaltungshandeln beständig kleingearbeitet werden müssen.

Nun wäre es vermessen zu behaupten, Asylverwaltungen und Asylverfahren wären eine `black-box`, über die wir kaum etwas in der Öffentlichkeit mitbekämen. Ganz ohne Zweifel wird wiederkehrend und kontrovers darüber berichtet, wenn die bürokratische Prozessierung von Asylanträgen hakt. Immer wieder wird über erhöhte Verfahrensdauern, Bearbeitungsrückstaus und mangelnde Ausstattung der Behörden mit Personal und Ressourcen geklagt, die ein zügiges Abarbeiten verhindern. Berichte thematisieren auch Einzelschicksale mit ihren Fluchtbiographien, ihren Erfahrungen mit den Asylbehörden und den Folgen abschlägiger Asylbescheide. Aber die eigentliche Praxis der Asylverwaltungen, die Kernarbeit und Routinetätigkeiten, bleiben von diesem öffentlichen Interesse weitestgehend unberührt. 
Der vorliegende Sammelband möchte einen Beitrag zu diesem wenig erforschten Themenbereich liefern. Angesichts der Dringlichkeit der Probleme und der Geschwindigkeit, mit der die administrativen Verfahrensweisen immer wieder reformiert, verändert, improvisiert werden, scheint dies ein gewagtes Unterfangen. Wir plädieren jedoch dafür, einen Schritt zurückzutreten und den aufgeheizten medialen und politischen Debatten mit einem sozialwissenschaftlichen Blick zu begegnen, der sich der Thematik mit einer größeren Distanz und Unaufgeregtheit zuwendet, ohne die konkreten Zwänge, Probleme und Nöte der Betroffenen aus dem Auge zu verlieren. Insbesondere möchte der Band eine Reihe von Fragen klären, die das Verwaltungshandeln selbst betreffen. Welchen Zwängen sind die Mitarbeiterinnen und Mitarbeiter ausgesetzt und wie werden sie den vielfältigen rechtlichen, administrativen und persönlichen Anforderungen an ihre Arbeit gerecht? Wie kommen sie zu Entscheidungen in den Einzelfällen, und welchen Kriterien folgen sie dabei? Welche Arten von Beziehungen unterhalten die Behörden mit den Antragstellerinnen und Antragstellern und wie werden diese reguliert oder organisiert? Welche Rolle spielen andere Organisationen (bspw. Rechtsberatungen, Nichtregierungsorganisationen) mit Blick auf die Erstellung und Überprüfung von Bescheiden? Der Vergleich des Verwaltungshandelns in mehreren europäischen Ländern (Deutschland, Österreich, Schweden oder die Schweiz) eröffnet ein differenziertes Bild, das sowohl deutliche Gemeinsamkeiten offenbart als auch interessante Unterschiede.

Den Fokus auf die Behördenmitarbeiterinnen und -mitarbeiter zu legen, bedeutet dabei nicht, die Lage der Asylsuchenden zu ignorieren. Denn eine Analyse des Verwaltungshandelns hilft auch, die Anforderungen, Zumutungen und Problemlagen mit zu reflektieren, mit denen sich geflüchtete Menschen konfrontiert sehen. Bescheide, die über das Schicksal von Einzelpersonen entscheiden, werden bürokratisch hergestellt, weshalb eine Analyse dieses >Herstellungsprozesses` gleichzeitig auch eine >Aufklärung< über die Lage der Antragstellerinnen und Antragsteller ist. Eine wissenschaftliche Aufarbeitung des Verwaltungshandelns ist zudem auch deshalb geboten, weil wir über die Arbeitssituation der Mitarbeiterinnen und Mitarbeiter der Asylbehörden Klarheiten gewinnen sollten. Dies scheint uns umso dringlicher, als es gerade das Personal der Asylbehörden ist, das in den medialen Debatten - nicht nur in Deutschland - angesichts langer Verfahrensdauern und strittiger Einzelfallentscheidungen wiederholt in den Fokus der Kritik gerät. Die dem Verfahren inhärenten strukturellen (und damit auch schon vor der \Kriseく wirksamen) Widersprüche und Schwierigkeiten, mit denen die Mitarbeiterinnen und Mitarbeiter zu kämpfen haben, werden dagegen selten öffentlich thematisiert, was nicht zuletzt in der oft begrenzten Transparenz des Verwaltungshandelns begründet liegt. 
Im deutschsprachigen Raum gilt diese Wissenslücke auch für die sozialwissenschaftliche Auseinandersetzung mit dem Thema. Während die srefugee studies ২ v.a. im angelsächsischen Raum ein seit Längerem etabliertes Forschungsfeld sind, beginnt sich eine eigenständige `Flüchtlingsforschung $\$ in Deutschland gerade erst zu entwickeln. Bisherige Studien haben zudem eine meist rechtsoder politikwissenschaftliche Ausrichtung und beschäftigen sich primär mit der Makroebene des Asylrechts und der Asylpolitik (für viele andere siehe Bast 2011, 2014; Bungenberg/Giegerich/Stein 2016; Engelmann 2014; Müller 2010; anders aber: Ellermann 2006, 2009; Schammann 2015). Andere, eher soziologisch angelegte Forschungsarbeiten befassen sich mit den Auswirkungen der Bürokratie auf die Lebenswirklichkeit von Asylsuchenden (vgl. Täubig 2009; Fritsche 2012), den Folgen rechtlicher und bürokratischer Klassifizierungen aus ungleichheitssoziologischer Perspektive (vgl. Scherschel 2015; Pichl 2016; Scherr 2015) oder untersuchen die Interaktionen bei - im Gegensatz zum administrativen Verfahren öffentlichen - Gerichtsverhandlungen (vgl. Arndt 2015). Die für die Forschung mindestens ebenso interessante Frage nach der Verwaltungswirklichkeit und dem konkreten Handeln der Verwaltungsmitarbeiterinnen und -mitarbeiter ist im deutschsprachigen Raum bislang kaum systematisch behandelt worden (wichtige Ausnahmen sind die Studien von Scheffer 2001; Probst 2012; Dahlvik 2014 und Eule 2014). Diese Forschungsanstrengungen möchten wir durch den Sammelband weiter voranbringen. Ausgangspunkt des Buches sind Diskussionen, die wir am Kongress der Deutschen Gesellschaft für Soziologie im Oktober 2014 in einer Gruppe von Forscherinnen und Forschern geführt hatten, die sich mit dem >Inneren< des Verwaltungshandelns befassen. Die Forschungsergebnisse, die wir in diesem Band versammelt haben, machen deutlich, dass das methodische und theoretische Arsenal der Sozialwissenschaften, insbesondere das der Organisations- und Verwaltungsforschung, in fruchtbarer Weise dafür genutzt werden kann, sowohl die Besonderheiten des Asylverfahrens herauszuarbeiten als auch das dort beobachtete berufliche Handeln als Handeln in staatlichen Verwaltungsbehörden allgemein zu verstehen.

\section{Asylverwaltungen im Fokus der soziologischen Forschung}

Im Rückgriff auf die lange Tradition der Erforschung bürokratischer Organisationen innerhalb der Soziologie zeigt sich, dass die Probleme, mit denen Sachbearbeiterinnen und Sachbearbeiter im Asylbereich konfrontiert sind, keineswegs immer außergewöhnlich sind. Im Gegenteil: Viele der dort beobachtbaren Prob- 
lematiken und Dilemmata sind auf typische und altbekannte Charakteristika öffentlicher Verwaltungen zurückzuführen (vgl. Lipsky 2010). Drei dieser Spannungen und Dilemmata sind ausgemacht worden. Erstens ist auch für Asylverwaltungen beobachtet worden, dass die Arbeit einem zunehmenden Prozess der Standardisierung unterworfen ist, der Folge der wachsenden rechtlichen Regulierung, des neuen Managerialismus mit seiner Outputorientierung und den elektronischen Workflowsystemen mit ihren neuen Steuerungs- und Kontrollmöglichkeiten ist. Dieser Standardisierungsprozess kollidiert im Arbeitsalltag mit der Notwendigkeit von Handlungs- und Entscheidungsspielräumen im Einzelfall (vgl. Bovens/Zouridis 2002; Dunkerley et al. 2005; Rosenberger/König 2012). Gleichzeitig kann die Nutzung und Ausdeutung von Entscheidungsspielräumen seitens der Verwaltungsmitarbeiterinnen und -mitarbeiter auch eine Ungleichbehandlung von `Klientinnen Maynard-Moody/Musheno 2000, 2012; Brodkin 2008).

Diese Spannung ist mit zwei anderen eng verbunden. Zweitens müssen Behördenmitarbeiterinnen und -mitarbeiter den Produktivitätsanforderungen einer administrativen Massenabfertigung gerecht werden, zugleich aber eine den professionellen Ansprüchen zufolge `guteく Einzelfallprüfung durchführen, die bei negativem Bescheid auch noch gerichtsfest ist (vgl. Sorensen und Sorensen 1974; Gilboy 1991; Loyens/Maesschalck 2010). Und drittens sind auch Statusund Machtbeziehungen innerhalb der Behörden von Relevanz, denn für den Arbeitsalltag suchen Entscheider und Entscheiderinnen praktische Handlungsautonomie innerhalb von hierarchischen Steuerungs- und Kontrollstrukturen aufrecht zu erhalten (vgl. Triandafyllidou 2003; Bastien 2009; Evans 2011). Verwaltungshandeln auf dem sstreet-level ist folglich mit widersprüchlichen Anforderungen und Problemlagen konfrontiert, die im Arbeitsalltag nicht leicht zu lösen sind. Das Personal entwickelt Handlungsroutinen und Wissensbestände, die nicht notwendigerweise den formalen Organisationsstrukturen und Arbeitsabläufen entsprechen (vgl. Lipsky 1984; Bastien 2009; Schittenhelm 2015), aber Handlungsspielräume eröffnen und das Arbeitspensum zu erledigen erlauben (vgl. Jordan/Stråth/Triandafyllidou 2003). Diese Praktiken und Wissensbestände sind im Wesentlichen auch das, was Neulinge während ihrer Einstiegsphase und der innerbehördlichen Sozialisation erlernen (vgl. Reichers 1987; Heyman 1995).

Bei der Erforschung öffentlicher Verwaltungen stand häufig das sich wandelnde Verhältnis zwischen Staat und Bürgerinnen und Bürgern (mancherorten umgedeutet in eines der Dienstleistung zwischen Verwaltung und `Kunden $\triangleleft)$ im Mittelpunkt des Forschungsinteresses. Bei der Asylverwaltung hingegen geht es zunächst um die Entscheidung darüber, wer überhaupt zum Kollektiv derer mit 
(über fundamentale Menschenrechte hinausgehenden) Rechtsansprüchen gehören soll (für Botschaftspersonal siehe Alpes/Spire 2014). Als >gatekeeper« und 'Grenzverwalter ist sie im Kernbereich hoheitlichen Verwaltungshandelns anzusiedeln.

Wie die in diesem Band versammelten Beiträge zeigen, spielen hier Interpretationen, Klassifikationen und Kategorisierungen eine Rolle, die häufig schon im Vorfeld des >bureaucratic encounters« vorgenommen werden. $\mathrm{Zu}$ nennen sind hier beispielsweise Visabkommen (Laube in diesem Band), die Definition vermeintlich >sicherer Herkunftsländer und die Erfassung, Verteilung und Nutzung biometrischer Daten (Amelung in diesem Band). Solche Vorannahmen können die Interaktionen zwischen Asylsuchenden und den Mitarbeiterinnen und Mitarbeitern in den für erstinstanzliche Entscheidungen über Asylanträge verantwortlichen Behörden in erheblichem Maße vorstrukturieren. Der dort (nicht) verliehene Schutzstatus wiederum ist von wesentlicher Bedeutung für die Beziehungen zwischen Behörde und gesellschaftlicher `Umwelt` (Zivilgesellschaft, Politik, Gerichte, Medien u.a.) einerseits, (ehemaligen) Antragstellerinnen und Antragstellern und anderen staatlichen Behörden andererseits. Insofern als anerkannte oder abgelehnte Antragstellerinnen und Antragsteller zum Subjekt einer Reihe staatlicher Interventionen (bspw. was `Integrationsleistungen`, die Durchsetzung von Ausreiseaufforderungen usw. betrifft) werden können, wird der einmal verliehene Status zur wichtigen Grundlage nachfolgender Interaktionen und Auseinandersetzungen (siehe Eule, Fuchs und Küffner in diesem Band). Mit Entscheidungen über Schutzgewährung werden damit folgenreiche und im Kern moralische Bewertungen vorgenommen (vgl. Fassin 2005; Codó 2011).

Neben grundlegenden Gemeinsamkeiten mit anderen Verwaltungszweigen weist die Arbeit der Asylbehörden Besonderheiten auf, die für ein Studium des street-levels besonders lehrreich und ergiebig sind. Die Erstellung sachlich und administrativ richtiger Bescheide ist zwar ein Kennzeichen aller antragprüfenden Verwaltungen, aber in wenigen anderen Bereichen sind die Voraussetzungen hierfür so schwierig wie im Falle des Asyls. Bescheide sollen sachlich richtig sein, obschon den Behörden zuweilen grundlegende Informationen und Dokumente fehlen - u.a. auch zu Namen, Alter und Herkunft der antragstellenden Person. Die Mitarbeiterinnen und Mitarbeiter der Behörden müssen eindeutige Entscheidungen fällen, aber ohne eine ausreichend klare Sachlage zu besitzen, weshalb die entscheidungsrelevanten Fakten und die darauf begründete Eindeutigkeit im Verfahren erst generiert werden müssen (vgl. Probst 2011; siehe auch Dahlvik und Affolter in diesem Band). Im Bereich der Asylverwaltung kann eine mikrosoziologische Forschung dem Personal deshalb besonders gut dabei zu- 
schauen, wie Bürokratien Fälle konstruieren, Produktivität organisieren und bürokratisches Handeln legitimieren (siehe Schneider/Wottrich in diesem Band).

Aber noch ein anderer Aspekt des Arbeitsalltags in Asylverwaltungen ist besonders lehrreich für die Erforschung staatlicher Bürokratien. In administrativer Hinsicht nämlich muss das Behördenpersonal rein bürokratisch, d.h. ohne Ansehen der Person - >sine ira et studio< - entscheiden, obwohl die Interaktionen zwischen Behörden und Antragstellenden hochgradig emotional und normativ aufgeladen sind (siehe Fuchs und Küffner in diesem Band). Einerseits bleibt die emotionale Distanz des Behördenpersonals gegenüber den Antragstellerinnen und Antragstellern angesichts der dramatischen Lebensgeschichten und der weitreichenden Folgen (insb. abschlägiger) Bescheide brüchig (vgl. James/Killick 2012; Fassin 2005). Andererseits wird die Glaubwürdigkeit der antragstellenden Person zu einem ebenso wichtigen Referenzpunkt bei der Fallbearbeitung und Entscheidungsfindung, wie die zusammengetragene, aber doch lückenhafte und unsichere Sachlage (siehe Dahlvik und Affolter in diesem Band). In beiderlei Hinsicht ist der street-level damit konfrontiert, dass die Fallbearbeitung und Entscheidungsfindung kaum ohne Ansehen der Person, ohne Mitgefühl, Wut oder Leidenschaft vollzogen werden kann (vgl. Wettergren 2010). Immer wieder haben Untersuchungen dabei die zentrale Rolle des behördlichen Misstrauens, der >culture of disbelief^(vgl. Jubany 2011; Good 2015; Probst 2012; Affolter in diesem Band), im Umgang mit Asylanträgen hervorgehoben. Den Mitarbeiterinnen und Mitarbeitern der Asylverwaltungen können wir damit ebenfalls dabei zusehen, wie sie die Handlungslogik und das Selbstverständnis bürokratischer Organisationen gegenüber den Zumutungen der Komplexität der sozialen Welt der Antragstellerinnen und Antragsteller zu reproduzieren und zu legitimieren suchen.

\section{Asylverwaltung als komplexer Handlungs- zusammenhang: Politik, Gesellschaft und istreet-level bureaucracy،}

Der vorliegende Sammelband reiht sich damit in eine Reihe von Studien zur Asylverwaltungsforschung ein, die die Komplexitäten der Sachbearbeitung zum Gegenstand haben. ${ }^{1}$ Die hier versammelten Beiträge bestätigen den Ertrag einer

$1 \mathrm{Zu}$ diesen Studien gehören bspw. Alpes/Spire 2014; Dahlvik 2014; Eule 2014; Gibb/Good 2013; Good 2015; Hamlin 2014; James/Killick 2012; Jubany 2011; 
solchen Fragestellung. Sie eint ein Interesse an einer mikroanalytischen Betrachtungsweise administrativen Handelns. Die Mehrzahl von ihnen basiert auf qualitativen empirischen Studien, die sich ethnographischer Methoden bedienen; das präsentierte Material besteht aus Interviews, Akten und Beobachtungsdaten. Im Fokus steht das praktische Tun der Mitarbeiterinnen und Mitarbeiter, die für den Vollzug der auf europäischer oder nationaler Ebene beschlossenen Gesetze und Politiken verantwortlich sind. Der fallanalytische und ländervergleichende Ansatz erlaubt es zudem, theoretische, methodologische und empirische Fragen nach den Eigenlogiken und Spannungsmomenten, den Grenzziehungen, wechselseitigen Beeinflussungen und Dynamiken zwischen den Behörden und ihrer gesellschaftlichen Umwelt zu beantworten. Über Fragen der Implementation hinausgehend möchte der Sammelband damit insgesamt zu einem tiefergehenden Verständnis bürokratischen Handelns im speziellen Bereich des Asyls und der dafür relevanten Kontextbedingungen beitragen.

Das Buch ist in drei Teile gegliedert. Teil I versammelt Beiträge, die die politische Konstruktion eines europäischen Asylverwaltungsraums aus unterschiedlichen Perspektiven beleuchten. Nina AmELUNG befasst sich in ihrem Aufsatz mit Politikinstrumenten in der europäischen Asylpolitik und fokussiert dabei insbesondere die Rolle von Experten und Expertise. Sie unterscheidet zwischen vier verschiedenen Typen solcher Politikinstrumente: den rechtsverbindlichen Regelungen (Verordnungen und Richtlinien), den freiwilligen Kooperationsmaßnahmen (Umsiedlungsprogramme und ihre Anreizstrukturen), den indirekten Steuerungsmaßnahmen über >capacity building` (etwa durch Schulungsprogramme) oder über >monitoring^ (bspw. durch Berichterstattung und Datenbanken). In einem Überblick über die Entwicklung europäischer Politikinstrumente zeigt sie auf, wie diese in der Praxis der Mitgliedsstaaten unterschiedlich genutzt - oder nicht genutzt - werden. Aus sozialkonstruktivistischer Sicht werden am Beispiel des Instruments EURODAC auch die nicht-intendierten (Neben-)Folgen europäischer Asylpolitik analysiert und kritisch hinterfragt. Politikinstrumente sind für die Autorin deshalb von Bedeutung, da sie Vorstellungen, Wissensbestände und Praktiken etablieren, die das Verhältnis von regulierenden Institutionen organisieren und lenken. Bei der Nutzung und Verbreitung solcher Instrumente können sehr heterogene Akteursgruppen aus unterschiedlichen gesellschaftlichen Teilbereichen einbezogen werden, womit der Einflussbereich derselben wesentlich erweitert werden kann. An EURODAC lässt sich dabei veran-

Mountz 2010; Probst 2012; Schoultz 2014; Spire 2007; Wettergren/Wikström 2014; Whyte 2015. 
schaulichen, wie expertisebasierte Instrumente einen Regelungsbereich naturalisieren, neutralisieren und damit auch entpolitisieren.

LENA LAUBE interessiert sich ebenfalls für die politisch-regulatorische Konstruktion von Asyl, hier vor allem durch eine Untersuchung der Zuwanderungsoptionen, die die Europäische Union durch ihre Migrations- und Asylpolitik eröffnet oder verbaut. Ihre konkrete Fragestellung zielt auf die engen Verknüpfungen zwischen dem Feld des Asyls und jenem der Visapolitik. Aus diachroner Perspektive macht sie im Wandel von Visapolitiken seit dem zweiten Weltkrieg drei Phasen aus, die sie mit Blick auf ihre Folgen für Asylsuchende analysiert. Fungierte während der ersten Phase die Praxis der Visabefreiung noch vornehmlich als symbolischer Akt, als »Geschenk« und außenpolitisches Signal in diplomatischen Beziehungen zwischen Staaten, so ist die zweite Phase durch vermehrte Restriktionen und die (Wieder-)Einführung der Visumspflicht für bestimmte Herkunftsländer gekennzeichnet. In dieser Phase entwickeln sich engere Verkopplungen zwischen Visa- und Asylpolitik; zugleich wird das Politikfeld auf europäischer Ebene stärker vergemeinschaftet. LAUBE zufolge lässt sich in der aktuellen dritten Phase Visafreiheit v.a. als gewichtiges Pfand in den Verhandlungen der EU mit Nachbarländern verstehen, wie sich am Beispiel der Verhandlungen mit der Türkei eindrücklich zeigt. Die auf politischer Ebene beschlossenen Abkommen über Reisefreiheiten bzw. -einschränkungen können spürbare Auswirkungen auf die Asylfrage haben, denn mit der Visumsbefreiung geht oftmals die Annahme einher, dass in diesen Ländern keine Verfolgung drohe. Visapolitik erweist sich damit als konstitutiver Bestandteil eines europäischen Feldes der Verwaltung von Asyl.

Vor dem Hintergrund der europäischen Asyl- und Migrationspolitik werden in Teil II des Bandes die Behörden, die in erster Instanz über Asylanträge entscheiden, fokussiert. Hier geht es um Verwaltungshandeln an den >front-lines nationaler Asylbehörden. Die drei Beiträge eint die Frage danach, welche Wissensformen für Verwaltungspraktiken in spezifischen Kontexten relevant sind, welche Handlungsmuster die behördliche Praxis anleiten und wie Organisationsstrukturen und Arbeitsabläufe auf Entscheidungspraktiken einwirken.

Der Beitrag von STEPHANIE SCHNEIDER und KRISTINA WOTTRICH greift dafür zunächst die europäische Problemperspektive auf. Sie interessieren sich für die Frage, ob und in welcher Weise die eingespielten Arbeitsroutinen und Wissensbestände des street-levels von den Bemühungen der Europäischen Union um eine Harmonisierung der Asylverfahrenspraxis irritiert oder verändert werden. Ihre behördenvergleichende Untersuchung schwedischer und deutscher Asylbehörden richtet den Blick auf ein europäisches Schulungsprogramm für Asylsachbearbeiterinnen und -sachbearbeiter (das EASO-Training Curriculum) und auf dessen 
Aufnahme durch das Behördenpersonal. Am Beispiel der Anhörungspraxis zeigen die Autorinnen, dass die Anforderungen einer optimierten, sozialwissenschaftlichen Techniken entsprechenden Gesprächsführung, die im ETC propagiert werden, bei den Sachbearbeiterinnen und Sachbearbeitern durchaus auf offene Ohren stoßen, zumal die Methoden auch im Sinne einer Entlastung oder Arbeitserleichterung angewandt werden können. Die konkrete Aneignung und Anwendung des neuen Wissens erfolgt jedoch selektiv und mit Blick auf die Lösung konkreter, praktischer Probleme. Diese wiederum sind wesentlich durch die organisationalen Bedingungen, Leitbilder und Arbeitsroutinen geprägt. Hier arbeiten die Autorinnen unterschiedliche Verfahrensmuster und -stile heraus, da in schwedischen und deutschen Behörden die Fallbearbeitung tendenziell mehr Gewicht auf Mündlichkeit oder Schriftlichkeit, kollektive oder individuelle Zuständigkeit, Konsensorientierung oder Konflikthaftigkeit legt.

Die Asylverfahrenspraxis ist damit auf der lokalen Ebene zwar irritierbar, aber folgt im Wesentlichen den auf dem street-level etablierten Arbeitsroutinen und Wissensbeständen. Die Trägheit des eingespielten Verwaltungshandelns scheint vor allem an der Informalität der Arbeitsroutinen und Wissensbestände zu liegen, mit denen sich JULIA DAHLVIK in ihrem Beitrag befasst. Sie zeigt anhand ihrer ethnographischen Studie im österreichischen Bundesamt für Fremdenwesen und Asyl, dass der organisationssoziologische Forschungsstand in der Untersuchung von Asylbehörden fruchtbar eingesetzt werden kann, um zentrale strukturelle Dilemmata zu identifizieren, die das Arbeiten in der Asylbehörde kennzeichnen. Vor allem spielen die Dilemmata »Verantwortung vs. Distanzierung« und »Eindeutigkeit vs. Ungewissheit« im Asylverfahren eine besondere Rolle. Dies liegt zum einen daran, dass im Asylbereich mehr auf dem Spiel steht, als in den meisten anderen staatlichen Verwaltungsbereichen; zum anderen herrscht durch den Mangel an Beweisen ein besonders hohes Maß an Ungewissheit. DAHLVIK zeigt auf, wie in der alltäglichen Bearbeitung dieser Dilemmata Formalität und Informalität als soziale Ordnungsformen wechselseitig zum Tragen kommen. Während die alltäglichen sozialen Prozesse in der Behörde eher durch Informalität geregelt sind, durchlaufen die informellen Praktiken (mehr oder weniger erzwungene) Prozesse der Formalisierung, wie DAHLVIK am Beispiel der `Faktisierung « und der Verschriftlichung demonstriert. Der Beitrag verdeutlicht, wie die strukturellen Spannungen in der täglichen Arbeit über die soziale Konstruktion von Fakten im Zusammenspiel von Formalität und Informalität bearbeitet werden.

Diesem Erkenntnisinteresse folgend beschäftigt sich LAURA AFFOLTER in ihrem Beitrag ebenfalls mit der Herstellung von Entscheidungswissen. Aus wissenssoziologischer Perspektive befasst sie sich hierbei mit der Verfahrenswirk- 
lichkeit innerhalb der Direktion Asyl des schweizerischen Staatssekretariats für Migration (SEM). Aufbauend auf der Weberianischen Unterscheidung von Fachund Dienstwissen zeigt sie, wie im und durch das Asylverfahren verschiedene Wissensformen produziert und mobilisiert werden. Ihre ethnographischen Feldbeobachtungen zeigen, dass die Einschätzung von Wahrscheinlichkeiten für vergangene und zukünftige Verfolgung im Verfahren eine ganz entscheidende Rolle einnimmt. Die Mehrheit der ablehnenden Bescheide wird nicht mit fehlenden Voraussetzungen bezüglich der Flüchtlingseigenschaft, sondern mit der Unglaubhaftigkeit von Asylvorbringen begründet. Dies erklärt sich nach AFFOLTER v.a. durch das dem Verfahren inhärenten institutionelle Misstrauen und über die Art und Weise, wie im Asylverfahren Entscheidungswissen hergestellt wird. Sie zeigt, dass >Widersprüche « das meist verwendete Kriterium zur Feststellung von Unglaubhaftigkeit sind und dass das Personal hierfür Fragestrategien und Praktiken entwickelt und nutzt, um diese nachweisen zu können. Der Autorin zufolge müssen administrative Verfahren "praktische Gewissheit» schaffen, die sich auch für die Sachbearbeiterinnen und -sachbearbeiter als »gefühlte Sicherheit« eignet. Dadurch, dass dieses implizite Wissen nicht allgemein zugänglich ist, entfaltet das in der Behörde produzierte Entscheidungswissen seine Macht.

Nachdem der zweite Teil des Buches v.a. mit der Herstellung von administrativen Entscheiden im Asylverfahren betraut war und verdeutlicht hat, auf welchen Voraussetzungen die Praxis der erstinstanzlichen Asylvergabe beruht, geht es im dritten Teil darum, den Blick für die Beziehungen zwischen Asylbehörden und der gesellschaftlichen Umwelt zu öffnen. Deutlich wird dabei, dass die im administrativen Verfahren getroffenen Entscheidungen ihrerseits zum Gegenstand von Aushandlungen, Konflikten und Umdeutungen zwischen unterschiedlichen staatlichen und nicht-staatlichen Akteuren werden. Nichtregierungsorganisationen und Protestgruppen, Rechtsberaterinnen und -berater, Anwältinnen und Anwälte, Kommunen, die Medien und viele andere Akteure begleiten die administrative Bearbeitung der Asylanträge oder suchen diese über die jeweiligen Instanzen und Stadien des Verfahrens zu beeinflussen. Diese vielfältigen Beziehungen und Interaktionen verdeutlichen, dass die Handlungsfähigkeit und Effektivität des Verwaltungshandelns im Wesentlichen auch durch externe Faktoren geprägt wird. Die hier versammelten Beiträge folgen dem Verfahren und offenbaren die Bedeutung der gesellschaftlichen Umwelt bei der Fallbegleitung, der gerichtlichen Überprüfung und der drohenden Abschiebung.

Das Verhältnis zwischen Verwaltung und Zivilgesellschaft thematisiert TOBIAS EULE. Er befasst sich mit kommunalen Ausländerbehörden in Deutschland, die im Bereich des Asyls die wichtigsten, nicht mit dem Asylantrag selbst verknüpften Aufgaben übernehmen (bspw. Aufenthaltserlaubnis, Abschiebung 
oder Einbürgerung). Im Zentrum seines Beitrags steht die Frage nach den Beziehungen zur lokalen Zivilgesellschaft, denn in der Diskussion wird davon ausgegangen, dass dieses Verhältnis ein primär konfliktives sei. Seine längeren ethnographischen Feldforschungen zeichnen diesbezüglich ein erstaunliches Bild, denn das Personal der Ausländerbehörden und Akteure der lokalen Zivilgesellschaft unterhalten sehr oft persönliche und vertrauensvolle Beziehungen und arbeiten informell recht eng zusammen. Hinzu kommen ein größeres Verständnis für die jeweils andere Seite und eine größere Anerkennung der jeweiligen Arbeit. Obschon die zivilgesellschaftlichen Akteure immer noch >kritische Begleiter sind, so ist das Verhältnis heute weniger konfliktiv und primär kooperativ oder unterstützend. Hierfür macht EULE drei Entwicklungen verantwortlich. Zunächst verweist er auf die Liberalisierung des Migrationsrechts, denn die stärkere Orientierung am Migrationsmanagement schafft mehr Spielräume für positive Bescheide. Sodann verweist er auf den staatlichen Ausbau von integrationsförderlichen Maßnahmen, die für viele zivilgesellschaftliche Akteure neue Betätigungsfelder schaffen. Und schließlich sieht er eine Öffnung der restriktiven Politik vieler Kommunen durch die Orientierung am Leitbild der $>$ Willkommensbehörden<.

Das Interesse an zivilgesellschaftlichen Organisationen teilt JOHANNA FUCHS. In ihrem Beitrag konzentriert sie sich aber auf die nichtstaatlichen Hilfswerke, die sich auf Rechtsberatung und -vertretung in Fragen des Asyl- und Ausländerrechts spezialisiert haben. Ihre ethnographischen Feldforschungen hat sie in der Schweiz durchgeführt und möchte auf dieser Grundlage klären, in welcher Form diese Akteure in die rechtliche Konstruktion von >Fällen〈 involviert sind und welchen Zwängen eine erfolgreiche Konstruktion administrativ anschlussfähiger Fallgeschichten unterliegt. Sie geht davon aus, dass Rechtsberaterinnen und -berater im Wesentlichen die Aufgabe der `Übersetzung « - zwischen der Lebenswirklichkeit der Asylsuchenden und den Kriterien des Rechts - übernehmen. Die Praxis der Rechtsberatung bewegt sich dabei an der Schnittstelle zwischen den Behörden und den Asylsuchenden. Um aus dem, was die Asylsuchenden in der Beratung erzählen, eine verfahrenstaugliche Geschichte zu machen, müssen Fallgeschichten kreativ an bestehende rechtliche Kategorien und entscheidungsrelevante Kriterien angepasst und plausibel gemacht werden. Fuchs stellt die Ergebnisse einer ethnographischen Fallanalyse dar und zeigt, wie im Verlauf von sechs Monaten drei unterschiedliche Fallgeschichten hergestellt wurden, die sich allerdings nie vollständig in die jeweilige rechtliche Kategorie integrieren ließen. Sie zeigt auch, wie Rechtsberatung vielfältige, zum Teil widersprüchliche Fallgeschichten generiert, die ihrerseits in ein Netz vorangegangener oder parallel verlaufender `Übersetzungen` einfließen. Obschon Rechtsbe- 
ratung explizit eine Vermittlungs- und Übersetzungsfunktion übernimmt, spielen diese Akteure somit auch eine Rolle als >Gatekeeper` zum gerichtlichen Verfahren.

CARLA KÜFFNER schließlich demonstriert, dass auch in letzter Instanz ablehnende Entscheidungen noch Teil gesellschaftlicher Auseinandersetzungen sind. Am Beispiel der (Nicht-)Durchsetzung von Abschiebungen in Österreich und Deutschland untersucht sie, über welche Handlungsspielräume ausgesuchte Akteure zu unterschiedlichen Zeitpunkten des Abschiebeprozesses jeweils verfügen. Von juristischen Interventionen über Aushandlungen, öffentlichen Protest und zivilen Ungehorsam bis hin zu widerständigen Körperpraktiken analysiert sie Abschiebungen als umkämpften Prozess. Die Handlungsmacht der Akteure ist darin ein gewichtiger, nicht zu vernachlässigender Faktor. Während der Ausgang der Auseinandersetzungen nicht im Vorhinein feststeht, bleibt am Ende doch meist die Abschiebbarkeit bestehen und ist die Nichtabschiebung fragil und temporär. In ihrem Ausblick stellt KÜFFNER daher die Frage nach der gesellschaftlichen Funktion von Abschiebbarkeit und Nichtabschiebung. Wenn Abschiebungen nicht durchgesetzt werden, handelt es sich dabei nicht schlicht um ein Kontrolldefizit, sondern auch um Abschiebepolitik als Steuerungsinstrument. Denn im Wissen um prinzipielle Abschiebbarkeit werden rechtliche Unsicherheit und Prekarität hergestellt und darüber die »Regierung der Durchlässigkeit« erleichtert, was sich bspw. auch auf den informellen Arbeitsmarkt auswirken kann. Zweitens gilt es Küffner zufolge, Nichtabschiebung aus der Perspektive der Autonomie der Migration, von den »Kämpfen der Migration« aus zu verstehen und damit auch die »Instabilitäten der Hegemonie« zu erfassen.

Die Beiträge dieses Sammelbands bestätigen auf vielfältige Weise, wie voraussetzungsvoll der administrative Vollzug im Bereich des Asyls ist. Politik und Recht können zwar bindend festlegen, welche Rechte Asylsuchende haben und welche Verfahren zur Überprüfung der Anspruchsberechtigung einzuhalten sind. Aber letztendlich liegt es am Personal auf dem street-level, den Arbeitsauftrag zu erfüllen. Wie wir gesehen haben, sind die Mitarbeiterinnen und Mitarbeiter in ein Geflecht von persönlichen und materialen Beziehungen eingebunden, die als Last und Chance zugleich in Erscheinung treten. Asyl- und dienstrechtliche Bestimmungen bieten entlastende Entscheidungshilfen bei der Fallbearbeitung, stehen aber unter Umständen einer adäquaten Bewertung des konkreten Einzelfalls im Wege. Datenbanken und Computerprogramme können Wissenslücken schließen und Unsicherheit reduzieren, aber überbrücken damit nicht die Verständigungsprobleme zwischen Asylsuchenden und Behördenpersonal. Rechtsberaterinnen und -berater und zivilgesellschaftliche Organisationen können zwar 
Behörden bei der Begleitung und Betreuung von Asylsuchenden unterstützen, aber auch Verfahrensabläufe erschweren oder torpedieren.

Die Beiträge dieses Sammelbandes geben beredtes Zeugnis dafür ab, dass das Verwaltungshandeln zwar in den Händen einzelner Behördenmitarbeiterinnen und -mitarbeiter liegt, aber ein kollektiver - zum Teil kooperativer, zum Teil konfliktiver - Handlungszusammenhang ist, der weit über den Schreibtisch des betreffenden Personals hinausreicht. Dieser durchweg kollektive Handlungszusammenhang, in den der street-level eingebunden ist, wird auch für die relative Trägheit der Verwaltungspraxis verantwortlich sein. Denn diese Praxis fußt auf eingespielten und gemeinsam getragenen Wissensbeständen und Arbeitsroutinen. Veränderungen können nicht punktuell ansetzen, ohne die Verwobenheit administrativer Wissensbestände und Arbeitsroutinen zu adressieren. Für all diejenigen, die Verwaltungsapparate reformieren, in die eine oder andere Richtung steuern oder im Sinne ihrer Anliegen bewegen möchten, stellt diese Trägheit ein Ärgernis dar. Es gehört zur Herrschaft der street-level Bürokratie, dass sie die Zwänge der bestehenden Verwaltungspraxis perpetuiert - gegenüber ihrem eigenen Personal wie auch gegenüber ihrer Umwelt.

Für die Asylsuchenden ist dieses Ärgernis besonders bedrückend, denn sie werden einer Verwaltungspraxis unterworfen, deren Spielregeln sie nicht kennen und kaum beeinflussen können. Das physische Gewaltmonopol wird für sie unmittelbar greifbar, denn die Behörden entscheiden ja auch über Wohnorte, Mobilität und Verbleib im Land. Gleiches gilt aber auch für das symbolische Gewaltmonopol, denn die Legitimierung bürokratischer Herrschaft als ein Betrieb sachlich und rechtlich eindeutiger Entscheidungsfindung delegitimiert zugleich all diejenigen Asylsuchenden, deren Ansprüche sie abweist.

Allerdings sind Bürokratien nicht immun gegen Wandel. Veränderungen der Verwaltungspraxis können aber nur Wirkung zeigen, wenn sie nicht allein an den formalen Organisationsstrukturen und Arbeitsabläufen ansetzen, sondern an den informellen Wissensbeständen und Handlungsroutinen selbst. Die im Asylverfahren angelegten Widersprüche, Konflikte und Zumutungen werden aber nicht verschwinden, da sie zu den Strukturelementen der Verwaltungswirklichkeit gehören.

\section{Literatur}

Alpes, Maybritt Jill/Spire, Alexis (2014): »Dealing with Law in Migration Control: The Powers of Street-level Bureaucrats at French Consulates«, in: Social \& Legal Studies 23, S. 261-274. 
Arndt, Sophie (2015): »Ambivalente Rechtssubjektivität. Zur Position Asylsuchender in der gerichtlichen Interaktion«, in: Zeitschrift für Rechtssoziologie 35, S. 117-141.

Bast, Jürgen (2011): Aufenthaltsrecht und Migrationssteuerung, Tübingen: Mohr Siebeck.

- (2014): »Solidarität im europäischen Einwanderungs- und Asylrecht«, in: Michèle Knodt/Anne Tews (Hg.), Solidarität in der EU, Baden-Baden: Nomos.

Bastien, Joëlle (2009): »Goal ambiguity and informal discretion in the implementation of public policies: the case of Spanish immigration policy«, in: International Review of Administrative Sciences 75(4), S. 665-685.

Bovens, Mark/Zouridis, Stavros (2002): »From Street-Level to System-Level Bureaucracies: How Information and Communication Technology is Transforming Administrative Discretion and Constitutional Control«, in: Public Administration Review 62(2), S. 174-184.

Brodkin, Evelyn Z. (2008): »Accountability in Street-Level Organizations«, in: International Journal of Public Administration 31, S. 317-336.

Brunkhorst, Hauke (2016): »Neugründung der Union. Lehren aus der >Flüchtlingskrise««, in: Culture, Practice and European Policy 1, S. 1-13.

Bungenberg, Marc/Giegerich, Thomas/Stein, Torsten (Hg.) (2016): Asyl und Migration in Europa - rechtliche Herausforderungen und Perspektiven, Sonderband der Zeitschrift für europarechtliche Studien, Baden-Baden: Nomos.

Codó, Eva (2011): »Regimenting discourse, controlling bodies: Disinformation, evaluation and moral categorization in a state bureaucratic agency«, in: Discourse \& Society 22, S. 723-742.

Dahlvik, Julia (2014): Administering Asylum Applications, Unveröffentlichte Dissertation, Universität Wien.

Dunkerley, David/Scourfield, Jonathan/Maegusuku-Hewett, Tracey/Smalley, Nina (2005): »The experiences of frontline staff working with children seeking asylum«, in: Social Policy and Administration 39(6), S. 640-652.

Ellermann, Antje (2009): States against migrants. Deportation in Germany and the United States, Cambridge/New York: Cambridge University Press.

- (2006): " Street-level Democracy? How immigration bureaucrats manage public opposition«, in: West European Politics 29, S. 287-303.

Engelmann, Claudia (2014): »Informelles Regieren in der europäischen Asylpolitik«, in: Zeitschrift für Vergleichende Politikwissenschaft 8, S. 169-192.

Eule, Tobias (2014): Inside Immigration Law. Migration Management and Policy Application in Germany, Farnham: Ashgate. 
Evans, Tony (2011): »Professionals, managers and discretion: Critiquing streetlevel bureaucracy«, in: British Journal of Social Work 41(2), S. 368-386.

Fassin, Didier (2005): „Compassion and Repression: The Moral Economy of Immigration Policies in France«, in: Cultural Anthropology 20, S. 362-387.

Fritsche, Andrea (2012): »Zeit, Macht, Flüchtlinge. Und Flüchtlinge machen Zeit - Konzeptionen biografischer Zeiten im Asylkontext«, in: SWSRundschau 52, S. 362-288.

Gibb, Robert/Good, Anthony (2013): »Do the Facts Speak for Themselves? Country of Origin Information in French and British Refugee Status Determination Procedures«, in: International Journal of Refugee Law 25, S. 291-322.

Gilboy, Janet A. (1991): »Deciding who gets in: Decision making by immigration inspectors «, in: Law and Society Review 25(3), S. 571-600.

Good, Anthony (2015): ») The Benefit of the Doubt< in British Asylum Claims and International Cricket«, in: Daniela Berti/Anthony Good/Gilles Tarabout (Hg.), Of doubt and proof. Ritual and legal practices of judgment, S. 119-140.

Hamlin, Rebecca (2014): Let me be a refugee. Administrative justice and the politics of asylum in the United States, Canada, and Australia, New York: Oxford University Press.

Heyman, Josia McC. (1995): »Putting power in the anthropology of bureaucracy: The immigration and naturalization service at the Mexico-United States border«, in: Current Anthropology 36(2), S. 261-287.

James, Deborah/Killick, Evan (2012): »Empathy and Expertise: Case Workers and Immigration/Asylum Applicants in London«, in: Law \& Social Inquiry 37, S. 430-455.

Jordan, Bill/Stråth, Bo/Triandafyllidou, Anna (2003): »Comparing cultures of discretion«, in: Journal of Ethnic and Migration Studies 29(2), S. 373-395.

Jubany, Olga (2011): »Constructing truths in a culture of disbelief: Understanding asylum screening from within«, in: International Sociology 26, S. 74-94.

Lipsky, Michael (2010): Street-level bureaucracy. Dilemmas of the individual in public services, 30th anniversary expanded edition, New York: Russell Sage Foundation.

— (1984): »Bureaucratic Disentitlement in Social Welfare Programs«, in: Social Service Review 58, S. 3-27.

Loyens, Kim/Maesschalck, Jeroen (2010): »Toward a Theoretical Framework for Ethical Decision Making of Street-Level Bureaucracy. Existing Models Reconsidered«, in: Administration \& Society 42(1), S. 66-100. 
Maynard-Moody, Steven/Musheno, Michael (2000): »State Agent or Citizen Agent: Two Narratives of Discretion«, in: Journal of Public Administration Research and Theory 10, S. 329-358.

- (2012): »Social Equities and Inequities in Practice: Street Level Workers as Agents and Pragmatists «, in: Public Administration Review 72, S. 16-23.

Mountz, Alison (2010): Seeking asylum. Human smuggling and bureaucracy at the border, Minneapolis: University of Minnesota Press.

Müller, Doreen (2010): Flucht und Asyl in europäischen Migrationsregimen, Göttingen: Universitätsverlag Göttingen.

Pichl, Maximilian (2016): »Diskriminierung von Flüchtlingen und Geduldeten«, in: Albert Scherr/Aladin El-Mafaalani/Emine Gökcen Yüksel (Hg.), Handbuch Diskriminierung, Wiesbaden: Springer Fachmedien, S. 1-15.

Probst, Johanna (2011): »Entre faits et fiction. L'instruction de la demande d'asile en Allemagne et en France«, in: Cultures \& conflits, S. 63-80.

- (2012): Instruire la demande d'asile. Étude comparative du processus décisionnel au sein de l'administration allemande et francaise, Unveröffentlichte Dissertation, Université de Strasbourg/Philipps-Universität Marburg.

Reichers, Arnon E. (1987): »An interactionist perspective on newcomer socialization rates«, in: Academy of Management Review 12(2), S. 278-287.

Rosenberger, Sieglinde/König, Alexandra (2012): »Welcoming the unwelcome: The politics of minimum reception standards for asylum seekers in Austria«, in: Journal of Refugee Studies 25(4), S. 537-554.

Schammann, Hannes (2015): „Wenn Variationen den Alltag bestimmen. Unterschiede lokaler Politikgestaltung in der Leistungsgewährung für Asylsuchende«, in: Zeitschrift für Vergleichende Politikwissenschaft 9, S. 161-182.

Scheffer, Thomas (2001): Asylgewährung. Eine ethnographische Verfahrensanalyse, Stuttgart: Lucius \& Lucius.

Scherr, Albert (2015): »Wer soll deportiert werden? Wie die folgenreiche Unterscheidung zwischen den >wirklichen Flüchtlingen, den zu Duldenden und den Abzuschiebenden hergestellt wird«, in: Soziale Probleme 26(2), S. 151-170.

Scherschel, Karin (2015): »Zwischen universellen Menschenrechten und nationalstaatlicher Kontrolle: Flucht und Asyl aus ungleichheitssoziologischer Perspektive«, in: Soziale Probleme 26(2), S. 123-136.

Schittenhelm, Karin (2015): »Asylsuchende im Blickfeld der Behörden. Explizites und implizites Wissen in der Herstellung von Asylbescheiden in Deutschland «, in: Soziale Probleme 26(2), S. 137-150. 
Schoultz, Isabel (2014): »Seeking Asylum and Residence Permits in Sweden: Denial, Acknowledgement, and Bureaucratic Legitimacy«, in: Critical Criminology 22, S. 219-235.

Sorensen, James E./Sorensen, Thomas L. (1974): »The conflict of professionals in bureaucratic organizations«, in: Administrative Science Quarterly 19(1), S. 98-106.

Spire, Alexis (2007): »L'asile au guichet«, in: Actes de la recherche en sciences sociales 169 , S. 4-21.

Täubig, Vicki (2009): Totale Institution Asyl. Empirische Befunde zu alltäglichen Lebensführungen in der organisierten Desintegration, Weinheim: Juventa.

Triandafyllidou, Anna (2003): »Immigration policy implementation in Italy: Organizational culture, identity processes and labor market control«, in: Journal of Ethnic and Migration Studies 29(2), S. 257-297.

Wettergren, Åsa (2010): »Managing unlawful feelings. The emotional regime of the Swedish migration board«, in: International Journal of Work Organisation and Emotion 3, S. 400-419.

Wettergren, Åsa/Wikström, Hanna (2014): »Who Is a Refugee? Political Subjectivity and the Categorisation of Somali Asylum Seekers in Sweden«, in: Journal of Ethnic and Migration Studies 40, S. 566-583.

Whyte, Zachary (2015): »In Doubt: Documents as Fetishes in the Danish Asylum System«, in: Daniela Berti/Anthony Good/Gilles Tarabout (Hg.), Of doubt and proof. Ritual and legal practices of judgment, S. 141-161. 
Die politische Konstruktion des europäischen Asylverwaltungsraums 



\section{Politikinstrumente in der europäischen Asylpolitik: Zur Rolle von Experten und Expertise ${ }^{1}$}

NinA AmELUnG

\section{Einleitung}

Die Politikinstrumentenforschung findet zunehmend Verbreitung (vgl. Hood 2007; Lascoumes/Le Galès 2007), auch in Bezug auf europäische Politikverfahren. Allerdings ist das bislang vor allem in einzelnen Politikbereichen, wie in der Umwelt-, Forschungs-, Gleichstellungs- und gemeinsamen Außen- und Sicherheitspolitik der Fall (vgl. Bruno/Jacquot/Mandin 2006; Menon/Sedelmeier 2010; Voß 2010; Voß/Simons 2014) und noch nicht systematisch für den Bereich der Asylpolitik. Konventionelle Forschung zu Politikinstrumenten befasst sich mit der Auswahl von Politikinstrumenten und deren Implementierung. Sie nimmt dabei eine funktionalistische Perspektive ein, in der Politikwerkzeuge der Problemlösung im Politikprozess dienen und hinsichtlich ihrer Eignung und Effizienz zur Problemlösung untersucht werden (vgl. Linder/Peters 1990). Im Kontrast dazu wird hier eine sozialkonstruktivistische Sicht eingenommen, die Implikatio-

1 Ich bedanke mich bei Christian Lahusen, Stephanie Schneider, Tillmann Loehr, Eric Töpfer und Corinna Amelung für hilfreiche Kommentare zu früheren Versionen dieses Kapitels. Dieses Kapitel wäre ohne die Kenntnisse, die ich in jahrelanger Zusammenarbeit mit Kolleginnen und Kollegen der Innovation in Governance Research Group unter Leitung von Jan-Peter Voß an der TU Berlin und der gemeinsamen theoretischen und empirischen Arbeit zu Politikinstrumenten erlangt habe, nicht entstanden. Alle verbliebenen Fehler habe ich alleinig zu verantworten. 
nen dieser funktionalen Instrumentierung offen legt und kritisch hinterfragt. Instrumentierung produziert eigene Effekte auch jenseits der ihr zugeschriebenen Ziele der Problemlösung (vgl. Hood 2007; Lascoumes/Le Galès 2007). Das resultiert daraus, dass Politikinstrumente als Träger von Vorstellungen über die Rolle und das Verhältnis von regulierenden Institutionen in der EU oder eines Staates zur Gesellschaft verstanden werden. Eine sozialkonstruktivistische Perspektive interessiert sich dafür, in welcher Weise Politikinstrumente kondensiertes Wissen über die Art von Regierungstätigkeiten konstituiert, das als legitim und effektiv erachtet wird.

Den Mehrwert einer solch konstruktivistischen Perspektive auf Dynamiken der Instrumentierung für die Migrationsforschung sehen Trauner und Wolff (2014) vor allem darin, dass diese Perspektive Einflüsse auf die Migrationspolitik offen legt, die sonst unentdeckt bleiben können. Ihr eigener Beitrag zur europäischen migrationsorientierten Public Policy Forschung (Trauner/Wolff 2014) beschäftigt sich mit einer spezifischen Frage. Sie analysieren, wie Politikinstrumente, die als Instrumente der EU-Außenpolitik bereits etabliert sind, sich über die Zeit in konstanter Interaktion mit Drittstaaten und internationalen Organisationen weiterentwickeln. Auf diesem Weg werden die spezifischen Einflüsse von Drittstaaten und internationalen Organisationen über die Mitgestaltung von solchen Instrumenten auf die Entwicklung der außenpolitischen Komponente der EU-Migrationspolitik aufgezeigt. Diese Einflüsse, so ihr Ausgangspunkt, ist die Forschungslücke, die lange Zeit vernachlässigt wurde und die sie auf diesem Weg adressieren.

Wenn der Blick auf die Nebeneffekte der Instrumentierung gerichtet wird, wird sichtbar, welchen Akteuren und welchen Zwecken Instrumente tatsächlich dienlich sind. Verfolgt man die Entwicklung und Etablierung von Instrumenten im zeitlichen Verlauf, wird erkennbar, wie Instrumente nicht zwingend oder nicht ausschließlich zur Erreichung der ihnen zugeschriebenen Ziele nützen, sondern dass sie die Legitimierung von anderen Zwecken manifestieren. Trauner und Wolff lassen sich für die Übertragung auf migrationspolitische Instrumente durch Beispiele der neueren umweltpolitischen Instrumente in der EU inspirieren (vgl. Halpern 2010; Trauner/Wolff 2014). Dort wird dieser Ansatz in Bezug auf neu eingeführte marktbasierte Instrumente sowie freiwillige Vereinbarungen genutzt. Bei diesen Beispielen zeigt sich bei der Anwendung einer solchen Perspektive, dass häufig der innovative Charakter dieser Instrumente in den Vordergrund gestellt wird. Dies geschieht allerdings vor allem um insbesondere die Legitimation der umweltpolitischen Kompetenz der EU-Institutionen zu unterstreichen. Dabei werden jedoch häufig Instrumente von internationaler oder nationaler Ebene nur übernommen. Zusätzlich zeigt sich in solchen Analysen, dass diese 
Instrumente vor allem bestimmten Akteursgruppen dienen, die über sie Zugang zur Umweltpolitik der EU erhalten.

In der soziologischen Asylforschung hat die in angrenzenden Forschungsfeldern existierende oder sich etablierende Forschung zu Politikinstrumenten bislang noch keinen Niederschlag gefunden. Das ist umso erstaunlicher, da Forschung zu einzelnen europäischen asylpolitischen Instrumenten bereits ausgeprägt stattfindet. ${ }^{2}$ In diesen Untersuchungen werden aber Konzepte der sozialkonstruktivistischen Politikinstrumentenforschung nicht genutzt. Dabei liegt es nahe, an diese in der europäischen Asylforschung anzuschließen, wie ich in diesem Beitrag erläutern werde. Meine Überlegungen entwickle ich auch vor dem Hintergrund, dass andere an sozialkonstruktivistische Politikinstrumentenforschung anschlussfähige Konzepte, wie zum Beispiel zu den Themen Expertise und Experten im Politikprozess, durchaus Beachtung in der Asylforschung finden. Forschung zu diesen Themen hat dazu beigetragen, die Rolle von verschiedenen Wissenskulturen in Asylverwaltungen für die Legitimation von politischen Entscheidungen in EU-Mitgliedsstaaten als auch auf EU-Ebene offenzulegen und die Beeinflussung von asylpolitischen Normen durch die Einbeziehung von Experten und bestimmten Formen der Wissensproduktion zu erkennen (vgl. Boswell 2012; Boswell/Geddes 2011). Darüber hinaus haben andere Perspektiven dazu beigetragen, die Einflüsse von Praktiken von Experten(netzwerken) aus asylpolitischen, verwaltungstechnischen und exekutierenden sozialen (Professions-)Gruppen (inklusive Administration, Polizei und Militär, aber auch Medien, NGOs, Aktivisten) mit ihren spezifischen Expertisen aus ihren jeweiligen sozialen (Arbeits-)Welten zur Strukturierung einzelner Politiken und Politikfelder besser zu verstehen. Anhand dessen lassen sich beispielsweise die Auswirkungen des europäischen sicherheitspolitischen Feldes und der dort vorherrschenden Paradigmen von innerer Sicherheit, Abwehr von illegaler Migration und Ausbau von Kontrolle und Überwachung auf die Ausgestaltung und Bedeutungszuschreibung von asylpolitischen Maßnahmen nachvollziehen (vgl. Bigo 2014).

Durch die Untersuchung der Entwicklung und Implementierung von europäischen Politikinstrumenten - wie zum Beispiel EURODAC als Instrument zur Datenerfassung und zum Abgleich von Fingerabdrücken von Asylsuchenden kann das Entstehen und Zusammenwirken von spezifischen Expertennetzwerken

2 Beispiele dafür sind Instrumente wie die Datenbank EURODAC zur Erfassung von Fingerabdrücken von Asylsuchenden (vgl. Ploeg 1999; Schuster 2011), sowie Instrumente der Asylstatistik (vgl. Mouzourakis 2014) oder das Instrument des sicheren Herkunftsstaatenkonzeptes (vgl. Byrne/Shacknove 1996; Goodwin-Gill 1992; Gurzu 2012; Hunt 2014). 
zur Koordination des Instrumentes verfolgt werden. Solche Expertennetzwerke bestehen aus heterogenen Expertengruppen wie politischen Entscheidungsträgern, Asylverwaltung, Sicherheitstechnik, Polizei etc. Dabei lässt sich neben der den Expertengruppen jeweils eigenen epistemischen Rahmung die Verhandlung von gemeinsamen Deutungen von Problemen, Zwecken und Funktionen des Instrumentes beobachten. Damit verbunden kann dann beispielsweise die Ausbreitung von impliziten politischen Präferenzen und Problemrahmungen über Politikebenen hinweg und über die unmittelbare Instrumentenentwicklung und -implementierung hinaus analysiert werden.

Es geht in diesem Beitrag also darum, die Fragen zu beantworten: Welchen Mehrwert bringt eine sozialkonstruktivistische Perspektive auf Politikinstrumente in der europäischen Asylpolitik- und Asylverwaltungsforschung? Wie kann diese an bestehende Perspektiven auf Experten und Expertise in der Asylforschung anschließen?

Dieses Kapitel, das vor allem als theoretischer Beitrag zur Forschungsliteratur gedacht ist, möchte als Vorschlag gelten, sozialkonstruktivistische Perspektiven auf Politikinstrumente in der europäischen Asylpolitik und Asylverwaltung aufzunehmen. Damit lassen sich erstens die mit ihnen einhergehenden Festschreibungen von Vorstellungen über das Ordnungsverhältnis von Regierungsbzw. Regulierungsinstanzen und Gesellschaft offen legen, die inhärent im Spezialwissen von Experten zu spezifischen Politikinstrumenten sind. Zweitens kann das Sozialleben von Politikinstrumenten sichtbar gemacht werden, d.h. wie heterogene Akteure aus Wissenschaft, Politik, Verwaltung und Wirtschaft durch verschiedene miteinander vernetzte Tätigkeiten dazu beitragen, Instrumente zu artikulieren, zu stabilisieren, zu implementieren und zu verbreiten. Auf diesem Weg können dann auch drittens Nebeneffekte von Instrumenten, wie zum Beispiel die schleichende Erweiterung von deren Funktionsbestimmungen oder Neudeutungen, sichtbar und nachvollziehbar gemacht werden.

$\mathrm{Zu}$ diesem Zweck gliedert sich dieses Kapitel in vier Teile. Der folgende Abschnitt gibt einen empirischen Überblick über die Landschaft der europäischen Politikinstrumente, die den europäischen Asylverwaltungsraum konstituieren. In dem daran anschließenden Abschnitt zeigt sich, wie verschiedene Konzepte von Experten und Expertisenetzwerken und deren Anwendung in der Asylpolitikforschung bereits eine theoretische sowie eine empirische Grundlage geschaffen haben, die eine Verknüpfung mit und Erprobung von sozialkonstruktivistischer Politikinstrumentenforschung möglich und sinnvoll erscheinen lässt. Vor diesem Hintergrund folgt eine Skizzierung der zentralen analytischen Komponenten, die mit einer solchen Perspektive mobilisiert werden, die zur Illustration auf das Beispiel des Instruments EURODAC angewendet werden. Mit einer Erörterung 
des Mehrwerts als auch der Grenzen des hier unterbreiteten Vorschlags schließt dieses Kapitel.

\section{Bestandsaufnahme: Europäische Politikinstrumente und die politische Konstruktion des europäischen Asylverwaltungsraums}

Charakteristisch für die europäische Asylpolitik ist die fortwährende Richtigkeit der vor mehr als einem Jahrzehnt von Virginie Guiraudon aufgestellten Diagnose, dass sie »unvollständig und komplex« bleibt (Guiraudon 2003: 263). Auch nachdem seit gut fünfzehn Jahren an der Realisierung eines gemeinsamen europäischen Asylsystems (GEAS) gearbeitet wird, entfaltet es recht unterschiedlichen Einfluss auf die Mitgliedsstaaten (vgl. El-Enany/Thielemann 2011). Weder die Nationalstaaten noch die EU mit ihren Institutionen, wie der Kommission und dem europäischen Parlament, haben die eindeutige Oberhand in der Politikformulierung der europäischen Asylpolitik.

Historisch betrachtet haben die Nationalstaaten die dominierende Rolle in Fragen der europäischen Asylpolitik gespielt. Sie haben auch weiterhin eine starke Bedeutung. Sie nehmen in der Rechtssetzung Einfluss im Europäischen Rat und in der Umsetzung dadurch, dass sie Recht als Nationalstaaten ausführen. In relativ kurzer Zeit hat sich allerdings auch die EU als relevante Ebene für agenda-setting im Bereich von Migrations- und Asylpolitik etabliert. So haben sich die europäische asylpolitische Programmentwicklung und die dazugehörigen Politikinstrumente in Reaktion auf die Zunahme von Asylgesuchen zwar seit 1980 merklich weiterentwickelt. Dabei handelte es sich allerdings bis 1999 um zwischenstaatliche Zusammenarbeit. In dieser Zeit brachten die Mitgliedsstaaten und EU-Institutionen rechtliche Instrumente auf den Weg, die als geltendes nationales Recht die Verwaltungspraxis von nationalen Asylbehörden beeinflussten. Die Kooperation zwischen EU-Staaten begann sich nach zwei einschneidenden Neuerungen zu ändern. Die Verabschiedung des Dubliner Übereinkommens von 1990 führte die Regelung ein, die Zuständigkeit für Asylverfahren zwischen den Mitgliedsstaaten derart festzulegen, dass Asylbeantragungen im ersten EUEintrittsstaat des Asylsuchenden durchzuführen sind. Auf dem Ministertreffen 1992 einigten sich die Mitgliedsstaaten auf die Festlegung des Konzeptes von sogenannten »sicheren « Herkunftsstaaten und sicheren Transitstaaten. Letzteres hatte zur Konsequenz, dass Asylanträge von Asylsuchenden, die aus diesen Ländern stammten oder durchgereist waren, ein restriktiveres Verfahren zu erwarten 
hatten, einschließlich der Beweislastumkehr zu Lasten des Betroffenen. Das Augenmerk der zwischenstaatlichen Kooperation konzentrierte sich auf Polizeivollzug und Polizeibehörden sowie auf verstärkte Kontrollmechanismen (vgl. Guiraudon 2003: 268). Weitere Übereinkünfte wurden auf den europäischen Ministerkonferenzen 1994 und 1995 getroffen. Dazu zählte der Beschluss von Rücknahmeabkommen mit Transitstaaten, die als sicher eingestuft wurden. Diese Abkommen erleichterten die Abschiebungsmöglichkeiten durch nationale Ausländerbehörden in diese Länder (Trauner/Wolff 2014).

Obwohl die asymmetrische Verteilung von Asylsuchenden in den EUMitgliedstaaten in politischen Diskussionen thematisiert wurde und sich daran Forderungen nach gerechterer Lastenverteilung zwischen den Mitgliedstaaten anschlossen, kam es zu keinen substantiellen Veränderungen. Der Weg hin zu formaler Harmonisierung wurde mit dem Vertrag von Amsterdam von 1997 geebnet, der 1999 in Kraft trat. Dieser war das Ergebnis von Kompromissen, die aus den Aushandlungsprozessen zwischen Außenministerien (zuständig für die Verhandlung von Vertragsrevisionen, in diesem Fall die Integration des Schengener Abkommens in EU-Recht) und Innen- und Justizministerien (vormals zuständig für die Koordination des Schengenabkommens) hervorgingen (Guiraudon 2003: 270). Letztere, von Guiraudon »law and order officials« genannt, prägten und prägen die Diskursrahmung, wonach transnationale Migration in die EU als dauerhafte Sicherheitsbedrohung verstanden wurde (Guiraudon 2003: 272). Seit dem Amsterdamer Vertrag wurde der Einfluss der EU-Institutionen auf die Mitgliedsstaaten, insbesondere der Einfluss der Kommission, ausgebaut. Erst seit dem Gipfel von Tampere im Jahr 1998 strebt die EU eine rechtliche und administrative Harmonisierung nationalstaatlicher Asylpolitik an. Mit den Aktionsplänen von Tampere (1999-2004), Den Haag (2004-2009) und Stockholm (2009-2014) wurden gemeinsame Ziele etabliert, allerdings ohne dass diese mittlerweile umfassend erreicht wurden. Mittels EU-Richtlinien und Verordnungen wurden Ziele konkretisiert. Trotz harmonisierter rechtlicher Vorgaben lassen diese einiges an Vielfalt bei der Wahl der Mittel zu. Daher haben sich die Politiken der Mitgliedsstaaten vielfältig weiter ausgestaltet, sodass sich die Umsetzung der Rechtsvorgaben entsprechend massiv unterscheidet.

Die europäische post-Amsterdam Asylpolitik hat zunächst das sogenannte GEAS I und sodann das GEAS II hervorgebracht. Eine erste Phase war 2006 abgeschlossen (GEAS I) (Boswell/Geddes 2011: 151-152). Zwischen 2011 und 2013 kam es zur Novellierung der Rechtsgrundlagen (GEAS II). Deren Umsetzung in nationales Recht war bis Mitte 2015 vorgesehen. Die Sammlung an Richtlinien und Verordnungen, einschließlich der novellierten, umfasst nun die folgenden. Nach der Kosovo-Krise wurde 2001 ein Modus geschaffen, nachdem 
der Europäische Rat feststellen kann, ob es einen »Massenzustrom« an Vertriebenen und den Bedarf zu deren vorübergehenden Schutz gibt. In diesem Fall sollte dann ein Solidaritätsprinzip zwischen den Mitgliedsstaaten greifen (Massenzustrom-Richtlinie 2001/55/EG). Im Jahr 2008 wurden gemeinsame Regelungen zur Abschiebung sowie zur Anwendung von Zwangsmaßnahmen und Einreiseverboten festgelegt (Rückführungsrichtlinie). In einer weiteren Richtlinie sind nun gemeinsame Mindestnormen für die Anerkennung von Drittstaatsangehörigen oder Staatenlosen als Flüchtlinge oder subsidiär Schutzberechtigte und deren Rechte festlegt (Qualifikationsrichtlinie 2011/95/EU). Außerdem wurden Standards insbesondere für die Mindestaufnahmebedingungen und die Unterbringung (Aufnahme-Richtlinie 2013/33/EU) und für die Durchführung der Asylverfahren zur Zu- oder Aberkennung der Flüchtlingseigenschaft (Verfahrens-Richtlinie 2013/32/EU) stärker vereinheitlicht. Nach der sogenannten Dublin III-Verordnung ist festlegt, dass Drittstaatenangehörige meist in dem ersten Land Asyl beantragen müssen, in dem sie in die EU einreisen. Sie führt dazu, dass nationale Asylbehörden Antragsteller in das Land zurückschicken können, durch das sie eingereist sind. Die Verordnung bestimmt den für die Prüfung eines Asylantrags zuständigen Mitgliedstaat (Dublin-Verordnung 604/2013). Zum Zweck der effektiven Anwendung der Dublin-Verordnung wurden Regeln zum Abgleich von Fingerabdruckdaten definiert (EURODAC-Verordnung 603/2013).

Die Gewaltenteilung der europäischen Institutionen spielt in der Asylpolitik eine entscheidende Rolle - wenn auch nicht in ihrer klassischen Dreiteilung (vgl. Tömmel 2014: 88). In den im Migrationsbereich zur Regel gewordenen Mitentscheidungsverfahren teilen sich Kommission, europäischer Rat und das Parlament Aufgaben der Legislative. Die Kommission und die Mitgliedsstaaten teilen sich vor allem die Verantwortlichkeiten für Management und Implementation von Asylpolitik. Der europäische Gerichtshof entspricht der Judikative. Er spielte lange Zeit nur eine geringe Rolle, hat jedoch gemeinsam mit dem europäischen Gerichtshof für Menschenrechte in den letzten Jahren durch seine Präzedenzurteile an Einfluss in der Asylpolitik dazu gewonnen (vgl. Velutti 2014). Dazu zählen Urteile, die sich auf Griechenland und Italien bezogen, und die Regelung einführten, wonach eine Rücküberstellung von Asylsuchenden in Mitgliedsstaaten nicht erfolgen dürfe, wenn deren Asylsystem »systemische Mängel« aufweist (vgl. Pelzer 2012; Thym 2013). Mit diesen Instrumenten der Rechtsauslegung hat der europäische Gerichtshof Einfluss auf die europarechtskonforme Interpretation des bestehenden Rechts und wirkt damit auf die Umsetzung in nationales Recht durch die nationalen Parlamente und Gerichte. Damit werden auch die nationalen Asylverwaltungen beeinflusst, da Rechtsstandards für die Verwaltungspraxis gesetzt werden. 
Auch wenn seit dem Stockholmer Programm die Harmonisierung zu einem einheitlichen Asylsystem, dem GEAS, erklärtes Ziel ist (vgl. Boswell/Geddes 2011: 163) und perspektivisch zur stärkeren de facto Vereinheitlichung der Anerkennungsprozesse führen soll, bleibt die Umsetzung bislang eklatant unterschiedlich. Das liegt nicht zuletzt an den divergierenden Interessen der Mitgliedsstaaten. Diese intervenieren insbesondere über den Europäischen Rat, pochen auf ihre Souveränität und restringieren dadurch die Umsetzung der Richtlinien und Verordnungen.

Gemeinsam eingeführte Instrumente zur Umsetzung der Dublin-Verordnung, wie die Einrichtung von Datenbanken auf der Grundlage der EURODACVerordnung als Instrument zur Datenerfassung und zum Abgleich von Fingerabdrücken oder zum Grenzschutz, wie das Schengen-Information-System (SIS), dienen zwar der Vereinheitlichung der Datensätze und Verfahrensabläufe unter Einbeziehung der nationalen Grenzschutz-, Sicherheits- und Asylbehörden. Gleichzeitig wurden und werden diese Instrumente allerdings auch ganz unterschiedlich von Mitgliedsstaaten (nicht) genutzt. Insbesondere die ungleiche Verteilung von Asylsuchenden in den Mitgliedsstaaten, die in der DublinVerordnung rechtlich angelegt ist und die die EU-Außenstaaten stärker belastet, motiviert die Umgehung der Instrumente, die der Ersterfassung und Zuordnung von Asylsuchenden dienen.

Neben dem Bemühen, ein gemeinsames europäisches Asylsystem mit Rechtsinstrumenten (verbindliche Rechtsakte) zu etablieren, hat die Europäische Union eine Reihe von auf Freiwilligkeit basierenden Politikinstrumenten installiert. Sie verfolgen das Ziel, die Verwaltungspraxis von nationalen Behörden und Organisationen, die in die Implementierung der Asylpolitik involviert sind, zu koordinieren und zu harmonisieren. Im Jahr 2012 wurde ein einmaliges Umsiedlungsprogramm beschlossen, das mit finanziellen Anreizen Mitgliedsstaaten zur freiwilligen Aufnahme von Flüchtlingen bewegen soll. Das Grenzüberwachungssystem Eurosur, das durch die Grenzschutzagentur Frontex koordiniert wird, wurde 2013 zur Sicherung der Außengrenze und zur Rettung von Flüchtlingen in Seenot installiert. Im Jahr 2015 einigten sich die EU-Mitgliedsstaaten erstmalig auf einen Verteilungsschlüssel für die begrenzte Anzahl von 120.000 Flüchtlingen. Angesichts der drastisch gestiegenen Flüchtlingszahlen wird die Verteilungsfrage eine der zentralen Aushandlungsfragen bleiben. Darüber hinaus wurden Instrumente, die dem »capacity building« der nationalen Asylverwaltung dienen sollen, eingesetzt. Dazu wurde 2011 das Europäische Unterstützungsbüro für Asylfragen (EASO) eingerichtet, das Trainings von Verwaltungsbeamten durch Schulungen und Schulungsmaterialien zum Beispiel zu »Schutzgewäh- 
rung «, »Anhörungstechniken« oder »Beweiswürdigung« durchführt (Europäisches Unterstützungsbüro für Asylfragen 2014).

Angesichts der komplexen Dynamiken von europäischen Harmonisierungsbemühungen einerseits und nationalstaatlichen Einzelinteressen andererseits lassen sich vier Idealtypen von europäischen Politikinstrumenten identifizieren, die unterschiedlich Einfluss auf nationale Verwaltungen nehmen (vgl. Heidbreder 2011). Erstens zeigt sich, dass auf europäischer Ebene eingeführte Verordnungen sowie Richtlinien als umgesetztes nationales Recht in den nationalen Verwaltungen eine Rolle spielen (hierarchisch durchgesetzter administrativer Standard). Dazu zählen alle Richtlinien und Verordnungen, die im Zusammenhang des GEAS eingeführt wurden. Sanktionierungen von abweichender Praxis können durch die Rechtsauslegungsmöglichkeiten über den Europäischen Gerichtshof wie oben beschrieben erzielt werden. Auch die Dublin- und die EURODACVerordnungen sind Beispiele für den hierarchisch durchgesetzten administrativen Standard, die zwar durch die zentrale europäische Koordinierung geprägt sind, aber auf das Zusammenspiel mit nationalen Polizei- und Grenzschutzbehörden angewiesen sind. Zweitens kann durch die freiwillige Koordinierung zwischen EU-Mitgliedsstaaten die Verwaltungspraxis Beeinflussung erfahren (nicht-hierarchische freiwillige Koordinierung). Beispiel hierfür ist das Umsiedlungsprogramm für Flüchtlinge mit finanziellen Anreizen zur Umsiedlung. Drittens lassen sich Instrumente identifizieren, die sich durch die Implementierung auf europäischer Ebene indirekt auf nationale Verwaltungen auswirken (hierarchische Verwaltungsanleitung). Charakteristisch ist hier, dass es sich um impliziten Einfluss auf nationale Verwaltungen handelt, indem ein spezifisches, einheitliches Modell zum Zweck des »capacity building « hierarchisch vermittelt wird. Hier sind die Services des EASO zur Schulung von Verwaltungsbeamten zu vereinheitlichten Anhörungsmethoden von Asylsuchenden oder zur Bereitstellung von vereinheitlichten Herkunftslandinformationen ein Beispiel. Ein vierter Idealtyp nach Heidbreder (2011: 721) ist eine Form der indirekten Kontrolle, ob die Durchführung von Verwaltungspraxis effektiv und funktionstüchtig ist (nichthierarchische Konformitätskontrolle). Dies kann durch Instrumente der Berichterstattungsverfahren und durch statistische Datenerhebung erfolgen. In dieser Kategorie ließe sich exemplarisch das von der EU-Kommission eingesetzte European Migration Network, beauftragt mit der europäischen Migrationsstatistik, einordnen. Auf das genannte empirische Beispiel des letzten Typs wird ausführlicher im folgenden Abschnitt eingegangen. 


\section{Neuere Forschungsperspektiven in der europäischen Asylforschung: Konzepte von Experten und Expertise}

Politikverantwortliche und Verwaltungsbeamte in der europäischen Asylpolitik sind zunehmend von Expertise und von spezialisierten Wissensformen abhängig, über die spezialisierte Expertengruppen zum Beispiel aus der humanitären Hilfe, aus arbeitsmarktregulierenden oder sicherheitstechnischen Bereichen verfügen. Akademisches Wissen wird mit der generell wachsenden Bedeutung von Paradigmen der evidenzbasierten Politikentscheidung auch in der Asylpolitik von politischen und administrativen Entscheidungsträgern nachgefragt. Wissenschaftliche Expertise kann daher dazu beitragen, die bestehenden politischen Präferenzen zu legitimieren und zu signalisieren, dass die Kapazität vorhanden ist, fundierte und evidenzbasierte Entscheidungen zu treffen (vgl. Boswell 2012). Aber auch Nichtregierungsorganisationen beraten die Kommission, wie Virginie Guiraudon anschaulich für die europäische Anti-Diskriminierungsregulierung gezeigt hat. Anti-Diskriminierung setzte sich in Kopplung mit der erfolgversprechenden diskursiven Rahmung von »sozialer Exklusion« in der Migrationsdebatte nicht zuletzt durch die erfolgreiche Beratung durch die in Brüssel ansässige Nichtregierungsgruppe Migration Policy Group (MPG) durch (vgl. Guiraudon 2003). Spätestens seitdem neue Technologien im Grenzkontrollmanagement Einsatz finden, ist auch die Sicherheits(informations)technik eine nachgefragte Expertise (vgl. Aas 2006; Tholen 2010). Soziologische Beiträge, insbesondere der politischen Soziologie zur Europaforschung (Favell/Guiraudon 2011) haben erste interessante Beiträge geliefert, um Expertise, verschiedene Wissensformen und professionelle Netzwerke gezielt zu berücksichtigen und ihren Einfluss auf die europäische Asylpolitik und Asylverwaltung(en) zu erfassen. Einige Autoren (vgl. Boswell 2012; Geddes 2015) haben sich im engeren Sinne darauf spezialisiert, die Wissensproduktion (vor allem die wissenschaftliche) und die Rolle von Expertise (von Wissenschaftlern) in der Beziehung von Forschung und Politik im europäischen migrationspolitischen Kontext näher unter die Lupe zu nehmen. Der Fokus von Forschung, die sich mit den daraus resultierenden Fragen für die Asylpolitik beschäftigt, liegt vor allem auf dem Verhältnis zwischen Wissenschaft und Politik und untersucht die Dynamiken der Verwissenschaftlichung der Politik und der Politisierung der Wissenschaft. In einer Untersuchung zur Interaktion zwischen EU-Kommission und dem European Migration Network, welches einen maßgeblichen Beitrag zur Migrationsstatistik leistet, treten dabei verschiedene Wechselbeziehungen zu Tage (vgl. Geddes 2015). Erstens kommt zum Vorschein, dass wissenschaftliche Expertise instrumentalisiert wird, um die 
Wahl von Politiken zu begründen (»evidence-based policymaking«). Allerdings zeigt sich zweitens auch, dass getroffene Politikentscheidungen langfristig den Kontext prägen, in dem Wissen entwickelt wird (»policy-based evidencemaking«). Schließlich erweist sich aber auch die Informationsbereitstellung und Wissensproduktion als Verstetigung der Legitimation für institutionelle Rollen (z.B. der Kommission) (»policy-based institution-building«). Während sich Geddes (2015) Untersuchung auf die Praktiken der europäischen Migrationsund Asylstatistik bezieht, beschreibt Christina Boswell (2012) in ihrer komparativen Analyse der britischen, deutschen und europäischen Asylpolitik die politische Verwendung von Expertenwissen. Hier gelten insbesondere Wissenschaftler als Experten. Dabei fokussiert sie neben der Europäischen Kommission einerseits auf das deutsche Bundesamt für Migration und Flüchtlinge (BAMF) und die hausinterne Einrichtung einer Forschungsgruppe 2004 und andererseits auf das British Home Office und die Erlassung eines Forschungsprogrammes zu Einwanderung und Asyl im Jahr 2000, angesiedelt an einem neu geschaffenen Immigration Research and Statistics Service (IRSS). Inspiriert durch neoinstitutionalistische Organisationstheorie interessiert sie sich für »political organizations« (in Abgrenzung von »action organizations«) (vgl. Brunsson 2002). In diesen Fällen sind es Regierungsorganisationen, die anhand ihrer formalen Strukturen und Entscheidungen und weniger an den sozialen Auswirkungen ihrer Entscheidungen gemessen werden (vgl. Boswell 2012: 13). Die Legitimität der Asylbehörden wird hier auf der Grundlage konstruiert, dass sie einen Nachweis erbringen können, dass ihre Entscheidungen auf der Grundlage von solidem Wissen geschehen. Dieses wird vor allem dann relevant, wenn es sich in Folge von kontroversen politischen Debatten um umstrittene politische Gegenstände handelt und mit dem Nachweis der wissenschaftlichen Evidenz der Erfolg und die Richtigkeit von ergriffenen Maßnahmen und die Angemessenheit zur Zielerreichung untermauert werden sollen. Nach einer Unterscheidung von Expertenwissen nach Funktion und Nutzung im Verhältnis zu bestimmten Arten von Politikdebatten (vgl. Boswell 2012) ließen sich die beschriebenen Fälle von Boswell und Geddes der Funktion der Erbringung von Evidenz zur Verifikation von Politikmaßnahmen zuordnen. Die beiden vorgestellten Studien beziehen sich insbesondere auf wissenschaftliche Expertise und die Rolle von Wissenschaftlern.

Zur Erfassung von Expertisen, die über einen Fokus auf rein wissenschaftliches Expertenwissen hinaus gehen, haben auch andere Konzepte in der europäischen Asylforschung Anwendung gefunden: einerseits jene, die sich für Experten als Akteure, die kausal Asylpolitik beeinflussen, interessieren; andererseits solche, die die Praktiken in Verbindung mit Expertise betrachten, die die episte- 
mischen Rahmenbedingungen von Asylpolitik prägen (vgl. Bueger 2014). Im Folgenden sollen Beispiele zu beiden Kategorien vorgestellt werden.

$\mathrm{Zu}$ ersteren zählt das Konzept der »epistemic communities«. ${ }^{3} »$ Epistemic communities« sind Netzwerke von Professionellen mit anerkannter Expertise und Kompetenz in einem bestimmten Bereich und beziehen sich auf bestimmtes politikrelevantes Wissen innerhalb eines Sektors oder mit Bezug auf einen bestimmten Politikgegenstand (vgl. Haas 1992: 3). Was diese Experten verbindet und gleichzeitig von anderen Akteurstypen abgrenzt, sind erstens ein geteiltes Set an Wertvorstellungen, zweitens gemeinsame Überzeugungen von Kausalzusammenhängen, die von der eigenen Analyse der Praxis abgeleitet werden und drittens geteilte Vorstellungen von Validität, welche als intern definierte Kriterien verstanden werden, die dazu dienen, Wissen zu bewerten und zu validieren (ebd.). Darüber hinaus beziehen sie sich auf ein gemeinsames Politikunternehmen. Damit sind gemeinsame Praktiken gemeint, die sich Problemstellungen widmen, die im Kern der Kompetenz der Experten liegen (ebd.). In diesem Konzept sind Experten abgrenzbar von anderen Akteursgruppen wie politischen Entscheidungsträgern oder Verwaltungsbeamten. Diese wiederum können durch die Beratung von Experten lernen. Das Wissen, das durch »epistemic communities« bereitgestellt wird, kann sich durch Diffusionsprozesse innerhalb von Verwaltungen, Regimen und Organisationen verbreiten und gegebenenfalls institutionalisieren. Dabei können sich geteilte Interpretationen (vgl. Adler/Haas 1992: 371), Werte und Praktiken (ebd.: 372), aber auch intellektuelle Innovationen (ebd.: 373) verbreiten. Wenn diese politische Entscheidungsträger erreichen und von diesen aufgegriffen werden, können sie Standardsetzungsprozesse und die Entwicklung von Politiken beeinflussen (ebd.: 379). Im Zentrum steht die Interaktion zwischen Experten und politischen Entscheidungsträgern.

Boswell (2005) hat diese Art von epistemischen Gemeinschaften im Feld der Migrationspolitik identifiziert. Sie verweist auf Forschungseinrichtungen und Universitäten, die entweder beratend für Regierungseinrichtungen oder eigenständig ihre Forschungsergebnisse verbreiten. Aber auch wissenschaftliche Mitarbeiter, Juristen und Politikberater in NGOs und anderen Lobbygruppen zählen dazu. Boswell (2005: 11) weist darauf hin, dass akademische Expertise gerade in Hinsicht auf die instrumentelle und technische Funktion von Wissen, seine An-

3 Diverse Konzepte zur Analyse von Expertennetzwerken, die spezielle Politiken unterstützen, wurden in den vergangenen zwei Dekaden entwickelt. Dazu gehören »advocacy coalitions « (Sabatier/Jenkins-Smith 1993), »epistemic communities« (Haas 1992), »discourse coalitions« (Hajer 2006), »transnational communities« (Djelic/ Quack 2010). 
wendbarkeit und spezifische Passung zu Anwendungsproblemen in diesem Zusammenhang nur begrenzt relevant ist. Die Vorfestlegung von politischen Ausrichtungen definiert zusätzlich auch die Anforderungen, die an nutzbares Expertenwissen angelegt werden. Doch selbst anwendungsorientierte Forschung muss an das Wissen nicht anschlussfähig sein, welches in der politischen und Verwaltungspraxis benötigt wird. Aus diesem Grund zeichnet sich das Wissen von Experten beispielsweise in Nichtregierungsorganisationen und von Sozialpartnern dadurch aus, dass sie das Erfahrungswissen und das gemeinsame Grundverständnis von Anwendungskontexten haben.

Bei der Nutzung des Konzeptes der epistemischen Gemeinschaften wird insbesondere auf ein Verständnis von Expertenwissen aufgebaut, welches als solches sozial anerkannt wird und häufig mit akademischer Expertise oder technischem Wissen in Verbindung gebracht wird (vgl. Cross 2013a). Es hat auch in an die Asylthematik angrenzenden Themenfeldern analytische Anwendung gefunden, so zum Beispiel im grenzüberschreitenden Kriminalitätsmanagement in Südosteuropa (vgl. Woodward 2004) oder in der militärischen Dimension der europäischen Sicherheitspolitik (vgl. Cross 2013b). Für die Spezifizierung des Konzeptes für die Nutzung im Zusammenhang mit einzelnen Politikinstrumenten sollte im Blick bleiben, dass es durch seinen Rückbezug auf akademische und technische Expertiseformen für bestimmte Instrumente relevanter sein kann als für andere: »the more dependent an issue area becomes on technical information, the greater the likelihood that epistemic communities gain in influence« (Haas 1992: 592). Das Konzept findet vor allem Anwendung, um die kausale Wirkung von Wissen auf Politik zu untersuchen. Wissen selbst wird als gegeben vorausgesetzt. Aus der kritischen Rezeption des Konzepts haben sich auch Deutungen entwickelt, die die soziale und politische Komponente von Wissensproduktion rekonstruieren (vgl. Antoniades 2003).

Die zweite Kategorie von Konzepten bezieht sich auf die epistemische Praxis, also die Praktiken von unterschiedlichen Akteuren, die Wissensdeutungen und Interpretationen prägen. Bigo (2014) und Huysmans (2006) haben sich im Bereich der Sicherheitsstudien, und aus diesem Blickwinkel auch der Asylpolitik und -verwaltung, der Frage gewidmet, wie sich die Bedeutungen von Sicherheit quer zu Akteursgruppen konstituieren. In dieser Perspektive unterscheiden sich wissenschaftliche Experten, politische Entscheidungsträger, Grenzschutz, Polizisten und Verwaltungsbeamte vor allem durch die unterschiedlichen wissensbezogen Praktiken, nicht zu allererst durch die Wissensform (vgl. Bueger 2014). Didier Bigo (2006) analysiert unter dem theoretischen und methodischen Einfluss von Bourdieu das transnationale Feld der professionellen Sicherheitsspezialisten. Er benutzt Bourdieus Feldkonzept und Habituskonzept, um die Gewohn- 
heiten und automatischen Reflexe zu analysieren, die professionelle Spezialisten während ihrer Sozialisation in verschiedenen Rollen und Positionen entwickeln. Bonelli und Bigo (2005) benutzen eine Methode des »mappings«, um die Hypothese eines europäischen Feldes der Sicherheit quer zu Instanzen der Rechtsdurchsetzung, Geheimdiensten und öffentlichen Verwaltungen zu rekonstruieren. Dabei geht es um die Akteure (und deren Arbeitspraktiken), die dafür sorgen, dass es zur Durchsetzung eines gewissen »common sense« darüber kommt, was Sicherheit ist und was nicht (Bigo 2006). Dieser wird dann akzeptiert oder verstärkt durch professionelle Akteure in den Bereichen von Politik und Medien. Das Verbindende in einem solchen Feld sind die Vorstellungen von Sicherheit. Dabei werden analog zu Bourdieus Feldkonzept die verschiedenen Formen von Kapital aufgegriffen. Interessant sind dann aus dieser Perspektive die Strukturierung des Feldes durch EU-Richtlinien und Datenbanksysteme wie EURODAC und Prüm, die den Austausch von biometrischen Daten von Asylsuchenden bzw. kriminellen Tatverdächtigen in der EU regeln. Der Vertrag von Prüm, der zunächst als Vertrag zwischen sieben Staaten 2005 unterzeichnet wurde, regelt den Datenaustausch zwischen den beteiligten Staaten zu Angelegenheiten, die den Kampf gegen organisierte Kriminalität, Terrorismus und sogenannte illegale Migration betreffen. Asylpolitik und -verwaltung wurden hier hinsichtlich der Frage in den Blick genommen, inwiefern die Maßnahmen im Kampf gegen Terrorismus sich auch in anderen Politikbereichen auswirken. Die Asylverwaltung wird in diesen Studien tangiert, allerdings eben nur, sofern sie mit dem Fokus auf Sicherheitsfragen und in Bezug auf sogenannte »illegale Migration« betrachtet wird, so zum Beispiel die Folgen auf die Möglichkeiten bzw. Begrenzungen, Asyl faktisch zu erhalten. Mit diesem Ansatz lassen sich vor allem Prozesse der Institutionalisierung von Deutungshoheiten - wie zum Beispiel die Illegalisierung und Kriminalisierung von Migration - untersuchen. Augenmerk liegt dabei auf professionsspezifischen epistemischen Praktiken - in diesem Fall des sicherheitspolitischen Feldes.

Während die vorgestellten Ansätze zu Expertise und Experten der Entwicklung der politischen Europäischen Soziologie generell entsprechen, in der sich neo-institutionalistische, sozialkonstruktivistische und eben wissenssoziologische Einflüsse bemerkbar machen (vgl. Favel/Guiraudon 2011), haben sich andere neuere Trends in der soziologischen Europaforschung mit Bezug auf die Asyl- und Migrationspolitik bislang kaum niedergeschlagen. ${ }^{4}$ Dazu zählt ein sys-

4 Eine Ausnahme stellt der Beitrag von Trauner und Wolff (2014) dar. In diesem einleitenden Beitrag zu einem Special Issue bieten sie einen Analyserahmen für die Untersuchung von Verhandlung von Politikinstrumenten in der EU-Migrationspolitik an. In 
tematischer sozialkonstruktivistischer soziologischer Blick auf europäische Politikinstrumente als auch darauf, wie diese selektive Repräsentationen und Bedeutungen transportieren und sich direkt und indirekt auf die Asylverwaltung(en) in Europa und in den Mitgliedsstaaten auswirken. Dabei kann gerade eine Anknüpfung an die bereits existierenden und beschriebenen Perspektiven auf Expertise und Experten eine fruchtbare Verknüpfung sein, die die Etablierung von bestimmten Wissensformen und -praktiken entlang von Politikinstrumenten und deren Prägung von Politik- und Verwaltungspraxis nachvollziehbar macht.

\section{Experten und Expertise in europäischen Asylpolitikinstrumenten}

Um zu erörtern, wie eine sozialkonstruktivistische Perspektive auf Politikinstrumente für den europäischen Asylverwaltungsraum nutzbar gemacht werden kann, werde ich im Folgenden zunächst konzeptionelle Komponenten einführen, um diese dann an einem europäischen asylpolitischen Beispiel zu veranschaulichen. Zur näheren Klärung, in welcher Weise Politikinstrumente ihren Einfluss entfalten können und in welcher Weise Experten und Expertise dabei eine Rolle spielen, hilft zunächst Lascoumes and Le Galès (2007: 4, Herv.i.O.) Definition von Politikinstrumenten:

$»$ A public policy instrument constitutes a device that is both technical and social, that organizes specific social relations between the state and those it is addressed to, according to the representations and meanings it carries. It is a particular type of institution, a technical device with the generic purpose of carrying a concrete concept of the politics/society relationship and sustained by a concept of regulation. «

Zur Einordnung dieser Definition möchte ich drei Erläuterungen vornehmen. Erstens entwickeln Lascoumes und Le Galès ihr Verständnis von »technisch« einerseits in dem Sinne, dass Politikinstrumente mit der Bestimmung entwickelt werden, technisch-funktionale Lösungen zu spezifischen Problemen zu liefern (ebd.: 2, 4). Andererseits bestehen Instrumente selbst aus technischen Kompo-

diesem wird nach Instrumentenetablierung und Nutzung unterschieden und sie fokussieren sich auf das Instrumentendesign. Sie stellen zwei Formen der Anfechtung von Instrumenten zur Diskussion: Zum einen kann durch Politiktransfer und Neuinterpretation ein Instrument in Frage gestellt werden, zum anderen können Instrumente im Zuge ihrer Instrumentalisierung verwässern. 
nenten wie Kalkulationen, Berechnungen, standardisierten Prozederen, die darauf abzielen, die Kalkulierbarkeit und Vorhersehbarkeit von Politik zu erhöhen (ebd.: 7). Zweitens bestehen Instrumente aber auch aus »sozialen« Komponenten. Sie transportieren Repräsentationen von Ordnungsverhältnissen zwischen Regierenden und Regierten oder Verwaltenden und Verwalteten (ebd.: 11). Drittens ergeben sich für die Autoren »konkrete Konzepte von Politik-GesellschaftsBeziehungen « in Instrumenten, da diese aus impliziten politischen Theoretisierungen hervorgehen. Instrumente manifestieren sich dann, und hier beziehen sich die Autoren auf den französischen Wissenschaftstheoretiker Gaston Bachelard, als »Konkretisierung einer Theorie«. Instrumente konstituieren »a condensed and finalized form of knowledge about social control and ways of exercising it« (ebd.: 11). Lascoumes und Le Galès entwickeln dann in Folge eine Typologie von Instrumenten, bei der sie idealtypische Repräsentationen von Ordnungsverhältnissen bestimmten Instrumenten zuordnen (ebd.: 12). ${ }^{5}$ Um die Relevanz von Wissensproduktionsprozessen für Politikinstrumente deutlicher zu offenbaren, hat $\mathrm{Vo} ß$ darauf hingewiesen, dass der Entstehungsprozess von Instrumenten selbst mehr Beachtung verdient, bei dem Wissen und damit einhergehende Repräsentationen von politischen Ordnungsentwürfen von heterogenen Akteuren in die Konzeption und Modellierung von Instrumenten einfließen. Gleichermaßen relevant ist, wie sich Wissen und Repräsentationen auf deren (Neu)Interpetation während der Implementierung von Instrumenten auswirken (vgl. Voß 2007a: 177; Voß 2007b).

Zwei Aspekte möchte ich hier nun im Besonderen herausstellen. Zum einen lenkt eine solche sozialkonstruktivistische Perspektive auf Instrumente das Augenmerk auf die Ko-Produktion von politischen Ordnungsentwürfen in Modell und Wirklichkeit. Während der Instrumentenentwicklung bringen involvierte Akteure selektives Wissen, zum Beispiel durch die Einbringung neuer Forschungserkenntnisse oder neuer Technik, in die Modellierung von Instrumenten ein. Dieses wird im Zuge von fachlichen und politischen Aushandlungsprozessen zu Modellen des Instrumentes konsolidiert, womit ausgewählte Vorstellungen von Politik und Gesellschaftsbeziehungen einhergehen. In der politischen Umsetzung kommt es zur Etablierung neuer politischer Koalitionen und zu weiteren Übersetzungs- und Konfigurierungsprozessen des Modells in die Praxis.

5 Sie unterscheiden zwischen verschiedenen Instrumententypen (zum Beispiel Legislative und Regulierende, Ökonomische und Fiskalische oder De Facto und De Jure Standards). Diese ordnen die Autoren verschiedenen politisch-theoretisierten Ordnungsentwürfen zu, die sich jeweils aus verschiedenen Legitimationsmustern herleiten und damit unterschiedliche Wertesysteme favorisieren. 
Dabei interpretieren Akteure, die in die Implementation der Instrumente involviert sind, diese durchaus weiter und verändern diese. Sie rekonfigurieren beispielsweise die (technisch) möglichen Einsatzmöglichkeiten. Die eingeschriebenen Ordnungsentwürfe und die damit einhergehenden Wertvorstellungen bleiben oft erhalten oder bilden die Struktur an der sich Akteure orientieren, um gezielt auch in Abgrenzung - nachzujustieren.

Zum anderen bietet es sich an, die »Zuliefererseite« von Politikinstrumenten und das »soziale Leben« hinter ihnen in den Blick zu nehmen (vgl. Voß 2007a: 177-179; Voß/Simons 2014), das heißt also die Akteursgruppen, die Instrumente gestalten. Der Fokus richtet sich dann auf die sozialen Gruppen, die zur historischen Entwicklung beitragen, also an der Entstehung, Verbreitung und Veränderung von Politikinstrumenten in einer - nicht notwendigerweise als solche explizit gemachten - arbeitsteiligen Weise mitwirken. Heterogene Akteure, zum Beispiel aus Wissenschaft, Wirtschaft, Politik und Verwaltung, sind vereint durch das gemeinsame Interesse an einem Politikinstrument. Sie tragen durch vielfältige Arbeitspraktiken zur Entwicklung und Verbreitung des Instrumentes bei. Dabei verbindet sie nicht zwingend ein gemeinsames Verständnis des Instrumentes. Ein noch recht junges Konzept zur Erfassung dieser Akteursgruppe ist das der »instrument constituencies «, was bislang empirisch Anwendung findet für umweltpolitische Instrumente wie dem Emissionshandel (vgl. Voß/Simons 2014) und dem Biodiversitätszertifikatehandel (vgl. Mann/Simons 2015), Bürgerbeteiligungsverfahren (vgl. Amelung/Grabner im Erscheinen) sowie sozialpolitische Instrumente wie Systeme der Sozial- und privaten Rentensozialversicherung (vgl. Béland/Howlett 2015). Ein sorgfältiger Nachweis, ob sich »instrument constituencies« empirisch in Instrumenten der europäischen Asylverwaltung finden lassen, steht aus. Anhand einer folgenden illustrativen Anwendung der beschriebenen analytischen Perspektive auf das Beispiel EURODAC lässt sich jedoch vermuten, dass dies der Fall sein kann. Ebenso zeigt sich an dem Beispiel, wie sich Expertise, insbesondere manifestiert in biometrischen Technologien, und heterogene spezialisierte Akteure aus Wirtschaft, Wissenschaft, Politik und Verwaltung die Etablierung von EURODAC erwirken und dessen Ausgestaltung prägen.

Das Instrument EURODAC (Akronym für European dactyloscopic system) ist eine Maßnahme, die in Verbindung mit dem Schengen- und Dublinregime in einer europäischen Datenbank die Fingerabdrücke von Asylsuchenden bzw. irregulären Einwanderern erfasst und vergleicht. Es organisiert und trägt zur Bestimmung bei, welcher EU-Mitgliedsstaat verantwortlich für die Bearbeitung eines Asylantrages ist. Durch ein standardisiertes Prozedere wird reguliert, dass Fingerabdruckdaten von nationalen Autoritäten an die Zentraleinheit, die von der 
Kommission geführt wird, weitergeleitet werden, um zentral die Daten zusammenzuführen und mit vorherigen Einträgen zu vergleichen oder - je nach Datenkategorie - durch die Speicherung auf Vorrat für spätere Abgleiche zur Verfügung zu haben. Ein »Treffer« ergibt sich nach der Logik des Systems dann, wenn der Datensatz über einen bestimmten Fingerabdruck bereits existiert. Daraus wird dann geschlossen, dass ein Drittstaatenangehöriger mehrfach erfasst wurde und dessen Daten durch einen Mitgliedsstaat bereits übermittelt wurden.

Betrachtet man den Entstehungskontext von EURODAC, sind es vor allem technische Experten aus der Sicherheitstechnik und der biometrischen Industrie, die als Akteursgruppe eine zentrale Rolle spielten. Darüber hinaus ist es später die Expertise der Polizei-Daktyloskopen und Ministerialbürokraten aus den nationalen Innenministerien, die die weitere Ausgestaltung von EURODAC prägen. Irma van der Ploeg bezeichnete die Nutzung von Biometrie als Transformation von Körpermerkmalen in lesbare digitale Codes als »das nächste große Ding in der Informationstechnologie« (1999: 295). Biometrie wurde von der MIT Technology Review vom Januar 2000 als eine der »Top Ten« innovativen Technologien bezeichnet, die die Welt verändern werden. Nicht zuletzt aus kommerziellem Interesse bot die biometrische Industrie technische Lösungen für Probleme an, mit denen sich in erster Linie Polizei und Grenzschutz praktisch auseinandersetzen müssen (Aus 2003, 2006). Firmen, die »Automated Fingerprint Identification System« (AFIS) und unterstützende Hilfsmittel anbieten, warben damit, dass diese Technik zur Kontrolle oder Bekämpfung von Migration, Kriminalität und Terrorismus dienen kann und verfolgten damit das Interesse, ihr Produkt möglichst vielseitig nutzbar für verschiedene Anwendungsfelder anzubieten und zu verkaufen (Aus 2006: 5). Biometrische Identifikationstechnologien wie AFIS wurden mit dem Versprechen von optimierter Effizienz und Effektivität von polizeilichen Kontrollen assoziiert. Mit dieser Technologie wurden Schnelligkeit und wissenschaftliche Genauigkeit in der Identifizierung und im Datenabgleich sowie vielfältige Suchmöglichkeiten in großen Datensätzen verknüpft, was den Anreiz für Polizei und Grenzschutz erhöhte (ebd.).

In der Anbahnung von EURODAC standen solche Technologien also für den Zweck der Vereinfachung und zunehmenden Wirksamkeit der Migrationskontrolle. Gleichzeitig gingen mit ihnen aber auch von Beginn an wenig explizite Gesellschaftskonzeptionierungen einher. Implizit blieb dabei, wie sie Festschreibungen des individuellen Körpers manifestieren, die diesen mit Identität und Identifizierungsmechanismen gleichsetzen. Verankert wurde diese Festschreibung durch die Verknüpfung der Entnahme von Fingerabdrücken, deren Sammlung und Aufbewahrung in einer zentralen Datenbank und der Kopplung mit 
biometrischem Erkennungsequipment sowie der Möglichkeit der automatisierten Suche (vgl. Ploeg 1999: 301).

Die politischen Aushandlungsprozesse über die Ausgestaltung eines europaweiten Datenbanksystems für Fingerabdrücke begannen 1992 auf EU-Ebene. Die technisch mögliche Ausweitung der Datenbank für andere Zwecke als die ursprünglich definierten weckte schnell das Interesse von rechten als auch linken Politikern gleichermaßen (vgl. Kasparek/Tsianos 2015). Insbesondere die Frage, wessen biometrische Daten gesammelt werden sollten, offenbarte die unterschiedliche Auslegung der Technologie unter den Mitgliedsstaaten. Während einige Staaten insbesondere den Schutz von Flüchtlingen betonten und damit die Gruppe von potentiellen Flüchtlingen im Sinne der Genfer UN-Flüchtlingskonvention als Zielgruppe sahen, standen demgegenüber jene Staaten, die dem Instrument den Zweck der Kontrolle von illegaler Migration im Zusammenhang mit Menschenhandel und organisierter Kriminalität einschreiben wollten. Damit vergrößerte sich die Zielgruppe um jene Personen, die auf irregulärem Weg europäische (Außen)Grenzen überschreiten oder sich illegal in einem EUMitgliedsstaat aufhalten (vgl. Aus 2006: 7). Mit der erweiterten Kategorie von Personen, die mittels EURODAC erfasst werden, wurde zunächst die Verbindung zwischen Asylsuchenden und Illegalität bzw. illegaler Migration forciert. Menschenrechtsorganisationen sahen von Beginn an in der routinemäßigen Personenkontrolle von Asylsuchenden auf der Grundlage von biometrischen Merkmalen eine Verletzung der Menschenrechte und insbesondere des Rechts auf Privatsphäre (vgl. Ploeg 1999: 300).

Die erste Regulierung von Dezember 2000 kreierte schließlich die Rechtsbasis für die umfassende und systematische Erfassung von biometrischen Daten. Es bedurfte einer weiteren Regulierung im Jahre 2002, die eine technische und administrative Umsetzung möglich machte. Daten wurden dann ab Januar 2003 erfasst. Neben den Juristen aus Innenministerien, die an der Entwicklung der Verordnung beteiligt waren, involvierte das Instrument naheliegenderweise Grenzschutz, Polizei und Ausländer- und Asylbehörden.

In der Implementierung von EURODAC stellten vor allem einzelne Mitgliedsstaaten wie Italien oder Griechenland, in denen besonders viele potentielle Asylsuchende über EU-Außengrenzen einreisten, durch die faktische Nichtanwendung der Verordnung sowohl das EURODAC-System als auch die Legitimation des Dublin-Systems in Frage. Dabei ging es vor allem um das den beiden Verordnungen inhärente politische Konzept der Lastenverteilung zwischen den EU-Mitgliedsstaaten, welches auf diesem Weg ausgehebelt wurde. Durch die in einzelnen Ländern praktizierte Nichterfassung von potentiellen Asylsuchenden und damit das Ausbleiben, die Verantwortlichkeit für die Durchführung des 
Asylverfahrens festzulegen, fand EURODAC sehr unterschiedlich Anwendung (vgl. Kasparek/Tsianos 2015: 14).

Insbesondere zu dem Zweck der Terror- und Kriminalitätsbekämpfung kam es auf Initiative der Europäischen Kommission, die im Feld der Innen- und Justizpolitik in der Regel durch die Mitgliedsstaaten beauftragt wird, seit dem Jahr $2008 \mathrm{zu}$ Versuchen, die EURODAC-Verordnung zu ändern. Diese stießen jedoch auf vielfältigen Widerstand. Im Jahr 2013 schließlich kam es zu einer Neufassung der EURODAC-Verordnung (Mendos Kuşkonmaz 2013: 99). In diesem Zusammenhang wurde das Zugriffsrecht auf Strafverfolgungsbehörden und Europol erweitert. Gleichzeitig wurden nach vorhergegangenen Beanstandungen der europäischen Datenschutzbehörde Datenschutzregelungen ergänzt und spezifiziert. Diese beinhalteten eine Informationspflicht gegenüber den von der Datenverarbeitung betroffenen Personen sowie geänderte Aufbewahrungszeiten für Daten (vgl. Kasparek/Tsianos 2015: 13-14). Eine weitere Zugriffserweiterung des Instrumentes hin zu einer Ausdehnung der zu erfassenden Daten steht mit einem Vorschlag der Europäischen Kommission zur Novelle der EURODACVerordnung aktuell zur Diskussion. Europäischer Rat, Parlament und Kommission verhandeln seit 2016 darüber, auch persönliche Daten zur Gesichtserkennung und Reisedokumente zu erfassen, sowie die Altersgrenze der biometrischen Registrierung von 14 auf 6 Jahre zu senken (Europäische Kommission 2016).

Durch die Ausdehnung von Zugriffsmöglichkeiten auf EURODAC hin zur routinemäßigen Kriminalitätskontrolle von Personen, die durch EURODAC erfasst werden, haben sich schrittweise politische Repräsentationen ergeben, die eine Kriminalisierung der Migrationskontrollen (vgl. Aas 2011) und eine Digitalisierung der Außengrenzen, aber auch der Grenzkonflikte (vgl. Tsianos 2015) beschreiben. Der komplexe Apparatus aus technischen Datenbanksystemen und administrativer Bearbeitung ist dabei sowohl anfällig für technische Fehler (z.B. in Einzelfällen die Feststellung von falschen Treffern, die nicht weiter verifiziert werden) als auch für menschliche Fehler (z.B. die Ergänzung falscher Angaben zur Person) (vgl. Töpfer 2015).

Der EURODAC-Fall zeigt erstens, wie unterschiedliche Akteure an der Modellierung und weiteren Konfigurierung des Instrumentes beteiligt sind und zweitens, wie diese im Zusammenspiel miteinander bestimmte Einschreibungen vornehmen. Dabei involviert EURODAC als kontinentale Datenbank mittlerweile über 32 Staaten und alle Ebenen des europäischen Mehrebenensystems. Das schließt Erstaufnahmestellen und Kontrollposten der Grenzpolizeien, sowie Einwanderungsbehörden und Akteure der zentralen Datenbank in Straßburg und deren Verwalter in Talinn ein. Politische und gesellschaftliche Ordnungsentwürfe werden dabei implizit durch die Kopplung mit dem Instrument manifestiert, 
wenngleich sie auch auf Abwehr stoßen können. Jedoch sind die zugrundeliegenden politischen Repräsentationen selbst kaum explizit Gegenstand des politischen Auseinandersetzungsprozesses. Das liegt nicht zuletzt daran, dass sie durch sozio-technische Verkopplungen in gewisser Weise verschleiert werden.

\section{Schlussfolgerung: Möglichkeiten und Grenzen einer Analyse von Politikinstrumenten in der europäischen Asylforschung}

In diesem Kapitel liegt das Hauptaugenmerk darauf, sozialkonstruktivistische Ansätze der Politikinstrumentenforschung als Alternative zu funktionalistischen Ansätzen für die europäische Asylpolitikforschung vorzustellen. Dabei ist ein besonderes Interesse, die Rolle von Expertise und Expertenkollektiven in den Fokus zu nehmen. Während vorhergehende Asylforschung (vgl. Boswell 2012) sich überwiegend auf ein wissenschaftsorientiertes Expertiseverständnis konzentriert, steht demgegenüber der Vorschlag, spezialisiertes Wissen von verschiedenen Akteuren zu erfassen, welches sich auf ein bestimmtes Politikinstrument richtet. Dabei steht nicht im Vordergrund, die Konstituierung eines asylpolitischen »Feldes« zu rekonstruieren (analog zu Bigo 2014), sondern die Einflüsse von Wissen auf die Politikentwicklung anhand eines ausgewählten Politikinstrumentes der Asylpolitik erkennbar zu machen. Der Wirkungszusammenhang, der sich offenbart, zeigt, wie spezialisiertes Wissen von verschiedenen professionellen Akteuren in kondensierter Form in die Modellierung von Politikinstrumenten einfließt und dabei Repräsentationen von politischen und gesellschaftlichen Ordnungen transportiert.

Das zuvor erläuterte Beispiel EURODAC illustrierte, welche heterogenen Akteure dabei involviert waren. Experten aus der biometrischen Industrie, Innenund Sicherheitspolitik als auch Menschenrechtsgruppen und Datenschützer beteiligten sich an der Ausgestaltung und Entwicklung des Instruments. Anders als in bereits in der Asylforschung genutzten Konzepten, wie das der epistemischen Gemeinschaften, haben diese Akteure kein oder nur teilweise ein geteiltes Set an Wertvorstellungen und gemeinsamen Überzeugungen von Kausalzusammenhängen. Was sie verbindet ist, dass sie als »instrument constituencies«, als eine Art Kollektiv, ohne dabei zwingend in direkter Interaktion zu sein, durch ihre Arbeitspraktiken und ihr spezialisiertes Wissen an der Entwicklung, Verbreitung und Modifizierung eines Instrumentes beteiligt sind. Das Zusammenspiel dieser Praktiken stellt das »soziale Leben« des Instruments dar. Lascoumes und Le Galès (2007) empfehlen, die Entwicklungsverläufe von Politikinstrumenten über 
einen langen Zeitraum, die mit ihrer Entstehung einhergehenden Debatten, sowie ihre Anpassungen und Modifikationen als auch die entstehenden Kontroversen zu analysieren.

Welchen Mehrwert bringt eine sozialkonstruktivistische Perspektive auf Politikinstrumente in der europäischen Asylpolitik- und Asylverwaltungsforschung? Lascoumes und Le Galès sehen den Mehrwert der von ihnen vorgeschlagenen Analyseperspektive darin, Naturalisierungs- und Neutralisierungsprozesse von Politikinstrumenten in deren Entstehung, Einführung und Modifizierung offen zu legen. Davon versprechen sie sich, »to make visible some of the invisible - hence depoliticized - dimensions of public policies « (Lascoumes/LeGales 2007:17). Um welche Art von Naturalisierungs- und Neutralisierungsprozessen es sich dabei handelt, lässt sich anhand des vorgestellten Beispiels weiter präzisieren. EURODAC ist nach seiner Einführung nicht so grundsätzlich in Frage gestellt worden, als dass seine weitere Institutionalisierung gefährdet worden wäre. Auch wenn einzelne Mitgliedsstaaten ihre faktische Mitwirkung unterließen und es von verschiedenen Akteuren Kritik an der Ausgestaltung von EURODAC gab, entwickelte sich das Instrument mit spezifischen Eigendynamiken weiter. Die Modifizierungen und die weitere Ausgestaltung des Instruments, die eine Ausweitung der Gruppe der Datensubjekte, deren Fingerabdrücke genommen werden, sowie die Ausweitung der Zugriffsrechte auf die Datenbank beinhalteten, waren dann nicht mehr Gegenstand ausgeprägter öffentlicher politischer Kontroversen. Auch weil einzelne Kritikpunkte, wie zum Beispiel Datenschutzbedenken, berücksichtigt wurden, konnte sich das Instrument in korrigierter Form stärker manifestieren. Eine zentrale Rolle in dessen Depolitisierung spielte dabei die technische Komponente des Instrumentes. Im Fall EURODAC war das vor allem die Prägung durch die technischen Möglichkeiten der biometrischen Datenverarbeitungstechnologien. In anderen Fällen können es Kalkulationen, Berechnungen und standardisierte Prozedere sein, die als die technische Komponente des Instrumentes prägen, was durch die beteiligten Akteure als dessen technische Möglichkeiten verstanden wird. Das, was technisch machbar ist, unterstützte hier die Entwicklungsdynamik des Instrumentes. Die erheblichen Umdeutungen von den vorher eingeschriebenen politischen und gesellschaftlichen Konzeptionalisierungen blieben dabei weitestgehend implizit. Dabei wurde sowohl die schrittweise Kriminalisierung und Illegalisierung von Flüchtlingen naturalisiert, als auch die politische Dimension von identitätsbestimmenden Technologien, basierend auf Körpermerkmalen.

Ein Mehrwert dieser Perspektive lässt sich vor allem für bestimmte Instrumente in der Asylforschung annehmen. In Anlehnung an Heidbreders (2011) Unterscheidung von verwaltungsorientierten Politikinstrumenten lassen sich Ex- 
perten- und Expertisekonzepte vor allem für die nicht-hierarchischen, impliziten und indirekten Instrumente fruchtbar machen (Konformitätskontrolle und Verwaltungsanleitungen). Bei ihnen, wo nicht in erster Linie eine strikt hierarchische und sanktionierte Rechtsauslegung maßgeblich ist, kann der Blick auf Wissen die »softeren« strukturierenden Effekte offen legen. Aber auch bei den anderen Instrumenten, die hierarchisch angelegt sind, kann ein Blick auf Experten und Expertise sinnvoll sein, sofern sie eine herausragende Rolle spielen.

Begrenzungen der unterbreiteten Vorschläge liegen darin, dass die Konzepte bislang in anderen Politikbereichen Anwendung gefunden haben. Sozialkonstruktivistische Politikinstrumenteforschung wurde bislang vor allem für Beispiele aus der europäischen Umwelt-, Forschungs-, Gleichstellungs- und gemeinsamen Außen- und Sicherheitspolitik unternommen (vgl. Bruno/Jacquot/Mandin 2006; Menon/Sedelmeier 2010; Voß 2010). Das Konzept der »instrument constituencies« wurde anhand von Instrumenten in ausgewählten Politikbereichen in der Umwelt- und Sozialpolitik entwickelt und getestet. Jedoch steht der empirische Nachweis aus, für welche Politikinstrumente in der europäischen Asylpolitik und Asylverwaltung diese Konzepte besonders relevant sind. Des Weiteren sollte der jeweilige Kontext der Asylverwaltung, zum Beispiel Wissenskulturen, weiter spezifiziert werden. Gerade hinsichtlich der Verwaltungspraxis spielen Bürokratiekulturen eine wesentliche Rolle dafür, welche spezifische Bedeutung den jeweiligen Wissensformen zugeschrieben wird. Boswell hat das in ihren Fallstudien anhand der angelsächsischen und der Weberianischen Bürokratiekultur und der entsprechenden Bedeutung, die akademischer Expertise zugeschrieben wird, verdeutlicht (Boswell 2012).

Sofern dieser Beitrag sein Argument überzeugend entwickeln konnte und zur Inspiration für weitere theoretische und empirische Untersuchungen dienen kann, empfiehlt es sich, nötige konzeptionelle Modifizierungen zu erwägen und empirische Erkenntnisse über (europäische) asylpolitische Instrumente zu sammeln.

\section{Literatur}

Aas, Katja Franko (2006): ") The Body Does Not Lie $<$ Identity, Risk and Trust in Technoculture«, in: Crime, Media, Culture 2(2), S. 143-158.

Aas, Katja Franko (2011): »)Crimmigrant` Bodies and Bona Fide Travelers: Surveillance, Citizenship and Global Governance«, in: Theoretical Criminology 15(3), S. 331-346. 
Adler, Emanuel/Haas, Peter M. (1992): „Conclusion: Epistemic Communities, World Order, and the Creation of a Reflective Research Program«, in: International Organization 46(1), S. 367-390.

Amelung, Nina/Grabner, Louisa (im Erscheinen): »Making citizen panels a >universal bestseller : Transnational mobilization practices of public participation advocates«, in: Laurence Bherer/Mario Gauthier/Louis Simard (Hg.), The Professionalization of the Public Participation Field, London/New York: Routledge.

Antoniades, Andreas (2003): »Epistemic Communities, Epistemes and the Construction of (World) Politics«, in: Global Society 17(1), S. 21-38.

Aus, Jonathan P. (2006): »Eurodac: A Solution Looking for a Problem? «, in: European Integration online Papers (EIoP) 10, www.eiop.or.at/eiop/pdf/2006006.pdf

Aus, Jonathan P. (2003): »Supranational Governance in an >Area of Freedom, Security and Justices: Eurodac and the Politics of Biometric Control«, in: Sussex European Institute (SEI) Working Paper 72, www.sussex.ac.uk/ sei/documents/sei-working-paper-no-72.pdf

Béland, Daniel/Howlett, Michael (2015): »How Solutions Chase Problems: Instrument Constituencies in the Policy Process «, in: Governance (First published online), http://onlinelibrary.wiley.com/doi/10.1111/gove.12179/epdf vom 03.05.2016.

Bigo, Didier (2006): »Globalized-In-Security: The Field and the Ban-Opticon«, in: Naoiki Sakai/Jon Solomon (Hg.), Translation, Biopolitics, Colonial Difference. Hong Kong: University of Hong Kong Press, S. 109-156.

Bigo, Didier (2014): »The (in)securitization Practices of the Three Universes of EU Border Control: Military/Navy - Border Guards/Police - Database Analysts«, in: Security Dialogue 45(3), S. 209-225.

Bonelli, Laurent/ Bigo, Didier (2005): »Mapping the European Union Field of the Professionals of Security, A Methodological Note on the Problematique«, Synthesis Report of Two Seminars Organized at Science Po Paris, http://www.open.ac.uk/researchprojects/iccm/library/63.html vom 10.06.2016.

Boswell, Christina (2005) »Migration in Europe. A Paper Prepared for the Policy Analysis and Research Programme of the Global Commision on International Migration«, https:/www.iom.int/jahia/webdav/site/myjahiasite/shared/ shared/mainsite/policy_and_research/gcim/rs/RS4.pdf vom 10.06.2016.

Boswell, Christina (2012): The Political Uses of Expert Knowledge: Immigration Policy and Social Research, Cambridge: Cambridge University Press. 
Boswell, Christina/Geddes, Andrew (2011): Migration and Mobility in the European Union, Houndmills/Basingstoke/Hampshire u.a.: Palgrave Macmillan.

Bruno, Isabelle/ Jacquot, Sophie/Mandin, Lou (2006): »Europeanization through Its Instrumentation: Benchmarking, Mainstreaming and the Open Method of Co-Ordination ... Toolbox or Pandora's Box?«, in: Journal of European Public Policy 13(4), S. 519-536.

Brunsson, Nils (2002): The Organization of Hypocrisy: Talk, Decisions, and Actions in Organizations, Kopenhagen/Oslo: Abstrakt.

Bueger, Christian (2014): »From Expert Communities to Epistemic Arrangements: Situating Expertise in International Relations«, in: Maximilian Mayer/Mariana Carpes/Ruth Knoblich (Hg.), The Global Politics of Science and Technology - Vol. 1: Concepts from International Relations and Other Disciplines, Berlin/Heidelberg: Springer, S. 39-54.

Byrne, Rosemary/Shacknove, Andrew (1996): »The Safe Country Notion in European Asylum Law«, in: Harvard Human Rights Journal 9, S. 185-226.

Cross, Mai'a K. Davis (2013a): »Rethinking Epistemic Communities Twenty Years Later«, in: Review of International Studies 39(01), S. 137-160.

Cross, Mai'a K. Davis (2013b): »The Military Dimension of European Security: An Epistemic Community Approach«, in: Millennium - Journal of International Studies 42(1), S. 45-64.

Djelic, Marie-Laure/Quack, Sigrid (2010): Transnational communities: Shaping global economic governance, Cambridge: Cambridge University Press.

El-Enany, Nadine/Thielemann, Eiko R. (2011): »The impact of EU asylum policy on national asylum regimes«, in: Sarah Wolff/Flora Goudappel/Jaap W. de Zwaan (Hg.), Freedom, Security and Justice after Lisbon and Stockholm, The Hague: Asser Press, S. 97-116.

Europäische Kommission (2016): »Towards a Sustainable and Fair Common European Asylum System. Press Release (4 May 2016)«, Brüssel, http://europa. eu/rapid/press-release_IP-16-1620_en.htm

Europäisches Unterstützungsbüro für Asylfragen (2014): EASO Schulungsprogramm, Luxemburg: Amt für Veröffentlichungen der Europäischen Union.

Favell, Adrian/Guiraudon, Virginie (2011): »Sociology of the European Union: An Introduction«, in: Dies. (Hg.), Sociology of the European Union, Basingstoke: Palgrave Macmillan, S. 1-24.

Geddes, Andrew (2015): »Temporary and Circular Migration in the Construction of European Migration Governance «, in: Cambridge Review of International Affairs 28(4), S. 1-18.

Goodwin-Gill, Guy S. (1992): »Safe Country? Says Who?«, in: International Journal of Refugee Law 4(2), S. 248-250. 
Guiraudon, Virginie (2003): »The Constitution of a European Immigration Policy Domain: A Political Sociology Approach«, in: Journal of European Public Policy 10(2), S. 263-282.

Gurzu, Anca (2012): »Safe country of origin list at the EU level: The bargaining process and the implications «, in: Review of European and Russian Affairs 7(1), S. 1-15.

Haas, Peter M. (1992): »Introduction: epistemic communities and international policy coordination «, in: International organization 46(1), S. 1-35.

Hajer, Marteen A. (2006): »Doing discourse analysis: coalitions, practices, meaning«, in: Margo van den Brink/Tamara Metze (Hg.), Words matter in policy and planning: Discourse theory and method in the social sciences, Utrecht, S. 65-74.

Halpern, Charlotte (2010): »Governing Despite Its Instruments? Instrumentation in EU Environmental Policy«, in: West European Politics 33(1), S. 39-57.

Heidbreder, Eva G. (2011): »Structuring the European Administrative Space: Policy Instruments of Multi-Level Administration«, in: Journal of European Public Policy 18(5), S. 709-727.

Hood, Christopher (2007): »Intellectual Obsolescence and Intellectual Makeovers: Reflections on the Tools of Government after Two Decades«, in: Governance 20(1), S. 127-144.

Hunt, Matthew (2014): »The Safe Country of Origin Concept in European Asylum Law: Past, Present and Future«, in: International Journal of Refugee Law 26(4), S. 500-535.

Huysmans, Jef (2006): The politics of insecurity: fear, migration, and asylum in the EU, Abingdon/New York u.a.: Routledge.

Kasparek, Bernd/Tsianos, Vassilis S. (2015): »Back to the Future: Blair-Schily reloaded«, in: movements. Journal für kritische Migrations- und Grenzregimeforschung 1(1), S. 1-30.

Lascoumes, Pierre/Le Galès, Patrick (2007): »Introduction: Understanding Public Policy through Its Instruments - From the Nature of Instruments to the Sociology of Public Policy Instrumentation«, in: Governance 20(1), S. 1-21.

Linder, Stephen H./Peters, B. Guy (1990): »The design of instruments for public policy«, in: Stuart S. Nagel (Hg.), Policy Theory and Policy Evaluation: Concepts, Knowledge, Causes, and Norms, Westport, CT: Greenwood Press, S. 103-119.

Mann, Carsten/Simons Arno (2015): »Local Emergence and International Developments of Conservation Trading Systems: Innovation Dynamics and Related Problems«, in: Environmental Conservation 42(4), S. 325-334. 
Mendos Kuşkonmaz, Elif (2013): »The Eurodac Debate: Is It Blurring the Line Between Asylum and Fight Against Terrorism?«, in: Annales de la Faculté de Droit d'Istanbul 45(62), S. 79-102.

Menon, Anand/Sedelmeier, Ulrich (2010): »Instruments and Intentionality: Civilian Crisis Management and Enlargement Conditionality in EU Security Policy«, in: West European Politics 33(1), S. 75-92.

Mouzourakis, Minos (2014): ') Wrong Number?« The Use and Misuse of Asylum Data in the European Union«, in: CEPS Papers in Liberty and Security in Europe 69, http://www.ceps.eu/system/files/Use\%20and\%20Mis use \%20of\%20Asylum\%20Data.pdf vom 02.06.2015.

Pelzer, Marei (2012): »Das EuGH-Urteil vom 21.12.2011 zur >Dublin-IIVerordnung`. Keine Überstellungen bei Grundrechtsverletzungen aufgrund systematischer Mängel«, in: Asylmagazin 4, S. 102-108.

Ploeg, Irma van der (1999): »The illegal body: ১Eurodac and the politics of biometric identification«, in: Ethics and Information Technology 1(4), S. 295-302.

Sabatier, Paul A./Jenkins-Smith, Hank C. (1993): Policy change and learning: An advocacy coalition approach, Boulder, CO: Westview Press.

Schuster, Liza (2011): »Dublin II and Eurodac: Examining the (un)intended(?) Consequences«, in: Gender, Place \& Culture 18(3), S. 401-416.

Tholen, Berry (2010): »The Changing Border: Developments and Risks in Border Control Management of Western Countries «, in: International Review of Administrative Sciences 76(2), S. 259-278.

Thym, Daniel (2013): »Zulässigkeit von Dublin-Überstellungen nach Italien«, in: Zeitschrift für Ausländerrecht und Ausländerpolitik 33, S. 331-334.

Tömmel, Ingeborg (2014): Das Politische System der EU, München: Oldenbourg Wissenschaftsverlag.

Töpfer, Eric (2015): »Ein Ding, sie zu finden... Eurodac und die biometrische Erfassung asylsuchender und irregulärer Migranten«, in: Datenschutz Nachrichten 2, S. 64-68.

Trauner, Florian/Wolff, Sarah (2014): »The Negotiation and Contestation of EU Migration Policy Instruments: A Research Framework«, in: European Journal of Migration and Law 16(1), S. 1-18.

Tsianos, Vassilis S. (2015): »Die (Un-)Durchlässigkeit der europäischen Außengrenzen für Geflüchtete. Der Fall Eurodac«, in: Soziale Probleme 26(2), S. 189-204.

Velutti, Samantha (2014): Reforming the Common European Asylum System Legislative Developments and Judicial Activism of the European Courts, New York: Springer. 
Voß, Jan-Peter (2007a): Designs on Governance. Development of Policy Instruments and Dynamics in Governance, Enschede: University of Twente, http://doc.utwente.nl/58085/1/thesis_Voss.pdf vom 04.03.2010.

Voß, Jan-Peter (2007b): »Innovation processes in governance: the development of >emissions trading« as a new policy instrument «, in: Science and Public Policy 34(5), S. 329-343.

Voß, Jan-Peter (2010): »Innovation of Governance: the Case of Emissions Trading «, in: Maarten J. Arentsen/Wouter van Rossum/Albert E. Steenge (Hg.) Governance of Innovation. Firms, Clusters and Institutions in a Changing Setting, Edward Elgar, S. 125-148.

Voß, Jan-Peter/Simons, Arno (2014): »Instrument Constituencies and the Supply Side of Policy Innovation: The Social Life of Emissions Trading «, in: Environmental Politics 23(5), S. 735-754.

Woodward, Susan L. (2004): »Enhancing Cooperation against Transborder Crime in Southeast Europe: Is There an Emerging Epistemic Community? «, in: Southeast European and Black Sea Studies 4(2), S. 223-240. 


\section{«Asyl fängt ja erst an, wenn er [der Flüchtling] wirklich hier ist "}

Der Wandel europäischer Visapolitik und seine Implikationen für den Zugang zu Asylverfahren in der EU

LENA LAUBE

\section{Einleitung}

Das Jahr 2015 hat Europa einen sprunghaften Anstieg der Flüchtlingsmigration gebracht. Rund eine Million MigrantInnen sind allein in Deutschland angekommen. ${ }^{1}$ Zum allergrößten Teil werden sie um Asyl ersuchen, da sie nur so eine Chance auf einen legalen Aufenthalt oder zumindest eine Aufenthaltsgenehmigung während dieses Verfahrens haben. Das Bundesamt für Migration und Flüchtlinge (BAMF) war plötzlich in aller Munde, die Dauer der Asylverfahren wurde von Politik und Medien als zu lang kritisiert. ${ }^{2}$ Durch die Einstellung von mehr Personal und die Verkürzung von Antragsbearbeitungen, z.B. durch die Festlegung weiterer sicherer Herkunftsländer ${ }^{3}$, wird nun versucht, dieser Kritik zu begegnen und die Bearbeitungszeiten zu beschleunigen. Ein weiterer Fokus der aktuellen Debatte liegt auf den EU-Außengrenzen, vor allem am Mittelmeer. Dort kamen in den vergangenen Jahren Hunderttausende Flüchtlinge und Mig-

$1 \mathrm{http} / / /$ www.sueddeutsche.de/politik/deutschland-mehr-als-eine-million-fluechtlinge1.2755884 vom 1.12.2015.

2 http://www.zeit.de/politik/deutschland/2015-12/asyl-bamf-verwaltung-ueberforderung -asylantraege vom 10.12.2015.

3 Siehe unter anderem https://www.proasyl.de/wp-content/uploads/2016/02/160129_ Referentenentwurf_Maghrebstaaten_sichere_HKS.pdf vom 17.6.2016. 
rantInnen mit kaum seetüchtigen Booten an, den Strapazen und Gefahren der Überfahrt nur knapp entgangen. Sehr viele starben auch bei der Überfahrt. Der traurige Höhepunkt dieser Situation wurde nach Angaben des UNHCR im April 2015 erreicht, als allein in diesem einen Monat über 1.300 Menschen ihr Leben bei der Überfahrt nach Europa verloren. ${ }^{4}$ Dieser humanitären Tragödie versuchen die europäischen Staaten inzwischen mit Seenotrettungen und weiteren Kontrollen auf hoher See zu begegnen. Auch sollen Kampagnen in den Herkunftsländern klarer machen, wie groß die Gefahren sind und wie klein dagegen die Chance, einen gesicherten Aufenthalt in Deutschland und Europa zu erhalten. ${ }^{5}$ So wird etwa unter dem »\#rumorsaboutgermany « in Kabul auf Plakaten darauf hingewiesen, dass die Aufnahmepolitik Deutschlands gegenüber Afghanen bei weitem nicht so generös ist, wie sich dies zeitweilig als Gerücht in den sozialen Medien des Landes verbreitet hatte. ${ }^{6}$

Lange im Schatten dieser Medienberichte trat 2016 eine der wohl wichtigsten Fragen der derzeitigen europäischen Grenzpolitik zu Tage: Mit welchem Entgegenkommen kann die Türkei in der eben geschilderten Lage dazu gebracht werden, sich als Gatekeeper für die Europäische Union anzudienen? In den dazugehörenden Verhandlungen kann die EU kurzfristig tätig werden, während es doch weitgehend außerhalb ihrer Macht steht, die Fluchtursachen in den kriegsund krisengeschüttelten Ländern des Nahen und Mittleren Osten zu beheben. Entsprechend einem Abkommen zwischen der EU und der Türkei sollen diejenigen Personen in Flüchtlingslagern in der Türkei verbleiben, die z.B. aus dem Irak, Iran und Afghanistan geflohen sind. Wenn dieser Personenkreis versucht, über die Ägäis nach Griechenland zu fliehen, verpflichtet sich die Türkei zur Rücknahme (seit März 2016 umgesetzt). Doch die Türkei stellte hohe Forderungen im Gegenzug zu einer Kontrolle der Migrationsbewegungen gen Westeuropa und für die Aufnahme so vieler MigrantInnen. Das Abkommen sieht daher finanzielle Hilfen in Milliardenhöhe sowie die Aufnahme von syrischen Flüchtlingen aus der Türkei in die EU vor. Kommt es zur vollständigen Ratifizierung des

4 http://www.unhcr.de/home/artikel/435da63ac57f3eaee63d95d2e4f7eb9b/mittelmeerrekordzahl-von-fluechtlingen-und-migranten.html vom 1.12.2015.

5 Solche Kampagnen werden u.a. in Afghanistan initiiert. Doch auch in Albanien und Serbien gibt es ähnliche Informationskampagnen, um die Nachricht zu verbreiten, wie klein die Anerkennungsquote bei Asylanträgen in Deutschland ist, http://www.zeit.de/ politik/deutschland/2015-08/fluechtlinge-asyl-albanien-serbien-bundesamt vom 1.12. 2015.

6 http://www.reuters.com/article/us-europe-migrants-germany-embassies-idUSKCN 0RE1 JM20150914 vom 1.12.2015. 
Abkommens, steht auch eine erleichterte Einreise für Türken in die EU in Aussicht, unter Umständen sogar die sofortige Aufhebung der Visumspflicht für den gesamten Schengenraum. Doch die Einführung der Visumsfreiheit ist an 72 formulierte Bedingungen seitens der EU geknüpft worden und eine erste Frist (1.6.2016) für deren Erfüllung bereits verstrichen. ${ }^{7}$

Die Visumsfreiheit ist das Pfand, das die EU (noch) in der Hand hält und welches sie nicht allzu schnell bereit ist aus der Hand zu geben. Sie kann den BürgerInnen eines Transitlandes das visafreie Reisen ermöglichen, um eben dieses Land dazu zu bewegen, MigrantInnen und Flüchtlingen aus anderen Drittstaaten, die der Visumspflicht unterliegen, die Durchreise zu verwehren. Andernfalls würden diese in die europäischen Asylsysteme einwandern. An dieser Stelle sind Asyl- und Visapolitik in den aktuellen politischen Verhandlungen aufs engste verwoben.

Doch nicht nur in solchen Verhandlungen sind diese beiden Politikfelder eng verzahnt. Auch für jeden einzelnen Asylsuchenden besteht dieser Zusammenhang. Denn die europäischen Asylgesetze sehen vor, dass um Asyl erst an der Grenze oder auf dem Territorium des schutzgebenden Landes ersucht werden kann. Dies bedeutet, dass die An- bzw. Einreise in das betreffende Land der EU möglich sein muss, um überhaupt einen Asylantrag stellen zu können. Besteht Visumsfreiheit bzw. Reisefreiheit für die BürgerInnen aus einem Verfolgerstaat in das Land, in dem Schutz gesucht werden soll, so kann ein Flüchtling legal einreisen und einen Asylantrag stellen. Besteht eine Visumspflicht, muss er oder sie mit der Ablehnung schon des Visumsantrages rechnen und sich ggf. illegal auf den Weg machen, bevor sich dann vor Ort klären kann, ob das Zielland seine Verfolgungsgründe anerkennt und Schutz gewährt. Dass die Asylverfahren erst

7 http://www.zeit.de/politik/ausland/2016-05/eu-tuerkei-abkommen-fluechtlinge-receptayyip-erdogan-visafreiheit vom 17.6.2016. Das als »Deal« oder »Flüchtlingspakt« bezeichnete EU-Türkei-Abkommen steht in Europa in der öffentlichen Kritik, weil eine Abhängigkeit von der Türkei in migrationspolitischer Hinsicht zementiert würde. Die Delegation von Migrationskontrolle muss teuer erkauft werden und bei einem Partner wie der Türkei zeigte sich schnell, dass eine solche Kooperation auf wackligen Beinen stehen kann (vgl. Laube/Müller 2015): Der Ministerpräsident, der zu Beginn die Verhandlungen führte (Davutoğlu), gab sein Amt im Mai 2016 auf und Präsident Erdoğan versteigt sich zu immer neuen Drohungen und Anschuldigen gegenüber der EU. 
im Aufnahmeland stattfinden ${ }^{8}$, führt bei politischen AkteurInnen zu der verbreiteten Sichtweise, dass zuvor keine Zuständigkeit des Ziellandes besteht:

»Asyl fängt ja erst an, wenn er [der Flüchtling, Anmerkung L.L.] wirklich hier ist. Das was vorher passiert, hat ja vor allem mit der klassischen Genfer Konvention nichts zu tun.«(Interview Österreich 2009, BMEIA, \#11)

Eine restriktive Visapolitik kann somit den Zugang zu einem Asylverfahren erschweren oder gar verhindern. Umgekehrt ist eine liberale Visapolitik, also die Gewährung des visafreien Reisens, der sicherste Weg, den Zugang für politisch Verfolgte offenzuhalten. Doch oft sind gerade diejenigen Länder von der Visumspflicht befreit, aus denen keine oder kaum Asylbewerber kommen und wiederum gerade diejenigen Länder von der Visumspflicht betroffen, aus denen Personen kommen, die Schutz benötigen und in Europa suchen. Visapolitik entfaltet dabei ihre Kontrollwirkung exterritorial, denn sie erschwert Mobilität oder immobilisiert Asylsuchende bereits in ihrer Herkunftsregion.

Wandelt sich nun die Visapolitik der aufnehmenden Staaten, hat dies direkte Auswirkungen auf die Möglichkeiten, um Asyl zu ersuchen, entweder für die BürgerInnen des betreffenden Landes oder aber für Drittstaatler, die durch dieses Land reisen müssen. Dies betrifft vor allem den territorialen Zugang zum Asylverfahren, aber auch die Chancen, im Verfahren anerkannt zu werden. Wird ein Herkunftsstaat in den Stand eines Landes gehoben, das keiner Visumspflicht (mehr) unterliegt, beruht dies auf der allgemeinen politischen Einschätzung, dass dieses Herkunftsland vergleichsweise sicher ist und die bilateralen Beziehungen stabil sind. Etabliert sich eine solche Sichtweise, kann dies Auswirkungen auf die Chancen einer politisch verfolgten Person aus diesem Land im Asylverfahren haben.

8 Eine Ausnahme zu dieser Regel stellen Resettlement-Programme dar. In diesen werden in den Erstaufnahmeländern Flüchtlinge ausgesucht, denen ein Aufenthaltsrecht im Zielland direkt zugesagt wird. Deutschland hat seit 2012 ein solches Programm, über das zunächst jährlich 300 Flüchtlinge, seit 2015500 Personen, die zu diesem Zeitpunkt ersten Schutz in der Türkei oder Tunesien gefunden hatten, aufgenommen werden. (Siehe http://www.bmi.bund.de/DE/Themen/Migration-Integration/AsylFluechtlingsschutz/Humanitaere-aufnahmeprogramme/humanitaere-aufnahmepro gramme_node.html vom 9.12.2015). Auch das Nachholen von Familienangehörigen durch Asylberechtigte entspricht nicht der oben genannten Regel. Österreich etwa bietet seit 1997 ein solches »Familienverfahren« an (Interview Österreich, 2009, BMI, $\# 13)$. 
Dieser Beitrag untersucht, welchen Wandlungen die europäische Visapolitik in den vergangenen Dekaden unterlag und welche Auswirkungen dieser Wandel auf den Zugang zu den Asylverfahren in der EU hatte. Verändern sich dabei auch die Chancen auf Anerkennung? Als Beispiele für die aufnehmenden Länder werden für diesen Beitrag im besonderen Deutschland und Österreich herangezogen. Da seit 2001 die Visapolitik der Europäischen Union harmonisiert ist, betrifft die Analyse ab diesem Zeitpunkt auch die EU als Ganze. Aus Österreich stammt ein Großteil der Experteninterviews, die helfen werden, den Zusammenhang zwischen Visa- und Asylpolitik in diesen Beitrag zu veranschaulichen. ${ }^{9}$

Um die Implikationen des Politikwandels im Bereich Visa für die Herkunftsländer zu verdeutlichen, werden auch dafür konkrete Länderbeispiele herangezogen. Ausgewählt wurden dafür die Türkei sowie die Nachfolgestaaten des ehemaligen Jugoslawien. Diese liegen alle in der europäischen Peripherie und aus ihnen stammt ein Großteil der MigrantInnen in Österreich und Deutschland. Die meisten Westbalkanstaaten unterliegen bereits keiner Visumspflicht mehr in der EU, nicht so die Türkei. Aus diesem Land stammten 2012 vier Prozent aller Anträge für ein Schengenvisum. ${ }^{10}$ Wie das Eingangsbeispiel gezeigt hat, spielen

9 Die Experteninterviews wurden für ein Forschungsprojekt des Sonderforschungsbereichs 597 »Staatlichkeit im Wandel« der Universität Bremen erhoben (Leitung Prof. Steffen Mau). In diesem Projekt wurde zwischen 2007 und 2014 die Grenzpolitik in drei OECD-Staaten untersucht (Österreich, Finnland und die USA). Die Analysen des österreichischen Falls zeigen ein Land, das sich zwischen 1970 und heute zu einem Einwanderungsland gewandelt hat und in seiner Grenzpolitik Teil des Schengener Abkommens wurde. Die für diesen Beitrag verwendeten leitfadengestützten Interviews (vgl. Bogner/Littig/Menz 2005; Witzel/Reiter 2012) wurden 2008 und 2009 von der Autorin in Wien geführt. Interviewpartner waren Ministerialbeamte, die in den zuständigen Ministerien in leitender Funktion mit Zuwanderungs- und Asylfragen betraut waren. Die Interviews dienen hier zur Veranschaulichung des Zusammenhangs von Asyl- und Visapolitik. Eine ausführliche qualitative Analyse des gesamten Interviewmaterials findet sich in der Studie »Grenzkontrollen jenseits nationaler Territorien « (Laube 2013). Weitere Ergebnisse der drei Länderstudien wurden unter anderem in dem Band »Liberal States and the Freedom of Movement. Selective Borders, Unequal Mobility « (Mau et al. 2012) veröffentlicht.

10 Damit nimmt die Türkei Platz 5 der Herkunftsländer ein, vor ihr nur Russland, die Ukraine, China und Weißrussland. Siehe http://ec.europa.eu/dgs/home-affairs/whatwe-do/policies/borders-and-visas/visa-policy/docs/overview_of_schengen_visa_ statistics_en.pdf vom 28.01.2016. 
diese Staaten zudem gegenwärtig eine besondere Rolle in der Migrationskontrollstrategie der EU.

Der Beitrag verfolgt die These, dass die Visapolitik der europäischen Staaten untereinander bzw. gegenüber Staaten außerhalb der europäischen Gemeinschaft seit dem Ende des Zweiten Weltkrieges zwei Phasen durchlaufen hat und nun im Begriff ist, in eine dritte Phase einzutreten. Diese drei Phasen werden im Folgenden umrissen und ihre Implikationen für den Zugang zu Asylverfahren in der EU reflektiert. Für die Analyse dieser Großtrends der internationalen Visapolitik wird ein selbst erstellter Datensatz verwendet (Visa Network Data 1969/2010), der für zwei Zeitpunkte sämtliche Informationen darüber bereithält, zwischen welchen zwei Ländern weltweit visafreies Reisen bestand und zwischen welchen Staaten das Reisen nur nach vorheriger Visumsbeantragung erlaubt war. ${ }^{11}$

\section{Drei Phasen der Visapolitik in Europa}

Die Visapolitik ist Teil der europäischen Grenzregime, da mit ihrer Hilfe über den Zugang von anderen Staatsbürgern auf das Territorium der europäischen Nationalstaaten sowie dem Schengenraum als Ganzem entschieden wird (vgl. Guild 2003; Bigo/Guild 2005; Luedtke/Byrd/Alexander 2010; Mau/Brabandt 2011). Obwohl Gesetze zur Sichtvermerksfreiheit oder -pflicht bereits länger existieren als die Asyl- oder Zuwanderungsgesetzgebungen, wird ihnen von der Grenzregimeforschung bisher sehr wenig Beachtung geschenkt (vgl. Eigmüller/Vobruba 2006; Hess/Kasparek 2010; Geiger/Pécoud 2013). Der Beitrag möchte dies ändern und versucht diese Lücke in der Grenzregimeforschung zu schließen. Ziel ist es dabei, den politischen Umgang in Europa mit Drittstaatlern, ob MigrantInnen, TouristInnen oder Flüchtlingen, besser zu verstehen.

Dabei stellt die Visapolitik ein sehr vielversprechendes sozialwissenschaftliches Forschungsfeld dar, sowohl für die Grenzregimeforschung und die Migrationsstudien, gerade auch mit Blick auf Asylsuchende, als auch für die Internationalen Beziehungen (IB). Denn die Visapolitik berührt immer zugleich die Beziehung zwischen zwei Staaten (Verbündete, Konkurrenten, Gegner?) und regelt die Mobilität von Individuen (erwünscht/unerwünscht?; viele/wenige?). Auf dieser zweiten Ebene, auf der es um Fragen der Inklusion geht, stehen sich also ein

11 Der Datensatz sowie ausführliche Angaben zu Erhebung lassen sich auf folgender Website einsehen: www.fiw.uni-bonn.de/demokratieforschung/personen/laube/visanetworkdata. 
Staat als Zielland und ein Individuum, und zwar als Staatsbürger eines anderen Landes, gegenüber.

Die drei im Folgenden skizzierten Phasen des Wandels der europäischen Visapolitik verbinden sich mit je einer These:

- These $1 » V i s a f r e i h e i t$ als Geschenk«: Die Visapolitik europäischer Länder war nach dem zweiten Weltkrieg auf Freiheit ausgerichtet. Mit der Aufhebung der sonst geltenden Visumspflicht wurde Partnern und Verbündeten ein positives Signal gesendet und der Austausch erleichtert.

- These $2 » V i s a p f l i c h t$ als Hürde zum Asyl«: Die europäische Visapolitik wurde seit den 1990er Jahren immer stärker zu einem Migrationskontrollinstrument ausgebaut, das auch den Zugang zu Asylverfahren in den EUMitgliedstaaten blockierte.

- These $3 » V i s a f r e i h e i t$ als Tausch«: Seit einigen Jahren wird die Befreiung von der Visumspflicht als gewichtiges Pfand in Verhandlungen der EU mit Nachbarländern genutzt: Nur unter bestimmten Bedingungen kann Reisefreiheit erreicht werden und diese Länder gelten dann als sicher.

Auf der Beziehungsebene besinnt sich die europäische Visapolitik gegenwärtig wieder stärker darauf, ein außenpolitisches Instrument in der Kooperation mit anderen Staaten zu sein, statt sich - wie in Phase zwei - zuallererst als Kontrollinstrument gegen unerwünschte grenzüberschreitende Mobilität von Individuen zu verstehen.

\subsection{Visafreiheit als Geschenk (1950 bis 1990)}

In der Visapolitik besteht der Normalfall darin, dass BürgerInnen zwischen zwei Ländern nur nach vorheriger Visabeantragung und -bewilligung durch das Zielland reisen dürfen. ${ }^{12}$ Eine Befreiung von dieser Visapflicht müssen Staaten untereinander regeln. Dies erfolgt zumeist in einem bilateralen Vertrag, kann aber auch einseitig erfolgen, sodass die BürgerInnen eines anderen Landes ohne Beschränkungen einreisen dürfen, dieses Privileg aber den eigenen BürgerInnen umgekehrt nicht zugestanden wird. Ein Bespiel hierfür ist das visabefreite Reisen von Deutschen nach Albanien, während AlbanerInnen vor der Einreise nach

12 Betrachtet werden in diesem Beitrag Visa, die zu kürzeren Aufenthalten (als internationaler Standard gilt hier drei Monate) berechtigen und zum Zweck von Tourismus, Geschäftsreisen, Forschungsaufenthalten, Familienbesuchen u.ä. vergeben werden. Gemeint sind damit keine längerfristigen Aufenthaltstitel. 
Deutschland und in den Schengenraum ein Visum für einen Aufenthalt von drei Monaten beantragen müssen. ${ }^{13}$

In der ersten Phase der Visapolitik nach dem Zweiten Weltkrieg gestalteten die europäischen Staaten, wie auch alle anderen Staaten der Erde, ihre Visabeziehungen zu anderen Ländern einzelstaatlich. Der Korpus der Visabestimmungen eines Landes setzt sich zusammen aus entsprechend vielen Übereinkommen mit anderen Staaten (für das Beispiel Österreich siehe Laube 2013). In den 1950er Jahren begannen die westeuropäischen Länder zunächst damit, Abkommen mit ihren direkten Nachbarländern zu unterzeichnen, die beinhalteten, dass die Bürger beider Länder ohne ein Visum und mehrfach im Jahr ein- und ausreisen durften. So gehörten die »Abkommen über die Aufhebung der Visumspflicht« zwischen Deutschland und der Schweiz sowie Österreich zu den ersten Visabefreiungen aus deutscher Sicht. Dieses Vorgehen weiteten Deutschland und auch Österreich im Laufe dieser Phase auf weitere westeuropäische Länder aus, die Staaten des Ostblocks wurden jedoch nicht einbezogen. Während des kalten Krieges determinierte die politische Blockzugehörigkeit eindeutig die Visapolitik (vgl. Laube/Heidler 2016).

Im Jahr 1969 durften die BürgerInnen aus insgesamt 92 Ländern visafrei in die Bundesrepublik einreisen. Österreich hatte 50 andere Staaten von der Visapflicht befreit. Für die direkten europäischen Nachbarn bildeten diese Befreiungen den ersten Schritt hin zu einem gemeinsamen Wirtschaftsraum. Arbeitnehmer, Geschäftsleute und Touristen konnten seither frei reisen, auch wenn noch kein Recht auf Niederlassung zum Zweck der Arbeitssuche bestand.

Auch die Türkei (als bereits damaliges Nato-Mitglied) und das ehemalige Jugoslawien (als Teil der Blockfreien Staaten) genossen Ende der 1960er Jahre visabefreites Reisen nach Deutschland und Österreich (Visa Network Data 1969). Sie gehörten damit zu den Ländern, die von einer Visapolitik, die in Westeuropa als Geschenk verstanden wurde, profitierten. So konnte die damalige Gastarbeitermigration zunächst ohne weitere Beschränkungen zur Einreise erfolgen. Erst nach der Ankunft wurde über einen längerfristigen Status entschieden.

Doch wie erklärt es sich, dass Deutschland (wie auch andere europäische Staaten) zu dieser Zeit ebenfalls bilaterale Abkommen über die Visabefreiung mit Ländern wie Angola, Kamerun, Kolumbien, Libyen, Mozambik und Venezuela hatte? Der Grund sind Auslandsbesuche deutscher Politiker oder Diplomaten, die diese Abkommen als Zeichen guten Willens in den zwischenstaatlichen

13 Siehe Travel Information Manual von der International Air Transport Association (IATA) (März 2014). 
Beziehungen mit im Gepäck hatten (vgl. Laube 2013). Ein Ministerialbeamter des österreichischen Außenministeriums beschrieb in einem Experteninterview die Politik der Visabefreiungen in den 1970er und 1980er Jahren als relativ zufälligen Prozess, der den außenpolitischen Beziehungen diente:

»Da wurde bei irgendeinem politischen Treffen oder Staatsbesuchen vereinbart, dass das gemacht wird. Damals war Visumspolitik eher der Anhang zur allgemeinen Politik und wenn man sonst nichts zu unterschreiben hatte, dann gab es eine Sichtvermerksfreiheit.« (Interview Österreich 2009, BMEIA, \#11)

Diese Praxis der Visabefreiung (Sichtvermerksfreiheit) als rein symbolischer Akt verweist darauf, dass zu dieser Zeit nicht die Annahme bestand, dass das visafreie Reisen von Angolanern oder Kamerunern sich merklich auf die Einwanderung in Länder wie Deutschland oder Österreich auswirken würde. »Wenn man sonst nichts zu unterschreiben hatte « war auch die Befreiung von der Visumspflicht keine große Sache. Die Asylantragszahlen waren zu dieser Zeit ohnehin niedrig. Zudem war die Asylgewährung ein politisches Instrument, um sich gegen die Politik anderer Länder zu richten. So nahm die BRD gern politische Flüchtlinge aus den damaligen Ostblockstaaten auf, als außenpolitisches Signal sowie nach innen als Zeichen der Abgrenzung gegenüber einem politischen System, das nicht den eigenen Werten entsprach. Zwischen 1953 und 1973 etwa lagen die Antragszahlen pro Jahr unter 6.000, einzige Ausnahme bildeten die Jahren 1955/56 (mehr als 16.000) und 1968/1969 (mehr als 11.000), in denen nach Aufständen in Polen und Ungarn sowie dem »Prager Frühling« eine größere Zahl politischer Flüchtlinge in die Bundesrepublik gekommen war.

Die Verbindung zwischen Visapolitik und Asylpolitik war in dieser Phase eine nur sehr lose. Mit den Staaten, aus denen die meisten Asylanträge stammten, hatten die BRD oder Österreich zu dieser Zeit keine Befreiungen von der Visumspflicht vereinbart. Gelang einer Person, z.B. aus dem Ostblock, die Einreise und wurde ein Asylantrag gestellt, so hatte diese gute Chancen auf Anerkennung. Man kann in dieser Hinsicht also auch von einem negativen Zusammenhang sprechen: Länder, aus denen politisch Verfolgte kamen, kamen für ein Abkommen über die Aufhebung der Visumspflicht nicht in Frage. Wie sich im Weiteren zeigen wird, entfaltet bis heute der Umkehrschluss (aus visumsbefreiten Staaten stammen in der Regel keine Asylberechtigten) seine Wirkmächtigkeit in der Wahrnehmung von Flüchtlingen. 


\subsection{Visapflicht als Hürde zum Asyl (1990 bis 2000er)}

Die Handhabung der Visapolitik veränderte sich in Europa seit den 1990er Jahren beständig in Richtung einer immer restriktiveren Zulassungspolitik (vgl. Mau et al. 2012; Laube 2013; Mau et al. 2015). Einen wichtigen Aspekt dieses Wandels stellte die Vergemeinschaftung der Visumspolitik in der Europäischen Union dar, welche auf die Abschottung vor »unerwünschten« Migrationsbewegungen ausgerichtet war. Nach jahrelangen Verhandlungen einigten sich die EUStaaten in der Verordnung (EG) 539/2001 auf eine Liste von Staaten, deren BürgerInnen bei der Einreise in alle Mitgliedsländer der EU im Besitz eines gültigen Visums sein müssen. Alle übrigen Länder sollten für die gesamte EU von der Visumspflicht befreit sein. Diese gemeinsame, außenpolitische Linie war die Basis für die Einführung eines einheitlichen Schengenvisums und standardisierter Verfahren zu seiner Vergabe (vgl. Interview Österreich 2009, \#12 und \#13). Durch diese gemeinsame Liste waren fast alle EU-Staaten dazu verpflichtet, einigen Drittstaaten die Befreiung von der Visumspflicht wieder zu entziehen. Dazu gehörten, soweit die Abkommen noch Bestand hatten, die oben genannten Staaten wie Angola oder Kamerun, aber auch Länder der EU-Peripherie wie die Türkei oder zunächst Bosnien, Serbien und Mazedonien. Seither müssen unter anderen türkische StaatsbürgerInnen für die Einreise in alle Länder der EU ein Schengen- oder nationales Visum beantragen.

Generell lag der Schwerpunkt der europäischen und damit zugleich der deutschen bzw. österreichischen Visapolitik in dieser Phase auf der Visumspflicht. Hintergrund dafür ist die Annahme der politischen Akteure, dass eine Aufhebung der Visumspflicht für einen Drittstaat nicht nur - wie beabsichtigt - die kurzfristige grenzüberschreitende Mobilität (Tourismus, Familienbesuche, Grenzpendler) verändert, sondern auch das allgemeine Zuwanderungsgeschehen (vgl. Parusel/Schneider 2012). Die Möglichkeit, als Tourist einzureisen und dann vor Ort einen Asylantrag zu stellen oder anderweitig dauerhaft zu bleiben, sollte erschwert werden. Es sollten nur solche Länder von einer Visabefreiung profitieren, aus denen die Zielländer keine oder nur selten Asylanträge erwarteten. Die Visapolitik wurde daher als Instrument gegen mögliche Migrationsbewegungen in Stellung gebracht. Vor allem sollte der Zuzug von Asylsuchenden erschwert werden.

Ob eine restriktivere Visapolitik jedoch den gewünschten Effekt hat und dazu beiträgt, die Asylantragszahlen zu reduzieren, ist bisher in der Literatur umstritten. Während einige empirische Studien zeigen, dass ein Wandel in den Visa- und Asylpolitiken zu einer Verringerung neuer Asylanträge führen kann (vgl. Neumayer 2004; Thielemann 2006), weisen andere Autoren darauf hin, dass es 
neben diesem »Abschreckungseffekt« auch einen »deflection effect« (Umlenkungseffekt) geben kann (vgl. Czaika/Hobolth 2014). Dies bedeutet, dass Schutzsuchende, die kein Visum erhalten, sich stattdessen auf illegale Wege begeben. Unter Umständen meiden sie in der Folge im Zielland auch das Asylverfahren und nehmen direkt ein Leben in der Illegalität in Kauf. Czaika und Hobolth (2014) kommen am Beispiel von 29 europäischen Ländern zu dem interessanten Ergebnis, dass die Verschärfung von Asylgesetzen zwar die Antragstellungen zahlenmäßig reduzieren kann. Doch dieser gewünschte Effekt wird ausgeglichen bzw. zunichte gemacht, da es einen gleichzeitigen Anstieg der Zahl irregulärer Einreisen und Aufenthalte gibt. Einen deutlicheren reduzierenden Effekt auf die Anzahl von Asylanträgen finden sie in ihrer Studie hingegen, sofern ein Herkunftsland der Visapflicht unterliegt (Czaika/Hobolth 2014: 19).

Asylsuchende sind in besonderer Weise von der Visumspflicht ihrer Herkunftsländer betroffen. Da diese Politik sich immer systematisch an alle BürgerInnen eines Landes richtet, wird keine Rücksicht auf besondere persönliche oder politische Umstände genommen. Zwar ist die Exklusion politischer Flüchtlinge nicht offizielles Ziel von Visapolitik, dies kann aber leicht die Folge sein (Noll 2000: 161).

Eine Visumspflicht erschwert den Zugang zu einer Asylantragstellung in vielerlei Hinsicht (vgl. Guiraudon 2003; Lax 2008; Laube 2013). So entstehen etwa finanzielle Kosten und zeitlicher Aufwand bei der Antragstellung. Nicht selten liegt die Botschaft oder das nächste Konsulat eines Ziellandes weit entfernt, z.B. in der Hauptstadt oder sogar in einem Nachbarland (für den Fall europäischer Botschaften in Afrika, siehe Laube 2013, S. 159-160). Dorthin müssen AntragstellerInnen reisen, da man für den Erwerb z.B. eines Schengenvisums nicht nur Dokumente einreichen, sondern auch persönlich in der konsularischen Vertretung erscheinen muss (Verordnung (EG) 810/2009, Visakodex, Absatz 9). Dieser Aufwand kann für politisch Verfolgte bereits zu groß, zu gefährlich oder zu langwierig sein. Wird dennoch ein Visaantrag gestellt, heißt dies noch nicht, dass er auch bewilligt wird und die legale Einreise in einen schutzgebenden Staat erfolgen kann. Beispielhaft konnte eine Studie unter iranischen Asylbewerbern in den Niederlanden (vgl. Koser 2000) aufzeigen, dass viele zunächst versucht hatten, ein Visum zu beantragen und legal einzureisen. Im Falle der Ablehnung waren sie dann aber auf illegalem Weg, zumeist unter Zuhilfenahme von Schleppern, in die Niederlande gelangt und hatten dann dort einen Asylantrag gestellt. ${ }^{14}$

14 Darüber, ob auch viele politisch Verfolgte nach einem Visaablehnungsbescheid im Land verbleiben oder es mit einem anderen Zielland versuchen, kann die Studie von 
Dieses Abdrängen Vieler auf illegale Arten der Einreise kann selten beachtete, aber für die Betroffenen schwerwiegende Folgen haben. So kann bei politischen AkteurInnen wie Politikern, Ministerialbeamten oder auch Asylentscheidern die Annahme entstehen, dass derjenige, der auf legalem Weg einreist, kein echter politischer Flüchtling sein kann. In diesem Sinne führte ein Beamter des österreichischen Außenministeriums aus:

»Zum einen hat der [Asylsuchende, L.L.] in der Regel ja auch andere Fluchtmöglichkeiten. Wenn jemand wirklich von seinem Heimatstaat verfolgt wird, dann wird ihm das fast unmöglich, ins Flugzeug zu kommen. Der passiert einen Polizisten bei der Ausweiskontrolle und ich glaube kaum, dass ein unerwünschter Regimekritiker es überhaupt schaffen würde, diesen [Polizisten, L.L.] zu passieren. Ich glaube nicht, dass man hier etwas einschränken muss, denn wenn jemand es schafft, ein Flugzeug zu besteigen, kann der Verfolgungsgrad nicht so hoch sein. Abgesehen davon setzt das klassische Asylrecht wirklich erst mit dem Eintreffen ein.«(Interview Österreich 2009, BMEIA, \#11)

Die Argumentation in dieser Interviewpassage zeigt auf, dass sich die Sichtweise der beteiligten AkteurInnen auf Asylsuchende entsprechend der Zulassungspolitiken der Aufnahmeländer, aber auch entsprechend der Ausreisepolitik der Herkunftsländer verändern kann. Der Experte im Außenministerium legt nahe, sich bei der Beurteilung von Einzelfällen auch an den Ausreisekontrollen des Heimatlandes zu orientieren: Wer ohne Schwierigkeiten per Direktflug in Österreich ankommt, steht zumindest unter dem Verdacht, keinen ausreichenden Grund und Anspruch auf Asyl wegen politischer Verfolgung zu haben. Hier zeigt sich ein bedeutsamer Zusammenhang zwischen den individuellen Bedingungen einer Einreise und den zugestandenen Chancen auf Anerkennung als Schutzsuchendem.

Wie wir sehen, ergibt sich in dieser Phase, in der die Visapolitik als Hürde dient, eine deutlich engere Verknüpfung zwischen Asyl- und Visapolitik. Nachdem die Einwanderung nach Westeuropa seit den 1980er Jahren deutlich angestiegen war und sich immer mehr diversifizierte, wurde auch die Sorge laut, der Zugang zu einem Asylantrag könne von Einwanderern missbraucht werden. Der negativ konnotierte Terminus des »Scheinasylanten« stand für die ablehnende oder zumindest misstrauische Haltung gegenüber Personen, die Asyl beantragten.

Koser keine Aussage treffen. Dieser Frage gehen Czaika und Hobolth (2014) in ihrer oben genannten Studie zum »deflection effect« restriktiver Visa- und Asylpolitiken nach. 


\subsection{Visafreiheit als Tauschgut (seit den 2000ern)}

Der Versuch, Europa durch eine restriktive Visapolitik abzuschotten, ist nicht einfach beendet (vgl. Bigo/Guild 2005; Mau et al. 2012). Noch immer sind es vor allem andere reiche, demokratische Staaten, die in der EU von der Visumspflicht befreit wurden und es sind nach wie vor die armen und krisengeschüttelten Staaten des globalen Südens, die der Visumspflicht unterliegen. Diese Zweiteilung befördert einen "global mobility divide« (Mau et al. 2015), in dem Lebens- und Mobilitätschancen eng verbunden sind. Im Falle einer politischen Verfolgung etwa entscheiden die Reisemöglichkeiten mit darüber, ob es gelingt, andernorts Schutz zu suchen.

Doch fügen die EU-Mitgliedstaaten dieser restriktiven Politik neue Aspekte hinzu. So haben sich seit der ersten Festlegung der Liste von Staaten, deren BürgerInnen ein Visum benötigen (Phase zwei), einige interessante Veränderungen ergeben, die auf eine selektive Öffnung hinweisen.

Beispielsweise wurden die BürgerInnen der Staaten des Westbalkans sowie der Republik Moldau von der Pflicht befreit, ein Visum vor der An- und Einreise in die EU zu beantragen. Slowenien und Kroatien wurden bereits 2001 vor dem Hintergrund ihrer konkreteren Beitrittsperspektive (der Beitritt erfolgte 2004 und 2013) von der Visumspflicht entbunden. Serbien, Montenegro und Mazedonien folgten 2009. BürgerInnen aus Bosnien und Herzegowina dürfen seit Ende 2010 visafrei in die EU einreisen. Vorausgegangen war diesen Befreiungen jeweils zunächst die Einführung von Visaerleichterungen als »konkreter Schritt zur Abschaffung der Visumpflicht« (siehe beispielhaft Abkommen zwischen der Europäischen Gemeinschaft und der Republik Serbien zur Erleichterung der Visaerteilung, Amtsblatt der Europäischen Union L334/137, 19.12.2007).

Im Mai 2014 einigten sich die Mitgliedstaaten der Europäischen Union dann auf noch weitere 17 Herkunftsländer, deren StaatsbürgerInnen zukünftig ohne ein Visum für drei Monate in die EU und den Schengenraum einreisen dürfen. Diese zunächst großzügig wirkende Geste relativiert sich schnell, wenn man auf die Liste der neu befreiten Staaten schaut: Kiribati (ca. 102.000 Einwohner), Samoa (193.000), Tuvalu (10.000) und weitere 11 Insel-Kleinststaaten. Einzige Ausreißer hinsichtlich der Bevölkerungsgröße sind die Vereinten Arabischen Emirate (5,4 Mill) und Trinidad Tobago (1,3 Mill). Diese beiden Fälle sind unterschiedlich gelagert. Während hinter der Befreiung der Vereinten Arabischen Emirate wirtschaftliche Interessen durch Tourismus und Geschäftsreisende zu vermuten sind, löste die EU mit der Befreiung Trinidad Tobagos eine Reziprozitätserwartung ein, denn die meisten Europäer konnten bereits visafrei dort einreisen, wohingegen Trinidader und Tobagoer bis 2014 ein Visum für eine Europareise benötigten. 
Damit wurden im Laufe der Zeit insgesamt 29 Drittstaaten der Gruppe von zunächst 43 Ländern hinzugefügt, die schon seit 2001 visafrei reisen durften. Dreizehn der zunächst befreiten Staaten sind inzwischen der Europäischen Union und/oder dem Schengener Abkommen beigetreten und benötigen damit ohnehin kein Visum mehr (z.B. Kroatien, Polen, Schweiz). Für zwei Länder wurde die Visafreiheit zurückgenommen (Bolivien 2006, Ecuador 2003) ${ }^{15}$, was bedeutet, dass gegenwärtig 57 Staaten befreit sind. Dies entspricht etwa einem Drittel aller Drittstaaten der EU. Betrachtet man statt der Anzahl der Länder jedoch den Anteil, den diese Länder an der gesamten Weltbevölkerung ausmachen, so reduziert sich dieser Anteil auf 15,8\%. Nimmt man die Gruppe derer, die sich als EU-BürgerInnen ohnehin in Europa frei bewegen können, zusammen mit den Drittstaatlern, die ohne Visum einreisen dürfen, so hat sich diese Gruppe zwischen 2001 und 2013 kaum vergrößert. Der Anteil der Weltbevölkerung, der von einer Reisefreiheit in die EU profitiert, stieg damit nur von etwa $21 \%$ auf knapp $23 \%$ an (siehe Tabelle 1).

Tabelle 1: Reisefreiheit in die EU in Anteilen der Weltbevölkerung

\begin{tabular}{lll}
$\mathbf{2 0 0 1}$ & $\begin{array}{l}\text { Anzahl der } \\
\text { Länder }\end{array}$ & $\begin{array}{l}\text { Anteil an der } \\
\text { Weltbevölkerung }\end{array}$ \\
\hline EU & 15 & 6,14 \\
Befreite Drittstaaten & 43 & 14,95 \\
Summe & 58 & 21,09 \\
\hline $\mathbf{2 0 1 3 / 2 0 1 4}$ & & \\
\hline EU & 28 & 7,15 \\
Befreite Drittstaaten & 57 & 15,76 \\
Summe & 85 & 22,91
\end{tabular}

Quelle: Daten der Weltbank über Bevölkerungszahlen (siehe http://ata.worldbank.org/ indicator/SP.POP.TOTL, vom 9.12.2015)

15 Auch für den Südsudan wurde 2014 eine Visumpflicht eingeführt. Dies geht jedoch auf die Sezession des Landes zurück. BürgerInnen der alten Republik Sudan waren auch vor der Sezession bereits verpflichtet, ein Schengenvisum für touristische oder familiäre Besuche zu beantragen.

16 Die Anzahl der Länder bezieht sich auf das Jahr 2014. Die neuesten Daten über Bevölkerungspopulationen liegen bei der Weltbank jedoch bisher nur für das Jahr 2013 vor. 
Es liegt damit eine nominelle Öffnung vor, aber eigentlich keine Liberalisierung mit Blick auf die weltweiten Mobilitätschancen. Die Zulassungspolitik der EU gestaltet sich im Bereich des visafreien Reisens sehr selektiv (vgl. Mau et al. 2012).

Der Grund für die Visabefreiungen von bestimmten Staaten ist somit nicht in einer grundsätzlich liberaleren Haltung gegenüber Personenmobilität zu suchen. Stattdessen lassen sich die Fälle, in denen eine Visumspflicht aufgehoben wurde, außen- und wirtschaftspolitisch erklären. Das ist vor allem plausibel für die Vereinten Arabischen Emirate und Trinidad Tobago, von wo auch bei visafreiem Reisen keine größere Anzahl von MigrantInnen zu erwarten ist. Doch bei den abgeschlossenen oder noch laufenden politischen Verhandlungen über das visafreie Reisen von Bosniern, Serben oder Türken spielt(e) die Sorge um einen Anstieg der Einwanderungszahlen durchaus eine Rolle. ${ }^{17}$ Wie am einleitenden Beispiel der Türkei gesehen, muss sich diese Erwartung in Bezug auf Asylsuchende jedoch nicht unbedingt nur auf die BürgerInnen des betreffenden Landes beziehen. Es kann sich dabei auch um TransitmigrantInnen handeln.

Dadurch, dass der Türkei 2001 für alle europäischen Staaten eine Visumspflicht auferlegt wurde, ergab sich ein politisches Faustpfand. Mit diesem versucht die EU nun einen politischen Tausch zu initiieren. Die Kommission oder auch nationale RegierungsvertreterInnen verhandeln über Visaerleichterungen für türkische StaatsbürgerInnen unter der Auflage, dass die Türkei bereit ist, die Durchreise von TransitmigrantInnen aus dem arabischen Raum zu verhindern. Es wird sich zeigen, ob eine Delegation von Migrationskontrollaufgaben in Form einer verstärkten Grenzsicherung und der Bereitstellung von Flüchtlingslagern gelingen wird (vgl. Laube/Müller 2015). Die erwartete Gegenleistung der Türkei für die »Gabe« Visaerleichterungen befindet sich damit auf der operativen Ebene der Grenzpolitik.

Visaerleichterungen als Tauschgut in Verhandlungen mit den Nachbarstaaten einzusetzen, ist seit den 2000er Jahren in der EU eine anerkannte Praxis (vgl. Boniface/Wesseling 2008). Insbesondere den Staaten der östlichen Peripherie wurden Visaerleichterungen angeboten, sofern sie keine oder noch keine konkre-

17 In der Folge der Befreiungen von der Visumspflicht 2009 ist es in Deutschland zu einem sprunghaften Anstieg von Asylantragstellungen insbesondere aus Serbien und Mazedonien gekommen. Aus Serbien kamen im Jahr 2009 noch lediglich unter 900 AsylantragstellerInnen. 2012 waren es dann fast 13.000. Nahezu alle Asylanträge wurden jedoch als offensichtlich unbegründet beschieden. Siehe https:/www. bmi.bund.de/SharedDocs/Pressemitteilungen/DE/2014/01/asylzahlen_2013.html vom 9.12.2015. 
te Beitrittsperspektive hatten/haben. Teil solcher Verhandlungen sind in der Regel sogenannte Rücknahmeabkommen, die die Bereitschaft des Nachbarlandes untermauern, seine eigenen oder fremde Staatsbürger zurückzunehmen, sofern diese irregulär in die EU eingereist sind oder sich ohne gültige Dokumente aufhalten. Trauner und Kruse (2008) argumentieren, dass die Gewährung von Visaerleichterungen auch als positives Druckmittel für Reformen in den Nachbarländern genutzt wird. Visaerleichterungen beinhalten zumeist die Senkung der Kosten für ein Visum, die Reduzierung der vorzuweisenden Dokumente und Sicherheiten bei der Visabeantragung. Zu erwarten ist dadurch ein Anstieg der Bewilligungsquoten, auch wenn nach wie vor jeder Einzelfall geprüft wird. Visaerleichterungen können für Asylsuchende daher nur die Kosten des Versuchs, legal aus- und einzureisen senken, nicht aber verhindern, dass genau bei dieser Beantragung ein Verfolgerstaat auf die geplante Emigration aufmerksam wird.

Deutlich wird, dass hier die Visapolitik nicht mehr einfach als Mittel der Abschottung genutzt wird. Es wird eine selektive Öffnung gegenüber bestimmten Ländern in der EU-Peripherie vollzogen, indem für deren BürgerInnen die grenzüberschreitende Mobilität erleichtert wird (vgl. Mau et al. 2012). Diese Stoßrichtung ist Teil der offiziellen europäischen Außen- und Nachbarschaftspolitik (ENP) (vgl. Boniface/Wesseling 2008). Auch wenn im Hintergrund erneut Migrationskontrollinteressen stehen mögen, so gewinnt die Visapolitik doch eine neue Dimension: Die ausgewählte Zulassung und Liberalisierung wird als außenpolitisches Mittel eingesetzt, um eigene Interessen zu verfolgen. Es werden wieder mehr Länder von der Visumspflicht befreit, als dass Visumspflicht auferlegt wird. Damit ähnelt der Ansatz der dritten Phase der europäischen Visapolitik in gewisser Weise der ersten. Doch werden heute an das "Geschenk« der Öffnung für das visafreie Reisen klare Bedingungen und Gegenleistungen geknüpft (vgl. Vobruba 2000). Es besteht eine sehr konkrete Reziprozitätserwartung (vgl. Gouldner 1984 [1973]), die in bilateralen Abkommen, wie auch dem EU-Türkei-Abkommen, festgeschrieben wird. Auch die Westbalkan-Staaten hatten als Gegenleistung für die Befreiung Rücknahmeabkommen unterzeichnet (bereits in Kraft 2008), die es den EU-Mitgliedstaaten ermöglichen, Personen vereinfacht zurückzusenden, die keinen gültigen Aufenthalt (mehr) im Schengenraum genießen und nachweislich aus einem dieser Länder stammen oder über diese in die EU eingereist sind. ${ }^{18}$

18 Die Angaben zu den Rücknahmeabkommen sind dem Datensatz »Inventory of the Agreements linked to Readmission« entnommen. Zur Verfügung gestellt wird dieser auf der »Return Migration and Development Platform« des Robert-Schumann-Center 
Ein österreichischer Ministerialbeamter beschrieb 2009 diesen Tausch als neues Politikinstrument:

»Die Kompetenz für derartige Verschiebungen ${ }^{19}$ liegt seit dem Vertrag von Amsterdam bei der Kommission. [...] Das hat jetzt in den letzten [...] Jahren, [...] zu doch tendenziell gröberen Verschiebungen geführt; und zwar ist jetzt durch die Kommission eine weitere Methode dazwischengeschaltet worden. Ursprünglich war es ja: visumpflichtig/visumfrei; jetzt hat man, um da einen graduellen Prozess einzuleiten, das Instrument der Visumerleichterung erfunden. Das ist jetzt beschlossen worden für den West-Balkan, für Moldawien, für Russland, für die Ukraine. Dieses Instrument der Visumerleichterung ist sozusagen die Stufe 1, die gezündet worden ist hin zu einer möglichen zukünftigen Visumfreiheit. Und das führt uns jetzt auch ein bisschen schon zur Methode, wie man jetzt in diesem neuen Regime die Visumbefreiung angeht. [...] Man nimmt sie erst einmal in den Bestand her und gibt längere Fristen, geringere Gebühren, länger dauernde Visumzeiten und so weiter, das ist im Prinzip die Eigenheit der Visumerleichterungsabkommen, verlangt aber dann von den Zielstaaten mehrere Faktoren, die da sind: unbedingte Dokumentensicherheit, ein Rückübernahmeabkommen, bestimmte Informationsverpflichtungen über illegale Migration und - grob gesprochen - eine verbesserte polizeiliche Zusammenarbeit. Das heißt man spürt nicht immer nur flach 〉ist er jetzt visumspflichtig oder nicht?«, sondern wenn man von Visumpflicht auf Visumfreiheit geht, verlangt man ein umfangreiches $\mathrm{Pa}$ ket von Maßnahmen, damit gewährleistet ist, dass eine allfällige Visumfreiheit nicht mit einem Verlust von Sicherheit einhergeht.« (Interview Österreich, BMI, 2009, \#13)

Was der Interviewte als Sicherheit bezeichnet, bringt uns zurück zu der Frage, welche Implikationen diese Politik für Asylsuchende hat. Die Absicht der politischen AkteurInnen besteht darin, eine stufenweise Öffnung gegenüber den Nachbarn einzuleiten, um sicherzustellen, dass diese Nachbarstaaten - als Transitstaaten für Flüchtlinge - Kontrollaufgaben übernehmen (vgl. Laube/Müller 2015). Der territoriale Zugang über diese Staaten in die EU soll so zusätzlich kontrolliert werden, um auch den Zuzug von Asylsuchenden zu reduzieren (vgl. Guiraudon 2006; Lax 2008). Sofern es gelingt, Flüchtlinge gar nicht erst einreisen zu lassen, greifen auch noch keine rechtlichen Verpflichtungen für diese Personen. Für die befragten Ministerialbeamten stellte die Vermeidung rechtlicher

am Europäischen Universitätsinstitut (EUI), die Erhebungen macht das Team rund um Jean-Pierre Cassarino (vgl. Cassarino 2010 und http://rsc.eui.eu/RDP/).

19 Kommentar L.L.: Mit Verschiebungen sind hier Veränderungen in der gemeinsamen EU-Visapolitik gemeint, d.h. sowohl die Aushandlung von Visaerleichterungen als auch Aufhebungen der Visumspflicht. 
Verpflichtungen einen der Hauptgründe für den Einsatz von exterritorialen Migrationskontrollinstrumenten, wie der Visumspflicht, dar (ausführlicher dazu Laube 2013: 256-260).

Für Asylsuchende, die nach Westeuropa wollen, bedeutet dies, dass durch einen politischen Tausch in der Visapolitik bereits auf dem Weg in ihr Zielland Instanzen durch die polizeiliche Zusammenarbeit in Stellung gebracht werden, die ihre Weiterreise zu verhindern suchen. Nur durch die Umgehung der offiziellen Wege und Routen ist eine Anreise in ihre Zielländer noch möglich. Der Versuch, Sicherheit für das eigene Land herzustellen, wie dies der Ministerialbeamte anspricht, bedeutet für Asylsuchende, dass sie auf immer riskantere und oft lebensgefährliche Wege nach Europa abgedrängt werden.

\section{Reisefreiheit und Abschottung in der europäischen Peripherie}

In der politischen Debatte sowie der Forschungsliteratur über Asylpolitik war der Bereich Visa fast vollständig abwesend. Dabei haben sich in diesem Beitrag vielfältige Verknüpfungen zwischen diesen beiden Politikfeldern aufzeigen lassen, die letztlich Teile eines gemeinsamen Grenzregimes sind. Auch die gegenwärtige Diskussion über die Ankunft von vielen Hunderttausend MigrantInnen und Asylsuchenden in Europa thematisiert zwar die Abwesenheit legaler Zuwanderungsmöglichkeiten, kaum aber, dass sich mit einer liberaleren Visapolitik die Tragödien rund um das Mittelmeer deutlich verringern ließen. Schutzsuchende könnten offizielle Transportmittel und Routen nutzen und wären nicht auf die Dienstleistungen von Schleppern angewiesen. Ob und mittels welcher Aufenthaltstitel sie dann bleiben könnten, würde sich erst vor Ort entscheiden (wie de facto auch durch den Zusammenbruch des Dublin-/Schengensystems 2015). Die aufnehmenden Länder bleiben dann jedoch mit der Frage zurück, wie sie einmal angekommene Personen wieder abschieben können, sofern diese keine legale Bleibeperspektive erhalten, z.B. weil ihr Asylantrag abgelehnt wurde (vgl. Noll 1999). Um diesem Problem aus dem Weg zu gehen, haben sich die EU Staaten in den vergangenen Dekaden der migrationspolitischen Strategie der räumlichen Exterritorialisierung von Kontrollen bedient. Immer mehr Kontrollinstrumente greifen bereits vor der Ankunft von Schutzsuchenden an der eigentlichen Grenze in die Mobilität ein, zu allererst und am effektivsten die Visapolitik (vgl. Laube 2013; Czaika/Hobolth 2014).

Dass heute Visapolitik jedoch nicht mehr nur als abschottendes Instrument eingesetzt wird, sondern vielfach Gegenstand bilateraler Verhandlungen der EU 
mit den Staaten der erweiterten europäischen Nachbarschaft ist, wurde bisher in der Literatur unterschätzt. Gerade dort, wo Beitrittsperspektiven enden oder noch in weiter Ferne liegen, geht die EU dazu über, kleinere Offerten zu machen, in Form von Visaerleichterungen oder der Aufhebung der Visumspflicht. Gegenwärtig sind die Verhandlungspartner Staaten wie die Türkei, die Ukraine, Russland, Serbien oder Bosnien. Dabei handelt es sich um Länder, die oft zugleich als sichere Herkunftsländer gelten, sodass Asylanträge von deren eigenen BürgerInnen ohnehin negativ beschieden werden (so Serbien, Mazedonien, Bosnien). ${ }^{20}$

Die Visabefreiung eines Drittstaates markiert auch die Anerkennung, dass es sich um einen stabilen, demokratischen Staat handelt, aus dem keine größeren Auswanderungswellen zu erwarten sind (vgl. Mau/Brabandt 2011; Laube/ Heidler 2016). Der Staat und die meisten seiner BürgerInnen mögen von einer Befreiung von der Visumspflicht durch die EU profitieren. Doch ein solcher Wandel verändert auch die Bedingungen für Flüchtlinge aus diesen Ländern oder von Personen, die diese Länder als Transitland nutzen. Wenn diese Länder fortan als sichere Herkunftsländer wahrgenommen werden, kann sich dies auf die Anerkennungschancen von Asylsuchenden im Verfahren auswirken.

Doch systematische Studien über den Zusammenhang zwischen Visa- und Asylpolitik gibt es bisher nur vereinzelt (vgl. Trauner/Kruse 2008; Czaika/Hobolth 2014). Zumeist blicken diese Studien auf den Wandel der Antragszahlen durch eine Veränderung von Visapolitik (Befreiung/Einführung der Visumspflicht). Doch welchen Einfluss hat die Aufhebung der Visumspflicht auf die Chancen, ein Asylverfahren erfolgreich zu durchlaufen? Wie verändert sich die Sichtweise von MinisterialbeamtInnen und AsylentscheiderInnen auf Asylsuchende aus diesen Ländern? Dieser Beitrag konnte auf Basis von Experteninterviews erste Hinweise liefern, die diesen Zusammenhang erhellen. Doch liegt hier ein immenser Forschungsbedarf, der nicht länger übersehen werden darf.

20 Dies gilt nicht für Russland oder die Türkei, da von dort nach wie vor Asylanträge stammen, die im Falle von Kurden bzw. Tschetschenen auch eine Chance auf Bewilligung haben. Russland gehörte noch 2013 zu den 10 Ländern, aus denen in Deutschland die meisten Anträge eingegangen waren. Auch von Serben, Bosniern und Mazedoniern werden nach wie vor viele Anträge gestellt, doch diese werden inzwischen alle als offensichtlich unbegründet abgewiesen, seitdem diese Herkunftsländer den Status eines sicheren Drittstaates erhalten haben. Für eine knappe Übersicht der Antragszahlen und Herkunftsländer siehe https://www.bmi.bund.de/SharedDocs/Presse mitteilungen/DE/2014/01/asylzahlen_2013.html und http://www.bmi.bund.de/Shared Docs/Pressemitteilungen/DE/2015/01/asylzahlen_2014.html vom 9.12.2015. 
Die dritte Phase der europäischen Visapolitik wird dauerhaft Elemente der ersten beiden verbinden. Das politische Augenmerk liegt zwar momentan auf der Visumsfreiheit und dem politischen Tausch, der damit erreicht werden kann. Doch auch die Absicht, Europa vor einer wachsenden und unkontrollierten $\mathrm{Zu}$ wanderung zu schützen, bleibt aktuell. Nachdem sich viele der restriktiven Instrumente im Jahr 2015 als nicht krisensicher erwiesen haben, werden wir wohl Zeugen einer noch deutlicheren Akzentuierung der Abschottung und Zurückweisung von Flüchtlingen und MigrantInnen werden. Doch bezüglich der Beziehungsebenen (Staat/Staat, Staat/Bürger), auf denen Visapolitik funktioniert, hat sich die europäische Politik darauf zurückbesonnen, Visapolitik als außenpolitisches Instrument einzusetzen. Individuen können und werden sich den Zulassungsregeln entziehen, auch wenn der Preis dafür bisweilen sehr hoch ist. Die größere Bindungskraft versprechen sich die politischen AkteurInnen von der Visapolitik, wenn sie als politisches Gut in den Beziehungen zwischen der EU und ihren Nachbarländern eingesetzt wird. Denn die BürgerInnen aus Ländern wie der Türkei, Serbien oder Bosnien wünschen sich die Reisefreiheit in die EU ${ }^{21}$ und diese können ihre Regierungen für sie nur erwirken, wenn sie bereit sind, als Gatekeeper für die EU zu fungieren.

\section{Literatur}

Bigo, Didier/Guild, Elspeth (2005): »Policing at a Distance: Schengen Visa Policies«, in: Dies. (Hg.), Controlling Frontiers. Free Movement Into and Within Europe, Aldershot: Ashgate, S. 233-274.

Bogner, Alexander/Littig, Beate/Menz, Wolfgang (Hg.) (2005): Das Experteninterview. Theorie, Methode, Anwendung, Wiesbaden: VS Verlag für Sozialwissenschaften.

Boniface, Jérôme/Wesseling, Mara (2008): Visa Facilitation versus Tightening of Control: Key Aspects of the ENP, Brüssel: European Parliament.

21 Den Wunsch zum Beispiel der türkischen BürgerInnen nach einer Reisefreiheit in die EU bezieht u.a. EU-Kommissionspräsident Jean-Claude Juncker direkt in seine strategischen Überlegungen ein. Er sieht bei einem möglichen Scheitern des EU-TürkeiAbkommens den Staatspräsidenten Erdoğan in der schwierigen Lage, dies seinem Volk zu erklären. Doch dies sei dann »nicht mein Problem, das wird sein Problem sein«, siehe http://www.zeit.de/politik/ausland/2016-05/tuerkei-erdogan-visafreiheitjuncker-merkel, vom 12.5.2016. 
Cassarino, Jean-Pierre (Hg.) (2010): Unbalanced Reciprocities: Cooperation on Readmission in the Euro-Mediterranean Area, Washington: Middle East Institute.

Czaika, Mathias/Hobolth, Mogens (2014): Deflection into Irregularity? The (Un)intended Effects of Restrictive Asylum and Visa Policies, Oxford: International Migration Institute.

Eigmüller, Monika/Vobruba, Georg (Hg.) (2006): Grenzsoziologie. Die politische Strukturierung des Raumes, Wiesbaden: VS Verlag für Sozialwissenschaften.

Geiger, Martin/Pécoud, Antoine (Hg.) (2013): Disciplining the Transnational Mobility of People, Houndmills: Palgrave Macmillan.

Gouldner, Alvin W. (1984 [1973]): »Die Norm der Reziprozität. Eine vorläufige Formulierung«, in: Ders., Reziprozität und Autonomie. Ausgewählte Aufsätze, (=Suhrkamp Taschenbuch Wissenschaft, Band 304), Frankfurt am Main: Suhrkamp, S. 79-117.

Groenendijk, Kees/Guild, Elspeth/Minderhoud, Paul (Hg.) (2003): In Search of Europe's Borders, The Hague: Kluwer Law International.

Guild, Elspeth (2003): »The Border Abroad. Visas and Border Controls«, in: Groenendijk/Guild/Minderhoud, In Search of Europe's Borders, S. 87-104.

Guiraudon, Virginie (2003): »Before the EU Border: Remote Control of the >Huddled Masses«", in: Groenendijk/Guild/Minderhoud, In Search of Europe's Borders, S. 191-214.

- (2006): »Enlisting Third Parties in Border Control: A Comparative Study of its Causes and Consequences«, in: Marina Caparini/Otwin Marenin (Hg.), Border and Security Governance. Managing Borders in a Globalised World, Wien: LIT Verlag, S. 79-96.

Hess, Sabine/Kasparek, Bernd (Hg.) (2010): Grenzregime. Diskurse, Praktiken, Institutionen in Europa, Berlin: Assoziation A.

International Air Transport Association (2014): Travel Information Manual, Amsterdam: IATA Netherlands Data Publications.

Koser, Khalid (2000): »Asylum Policies, Trafficking and Vulnerability«, in: International Migration Special Issue 2000(1), S. 91-111.

Laube, Lena (2013): Grenzkontrollen jenseits nationaler Territorien. Die Steuerung globaler Mobilität durch liberale Staaten, Frankfurt a.M.: Campus Verlag.

Laube, Lena/Heidler, Richard (2016): »Democratic Centre, Autocratic Isolates: The Changing Field of International Visa Policies«, in: Journal of Ethnic and Migration Studies (DOI: 10.1080/1369183X.2016.1176906, 13 May 2016). 
Laube, Lena/Müller, Andreas (2015): »Warum die Kontrolle abgeben? Die Delegation von Migrationskontrolle aus der Prinzipal-Agent-Perspektive«, in: Berliner Journal für Soziologie 25(3), S. 255-281.

Lax, Violeta Moreno (2008): »Must EU Borders have Doors for Refugees? On the Compatibility of Schengen Visas and Carriers' Sanctions with EU Member States' Obligations to Provide International Protection to Refugees «, in: European Journal of Migration and Law 10(3), S. 315-364.

Luedtke, Adam/Byrd, Douglas G./Alexander, Kristian P. (2010): »The Politics of Visa«, in: The Whitehead Journal of Diplomacy and International Relations 11(1), S. 147-160.

Mau, Steffen/Brabandt, Heike (2011): »Visumpolitik und die Regulierung globaler Mobilität«, in: Zeitschrift für Soziologie 40(1), S. 3-23.

Mau, Steffen/Brabandt, Heike/Laube, Lena/Roos, Christof (2012): Liberal States and Freedom of Movement. Selective Borders, Unequal Mobility, Basingstoke: Palgrave Macmillan.

Mau, Steffen/Gülzau, Fabian/Laube, Lena/Zaun, Natascha (2015): »The Global Mobility Divide: How Visa Policies have Evolved Over Time«, in: Journal of Ethnic and Migration Studies 41(8), S. 1-22.

Neumayer, Eric (2004): »Asylum Destination Choice: What Makes Some West European Countries More Attractive Than Others?«, in: European Union Politics 5(2), S. 155-180.

Noll, Gregor (1999): Rejected Asylum Seekers: the Problem of Return, UNHCR, New Issues in Refugee Research, Working Paper No. 4.

- (2000): Negotiating Asylum. The EU Acquis, Extraterritorial Protection and the Common Market of Deflection, The Hague: Martin Nijhoff.

Parusel, Bernd/Schneider, Jan (2012): Visumpolitik als Migrationskanal, Bundesamt für Migration und Flüchtlinge, Working Paper der nationalen Kontaktstelle des EMN und der Forschungsgruppe des Bundeamtes, Nürnberg.

Thielemann, Eiko (2006): »The Effectiveness of Governments' Attempts to Control Unwanted Migration«, in: Craig Parsons/Timothy Smeeding (Hg.), Immigration and the Transformation of Europe, Cambridge: Cambridge UP, S. 444-474.

Trauner, Florian/Kruse, Imke (2008): »EC Visa Facilitation and Readmission Agreements: A New Standard EU Foreign Policy Tool?«, in: European Journal of Migration and Law 10(4), S. 411-438.

Vobruba, Georg (2000): »Integration und Expansion. Zur Erweiterung der Interessensphäre Europas«, in: Blätter für deutsche und internationale Politik 3, S. 335-340. 
Witzel, Andreas/Reiter, Herwig (2012): The Problem-centred Interview. Principles and Practice, London: Sage.

\section{Internetquellen}

Bielicki, Jan/Seeling, Luisa (2015): »Mehr als eine Million Flüchtlinge sollen bis Ende 2015 in Deutschland ankommen«, in: Süddeutsche Zeitung, http://www.sueddeutsche.de/politik/deutschland-mehr-als-eine-millionfluechtlinge-1.2755884 vom 1.12.2015.

Bundesministerium des Innern: Humanitäre Aufnahmeprogramme des Bundes, http://www.bmi.bund.de/DE/Themen/Migration-Integration/Asyl-Fluechtlingsschutz/Humanitaere-aufnahmeprogramme/humanitaere-aufnahmepro gramme_node.html vom 1.12.2015.

Bundesministerium des Innern (2014): »Anzahl der Asylbewerber im Jahr 2013: Höchster Stand seit 14 Jahren«, https://www.bmi.bund.de/SharedDocs/ Pressemitteilungen/DE/2014/01/asylzahlen_2013.html vom 1.12.2015.

Bundesministerium des Innern (2015): »202.834 Asylanträge im Jahr 2014«, http://www.bmi.bund.de/SharedDocs/Pressemitteilungen/DE/2015/01/asylza hlen_2014.html vom 1.12.2015.

Deutsche Bundesregierung (2016): Entwurf eines Gesetzes zur Bestimmung von Algerien, Marokko und Tunesien als sichere Herkunftsstaaten, https://www.proasyl.de/wp-content/uploads/2016/02/160129_Referentenentwurf_Maghrebstaaten_sichere_HKS.pdf vom 17.6.2016.

European Commission (2016): „Overview of Schengen Visa Statistics«, http://ec.europa.eu/dgs/home-affairs/what-we-do/policies/borders-and-visas/ visa-policy/docs/overview_of_schengen_visa_statistics_en.pdf vom 28.01.2016.

European University Institute: Return Migration and Development Platform, http://rsc.eui.eu/RDP/ vom 28.01.2016.

Laube, Lena et al.: Visa Network Data, www.fiw.uni-bonn.de/demokratieforschung/personen/laube/visanetworkdata vom 28.01.2016.

Luther, Carsten (2016): »Fressen was Erdoğan auftischt?«, http://www.zeit. de/politik/ausland/2016-05/tuerkei-erdogan-visafreiheit-juncker-merkel vom 12.5.2016.

Rinke, Andreas (2015): »Germany launches media drive to counter refugee rumors«, http://www.reuters.com/article/us-europe-migrants-germany-embas sies-idUSKCNORE1JM20150914 vom 1.12.2015. 
UNHCR (2015): »Mittelmeer: Rekordzahl an Flüchtlingen und MigrantInnen«, http://www.unhcr.de/home/artikel/435da63ac57f3eaee63d95d2e4f7eb9b/mitt elmeer-rekordzahl-von-fluechtlingen-und-migranten.html vom 1.12.2015.

Weltbank: Data on Population, total, http://data.worldbank.org/indicator/SP. POP.TOTL vom 1.12.2015.

Zeit Online (2015): »Kampagne gegen das Ziel Deutschland«, http://www.zeit.de/politik/deutschland/2015-08/fluechtlinge-asyl-albanienserbien-bundesamt vom 1.12.2015.

Zeit Online (2015): »Knapp 356.000 unbearbeitete Asylanträge im Bamf«, http://www.zeit.de/politik/deutschland/2015-12/asyl-bamf-verwaltung-ueber forderung-asylantraege vom 10.12.2015. 


\section{Über Asylanträge entscheiden: Zur Praxis behördlichen Handelns}





\section{„Ohne 'ne ordentliche Anhörung kann ich keine ordentliche Entscheidung machen ... «}

Zur Organisation von Anhörungen

in deutschen und schwedischen Asylbehörden ${ }^{1}$

STEPHANIE SCHNEIDER UND KRISTINA WOTTRICH

»[D]ie Hauptarbeit oder die mit dem mit dem meisten wo wir die meiste Kompetenz reinstecken müssen aus meiner Sicht ist die Anhörung (.) weil sie ist (.) für mich das Herzstück was ich dann dann nachher entscheide ist wichtig keine Frage (.) aber ohne ne ordentliche Anhörung (2) kann ich keine ordentliche Entscheidung machen

(.) ja das eine bedingt das andere« (TRAINER* IN PIETSCH, Z. 818-824)

\section{Einleitung}

Seit Jahrzehnten arbeitet die Europäische Union auf die Schaffung eines Gemeinsamen Europäischen Asylsystems (GEAS) hin. Wie insbesondere die Ereignisse im Jahr 2015 zeigen, kann von dessen praktischer Realisierung keine Rede sein. Im Gegenteil scheinen nationale Sonderwege verbreiteter denn je.

1 Die diesem Beitrag zugrundeliegende Forschung wurde ermöglicht durch die Förderung der DFG (FOR 1539, Teilprojekt 02). Für hilfreiche Rückmeldungen zu früheren Versionen dieses Papiers danken wir Christian Lahusen, Karin Schittenhelm, Jacqueline Klesse und Carolin Nieswandt. 
Während angesichts divergierender Anerkennungsquoten eines der zentralen Ziele des GEAS, nämlich dass gleiche Fälle gleich behandelt werden (siehe Richtlinie 2013/32/EU), lange Zeit im Mittelpunkt der Diskussionen stand, streiten sich die Mitgliedsstaaten nun vornehmlich darum, wer überhaupt zur Aufnahme und Unterbringung von Geflüchteten bereit ist. Die scheinbare Eindeutigkeit administrativ hergestellter Entscheidungen über berechtigte oder unberechtigte Anträge auf internationalen Schutz bleibt dabei weitgehend unhinterfragt. Vielmehr wird im öffentlichen Diskurs auf schnellere Verfahren sowie die konsequentere Abschiebung derer gedrängt, bei denen - über die Definition vermeintlich sicherer Dritt- und Herkunftsstaaten - ein negativer Verfahrensausgang bereits im Vorhinein festzustehen scheint.

Die europäischen Institutionen verfolgen die Ziele einer Angleichung und Effizienzsteigerung nationaler Verwaltungspraktiken auf mehreren Ebenen und mit unterschiedlichen Instrumenten. Ein Instrument wird uns in diesem Beitrag besonders interessieren, denn in einigen Mitgliedsstaaten wird neben asylrechtlichen Verschärfungen auch mit einer Verstärkung des Personals in den Behörden auf gestiegene Antragszahlen reagiert. Bei der Einarbeitung dieses neuen Personals wird vermehrt auf ein europäisches Schulungsprogramm für Asylsachbearbeiter*innen zurückgegriffen: das seit 2012 beim Europäischen Unterstützungsbüro für Asylfragen (EASO) angesiedelte EASO Training Curriculum (im Folgenden kurz: ETC) (vgl. EASO 2016). Europäische Schulungsmaßnahmen werden konzipiert, um Verwaltungspraktiken auf der Organisations- und Personalebene durch die Einführung >neuer« Deutungen und Wissensbestände und im Sinne eines einheitlicheren Vorgehens zu verändern. Sie bieten sich einer soziologischen Forschungsagenda somit in besonderer Weise an, um zu untersuchen, wie Verwaltungsbehörden auf der Ebene des Vollzugs mit Veränderungs- und Harmonisierungsbestrebungen umgehen.

Aus der verwaltungssoziologischen Forschung wissen wir, dass die in Schulungen enthaltenen Leitbilder und Wissensbestände bei der Umsetzung den jeweiligen lokalen Arbeitsanforderungen und -routinen angepasst, re-interpretiert und mit bereits vorhandenem Wissen in Verbindung gebracht werden (vgl. Evans 2011; Feldman/Pentland 2003). Auf welche Weisen diese Aneignung, ReInterpretation und Verknüpfung konkret vonstattengehen kann, möchten wir im vorliegenden Beitrag in behördenvergleichender Perspektive diskutieren. Dabei nehmen wir an, dass der Umgang mit neuen Deutungen und Wissensbeständen nicht als Übernahme oder Abwehr zu verstehen ist, denn in der Erledigung ihres Arbeitsauftrages haben die Behördenmitarbeiter*innen konkrete Probleme innerhalb vordefinierter Arbeitsabläufe und Organisationsstrukturen zu lösen. Der Umgang mit den Schulungsinhalten ist daher von Konflikten und Aushandlun- 
gen, vom Ausprobieren und Verwerfen geprägt. Die neuen Deutungen und Wissensbestände bieten den Mitarbeiter*innen darin potenzielle Handlungsanweisungen und Rechtfertigungsmöglichkeiten zugleich an. Der vorgeschlagene Behördenvergleich ist besonders dafür geeignet, herauszustellen, wie diese aktive und kreative Aneignung von organisationalen Bedingungen mit geprägt ist.

Bei unseren Analysen stützen wir uns auf Daten, die im Rahmen eines qualitativ angelegten Forschungsprojekts zur Europäisierung des Asylverwaltungsfeldes $^{2}$ im deutschen Bundesamt für Migration und Flüchtlinge (BAMF) und in der schwedischen Migration Agency (Schwedisch: Migrationsverket, im Folgenden kurz SMA) erhoben wurden. Mit dem BAMF und der SMA nahmen wir Behörden in den Blick, die über lange asylrechtliche Traditionen und Routinen in der Durchführung von Asylverfahren verfügen und im europäischen Vergleich eine hohe Anzahl erstinstanzlicher Asylentscheidungen treffen. ${ }^{3}$ Im Projekt ging es u.a. darum, die Etablierung und Umsetzung des ETC zu untersuchen. Zu diesem Zweck wurden 2013/2014 in beiden Behörden Interviews mit Trainer*innen dieses Schulungsprogramms und mit Asylsachbearbeiter*innen durchgeführt. ${ }^{4}$ Wei-

2 »Auf dem Weg zu einem europäischen Asylverwaltungsfeld? Transnationale Verwaltungskooperation zwischen Asylbehörden« (Projektleitung: Christian Lahusen und Karin Schittenhelm, Universität Siegen), Teilprojekt 02 der DFG-Forschergruppe »Europäische Vergesellschaftungsprozesse« (FOR 1539).

3 Nach EUROSTAT-Angaben wurden in Deutschland im Jahr 201497.415 erstinstanzliche Asylentscheidungen getroffen, in Frankreich 68.500 und in Schweden 40.015. Dahinter folgte Italien mit 35.180 erstinstanzlichen Entscheidungen (EUROSTAT 02.03.16). Für das Jahr 2015 liegen bei der Verschriftlichung dieses Beitrags die Zahlen noch nicht für alle Mitgliedsstaaten vor. Für Deutschland verzeichnet EUROSTAT 249.280, für Italien 71.345 und für Schweden 44.590 erstinstanzliche Entscheidungen (ebd.).

4 Die leitfadengestützten Interviews fanden in den Räumlichkeiten der Behörden, meist in den jeweiligen Büros der Mitarbeiter*innen, statt. Insgesamt wurden im BAMF 15, in der SMA 26 Personen befragt. In Schweden wurden die Interviews mit Expert*innen sowie Trainer*innen vorwiegend auf Englisch, diejenigen mit Mitarbeiter*innen auf der street-level-Ebene auf Schwedisch geführt. Für den vorliegenden Beitrag wurden die Auszüge aus den schwedischsprachigen Interviews ins Englische übersetzt. Bei der Datenauswertung nutzen wir eine Kombination von dokumentarischer Methode (Bohnsack 2010; Nohl 2012) und Verfahren der Grounded Theory (Strauss/Corbin 1990), u.a. auch im Rahmen der Situationsanalyse (Clarke 2012). Dabei sind wir v.a. an einem verstehenden Nachvollzug der Zusammenhänge zwischen 
tere im Rahmen der Teilstudien erhobene Daten umfassten die beobachtende Teilnahme an ETC- und anderen Schulungsmaßnahmen sowie kürzere Beobachtungen der Arbeit der Sachbearbeiter*innen in unterschiedlichen Außenstellen. Während der Forschungsarbeiten hatten wir Zugang zur E-Learning-Plattform des EASO und haben dort einige der Schulungsmodule selbst durchlaufen.

Für die Zwecke dieses Beitrags stützen wir uns auf eine selektive Auswertung von Schulungsmaterialien, Beobachtungsnotizen und nach thematischen Gesichtspunkten ausgewählten Interviewpassagen, die einen spezifischen Ausschnitt des Verfahrens zum Gegenstand haben: die sogenannte >Anhörung〈 der von den Antragsteller*innen vorgebrachten Gründe für das Asylgesuch. Bei der Anhörung treffen Sachbearbeitende und Asylsuchende aufeinander und es werden wichtige Grundlagen für die Entscheidungsfindung geschaffen. Wer in einer Asylbehörde über Anträge (mit-)entscheidet, muss lernen, Anhörungen so durchzuführen, dass sie dem letztlichen Ziel - einer eindeutigen Entscheidung dienen, verfahrensrechtlichen Standards genügen und gleichzeitig lokalen Arbeitsanforderungen angemessen sind. Aufgrund der Unwägbarkeiten und der Komplexität sozialer Interaktionen lassen sich Anhörungen nicht ohne weiteres standardisieren und steuern. Gleichzeitig ist die Anhörung ein Verfahrensmoment, das in seinen zentralen Herausforderungen allen europäischen Asylverwaltungsbehörden gemeinsam ist. Jenseits von Unterschieden auf der Makroebene nationaler Asylsysteme sollte sich an diesem Beispiel daher besonders gut untersuchen lassen, wie die dort vermittelten Inhalte auf dem >street-level (Lipsky 2010) praktisch relevant (gemacht) werden. ${ }^{5}$

Der Beitrag ist folgendermaßen strukturiert. Zunächst werden wir auf die Rolle von gemeinsamen Schulungsprogrammen bei der Etablierung des GEAS eingehen und die Relevanz der organisationalen Bedingungen der Sachbearbeitung für eine Analyse des Umsetzungsprozesses herausstellen (Abschnitt 2). Im Hauptteil des Beitrags zeigen wir zunächst die zentrale Bedeutung der Anhörung für die Entscheidungsfindung im Asylverfahren auf und stellen das EASOModul 〉Gesprächsführungstechniken`vor (3.1). Anschließend werden beide Be-

kollektiven Praktiken der Sachbearbeitung und den in den Behörden vorhandenen Wissensbeständen und handlungsleitenden Orientierungen interessiert.

5 Zum Zeitpunkt unserer Datenerhebung wurde das Modul im BAMF zwar noch nicht offiziell umgesetzt, die snationalen< Schulungen orientierten sich aber bereits an den im ETC empfohlenen Methoden (Feldnotizen BAMF, Schulungen 1 und 2; Gespräche mit Dozent*innen und Trainer*innen). Einige der Befragten waren zudem bereits zum/zur Trainer*in für dieses Modul ausgebildet worden und vermittelten ihr Wissen eher informell an Kolleg*innen in ihren Außenstellen weiter. 
hörden mit Blick auf wichtige Unterschiede in der Organisation von Anhörungen charakterisiert. Dabei werden wir zeigen, dass sich die Praktiken der Sachbearbeitung v.a. dahingehend differenzieren lassen, dass diese in der SMA arbeitsteiliger organisiert sind und vielfältige Formen mündlichen Austauschs beinhalten (3.2), während die Sachbearbeitung im BAMF stärker individualisiert und schriftbasiert ist (3.3). Diesen Befund diskutieren wir schließlich mit Blick darauf, wie organisationale Gegebenheiten den Umgang der Sachbearbeiter*innen mit neuen Deutungen und Wissensbeständen mit prägen und auf welche Weisen diese Eingang in konkrete Handlungsroutinen und/oder Rechtfertigungen derselben finden (3.4). Der Beitrag schließt mit einem Ausblick (Abschnitt 4).

\section{Asylverwaltungsbehörden zwischen europäischer Regulierung und lokalen Routinen}

Im Bereich des Asyls erlangte die Kooperation zwischen europäischen Behörden v.a. mit der Abschaffung der Binnengrenzkontrollen und den Schengener Übereinkommen zunehmende Bedeutung. Im Maastrichter Vertrag von 1992 wurde das Thema erstmals als \Angelegenheit gemeinsamen Interesses` identifiziert. 1999 folgte der Beschluss der europäischen Staats- und Regierungschefs in Tampere zur Einführung eines Gemeinsamen Europäischen Asylsystems (GEAS), welcher die Harmonisierung der Asylverfahren und einen bindenden Rechtsrahmen vorsah. ${ }^{6}$ Mit dem Haager Programm wurden 2004 zunächst Richtlinien und Verordnungen über geltende Mindeststandards ausgehandelt, welche in einem zweiten Harmonisierungsversuch 2013 überarbeitet wurden und nun nicht mehr Mindestnormen, sondern gemeinsame, teils höhere, Standards vorsehen. ${ }^{7}$

Von einer Konvergenz europäischer Asylverfahren lässt sich jedoch nicht sprechen; nach wie vor unterscheiden sich Verfahrensweisen wie Anerkennungsquoten, selbst in Bezug auf einzelne Herkunftsländer, erheblich (vgl. Toshkov/Haan 2013; Parusel 2015a). Neben der unterschiedlichen Auslegung von

6 Zur Entstehung des GEAS s. u.a. Bendel (2013: 11ff.); Guild (2006); Lambert (2009); Kaunert/Léonard (2012); Ippolito/Velluti (2011).

7 Gegenwärtig besteht das GEAS im Wesentlichen aus drei Richtlinien, namentlich der Aufnahmerichtlinie 2013/33/EU, der Anerkennungsrichtlinie 2011/95/EU, der Asylverfahrensrichtlinie 2013/32/EU und zwei Verordnungen: der Dublin-III-Verordnung (EU) 604/2013 sowie der EURODAC-Verordnung (EU) 603/2013. 
Rechtsbegriffen, wie jenen der >inländischen Fluchtalternative` (vgl. Eaton 2013) oder der >bestimmten sozialen Gruppe` (vgl. Crawley/Lester 2004; Spijkerboer 2013), stützen sich die Behörden auf unterschiedliche Quellen für die Bewertung der Lage in den Herkunftsländern (vgl. Rosset/Maia Liodden 2015). Zudem bestehen neben und unterhalb des europäischen Rechts nationale (verfahrens-)rechtliche Besonderheiten weiter (vgl. Staffans 2008). Ebenso unterscheidet sich die praktische Arbeit der Behörden je nach institutionellem und organisationalem Aufbau der Asylsysteme und der jeweiligen Staats- und Rechtstraditionen. Da eine freie Wahl des Landes, in dem um Asyl ersucht wird, immer noch weitgehend verunmöglicht wird bzw. werden soll (vgl. Brekke/Brochmann 2015), sind solche Unterschiede, mit ihren für Asylsuchende erheblichen Konsequenzen, von besonderer Brisanz.

Im Kontext der geforderten stärkeren Angleichung behördlicher Praktiken wurde neben legislativen Maßnahmen im Rahmen des GEAS auch auf >weiche< Maßnahmen transnationaler Verwaltungskooperation gesetzt. In den letzten Jahren gewinnt das EASO bei der Koordinierung und Institutionalisierung solcher Formen der Zusammenarbeit und der gegenseitigen Unterstützung zwischen Asylbehörden an Relevanz. Ein zentrales Element stellt hierbei das EASO Training Curriculum für Asylsachbearbeiter*innen dar (vgl. EASO 2014a). Mit der Neufassung der Asylverfahrensrichtlinie (siehe Artikel 4, Absätze 1, 3 und 4 der Richtlinie 2013/32/EU) werden Schulungen für Asylsachbearbeiter*innen vorgeschrieben und es wird explizit auf die Angebote des EASO verwiesen (vgl. Verordnung 439/2010/EU). ${ }^{8}$ Mit dem ETC wird den Behörden ein fertig ausge-

8 Seit 2012 sind mit dem EASO Training Curriculum europaweit insgesamt $8.257 \mathrm{Be}$ hördenmitarbeiter*innen geschult worden (siehe https://easo.europa.eu/about-us/tasksof-easo/training-quality/training/), wobei sich die Umsetzung absolut betrachtet noch stark unterscheidet (vgl. EASO 2015a: 8). Sowohl in Schweden als auch in Deutschland kam es während unserer Feldforschungen 2013 und 2014 zu größeren Rekrutierungswellen und einem damit einhergehenden gestiegenen Schulungsbedarf. 2015 und 2016 setzte sich diese Entwicklung in noch erheblich gesteigerter Form fort. In Schweden konnte ca. vier Jahre früher (2009/2010, ab 2012/2013 sodann systematisch) mit den Schulungen begonnen werden, unter anderem da die Migration Agency die englischsprachigen Module nicht, wie das BAMF, hat übersetzen lassen. Mittlerweile sind die EASO-Schulungsmodule in beiden Behörden systematischer Bestandteil der amtsinternen Einführungsprogramme für neue Angestellte. Dem EASO >learning path entsprechend werden bislang v.a. die Kernmodule umgesetzt (vgl. EASO 2016). Während in Schweden im Jahr 2015 aufgrund des hohen Personalbedarfs die 
arbeitetes, modulares und aus Behördensicht vergleichsweise effizientes Instrument der Mitarbeiterschulung zur Verfügung gestellt. ${ }^{9}$ Mit der Entwicklung besonders entschlackter >Einsteigerkurse< soll in Zukunft auch die noch raschere Einarbeitung in Phasen höherer >case-loads` ermöglicht werden (vgl. EASO 2015b). Entwickelt werden die Schulungsinhalte im Rahmen transnationaler Verwaltungskooperation durch sogenannte >Modulexperten`, die zumeist aus den Mitarbeitenden der nationalen Behörden rekrutiert werden. Daneben sind Akteure aus der Wissenschaft (z.B. aus den Bereichen Jura oder Didaktik) und externe Referenzgruppen (internationale Organisationen, Akteure der Zivilgesellschaft, Europäische Kommission u.a.) an der Modulentwicklung beteiligt. Auf einer recht abstrakten Ebene vermitteln die Module zum einen Normen und Werte, an denen sich Asylsachbearbeiter*innen bei der Fallbearbeitung orientieren sollen. Je nach Modul sind diese nicht unbedingt spezifisch reuropäisch<, sondern können auch Teil »weltkultureller Skripts« (Meyer 2005) sein, indem bspw. auf allgemeine, wissenschaftlich begründete Standards der Fallbearbeitung oder auf menschen- und völkerrechtliche Verpflichtungen verwiesen wird, die für die Vertreter*innen europäischer Staaten verbindlich gelten sollen. Auf einer konkreteren inhaltlichen und praktischen Ebene stellen die Module zum anderen ein hybrides Gebilde dar, welches Elemente aus unterschiedlichen Rechts- und Verwaltungstraditionen nach dem Prinzip der Bricolage (vgl. Weick 1998) kombiniert und in konkrete Handlungsanweisungen für die Sachbearbeitung übersetzt. Versatzstücke aus unterschiedlichen Kontexten werden dabei weitgehend intakt gelassen, die Innovation besteht in ihrer Kombination und Systematisierung. Mit der Umsetzung werden sie wiederum Teil des behördlichen Repertoires, mit dem auf veränderte Umweltbedingungen reagiert werden kann. Hier können sie, durchaus flexibel und selektiv, in bestehende Verwaltungsroutinen eingespeist werden. Die Konzeption der Schulungen stellt insofern bereits eine Antwort auf das Problem fehlender Harmonisierung des mitgliedsstaatlichen Verwaltungshandelns dar.

Die EASO-Schulungsmaßnahmen zielen direkt auf die an den >front-lines der Behörden tätigen Behördenmitarbeiter*innen. Die europäische Ebene enthält sich allerdings einer Einwirkung auf die Mitgliedschaften der Organisationen

Trainingsaktivitäten eingeschränkt wurden (ebd.: 243f.), wurden sie im BAMF ausgeweitet (ebd.: 129f.).

9 Im Rahmen von >blended learning wird durch ein vorgeschaltetes E-LearningProgramm mit praktischen Übungen und theoretischen Reflexionen die Zeit für die anschließenden >face-to-face〈-Schulungen im Vergleich zu herkömmlichen Schulungsmaßnahmen reduziert. 
und überlässt die Entscheidung darüber, wer eigentlich befugt sein sollte, Asylentscheidungen zu treffen, den Mitgliedsstaaten. Es gilt die implizite Annahme, dass über das Instrument der Schulungen prinzipiell jede/r Bedienstete in die Lage versetzt werden könne, den geltenden Standards entsprechend über Asylanträge zu entscheiden, unabhängig davon, ob es sich in den jeweiligen nationalen Behörden bspw. um Polizeibeamte, Verwaltungsangestellte, Juristen oder Ethnologen handelt. So wird eine allgemeine Deutung >professioneller Asylsachbearbeitung installiert, die von beruflichen und behördlichen Sozialisationsprozessen ebenso abstrahiert wie von lokalen organisationalen Rahmen- und Arbeitsbedingungen.

Bei der praktischen Umsetzung der Schulungen sind diese jedoch von zentraler Bedeutung. Beiträge in der Tradition der >street-level bureaucracy` (vgl. Lipsky 2010; Brodkin 2011, 2008) haben für unterschiedliche Verwaltungsbehörden herausgearbeitet, dass Politik und auch Recht erst im Moment ihrer Umsetzung durch Mitarbeitende an den >front-lines` öffentlicher Verwaltungen wirksam werden und dabei unter Umständen verändert, teils erst `gemacht werden. Beim Umgang mit konkreten, praktischen Problemen in der täglichen Arbeit entwickeln Mitarbeiter*innen Handlungsroutinen, die formalen Organisationszielen entgegenstehen können. Das Verhältnis von gewandelten externen Vorgaben und Praktiken auf dem street-level ist allerdings ein - zumindest für den Bereich der Asylverwaltung - noch weitgehend ungeklärtes. Das Interesse der street-level-Theorie galt meist dem individuellen Umgang mit strukturellen Dilemmata des täglichen Arbeitens mit Klienten. Die Ebene der Organisation und insbesondere die Rolle des Managements wurde dabei weitgehend außer Acht gelassen (vgl. Bannink/Six/van Wijk 2015; Hupe/van Kooten 2015). Diese sind aber von besonderer Bedeutung, wenn es um das gezielte Einspeisen neuer Wissensbestände und Deutungen geht. Zudem wurde der Fokus oftmals auf Routinen und damit implizit auf Kontinuität gelegt (vgl. Miettinen/Virkkunnen 2005). Schulungen und Fortbildungen zielen nun gerade auf eine Bewusstmachung und Veränderung von Routinen des Arbeitshandelns, die als nicht mehr angemessen wahrgenommen werden (ebd.: 441f.).

Inwieweit (individuelle wie organisationale) Routinen allerdings tatsächlich zum Gegenstand von Reflexion werden können, ist eine empirisch zu klärende Frage. Wenn wir davon ausgehen, dass die Sozialisation vor Ort, das >trainingon-the-job< prägender ist als vereinzelte Schulungen (vgl. Lipsky 2010: 200), so müssen sowohl die Arbeitsbedingungen, Arbeitsteilungen und Kontrollstrukturen als auch die in den Behörden zirkulierenden Deutungen und Narrationen über angemessene >ways of doing things « in den Blick genommen werden (vgl. Feldman/Pentland 2003; Barley/Kunda 2001). Länder- bzw. behördenverglei- 
chende Studien zum praktischen Verwaltungshandeln und zu beruflichen Sozialisationsprozessen in Asylbehörden sind bislang jedoch rar gesät (siehe aber Probst 2012; Jubany 2011; Wettergren 2013). Hier möchte der vorliegende Aufsatz einen Beitrag leisten. Es geht uns dabei nicht um eine Bewertung der Umsetzung der EASO-Trainings, sondern um eine Analyse der organisationalen Bedingungen, auf die die Schulungsmaßnahmen in beiden Behörden treffen.

Aus einer wissenssoziologischen Perspektive interessiert uns dabei der $\mathrm{Zu}-$ sammenhang von explizit vermittelten Handlungsanweisungen und den eher impliziten Deutungen und Orientierungen, wie sie in den Behörden im täglichen Arbeiten relevant werden. Wir lassen also sowohl die Unterschiede auf der Makroebene staatlicher Asylsysteme als auch jene auf der Individualebene einzelner Sachbearbeiter*innen außer Acht und nehmen die Behörden in erster Linie als Organisationen vergleichend in den Blick. Dabei folgen wir der Annahme, dass das im Interview thematisierte oder in Schulungsmaterialien dokumentierte Wissen das Ergebnis situierter, kollektiver Handlungspraktiken ist und sich - nicht nur, aber eben auch - als organisationales Wissen untersuchen lässt (Liebig 2007).

\section{Anhören und entscheiden}

\subsection{Die Bedeutung von Anhörungen im Asylverfahren}

Zentrale Aufgabe von Asylbehörden ist es, legitime und rational begründete Entscheidungen über Schutzgewährung zu treffen und zu zertifizieren. Das jeder Entscheidung letztlich innewohnende Element von Willkür muss unerkannt bleiben, damit der Glaube an die Legitimität bürokratischer Akte der Zertifizierung aufrechterhalten werden kann (vgl. Bourdieu 1987: 844; 1994). Im Kontext der »chronisch knappe[n] Urteilskraft der Entscheider« in Asylverfahren (Scheffer 2003: 425) stellt die Anhörung der Antragsteller*innen die zentrale Grundlage für die Ermittlung des Sachverhalts, die Glaubhaftigkeitsprüfung und schließlich die Entscheidungsfindung dar (vgl. auch Dahlvik 2014: 254-303). Die Anhörenden waren in der Situation, auf die sich die Fluchtgeschichte bezieht, nicht selbst anwesend und kennen in der Regel auch das Herkunftsland nicht persönlich, sondern müssen sich auf vorliegende Informationen zur Lage in den Herkunftsländern (vgl. Gibb/Good 2013) und/oder die persönlichen Aussagen der Antragsteller*innen verlassen. Des Weiteren findet die sprachliche Verständigung im Wesentlichen über Dolmetscher*innen vermittelt statt (vgl. Scheffer 1997; Pöllabauer 2007; Gibb/Good 2014). Ein wesentlicher Teil des Arbeitsauftrags be- 
steht zudem darin, Prognosen über zukünftige Ereignisse zu treffen, ein per definitionem mit Unsicherheiten behaftetes Unterfangen (vgl. Staffans 2008: 621f.). Den Sachbearbeiter*innen liegen meist nur rudimentäre Informationen zum individuellen Fall vor. Das bedeutet, dass häufig in der Anhörung erst die Fakten produziert werden, die dann der weiteren behördlichen Bearbeitung zugeführt werden. Dafür gibt es in der Regel - für Sachbearbeiter*in und Antragsteller*in gleichermaßen - nur eine einmalige Gelegenheit, womit ein spezifischer Erfolgsdruck auf der Situation lastet. Hinzu kommen die für street-level bureaucracies charakteristischen Arbeitsbedingungen, die u.a. durch mangelhafte Ressourcen- und Personalausstattung und widersprüchliche Zielvorgaben gekennzeichnet sind (vgl. Lipsky 2010). Wie jede andere Form personen- bzw. fallbezogenen Arbeitens lässt sich auch das Anhören nicht auf reines Fachwissen reduzieren. Es bedarf vielmehr der praktischen Einübung. Individuelle und organisationale Routinen können dazu beitragen, Unsicherheiten und Komplexität zu reduzieren. Zugleich steht eine allzu routinierte Fallbearbeitung dem Erfordernis der individuellen Prüfung des Einzelfalls entgegen.

An dieser Stelle setzt das EASO-Modul `Gesprächsführungstechniken` an. Es gehört zu den sogenannten Kernmodulen des EASO Schulungsprogramms ${ }^{10}$ und vermittelt sowohl methodische Kenntnisse als auch Verhaltensregeln, die bei der Interaktion mit Asylantragsteller*innen zu beachten seien. Hierbei werden die Behördenmitarbeiter*innen zunächst explizit daran erinnert, dass sie gegenüber den Antragsteller*innen den Staat repräsentieren.

„Als Befragender sind Sie ein Vertreter Ihres Landes, das alle maßgeblichen Menschenrechtsinstrumente unterzeichnet hat. In dieser Eigenschaft müssen Sie professionell auftreten und Ihrer Aufgabe gewachsen sein. [...] Es kann nützlich sein, sich vor Augen zu führen, dass eine persönliche Anhörung kein zwangloses Gespräch, aber auch kein Verhör ist. Eine solche Anhörung muss im Rahmen eines fairen, respektvollen und effizienten Verfahrens erfolgen, das es dem Antragsteller je nach persönlicher Situation ermöglicht, seinen Fall umfassend darzulegen.« (ETC-Modul Gesprächsführungstechniken, Lektion 1.2)

Es geht hier v.a. um die Vermittlung eines Verständnisses von prozeduraler Gerechtigkeit; das Verfahren soll so angelegt sein, dass der Einzelfall sowohl umfassend dargelegt als auch angemessen gewürdigt werden kann. Anhörungen im Asylverfahren werden hier vom polizeilichen Verhör einerseits, von `natürlicher Kommunikation andererseits abgegrenzt. Sie sprofessionell durchzuführen, be-

$10 \mathrm{Zu}$ den beiden weiteren Kernmodulen des EASO Schulungsprogramms gehören $>$ Inclusion` (Schutzgewährung) und `Evidence Assessmentく (Beweiswürdigung). 
deutet, das Gespräch auf eine Weise zu führen, die - ähnlich dem sozialwissenschaftlichen Interview - geeignet ist, freie Erzählungen zu generieren. Neben Grundsätzen wie Respekt, Fairness und Objektivität wird im Modul die praktische Anwendung einer strukturierten, in Gesprächsphasen unterteilten Form der Befragung vermittelt. Unter Verweis auf Erkenntnisse der Kommunikationswissenschaften und der Psychologie (z.B. was interkulturelle Kommunikation, die Funktionsweise des menschlichen Gedächtnisses oder mögliche Folgen von Traumatisierungen für das Gesprächsverhalten betrifft) wird die Art und Weise, in der Anhörungen durchgeführt werden sollen, einer expliziten Methodisierung unterzogen. Dazu gehört auch, dass die Sachbearbeiter*innen aufgefordert sind, ihre Emotionen, Einstellungen und Verhaltensweisen zu reflektieren.

»Personen, die diese Verhaltensweisen an den Tag legen, wird häufig professionelles Arbeiten attestiert, d.h., der Befragende bleibt während der gesamten Anhörung unvoreingenommen und neugierig. Häufig können die eigene Einstellung und Möglichkeiten zu deren Beeinflussung nur mittels Selbstreflexion ermittelt werden.« (ETC-Modul Gesprächsführungstechniken, Lektion 2.2)

»Nach der Anhörung ist es sehr wichtig, dass Sie sich die Zeit für eine Selbstreflexion und Selbstanalyse nehmen. Wie ist die Anhörung für Sie und den Antragsteller gelaufen? Haben Sie sich respektvoll verhalten und die richtigen professionellen Verhaltensweisen angewendet?« (ETC-Modul Gesprächsführungstechniken, Lektion 8.1)

Mit den Empfehlungen zur systematischen Selbstbeobachtung, zu einer reflexiven Haltung gegenüber dem eigenen beruflichen Tun sowie zur Achtsamkeit mit Bezug auf das eigene Wohlergehen (vgl. auch EASO 2014b: 25) wirken die Schulungen den Standardisierungstendenzen bürokratischer Organisationen potentiell entgegen. Denn hier geht es v.a. darum, eigene wie behördliche Routinen der Fallbearbeitung zu hinterfragen und sich eine gewisse Offenheit für Handlungsalternativen zu bewahren bzw. zu erarbeiten. Gleichzeitig vermittelt das Modul eine Methode für einen Tätigkeitsbereich, der zuvor nicht explizit bis ins Detail geregelt war.

Was nun den Umgang mit den Schulungsinhalten in den beiden von uns untersuchten Behörden betrifft, so zeigten sich im Material grundlegende Gemeinsamkeiten, die wir kurz zusammenfassen möchten, bevor wir ausführlicher auf die Unterschiede zu sprechen kommen. Zum einen wird die Anhörung fall- und behördenübergreifend als berufliche (und persönliche) Herausforderung, als das Herzstück des Verfahrens, als das, was die meiste Kompetenz erfordere, beschrieben und in dieser Hinsicht von anderen Verfahrensschritten abgegrenzt. 
Zum anderen teilen die Befragten ein Wissen darüber, dass verhörähnliche Methoden als nicht mehr angemessen gelten und stellen dies im Interview entsprechend dar. Neben der allgemeinen Einschätzung, dass die neuen Methoden >fairer` oder >menschlicher seien, werden sie von ihnen v.a. daraufhin bewertet, inwieweit sie das Arbeiten (praktisch, moralisch, emotional) erleichtern und der Erledigung des Arbeitsauftrags dienen.

»wie gesagt auch Fragetechniken hatten wir ja da auch geübt und (also) dass man halt die ähm keine (.) keine geschlossenen Fragen fragt ne? (.) Kann man fragen aber sollte man nicht komplett fragen weil das bringt einen auch nix wenn er nur mit Ja oder Nein antwortet« $\left(\right.$ Entscheider*in Baier, Z. 1336-1375) ${ }^{11}$

Auch wenn die Entscheidung über den Antrag zu einem späteren Zeitpunkt getroffen wird und sich erste Eindrücke im Prozess der Bescheiderstellung durchaus ändern können, ${ }^{12}$ werden in der Anhörung wichtige Grundlagen für die Entscheidung geschaffen. Dies wird in dem diesem Beitrag vorangestellten Zitat aus dem BAMF deutlich zum Ausdruck gebracht, auf ganz ähnliche Weise formulieren aber auch schwedische Sachbearbeiter*innen:

»And the interview is of course of really central importance for the outcome of a decision, (3) and of course it is a question of (3) of getting the applicant to produce a good narrative and to ask the right questions. Indeed, the interview is of course really central, if you do a good interview then it might be fairly easy to write a decision from it.« (case officer Petersson, Z. 67-74)

$»[T]$ o write a decision from [an interview] « ist allerdings ein voraussetzungsreicher Prozess, bei dem es v.a. um die Transformation von Gesprochenem in Schriftliches geht (vgl. Scheffer 1998). Allein das, was während der Anhörung schriftlich protokolliert wurde, darf in der weiteren Fallbearbeitung relevant werden. In Asylverfahren gibt es zwei zentrale Momente einer solchen Trans-

11 Die Interviews wurden ohne die Verwendung von Satzzeichen transkribiert. Begriffe in Klammern stehen für eine Verständnisunsicherheit, Zahlen in Klammern für die Dauer der Pause in Sekunden, (.) steht für kurze Pausen. Bei sämtlichen Namen handelt es sich um Pseudonyme.

12 Z.B. weil sich im Anhörungsprotokoll doch nicht genügend Argumente für die ursprünglich angedachte Entscheidung finden, oder weil nach der Anhörung noch weitere Informationen aufgetaucht sind oder Recherchen durchgeführt wurden. 
formation: das Protokollieren (während und nach der Anhörung) ${ }^{13}$ (ebd.) und das Schreiben des Bescheids (während und nach der Entscheidungsfindung). Während für die Entscheidungsbegründung im Bescheid explizite formale Vorgaben

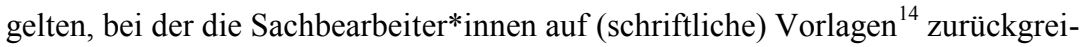
fen können, ist die Anhörungssituation aufgrund des Interaktionscharakters nie ganz vorhersehbar. »[G]etting the applicant to produce a good narrative« verweist auf die Verantwortung der Anhörenden für den Gesprächsverlauf und die dem Verfahren inhärenten Machtasymmetrien. Die vergleichende Analyse zeigte, dass sich sowohl der Prozess der Verschriftlichung als auch die Gestaltung der Interaktionssituation und die Art und Weise, wie Befragungstechniken in den beiden Behörden vermittelt, eingeübt, reflektiert und kontrolliert werden, wesentlich unterscheiden.

\subsection{Anhörungen in der schwedischen Migration Agency}

Die SMA ist dem Justizministerium und dem Migrationsminister zugeordnet und wird von der Regierung und dem Parlament beauftragt. Diese legen den Haushaltsplan der Behörde sowie - z.T. in Abstimmung mit dem Außenministerium die grundsätzlichen Ziele und Aufträge der schwedischen Asyl- und Migrationspolitik fest (vgl. Migrationsverket 2015, o.J.). Abgesehen von der formalen Rechenschaftspflicht gegenüber diesen beiden Organen ist im schwedischen Grundgesetz (2. Kap §2) die Unabhängigkeit der Verwaltungsbehörden verankert (vgl. Bull/Halje/Bergström/Reichel/Nergelius 2012; Yates 2000: 156) und auch die SMA bei der Erstellung ihrer Leitlinien weitgehend unabhängig. Im Vergleich zu Deutschland weist das Rekrutierungssystem des öffentlichen Dienstes in Schweden deutlich weniger Formalisierung und keine spezifischen staatlich festgelegten Einstellungsvoraussetzungen auf. Seit den 1960er Jahren wurde das Beamtentum schrittweise abgebaut und ist heute größtenteils abgeschafft (vgl. Hils/Streb 2010: 29). In der Migration Agency arbeiten hauptsächlich Personen mit einem abgeschlossenen Hochschulstudium in den Bereichen Jura und Politik- oder Sozialwissenschaften. Bezüglich der Altersstruktur ist die Behörde vergleichsweise jung (vgl. Migrationsverket 2014: 37).

13 In beiden Behörden sind die Sachbearbeiter*innen auch diejenigen, die die Anhörung protokollieren. Allerdings unterscheiden sie sich in den Techniken: In der SMA wird getippt, im BAMF meist diktiert.

14 In beiden Behörden spielen sogenannte >Gerüstbescheide` mit vorgefertigten Textbausteinen für unterschiedliche Fallkonstellationen und Entscheidungsarten eine wichtige Rolle in der täglichen Arbeit. 
Die Migration Agency ist für sämtliche Aspekte des Verfahrens (inkl. Unterbringung, Gesundheitsversorgung, Integrationsmaßnahmen, Arbeitserlaubnisse u.v.m.) zuständig. Zudem ist sie für den Vollzug der eigenen Entscheidungen, d.h. auch für die Durchführung von Abschiebungen und Rückführungsmaßnahmen, verantwortlich. Zum Zeitpunkt unserer Datenerhebung galt in der SMA, dass nach dem Prinzip der >lernenden Organisation « Verfahrenspraktiken im kollegialen Austausch stetig weiterentwickelt werden sollten. Zu diesem Zweck wurde u.a. ein Rotationsverfahren zum Wechsel zwischen verschiedenen Tätigkeiten und Abteilungen eingerichtet. In den Asylprüfungsabteilungen selbst ist das Team die für die Fallbearbeitung verantwortliche Basiseinheit. Hier können Fälle besprochen und konkrete Handlungsempfehlungen - auch zu anstehenden Anhörungen - gegeben werden.

Verantwortlichkeiten bei der Prüfung eines Asylantrags sind in der SMA funktional differenziert. Sachbearbeiter*innen, im Folgenden >case officers`, führen die Asylanhörungen durch, erstellen das Protokoll, stellen ggf. weitere Recherchen an und verfassen einen Entwurf zum Bescheid. Für die letztendliche Entscheidung tragen statushöhere Entscheider*innen, im Folgenden >decision makers $<$, die Verantwortung. ${ }^{15}$ Zwischen beiden besteht ein institutionalisierter Austausch. Die/der decision maker ist in der Anhörungssituation zwar nicht physisch präsent, verständigt sich jedoch vor, während und nach der Anhörung in eigens dafür eingerichteten Zeitfenstern mit der/dem case officer darüber, worauf im weiteren Verlauf geachtet werden sollte und welche Nachfragen gegebenenfalls noch gestellt werden sollten. Bei den meisten Anhörungen sind zudem von der Migration Agency gestellte - Rechtsberater*innen anwesend. Diese sollen die Interessen der Asylsuchenden vertreten und haben bspw. während der Anhörung die Möglichkeit, zusätzliche Fragen zu stellen und nach der Anhörung den Inhalt des Protokolls zu prüfen (vgl. European Migration Network 2012: 12). Vor der Bescheiderstellung werden die Eingaben (Englisch: submissions) der Rechtsvertreter*innen abgewartet und in der Entscheidungsfindung berücksichtigt.

Die Anhörungen bei der Migration Agency finden damit in einem Rahmen vergleichsweise hoher Beobachtbarkeit statt. Die Beteiligung der unterschiedlichen Akteure kann das Frageverhalten der case officers, den konkreten Verlauf einer Anhörung und die Entstehung des Protokolls wesentlich beeinflussen. Die decision makers sind darin sowohl Sozialisationsinstanz, über die berufliche Er-

15 In der Regel beginnen die Angestellten in den schwedischen Asylprüfungsabteilungen als case officers und steigen nach ein paar Jahren zum decision maker auf (Interviews mit Behördenmitarbeitenden). 
fahrung weiter vermittelt wird, als auch Instanz der Qualitätskontrolle. Der Austausch geschieht v.a. über direkte persönliche, d.h. mündliche, Kommunikation.

»Then you always have a dialogue with the decision maker. That is also important, that (6) that we take a break in the middle of the interview, when I go to the decision maker and kind of tell them that: this is what he has said, this is how I see it, the decision maker might say: can you ask a bit about this and a bit about that, and then you decide what further questions to ask, after the break, so you don't miss anything out, because if you miss anything out you might perhaps have to do a further interview. That takes time and money.« (case officer Petersson, Z. 514-524)

In der Wahrnehmung der hier zitierten Person kann dadurch der Gefahr, während der Anhörung etwas zu >verpassen`, begegnet werden. Die Verantwortung für die Vollständigkeit des in der Anhörung erfragten Sachverhalts wird als eine geteilte wahrgenommen. Mit der Unterbrechung des Interviews zum Zwecke dieser Absprachen wird die Anhörung bereits zum Gegenstand von Reflexion und Aushandlung. Dabei steht die Orientierung an Effizienzkriterien im Vordergrund. »[T]ime and money« für eine zweite Anhörung können gespart werden, wenn die Anhörung im ersten Anlauf alles für die Entscheidung Wichtige abdeckt. ${ }^{16}$ Zudem geht die Art der Interviewführung als ein Element in die leistungsbezogene Bewertung und Vergütung der Arbeit der case officers ein (case officer Petersson, Z.637-646). Da die case officers auch für die Erstellung eines Entwurfs zum Bescheid zuständig sind, wird die Gesprächsführung bereits mit Blick auf die später zu treffende Entscheidung ausgehandelt und reflektiert (case officer Sjöberg, Z. 264-282). Bei Unstimmigkeiten zwischen decision maker und case officer oder Unsicherheiten bezüglich der rechtlichen Sachlage wird häufig ein weiterer decision maker oder ein sogenannter Asylexperte (siehe auch Furre/Gustafsson/Quarles/Viblemo 2010: 91) einbezogen, bis Einigkeit über die zu treffende Entscheidung erreicht wird. Es geht bei der Entscheidungsfindung also v.a. um die Herstellung von Konsens. ${ }^{17}$ Aus Sicht der Befragten ist auch dies u.a. durch Effizienzerwägungen motiviert. Bei der Bescheiderstellung drehen sich

16 Interessant ist aber an dieser Stelle, dass überhaupt die Möglichkeit einer zweiten Anhörung erwähnt wird. In den Interviews mit Mitarbeitenden des BAMF ist dies nicht der Fall.

17 Die Suche nach Konsens wird von manchen als Kennzeichen der politischen Kultur Schwedens insgesamt gesehen. Diese orientiere sich an den Schlüsselbegriffen der Integration und Verhandlung (vgl. Jahn 2009: 134) und sei von einer pragmatischen »culture of consensus« (Pettersson 1994 zitiert in Yates 2000: 169) geprägt. 
diese v.a. darum, Zeit- und Personalressourcen so einzusetzen, dass Bescheide nicht mehrmals überarbeitet werden müssen und die Fälle nicht 〉steckenbleiben`:

»But it is (?) it is of course necessary if you want to have a well-functioning unit and you want to see the cases moving briskly, if they aren't to get stuck entirely, then you have to have that discussion, because after that I do a draft but there is no point in me working on a draft that a decision maker perhaps doesn't agree with. Instead we usually check it out, I mean we check it out with decision makers before the interview, during the interview, after the interview. And after the submission has come in and before we move to decision, and then we usually decide then and there what we are most likely going to write. And if we aren't certain at that point, then it's necessary to bring in someone else, sometimes all you need is to bring in another decision maker. So that there are three of you to talk it through to a conclusion or that you refer it to a team leader, or that we refer it to some expert.« (case officer Forsberg, Z. 608-626)

Gerade mit Blick auf den Umgang mit Unsicherheit spielt der Austausch unter den Mitarbeiter*innen eine wichtige Rolle. Indem in der SMA nicht nur im informellen Kollegenplausch, sondern systematisch und v.a. mit Blick auf die zu erbringende Leistung (die Erstellung eines Bescheids, dem mindestens case officer und decision maker zustimmen können) zusammengearbeitet wird, findet die Anhörung unter besonderen Voraussetzungen statt. Die Eindrücke des Gesprächs müssen nicht nur - in schriftlicher Form - den Anforderungen eines legitimen Verfahrens Genüge tun, sondern zunächst mündlich und auf eine auf die nahe Zukunft gerichtete Weise an die decision makers kommuniziert werden. Erst nach diesem Austausch und den Eingaben der Rechtsvertreter*innen wird mit der schriftlichen Begründung des Bescheids begonnen. Bezeichnenderweise zeigen die Interviews, dass die schwedischen Befragten dieser Art der Fallbearbeitung eine stark legitimitätsproduzierende Kraft zuschreiben. Unsicherheiten, Zweifel und ein Mangel an Informationen werden durch »discussion« und »talk« bearbeitet und zu kompensieren versucht.

"you need the discussion in any case, I think (.) you, somehow to be able to figure something out (5) so uhm (3) well, and there isn't always that much by way of country information and well but some assessments can be really difficult, particularly when it becomes a question of reliability and credibility because that part might be quite subjective but you have to try to get away from letting it be too subjective you have to do that. « (case officer Petersson, Z. 306-314) 
Die dem Asylverfahren inhärenten Unsicherheiten und das subjektive Element der Fallbearbeitung werden von den Befragten zwar anerkannt, Zweifel über die Richtigkeit einmal getroffener Entscheidungen werden in den Interviews allerdings kaum thematisch. Wie auch im oben zitierten Interview deutlich wird, nehmen die schwedischen Befragten die kontinuierliche Deliberation zwischen verschiedenen Akteuren als geeignetes Instrument wahr, um die Gefahr >allzu< subjektiver Entscheidungen zumindest einzuhegen.

\subsection{Anhörungen beim Bundesamt für Migration und Flüchtlinge}

Das deutsche Bundesamt ist als Bundesoberbehörde im Geschäftsbereich des Bundesinnenministeriums angesiedelt und an dessen Weisungen gebunden. Sowohl die Formulierung der politischen Leitlinien als auch die konkreten Vorgaben zum Verwaltungshandeln sind in hohem Maße von der Politik des jeweiligen Bundesinnenministeriums geprägt (Schneider 2012). Im Gegensatz zur SMA obliegt dem BAMF zwar die Durchführung des Asylverfahrens im engeren Sinne. ${ }^{18}$ Für sämtliche sonstigen Belange der Asylsuchenden (Fragen der Unterbringung, der sozialen Sicherung, der Erteilung von Arbeitserlaubnissen u.ä.) sowie für die Durchsetzung von Abschiebungsandrohungen nach ablehnendem Bescheid durch das BAMF sind jedoch die Bundesländer und Kommunen zuständig.

Die Zulassungsvoraussetzungen für die Tätigkeit im BAMF sind stark formalisiert und unterliegen den gesetzlichen Einschränkungen für die Anstellung im öffentlichen Dienst nach dem Laufbahnmodell. Für die Prüfung der Asylanträge sind Beamtinnen und Beamte oder Angestellte im gehobenen Dienst zuständig. Zum Zeitpunkt der Datenerhebung waren dies größtenteils Personen mit einer reinen Verwaltungslaufbahn oder Jurist*innen (persönliche Kommunikation mit dem Referat Personalqualifizierung, Dezember 2014). In diesem Bereich zeigen sich seit 2015 jedoch Öffnungstendenzen. Auf die neueren Stellenausschreibungen des Bundesamtes kann sich allgemein bewerben, wer über einen an einer Hochschule erworbenen Abschluss (mindestens Bachelor) verfügt. Zudem werden vermehrt befristete Verträge abgeschlossen und es wird Personal aus anderen Behörden (bspw. der Bundesagentur für Arbeit, dem Zoll, aber auch der Post u.a.) unterstützend eingesetzt. Aufgrund der seit Beginn der 1990er Jahre stark begrenzten Neueinstellungen im Entscheiderbereich (vgl. Kreienbrink

18 Daneben wurden dem Amt seit 2003 eine Reihe anderer Aufgaben in den Bereichen Migration, Integration, Forschung übertragen. 
2013) und anders als in der SMA geht der Personalaufbau im BAMF mit einem ausgeprägten Generationswechsel einher.

Im Unterschied zur schwedischen Asylbehörde gibt es im BAMF keine explizite personale Differenzierung zwischen den Tätigkeiten des Anhörens und des Entscheidens, d.h. die anhörenden Sachbearbeiter*innen sind oft auch diejenigen, die die Entscheidung treffen. Dies ist ein Grundsatz, an dem lange Zeit nicht nur seitens der Behörde, sondern auch von Gerichten, Vertreter*innen der Anwaltschaft u.a. festgehalten wurde, um zu vermeiden, dass rein nach Aktenanlage entschieden wird, wo es doch um individuelle Prüfung des Einzelfalls gehen soll (vgl. Deutscher Bundestag 2014). ${ }^{19}$ Bezeichnenderweise werden die Sachbearbeiter*innen im behördlichen Jargon denn auch schlicht als `Entscheider bezeichnet. Rechtsvertreter*innen nehmen nicht regelmäßig an Anhörungen teil; routinemäßig anwesende Dritte sind allein die Dolmetscher*innen (Feldnotizen BAMF Außenstellen). Im Behördenvergleich stellt die Anhörungssituation in Deutschland somit einen Bereich der tendenziellen Nicht-Beobachtbarkeit dar.

Institutionalisierte Formen organisationalen Lernens sind beim BAMF hauptsächlich auf die Einarbeitungsphase und punktuelle Fort- und Weiterbildungsmaßnahmen beschränkt. Eine direkte, auf die Anhörung bezogene $\mathrm{Zu}$ sammenarbeit mit Kolleg*innen oder Vorgesetzten ist die Ausnahme und beschränkt sich meist auf das Mentoring in der Einarbeitungszeit, während der man erfahreneren Kolleg*innen eine Zeitlang süber die Schulter schaut` und umgekehrt bei den ersten eigenen Anhörungen von ihnen begleitet wird. Anders als bei der SMA ist diese Phase jedoch zeitlich begrenzt. Schulungen und die sogenannten Entscheidertagungen bieten zwar weitere Gelegenheiten zum kollegialen Austausch, u.a. auch über Anhörungstechniken. Was den Arbeitsalltag betrifft, so findet ein solcher nach den Aussagen der Befragten im Regelfall nur eingeschränkt und außerhalb der Anhörungen statt. Zudem bezieht er sich eher auf die Entscheidungsfindung und -begründung als auf die Anhörungssituation selbst. In den Interviews kam dies beispielsweise wie folgt zum Ausdruck:

»Also w- ähm man spricht auch schon darüber dass man (.) $\mathrm{n}$ andern Anhörungsstil hat wobei das macht glaub ich (2) kann man ja auch nich ändern also das ist ja dann halt so dass jeder anders anhört und äh das überlässt hier glaub ich auch jeder ihm selbst wie er

19 Wobei davon auszugehen ist, dass dies mit der Einführung von >Ankunfts-८ und 〉Entscheidungszentren< und sonstigen Beschleunigungs- und Priorisierungsmaßnahmen inzwischen häufiger vorkommt. In der Antwort der Bundesregierung auf eine Kleine Anfrage der Linken ist für das zweite Quartal 2015 von ca. 25\% die Rede (Deutscher Bundestag 2015: 66). 
anhören möchte was man hier aber gut besprechen kann ist dann äh die Entscheidung an sich also man kann zu jedem gehen und sagen ähm ich hab hier son Fall der is so und so und der hat das und das gesagt was mach ichn da? (Entscheider*in Kriegel, Z. 491-500)

Unterschiedliche Anhörungsstile werden hier als etwas Gegebenes, nicht Veränderbares, aber auch nicht weiter Problematisches dargestellt. Was als Diskussionsgrundlage genutzt wird, sind die erinnerten Aussagen der Antragsteller*innen: »der hat das und das gesagt«. Ähnlich wie in Schweden wird der kollegiale Austausch über den Fall als ein mündlicher beschrieben. Hierbei geht es jedoch nicht darum, welche weiteren Fragen möglicherweise gestellt werden könnten oder sollten, denn der Austausch findet erst nach dem abgeschlossenen Anhörungstermin statt und dient allein dem Umgang mit Zweifel bezüglich der zu treffenden Entscheidung.

Eine weitere Variante des Umgangs mit Unsicherheiten besteht in der persönlichen Auseinandersetzung mit dem schriftlichen Protokoll, mit der Akte. Wie die folgenden Auszüge verdeutlichen, ist dies für die Befragten oftmals der erste Schritt, um Distanz zum Fall gewinnen. Erst danach werden ggf. Kolleg*innen um Rat gebeten.

»es is auch immer gut dann nachher noch ein Stückchen Abstand zu haben und dann noch mal in Ruhe das Protokoll auch wirklich zu lesen und ähm (.) wenn man manchmal auch noch nen Zweifel hat (dann) liest man's noch mal oder spricht mit nem Kollegen drüber der hat manchmal auch noch $\mathrm{n}$ anderen Blick und dann halt die Entscheidung zu treffen« (Entscheider*in und Trainer*in Krüger, Z. 230-236)

»mal noch jemand anderes das Protokoll lesen lassen oder das was der Anwalt schreibt ähm lesen lassen um einmal andere Gedanken dazu zu hören das hilft auf jeden Fall« (Entscheider*in und Trainer*in Gabriel, Z. 1042-1045)

Austausch findet also durchaus statt, bezieht sich auf die Gesprächsführung jedoch nur indirekt und ex post. Der Prozess der Entscheidungsfindung schreitet allein auf Grundlage des schriftlichen Dokuments weiter voran. Insbesondere für Neueingestellte bietet der Austausch mit erfahreneren Kolleg*innen auf Basis des Protokolls zwar auch Gelegenheiten zur Reflexion des eigenen Frageverhaltens und zu möglichen, zukünftigen Veränderungen (Entscheider*in Baier, Z. 1421-1463). In der Anhörungssituation selbst tragen die Sachbearbeiter*innen jedoch die alleinige Verantwortung dafür, die richtigen Fragen auf die richtige Art und Weise und im richtigen Moment zu stellen. 
Außerhalb der Einarbeitungsphase erhalten die Sachbearbeiter*innen beim BAMF in der Regel nur dann Einblick in die Arbeitsweisen der Kolleg*innen, wenn sogenannte fremdangehörte Fälle entschieden werden müssen. Dieser Einblick ist wiederum auf die Niederschrift zur Anhörung begrenzt. Hier beurteilen einige der Befragten das Vorgehen mancher Kolleg*innen durchaus kritisch. Mit der Begründung, dass es sich unter Gleichen nicht schicke, Kritik an den Arbeitsweisen der jeweils anderen zu üben, dass nur über Anweisungen ein wirklicher Einfluss ausgeübt werden könne und unter Bedingungen von Personalmangel ohnehin nicht auf die Kolleg*innen verzichtet werden könne, werden entsprechende Bedenken jedoch weder an die betreffenden Kolleg*innen noch an Vorgesetzte kommuniziert (Entscheider*in und Trainer*in Krüger, Z. 13791428; Entscheider*in Mayer, Z. 292-383).

Neben den Arbeits- und Zuständigkeitsverteilungen innerhalb der Behörde hängt dies auch mit organisationskulturellen Narrationen über das, was den/die Entscheider*in ausmacht, zusammen: Trotz Abschaffung der Weisungsungebundenheit mit dem Zuwanderungsgesetz in 2005 (§ 5, 6 AsylVfG) wird sowohl in den Interviews als auch bei den von uns beobachteten Schulungen weiterhin das richterähnliche Bild des `Einzelentscheiders〈 (so die frühere Bezeichnung) heraufbeschworen. Dies entspricht der deutschen Tradition des Offizialprinzips, nach dem der Entscheider derjenige ist, der die alleinige Kontrolle über Art und Ausmaß der im Verfahren zu sammelnden und beurteilenden Beweise hat (vgl. Staffans 2008: 632-640). In der beruflichen Praxis gilt das Prinzip des NichtEinmischens in das, was die Kolleg*innen tun (vgl. Scheffer 2003: 451f., der den Entscheider-Stand in dieser Hinsicht mit dem der Ärzte, Richter oder Hochschullehrer vergleicht). Damit befinden sich die deutschen Sachbearbeiter*innen in einer Situation, in der sie einerseits großer Kontrolle - über Dienstanweisungen, Leitsätze und Priorisierungsmaßnahmen - unterworfen sind, gleichzeitig jedoch von ihnen gefordert wird, individuell Verantwortung für die Einhaltung der für die Durchführung von Anhörungen vorgegebenen Standards zu übernehmen. $^{20}$

Im Vergleich zur Anhörung wird der Prozessschritt der Entscheidung beim BAMF stärkerer expliziter Kontrolle unterzogen. Qualitätskontrollen finden bezogen auf das letztendliche >Produkt` der Fallbearbeitung statt, nämlich in Form einer Kurzübersicht zum Bescheid, die von sogenannten Qualitätsförderern in den Außenstellen überprüft und abgezeichnet wird. Diese Kontrolle ist meist auf

20 Während der von uns beobachteten Schulungen wurde denn auch wiederholt kritisiert, dass die Qualitätsansprüche aus der Zentrale unter den vor Ort herrschenden Arbeitsbedingungen kaum zu erfüllen seien. 
formale Aspekte, die Plausibilität der getroffenen Entscheidung und ihre Übereinstimmung mit den behördlichen Vorgaben fokussiert (Interview Altmann, Z. 471-481). Die Verantwortung für die Vollständigkeit des erfragten Sachverhalts und für die inhaltliche Prüfung der Glaubhaftigkeit des Vortrags der Asylsuchenden wird weitgehend den Entscheider*innen überlassen. ${ }^{21}$ Lediglich in Fällen, die aufgrund der getroffenen Entscheidung oder eines besonders gelagerten Sachverhalts >auffällig`sind, wird Einblick in den vollständigen Bescheid, in noch selteneren Fällen in das Anhörungsprotokoll genommen (Feldnotizen BAMF Außenstellen, Gespräche mit Qualitätsförderern).

Es zeigt sich hier, dass die organisationale Antwort auf das Problem des Entscheidens unter Bedingungen von Ungewissheit im BAMF v.a. darin besteht, einerseits auf die Kompetenzen der einzelnen Entscheider*in und andererseits auf die besondere Bedeutung der persönlichen Interaktion in der Anhörungssituation als Erkenntnisquelle zu vertrauen. Dies wurde auch bei den von uns beobachteten Schulungen für Asylsachbearbeiter*innen deutlich. Wenn die Teilnehmenden Unsicherheiten bezüglich der Beurteilung der Glaubhaftigkeit äußerten, wurde wiederholt darauf verwiesen, dass genau dies den Kern ihrer Tätigkeit ausmache: ") das ist Ihre Aufgabeく, >deswegen ist das so hochqualifiziert<, >da müssen Sie sich durchbeißen`, >da gibt's kein Patentrezept«« (Feldnotizen BAMF, Schulung 1, S. 21). Administrative Sachlichkeit und Objektivität werden aus dieser Sicht gerade durch das Prinzip des Nicht-Einmischens (weder durch Vorgesetzte, noch durch Kolleg*innen) sichergestellt. Darin drückt sich ein bemerkenswert stabiler Glaube an die leidenschaftslose Bürokratin aus, die allein aufgrund ihrer Sozialisation in die Rolle und aufgrund ihres spezifischen Fachund Dienstwissens neutrale Entscheidungen treffen kann.

21 Ausnahmen sind stichprobenartige Kontrollen durch das Referat Qualitätssicherung in der Zentrale. 


\section{4 `Ordentliche` Anhörungen im Vergleich}

As the quintessential form of legitimized discourse, the law can exercise its specific power only to the extent that it attains recognition, that is, to the extent that the element of arbitrariness at the heart of its functioning (which may vary from case to case) remains unrecognized. The tacit grant of faith in the juridical order must be ceaselessly reproduced.

(BOURDIEU 1987: 844, HERV. I.O.)

Asylsachbearbeiter*innen beider Behörden sind mit dem gleichen Kernproblem konfrontiert, der Unentscheidbarkeit von Entscheidungen (vgl. Fischer-Lescano/ Christensen 2005). 〉Guteく Anhörungen sind in der Wahrnehmung der Befragten von besonderer Bedeutung für >gute « Entscheidungen. Erstere werden von ihnen v.a. darüber definiert, zu welchem Grad es bereits während der Anhörung gelingt, genügend Informationen für die Entscheidungsbegründung zu sammeln. Im Kontext einer zunehmenden Methodisierung und Verwissenschaftlichung der behördlichen Praktiken, u.a. im Rahmen von Schulungen, wird das Frageverhalten der Sachbearbeiter*innen zum Objekt von Reflexion. Die bei den Schulungen vermittelten Ziele und die dafür zu verwendenden Methoden beziehen sich v.a. auf die Anforderungen prozeduraler Gerechtigkeit (i.S.v. Fairness und Respekt gegenüber den Antragsteller*innen) einerseits, die wissenschaftlich untermauerte und methodisierte Hervorbringung von für die administrative Entscheidungsfindung und -begründung verwendbaren Erzählungen andererseits. Beide Aspekte verweisen auf die Beziehungen zwischen Amt und >Umwelt .

Aus der Perspektive der Sachbearbeiter*innen präsentieren sich diese als teils konfligierende Anforderungen aus unterschiedlichen sozialen Feldern. Asylentscheidungen müssen sowohl rechtmäßig (oder zumindest möglichst >gerichtsfest $<$ ) sein als auch in der Summe den jeweils aktuell politisch gesetzten Vorgaben (bezüglich 〉Effizienz`, aber auch bestimmter >outcomes $`$ ) mindestens so weit entsprechen, dass sich die Behörde als ausführendes Organ des Staates nicht selbst delegitimiert. Für die gerichtliche Überprüfung der behördlichen Entscheidungen stellen die schriftlichen Produkte der Sachbearbeitung jedoch meist die alleinige Grundlage dar. Bei der Umsetzung europäischen Rechts ist die Transformation der Anhörung in das Protokoll und schließlich in den Asylbescheid somit von zentraler Bedeutung. Während sich Protokolle und Bescheide über explizite Vorgaben und Vorlagen steuern lassen, entzieht sich die Anhö- 
rung immer wieder den Versuchen zu ihrer Standardisierung. Dies ist der Moment, auf den sich der Glaube an und das Vertrauen in die behördlichen Verfahrensweisen beziehen (wenn wir die Bourdieu'sche Formulierung einmal so nonchalant auf die Verwaltung übertragen dürfen). Wie wir oben gezeigt haben, unterscheiden sich die beiden Behörden wesentlich mit Blick auf die Art und Weise, wie der »tacit grant of faith« in die eigenen behördlichen Entscheidungen über Schutzgewährung kollektiv hergestellt wird.

In der SMA dominieren deliberative Formen der Fallbearbeitung, die pragmatisch und auf die Herstellung von Konsens über die zu treffende Entscheidung ausgerichtet sind. Schriftliche Dienstanweisungen und Leitsätze spielen dort zwar eine wichtige Rolle, vieles wird aber mündlich verhandelt und zwar sowohl während der Anhörung als auch vor der schriftlichen Bescheiderstellung. Unterschiedliche Deutungen des Sachverhalts und Konflikte über die Entscheidungsfindung werden als Problem wahrgenommen, das gelöst (oder zumindest bearbeitet) werden muss, indem immer weitere Akteure in die Fallbearbeitung mit einbezogen werden. Unsicherheiten und Ambivalenzen werden im Verfahren somit auf verschiedenen Ebenen kleingearbeitet. Dies betrifft 1) die zeitliche Dimension (Unterbrechung der Anhörung, Absprachen vorher und nachher) und 2) die beteiligten Akteure (Antragsteller*innen, Dolmetscher*innen, Vorgesetzte, Kolleg*innen und Rechtsvertreter*innen) mit ihren jeweiligen Einflussnahmen auf das Frageverhalten und das schließlich erstellte Protokoll. In der Wahrnehmung der Befragten führt diese Art der Sachbearbeitung zu weitgehend homogenen Deutungs- und Denkweisen innerhalb der Behörde. In den Interviews wurden keine expliziten Abgrenzungen zwischen unterschiedlichen Anhörungsmethoden vorgenommen, im Gegenteil: »I think because we talk so much to each other and because we informally teach each other er we actually (1.6) er get to think in the same way« (trainer and case officer Mårtensson, Z. 1145-1147, siehe auch trainer Eklund, Z. 1090-1092). Im Interviewmaterial zeigte sich ein hohes Maß an (Selbst-)Vertrauen in die Güte des schwedischen Verfahrens mit seinen $>$ checks and balances $\measuredangle$. Etablierte Routinen wurden selten hinterfragt und die sneuen Vorgaben für die Durchführung von Anhörungen als eher schrittweise und kleinteilige Anpassung an eine bereits bestehende Praxis charakterisiert was auch damit zusammenhängt, dass die schwedische Behörde an der Konzeptionierung einzelner EASO-Module maßgeblich beteiligt war und vornehmlich jene umgesetzt wurden, die der eigenen Praxis weitgehend entsprachen (Gespräche mit Behördenvertreter*innen, November 2013). Bei der Entwicklung von Deutungen darüber, was >gute` Arbeit ausmacht, sind der tägliche direkte Kontakt zu den decision makers und das Zusammenarbeiten am konkreten Fall prägender als vereinzelte Schulungen. In gewisser Weise ist das schwedische Mo- 
dell damit hermetischer, denn das Team und insbesondere die decision-makers fungieren als \Puffer zwischen case officers und externen Anforderungen. Das lässt einen abrupten Wandel, ein schnelles sich-Anpassen an veränderte Umweltbedingungen insgesamt unwahrscheinlicher erscheinen.

Im BAMF dominiert das Prinzip der Aktenmäßigkeit. Kontrollen werden über hierarchische Befehlsketten zum einen vor der Anhörung (in Gestalt von Dienstanweisungen, Leitsätzen, Fragebögen usw.), zum anderen nach der Anhörung (vornehmlich in Gestalt des Abzeichnens der Kurzübersicht zum Bescheid) wirksam. Über die rasche und weitgehend individuelle Transformation von Gesprochenem in Schriftliches werden die Unwägbarkeiten der Interaktionssituation zum Verschwinden gebracht. Es ist hauptsächlich an einzelnen Sachbearbeiter*innen, in der konkreten Situation >professionelk zu handeln und die richtigen Fragen zu stellen. Damit ist das Verfahren dezisionistischer angelegt. Beim Umgang mit Zweifel wird zunächst v.a. auf Aushandlung der Entscheider*innen mit sich selbst (bzw. mit dem von ihnen produzierten Protokoll) gesetzt. Anhörungspraktiken werden von den Befragten denn auch als eher heterogen beschrieben. Qualitätsstandards scheinen insofern umkämpft, als es nicht die eine, kollektiv geteilte, Sicht auf das, was eine >ordentliche « Anhörung auszumachen habe, gibt. In den Interviews und auch bei den von uns beobachteten Schulungen sind Formen der Abgrenzung zwischen dem >alten` und dem >neuen`System, zwischen den >Harten $<$ und den >Soften< sehr ausgeprägt.

»ich hab $\mathrm{n}$ Kollegen ähm kennengelernt im Laufe meiner Zeit beim Bundesamt der so das Motto hat äh er möchte gerne durch gezielte Provokation äh eine Atmosphäre schaffen die dazu geeignet is das Lügengerüst zum Einsturz zu bringen also das is im Prinzip diametral entgegengesetzt zu dem was ich mir so als ähm Grundkonzept für ne Anhörungssituation vorstelle« (Entscheider*in und Trainer*in Gabriel, Z. 2553-2561)

Auf eine sehr viel explizitere Weise als in der SMA werden die Schulungen von den Befragten im BAMF mit einem intentional gesteuerten Wandel sowohl des Selbstbildes der Behörde als Ganzer wie der in der Fallbearbeitung angewandten Methoden in Verbindung gebracht. Dieser Wandel wird, anders als in der SMA, als deutlicher Bruch mit früheren Verfahrensweisen charakterisiert.

»dass man halt dann den neuen Mitarbeitern und Mitarbeiterinnen das nötige Handwerkszeug an die Hand gibt und im Idealfall ein paar Best Practices vermittelt äh und eben nicht ähm ähm mit Methoden arbeitet die vielleicht auch nicht mehr dem Leitbild des Amtes entsprechen « (Entscheider*in und Trainer*in Gabriel, Z. 637-642, vgl. auch Entscheider*in Mayer, Z. 228-237) 
Damit sind Anhörungspraktiken im BAMF zu einem größeren Ausmaß Gegenstand von Auseinandersetzungen und Deutungskämpfen. Mit der Objektivierung neuer Standards der Fallbearbeitung in den Schulungsprogrammen werden bestimmte Praktiken als erwünscht, andere als unerwünscht markiert. Diese Deutung wird von Führungskräften und Trainer*innen auch übernommen und an andere vermittelt. Da die Basis für die weitere Fallbearbeitung und auch für etwaige Qualitätskontrollen (durch Vorgesetzte oder die Gerichte) aber allein das schriftliche Protokoll darstellt, hängt es im hohen Maße vom beruflichen Selbstverständnis der individuellen Sachbearbeiter*innen ab, was genau unter einer sprofessionell durchgeführten Anhörung verstanden wird und inwieweit solche Deutungen handlungsleitend werden (können). Wenn Einzelne den neuen Anforderungen nicht entsprechen, so hat dies keine direkten Auswirkungen auf die Arbeit der Kolleg*innen - es handelt sich dann eben um die berühmten faulen Äpfel im Korb. Die Ansprüche an die Entscheidertätigkeit sind im BAMF also insofern höher, als es dort wesentlich auf die individuelle Entwicklung einer sprofessionellen` Haltung ankommt, für die die Sachbearbeiter*innen selbst verantwortlich sind. Für Neueinsteiger*innen können die Schulungen insofern unter Umständen relativ direkte Effekte haben. Bis zu welchem Grad die dort vermittelten Methoden in die täglichen Arbeitsroutinen inkorporiert werden, hängt dann v.a. von Selektionsprozessen bei der Rekrutierung und den Arbeitsbedingungen vor Ort ab.

Welche Implikationen haben diese Unterschiede nun für die Frage nach dem Verhältnis zwischen externen Vorgaben und Wandel auf dem street-level? Wie unter 3.1 dargestellt, vermittelt das EASO-Modul zu Gesprächsführungstechniken zum einen ein konkretes Methodenwissen, zum anderen Deutungen >professionellen Arbeitens`, die den für Anhörungen verantwortlichen Personen die Fähigkeit und Bereitschaft zur Selbstreflexion abverlangen. Allzu starre Routinen werden insofern als gefährlich angesehen, als sie einer ausreichenden Würdigung des Einzelfalls im Wege stehen können. `Gute` Anhörungen und Selbstreflexion erfordern aus Sicht der Befragten aber v.a. Zeit. Für beide Behörden galt bereits zum Zeitpunkt unserer Datenerhebung, dass Qualitätsanforderungen mit jenen der >Produktivität $\triangleleft$ in teils erheblichem Konflikt standen. Je nachdem, welche Aspekte der Sachbearbeitung bei der Bewertung von Arbeitsleistungen im Vordergrund stehen bzw. überhaupt >gemessen` werden, entwickeln Sachbearbeiter*innen Strategien, mit solchen Zielkonflikten umzugehen, die formalen Standards entgegenstehen können. Dies haben die Forschungen in der Tradition der street-level bureaucracy bereits umfassend herausgearbeitet und hat sich auch in unseren Arbeiten bestätigt (vgl. Schittenhelm 2015). Die Sachbearbeiter*innen in den beiden hier untersuchten Behörden können sich dabei auf unter- 
schiedliche institutionalisierte Quellen der Rechtfertigung stützen. So kann aufgrund der föderalen Differenzierung des deutschen Asylverfahrens die Verantwortung für manche Bereiche des Verfahrens, insbesondere was Fragen der Unterbringung, der sozialen Sicherung und der (Nicht-)Durchsetzung von Abschiebungsandrohungen betrifft, auf die Bundesländer und Kommunen ausgelagert werden. In Verbindung mit dem in der Regel nur zweimaligen Kontakt zu den Antragsteller*innen (bei der Antragstellung und bei der Anhörung) ermöglicht dies Strategien der Distanzierung von den Folgen der eigenen Entscheidungen. In den Bereichen, für die die BAMF-Mitarbeitenden im engeren Sinne zuständig sind, werden sie jedoch in hohem Maße auf ihr persönliches Gewissen einerseits, die politisch-rechtlichen Vorgaben andererseits, verpflichtet.

Die Migration Agency dagegen ist für sämtliche Aspekte des Verfahrens zuständig (vgl. Furre/Gustafsson/Quarles/Viblemo 2010: 90) und in direkter Weise verantwortlich für die Durchsetzung der eigenen Entscheidungen. Damit haben dort nicht einzelne Sachbearbeiter*innen, wohl aber die Behörde als Ganze einen häufigeren direkten Kontakt zu den Antragsteller*innen. Positive wie ablehnende Entscheidungen werden ihnen in und von der Behörde persönlich mitgeteilt. Innerhalb der Behörde liegt, wie wir oben gezeigt haben, die Verantwortung für das Treffen der Entscheidung jedoch nicht bei Einzelnen, sondern ist auf die Mitglieder des Teams und/oder auf die in der Anhörungssituation anwesenden Akteure aus verschiedenen Feldern verteilt. Damit werden potentielle Konflikte und Widersprüche bereits im Verfahren >verarbeitet‘. Das Endprodukt erscheint als ein >kooperativ und auf >gerechte Weise hergestelltes, die/der einzelne* $r$ Sachbearbeiter*in ist niemals allein verantwortlich.

Die im Schulungsmodul enthaltenen Anrufungen von sprofessioneller Arbeit entfalten damit in den beiden Behörden unterschiedliche Wirkungen. Zwar teilen die Befragten ein Wissen darüber, welche Befragungstechniken aus >europäischer Sicht als angemessen gelten und welche nicht. Da sich die »regimes of accountability on the street-level« (Hupe/Hill 2007: 292) der beiden Organisationen grundlegend unterscheiden, wird dieses Wissen aber jeweils selektiv und mit Blick auf die Lösung konkreter, praktischer Probleme eingesetzt und in situierten, kollektiven, teils konflikthaften Prozessen re-interpretiert.

\section{Ausblick}

Wie wir in diesem Beitrag gezeigt haben, sind die Wege, über die externe Vorgaben, neue Deutungen und neues Wissen Eingang in das Verwaltungshandeln finden können, in erheblichem Maße von organisationalen Bedingungen geprägt, 
die sich einer direkten Einflussnahme >von oben` entziehen. In besonderer Weise gilt dies für die direkten persönlichen Interaktionen zwischen Asylsuchenden und Behördenmitarbeiter*innen. Am Beispiel des ETC-Schulungsprogramms wurde deutlich, dass >europäische` Wissensbestände und Deutungen durchaus auf dem street-level ankommen. Die im Schulungsmodul enthaltenen Aufforderungen erfahren dort jedoch spezifische Transformationen. Wandel findet also statt, es geht dabei jedoch nicht um Konvergenz, sondern eher um inkrementelle Veränderungen bereits bestehender Praktiken. Form und Ausmaß der Veränderungen scheinen dabei wesentlich davon abzuhängen, wie das Verhältnis zwischen >frontline workers`, 〉peers $`$, direkten Vorgesetzten und Management strukturiert ist.

Zukünftige Arbeiten müssen zeigen, ob die oben dargestellten unterschiedlichen Umgangsweisen mit dem zentralen Handlungsproblem: dem Treffen von Entscheidungen unter Bedingungen von Ungewissheit, auch Einfluss auf die Anerkennungsquoten haben. In Schweden wurde 2015 bei einer Gesamtschutzquote von $72 \%$ in $40 \%$ der positiven Entscheidungen der Flüchtlingsstatus nach der Genfer Konvention, in $60 \%$ subsidiärer oder humanitärer Schutz vergeben (EUROSTAT 02.03.16, eigene Berechnungen). ${ }^{22}$ In früheren Jahren lag die Quote zu Letzteren - und zwar weit über dem europäischen Durchschnitt - noch höher (ebd.). Neben materiellen und rechtlichen Nachteilen stellt die eher restriktive Vergabe des >vollen ‘ Flüchtlingsstatus v.a. eine symbolische Exklusion derer dar, die nicht zu den >echten` Flüchtlingen zählen (vgl. Wettergren/ Wikström 2014; Hagelund 2003 zitiert in Schoultz 2014: 220). Zugleich konnte bis zuletzt mit der recht großzügigen Vergabe subsidiären und humanitären Schutzes das Selbstbild des schwedischen Staates als menschenrechtsorientiert und liberal aufrechterhalten werden. ${ }^{23}$ Die konsensorientierte Form der Fallbearbeitung befördert dabei die in Schweden beobachtbare Tendenz, Ambivalenzen

22 Für die grobe Darstellung der Verteilung der Entscheidungsarten stützen wir uns hier auf jährlich aggregierte EUROSTAT-Daten. Diese weichen allerdings von den nationalen Statistiken nicht unerheblich ab. Zudem sind Gesamtschutzquoten mit Vorsicht zu genießen. Für eine differenzierte Analyse sind die Entscheidungsquoten mit Bezug auf einzelne Herkunftsländer darzustellen; exemplarisch siehe dazu Fußnote 30.

23 Der »Paradigmenwechsel« (Parusel 2015b) der schwedischen Asylpolitik, der bspw. Einschränkungen im Familiennachzug sowie Maßnahmen zur Schließung und rigorose Identitätskontrollen an den Grenzen im Herbst 2015 und die konsequentere Umsetzung von tausendfachen Abschiebungsbeschlüssen beinhaltete (vgl. Svensson/Mattsson/Magnusson 2016), bringen dieses Selbstbild stark ins Wanken. 
dadurch aufzulösen, für die ssicherste Option` zu votieren (vgl. Wettergren/Wikström 2014: 570).

Im BAMF dagegen geht es - bei einer insgesamt niedrigeren, wenn auch im Jahr 2015 im historischen Vergleich sehr hohen Gesamtschutzquote von 57\% (EUROSTAT 02.03.16, eigene Berechnungen) - eher um salles oder nichts<. Hier wurde lange Jahre in der überwiegenden Mehrzahl der Fälle der Flüchtlingsstatus nach der Genfer Flüchtlingskonvention (GFK) bzw. nach Art. 16aGG festgestellt (vgl. Bundesamt für Migration und Flüchtlinge 2015a: 43-52, 2015b). In 2015 traf dies auf 97\% der positiven Entscheidungen zu, wohingegen bei lediglich 3\% Anspruch auf subsidiären Schutz bzw. Abschiebeverbote nach nationalem Recht festgestellt wurde (EUROSTAT 02.03.16, eigene Berechnungen). ${ }^{24}$

Auch wenn beide Behörden sich in der Begründung der Bescheide v.a. auf europäische Rechtsgrundlagen beziehen (von humanitärem Schutz nach nationalem Recht wird selten Gebrauch gemacht), zeigt sich hier, dass mit der Unterscheidung zwischen >echten` Flüchtlingen und >den anderen` in der Praxis sehr unterschiedlich umgegangen wird. ${ }^{25}$ Für solche Unterschiede gibt es eine Reihe möglicher Erklärungen. In der Forschungsliteratur wurde u.a. auf die Rolle von Parteien, nationalen Asylpolitiken, Antragszahlen und ökonomischen Faktoren in den Zielländern hingewiesen (vgl. Neumayer 2005; Vink/Meijerink 2003; Holzer/Schneider 2002). Die dort diskutierten Zusammenhänge konnten allerdings v.a. im Zeitverlauf nicht uneingeschränkt bestätigt werden. Um auch eher stabile Muster unterschiedlicher Anerkennungspraktiken erklären zu können, die sowohl von der jeweiligen Regierungspartei als auch von der Lage in den jeweiligen Herkunftsländern relativ unabhängig zu sein scheinen, plädieren wir dafür,

24 Hier zeigen sich jedoch ebenfalls - politisch induzierte - Veränderungen. Ein prominentes Beispiel hierfür ist die >Umstellungく der Entscheidungspraxis mit Bezug auf syrische Antragsteller*innen, die nun vermehrt subsidiären Schutz (inkl. der damit verbundenen Einschränkungen im Familiennachzug) und nicht mehr den Flüchtlingsstatus nach der GFK erhalten.

25 Die hier beschriebene allgemeine Tendenz zeigt sich auch, wenn bspw. nur die Anerkennungsquoten für Syrien betrachtet werden: 2015 wurde bei einer Schutzquote von $97 \%$ in beiden Ländern in Deutschland bei annähernd 100\%, in Schweden in lediglich $10 \%$ der positiven Entscheidungen der Flüchtlingsstatus nach der GFK vergeben (EUROSTAT 02.03.16, eigene Berechnungen). Bei Antragsteller*innen aus dem Irak unterscheidet sich schon die Gesamtschutzquote erheblich (98\% in Deutschland, 37\% in Schweden). Aber auch hier beinhalten die positiven Bescheide in Deutschland zu 98\% den GFK-Status, in Schweden zu 53\%. 
die organisationalen Bedingungen der Sachbearbeitung vor Ort mit in den Blick zu nehmen. Jenseits formaler Vorgaben entfaltet die lokale Verwaltungspraxis eine Eigenlogik, der mit einem Fokus auf rechtliche wie politische Steuerung und Standardisierung allein nicht beizukommen ist (vgl. Lahusen 2016). Und auch die eher >weichen`Maßnahmen der Verwaltungskooperation können sich, wie wir oben am Beispiel der Anhörungen diskutiert haben, sehr unterschiedlich auswirken, je nachdem, wer in den jeweiligen Behörden unter welchen Bedingungen für die Asylsachbearbeitung zuständig ist. Wir konnten hier nur erste Anhaltspunkte für einen Behördenvergleich auf der Ebene der Verwaltungspraktiken liefern. Zukünftige Analysen müssten den Sachbearbeitungsprozess als Ganzen - von der Antragstellung bis zur Bescheiderstellung - zum Gegenstand haben und systematisch überprüfen, in welchem Verhältnis die genannten Faktoren $\mathrm{zu}$ den verschiedenen, in der Verwaltungspraxis relevanten Wissensformen stehen. Für die Rechtstellung der Asylsuchenden und für die Überprüfbarkeit der behördlichen Entscheidungen ist diese Frage von zentraler Bedeutung.

\section{Literatur}

Bannink, Duco/Six, Frédérique/van Wijk, Eelco (2015): »Bureaucratic, market or professional control? A theory on the relation between street-level task characteristics and the feasibility of control mechanisms «, in: Peter $\mathrm{Hu}$ pe/Michael Hill/Aurèlien Buffat (Hg.), Understanding Street-level Bureaucracy, Bristol/Chicago: Policy Press, S. 205-226.

Barley, Stephen R./Kunda, Gideon (2001): »Bringing Work Back In«, in: Organization Science 12, S. 76-95.

Bendel, Petra (2013): Nach Lampedusa: das neue Gemeinsame Europäische Asylsystem auf dem Prüfstand, Studie im Auftrag der Abteilung Wirtschaftsund Sozialpolitik der Friedrich-Ebert-Stiftung (=WISO Diskurs), Bonn: Friedrich-Ebert-Stiftung.

Bohnsack, Ralf (2010): Rekonstruktive Sozialforschung, Stuttgart, [s.1.]: UTB GmbH; Barbara Budrich.

Bourdieu, Pierre (1987): »The Force of Law: Toward a Sociology of the Juridical Field«, in: The Hastings Law Journal 38, S. 805-853.

- (1994): »Rethinking the State. Genesis and Structure of the Bureaucratic Field «, in: Sociological Theory 12, S. 1-18.

Brekke, Jan-Paul/Brochmann, Grete (2015): »Stuck in Transit: Secondary Migration of Asylum Seekers in Europe, National Differences, and the Dublin Regulation«, in: Journal of Refugee Studies 28, S. 145-162. 
Brodkin, Evelyn Z. (2008): »Accountability in Street-Level Organizations«, in: International Journal of Public Administration 31, S. 317-336.

— (2011): »Putting Street-Level Organizations First. New Directions for Social Policy and Management Research«, in: Journal of Public Administration Research and Theory 21, S. i199-i201.

Bull, Thomas/Halje, Lovisa/Bergström, Marie/Reichel, Jane/Nergelius, Joakim (2012): Arvet från Oxenstierna - reflektioner kring den svenska förvaltningsmodellen och EU, SIEPS 2012:2op, Stockholm.

Clarke, Adele E. (2012): Situationsanalyse. Grounded theory nach dem Postmodern Turn (= Interdisziplinäre Diskursforschung), Wiesbaden: Springer VS.

Crawley, Heaven/Lester, Trine (2004): Comparative analysis of gender-related persecution in national asylum legislation and practice in Europe, Geneva.

Dahlvik, Julia (2014): Administering Asylum Applications, Unveröffentlichte Dissertation, Universität Wien.

Deutscher Bundestag (2014): Antwort der Bundesregierung auf die Kleine Anfrage der Abgeordneten Ulla Jelpke, Jan Korte, Sevim Dağdelen, weiterer Abgeordneter und der Fraktion DIE LINKE. Ergänzende Informationen zur Asylstatistik für das Jahr 2013. Drucksache 18/705 vom 06.10.2014.

- (2015): Antwort der Bundesregierung auf die Kleine Anfrage der Abgeordneten Ulla Jelpke, Jan Korte, Sevim Dağdelen, weiterer Abgeordneter und der Fraktion DIE LINKE. Ergänzende Informationen zur Asylstatistik für das dritte Quartal 2015. Drucksache 18/6860 vom 05.01.2016.

Eaton, Jonah (2013): »The Internal Protection Alternative Under European Union Law: Examining the Recast Qualification Directive«, in: International Journal of Refugee Law 24, S. 765-792.

Evans, Tony (2011): »Professionals, Managers and Discretion: Critiquing StreetLevel Bureaucracy«, in: British Journal of Social Work 41(2), S. 368-386.

European Migration Network (2012): Ad-Hoc Query on early legal advice for asylum seekers. Requested by IE EMN NCP on $24^{\text {th }}$ August 2012, Compilation produced on $19^{\text {th }}$ November 2012.

Feldman, Martha S./Pentland, Brian T. (2003): »Reconceptualizing Organizational Routines as a Source of Flexibility and Change«, in: Administrative Science Quarterly 48, S. 94-118.

Fischer-Lescano, Andreas/Christensen, Ralph (2005): »Auctoritatis Interpositio.

Die Dekonstruktion des Dezisionismus durch die Systemtheorie«, in: Der Staat 44(2), S. 213-242.

Furre, Harald/Gustafsson, Mariana S./Quarles, Jannecke/Viblemo, Tor E. (2010): Organisering teller, ressurser avgjør. Asylsaksbehandlingen i norsk 
utlendingsforvaltning $\mathrm{i}$ et komparativt perspektiv, Kristiansand: Oxford Research.

Gibb, Robert/Good, Anthony (2013): »Do the Facts Speak for Themselves? Country of Origin Information in French and British Refugee Status Determination Procedures«, in: International Journal of Refugee Law 25, S. 291-322.

- (2014): »Interpretation, translation and intercultural communication in refugee status determination«, in: Language and intercultural communication 14, S. 385-399.

Guild, Elspeth (2006): »The Europeanisation of Europe's Asylum Policy«, in: International Journal of Refugee Law 18, S. 630-651.

Hils, Sylvia/Streb, Sebastian (2010): Vom Staatsdiener zum Dienstleister? Veränderungen öffentlicher Beschäftigungssysteme in Deutschland, Großbritannien, Frankreich und Schweden, TranState Working Papers 111.

Holzer, Thomas/Schneider, Gerald (2002): Asylpolitik auf Abwegen. Nationalstaatliche und europäische Reaktionen auf die Globalisierung der Flüchtlingsströme, Opladen: Leske + Budrich.

Hupe, Peter/Hill, Michael (2007): »Street-level bureaucracy and public accountability«, in: Public Administration 85(2), S. 279-299.

Hupe, Peter/van Kooten, Eva (2015): »First-line supervisors as gate-keepers: rule processing by head teachers«, in: Peter Hupe/Michael Hill/Aurèlien Buffat (Hg.), Understanding Street-level Bureaucracy, Bristol/Chicago: Policy Press, S. 227-242.

Ippolito, Francesca/Velluti, Samantha (2011): »The Recast Process of the EU Asylum System: A Balancing Act Between Efficiency and Fairness «, in: Refugee Survey Quarterly 30, S. 24-62.

Jahn, Detlef (2009): »Das politische System Schwedens«, in: Wolfgang Ismayr (Hg.), Die politischen Systeme Westeuropas, Wiesbaden: VS Verlag, S. 107-150.

Jubany, Olga (2011): »Constructing truths in a culture of disbelief: Understanding asylum screening from within«, in: International Sociology 26, S. 74-94.

Kaunert, Christian/Léonard, Sarah (2012): »The European Union Asylum Policy after the Treaty of Lisbon and the Stockholm Programme: Towards Supranational Governance in a Common Area of Protection?«, in: Refugee Survey Quarterly 31, S. 1-20.

Kreienbrink, Axel (2013): »60 Jahre Bundesamt für Migration und Flüchtlinge im Kontext der deutschen Migrationspolitik«, in: Zeitschrift für Ausländerrecht und Ausländerpolitik 33, S. 397-448. 
Lahusen, Christian (2016): »Auf dem Weg zu einem europäischen Verwaltungsfeld? Zur Soziologie der Bürokratisierung Europas am Beispiel des Asyls«, in: Berliner Journal für Soziologie 26, S. 109-133.

Lambert, Hélène (2009): »Transnational judicial dialogue, harmonization and the Common European Asylum System«, in: International and Comparative Law Quarterly 58, S. 519-544.

Liebig, Brigitte (2007): »)Tacit Knowledge< und Management. Ein wissenssoziologischer Beitrag zur qualitativen Organisationskulturforschung «, in: Ralf Bohnsack/Iris Nentwig-Gesemann/Arnd-Michael Nohl (Hg.), Die dokumentarische Methode und ihre Forschungspraxis, Opladen: Budrich, S. 147-165.

Lipsky, Michael (2010): Street-level bureaucracy. Dilemmas of the individual in public services, 30th anniversary expanded edition, New York: Russell Sage Foundation.

Meyer, John W. (2005): Weltkultur. Wie die westlichen Prinzipien die Welt durchdringen, herausgegeben von Georg Krücken, aus dem Amerikanischen von Barbara Kuchler, Frankfurt am Main: Suhrkamp.

Miettinen, R./Virkkunnen, J. (2005): »Epistemic Objects, Artefacts and Organizational Change«, in: Organization 12, S. 437-456.

Neumayer, Eric (2005): »Asylum recognition rates in Western Europe - their determinants, variation and lack of convergence [online]«, in: London: LSE Research Online.

Nohl, Arnd-Michael (2012): Interview und dokumentarische Methode. Anleitungen für die Forschungspraxis (= Qualitative Sozialforschung), Wiesbaden: VS Verlag für Sozialwissenschaften.

Parusel, Bernd (2015a): »Solidarity and fairness in the common European asylum system - failure or progress?«, in: Migration Letters 12, S. 124-136.

- (2015b): Einwanderung in Schweden: Zukünftige Herausforderungen. Bundeszentrale für Politische Bildung, http://www.bpb.de/gesellschaft/migration/ laenderprofile/57862/herausforderungen vom 21.03.2016.

Pöllabauer, Sonja (2007): »Interpreting in asylum hearings«, in: Cecilia Wadensjö/Birgitta Englund Dimitrova/Anna-Lena Nilsson (Hg.), The Critical Link 4, Amsterdam: John Benjamins Publishing Company, S. 39-52.

Probst, Johanna (2012): Instruire la demande d'asile. Étude comparative du processus décisionnel au sein de l'administration allemande et francaise, Unveröffentlichte Dissertation, Université de Strasbourg/Philipps-Universität Marburg.

Rosset, Damian/Maia Liodden, Tone (2015): »The Eritrea report- Symbolic uses of expert information in asylum politics «, in: Oxford Monitor of Forced Migration 5, S. 26-32. 
Scheffer, Thomas (1997): »Dolmetschen als Darstellungsproblem. Eine ethnographische Studie zur Rolle der Dolmetscher in Asylanhörungen«, in: Zeitschrift für Soziologie 26, S. 159-180.

- (1998): »Übergänge von Wort und Schrift: zur Genese und Gestaltung von Anhörungsprotokollen im Asylverfahren«, in: Zeitschrift für Rechtssoziologie 20, S. 230-265.

— (2003): »Kritik der Urteilskraft - wie die Asylprüfung Unentscheidbares in Entscheidbares überführt«, in: Jochen Oltmer (Hg.), Migration steuern und verwalten. Deutschland vom späten 19. Jahrhundert bis zur Gegenwart, Göttingen: V \& R Unipress, S. 423-458.

Schittenhelm, Karin (2015): »Asylsuchende im Blickfeld der Behörde. Explizites und implizites Wissen in der Herstellung von Asylbescheiden in Deutschland «, in: Soziale Probleme 26(2), S. 137-150.

Schneider, Jan (2012): Die Organisation der Asyl- und Zuwanderungspolitik in Deutschland. Studie der deutschen nationalen Kontaktstelle für das Europäische Migrationsnetzwerk (EMN), 2. überarbeitete und aktualisierte Auflage, Nürnberg: Bundesamt für Migration und Flüchtlinge.

Schoultz, Isabel (2014): »Seeking Asylum and Residence Permits in Sweden: Denial, Acknowledgement, and Bureaucratic Legitimacy«, in: Critical Criminology 22, S. 219-235.

Spijkerboer, Thomas P. (Hg.) (2013): Fleeing homophobia. Sexual orientation, gender identity and asylum, Abingdon/Oxon: Routledge.

Staffans, Ida (2008): »Evidentiary Standards of Inquisitorial versus Adversarial Asylum Procedures in the Light of Harmonization«, in: European Public Law 14, S. 615-641.

Strauss, Anselm/Corbin, Juliet (1990): Basics of Qualitative Research. Grounded Theory Procedures and Techniques, Newbury Park/London/New Delhi: SAGE Publications.

Toshkov, Dimiter/Haan, Laura de (2013): »The Europeanization of asylum policy. An assessment of the EU impact on asylum applications and recognitions rates«, in: Journal of European Public Policy 20, S. 661-683.

Vink, Maarten P./Meijerink, Frits (2003): »Asylum Applications and Recognition Rates in EU Member States 1982-2001: A Quantitative Analysis«, in: Journal of Refugee Studies 16, S. 297-315.

Weick, Karl E. (1998): »Introductory Essay-Improvisation as a Mindset for Organizational Analysis«, in: Organization Science 9, S. 543-555.

Wettergren, Åsa (2013): A Normal Life: Reception of Asylum Seekers in an Italian and a Swedish Region, Gothenburg: Gothenburg University. 
Wettergren, Åsa/Wikström, Hanna (2014): »Who Is a Refugee? Political Subjectivity and the Categorisation of Somali Asylum Seekers in Sweden «, in: Journal of Ethnic and Migration Studies 40, S. 566-583.

Yates, Jacqueline (2000): »Sweden«, in: J. A. Chandler (Hg.), Comparative public administration, London: Routledge, S. 148-172.

\section{Internetquellen}

Bundesamt für Migration und Flüchtlinge (2015a): Das Bundesamt in Zahlen 2014. Asyl, Migration und Integration, Nürnberg, www.bamf.de/Shared Docs/Anlagen/DE/Publikationen/Broschueren/bundesamt-in-zahlen-2014. pdf?_blob=publicationFile vom 03.03.2016.

— (2015b): Schlüsselzahlen Asyl 1.Halbjahr 2015, www.bamf.de/SharedDocs/ Anlagen/DE/Publikationen/Flyer/flyer-schluesselzahlen-asyl-halbjahr-2015. pdf?_blob=publicationFile vom 03.03.2016.

EASO (2014a): EASO-SCHULUNGSPROGRAMM, https://www.easo.europa. eu/sites/default/files/public/BZ0413152DEC.pdf vom 30.05.2014.

- (2014b): EASO Practical Guide: Personal interview, https://www.easo. europa.eu/practical-tools vom 04.06.2015.

- (2015a): Annual Training Report 2014, https://easo.europa.eu/wpcontent/uploads/EASO_2014_Annual-Training-Report.pdf vom 08.01.2016.

- (2015b): Newsletter - November/December 2015, https://www.easo.europa. eu/newsletter vom 08.01.2016.

- (2016): Annual Training Report 2015, https://www.easo.europa.eu/informa tion-analysis/annual-report vom 01.06.2016.

EUROSTAT (02.03.16): First instance decisions on applications by citizenship, age and sex. Annual aggregated data (rounded) [migr_asydcfsta], http:// ec.europa.eu/eurostat/web/asylum-and-managed-migration/data/database vom 03.03.16.

Migrationsverket (o.J.): The Mission of the Migration Agency, http://www.mig rationsverket.se/English/About-the-Migration-Agency/Our-mission.html vom 04.06.2015.

— (2014): Årsredovisning, http://www.migrationsverket.se/download/18.39a9cd 9514a346077212ead/1424702424160/\%C3\%85rsredovisning+2014.pdf vom 04.06.2015.

— (2015): EMN Policy report 2014 SWEDEN, Norrköping vom 25.02.2016. 
Svensson, Anna H./Mattsson, Pontus/Magnusson, Orjan (2016): Ygeman: Uppemot 80.000 asylsökande kan utvisas, http://www.svt.se/nyheter/inrikes/ tiotusentals-asylsokande-ska-avvisas vom 21.03.2016. 



\section{Entscheiden über Asyl}

Organisationssoziologische Überlegungen

zum Zusammenspiel von Formalität und Informalität

im österreichischen Asyl-Verwaltungsverfahren

JULIA DAHLVIK

\section{Einleitung}

2014 wurden EU-weit mehr als 625.000 Asylanträge gestellt, in Österreich haben über 28.000 Personen internationalen Schutz gesucht (vgl. Bitoulas 2015). Was geschieht mit diesen Anträgen, wie, von wem und unter welchen Bedingungen werden sie bearbeitet und entschieden? Mit diesem Thema befasst sich der vorliegende Beitrag. Auf der Grundlage ethnographischer Forschung im ehemaligen Bundesasylamt (seit 2014 Bundesamt für Fremdenwesen und Asyl), einer Einrichtung des österreichischen Innenministeriums, wird die institutionelle Praxis der administrativen Bearbeitung von Asylanträgen untersucht.

Gesellschaftlichen, politischen und rechtlichen Entwicklungen folgend ist in den letzten zehn bis zwanzig Jahren ein Forschungszweig entstanden, der sich aus unterschiedlichen disziplinären Perspektiven genau diesen Fragestellungen widmet. Wer sind die EntscheiderInnen, welche sozialen Praxen lassen sich in ihrem Arbeitsalltag beobachten, wie sind institutionelle Prozesse geordnet und strukturell verankert? Während Untersuchungen der Anthropologie, Politik- und Rechtswissenschaft wichtige Beiträge leisten, so fehlt bislang eine organisationssoziologische Auseinandersetzung mit der Asylverwaltung. Zudem wird in den vorhandenen Studien selten die theoretische Brücke zu sozialem Handeln in Organisationen über den speziellen Kontext hinaus geschlagen. 
An dieser Stelle setzt der vorliegende Beitrag an und untersucht, welchen Beitrag ein organisationssoziologischer Ansatz zur Erklärung der beobachteten Phänomene leisten kann, insbesondere unter Berücksichtigung eines strukturations- und praxistheoretischen Zugangs. Das interessierende Phänomen umfasst in diesem Fall die auf Basis des empirischen Datenmaterials identifizierten strukturellen Dilemmata in der Bearbeitung von Asylanträgen sowie die damit verknüpften sozialen Praxen im Alltag der EntscheiderInnen. Denn die Ausgangshypothese des Forschungsprojekts, auf dem der vorliegende Beitrag basiert, lautet, dass gegensätzliche rechtliche, administrative und humanitäre Logiken EntscheiderInnen im Asylverfahren mit strukturellen Dilemmata konfrontieren. Ein Blick in und durch den Staatsapparat anhand einer Behörde wie dem Bundesasylamt erlaubt es, das professionelle Alltagshandeln der EntscheiderInnen im institutionellen Kontext zu erforschen und somit das staatliche Handeln auf individueller und organisationaler Ebene sichtbar zu machen.

In diesem Beitrag werden zunächst der Stand der Forschung, das Erkenntnisinteresse der Untersuchung sowie der method(olog)ische Zugang erläutert. Anschließend wird diskutiert, wie strukturations- und praxistheoretische Ansätze in der soziologischen Organisations- und Arbeitsforschung nutzbar gemacht werden können. Ein Fokus liegt dabei auf der sozialen Praxis in Organisationen im Zusammenspiel zwischen Formalität ${ }^{1}$ und Informalität. Dieses Zusammenspiel und gleichzeitig Spannungsfeld wird folglich anhand der vier im Kontext der Asylverwaltung identifizierten strukturellen Dilemmata illustriert. Im Rahmen dessen werden unterschiedliche Prozesse der Formalisierung im administrativen Asylverfahren erläutert, wobei der sozialen Konstruktion von Fakten besondere Bedeutung zukommt. Der Beitrag schließt mit einer Conclusio zur Strukturation des organisationalen Geschehens im Kontext von Formalität und Informalität im Bundesasylamt ab.

\section{Stand der Forschung, Erkenntnisinteresse und Methodik}

Dieser Beitrag lässt sich in der Forschungstradition der street-level bureaucracy verorten, die um 1980 mit Prottas, Lipsky und Brown ihren Anfang nahm und sich bei der Untersuchung der Strukturen von Arbeit in bürokratischen Organisationen schwerpunktmäßig mit folgenden Themen befasst: AkteurInnen und ihre

1 Formal bzw. formell wird im vorliegenden Text im Sinne von >einer offiziell festgelegten Ordnung entsprechend $/$ verwendet. 
Position in der Organisation (frontline workers), ihr Gegenüber (KlientInnen und BürgerInnen), das Ermessen sowie die notwendige Eigenverantwortlichkeit, über die sie verfügen, und die Politikgestaltungsmacht, die sie durch ihre Position, ihr Ermessen und ihre relative Eigenverantwortlichkeit erlangen (vgl. MaynardMoody/Portillo 2010: 255). Insbesondere dem Konzept des Ermessens innerhalb der street-level bureaucracy widmen sich viele AutorInnen (vgl. Hupe 2013).

Die soziologisch-empirische Erforschung des Arbeitsalltags in bürokratischen Organisationen ist im deutschsprachigen Raum im Allgemeinen nicht sehr ausgeprägt. Zwar beschäftigt sich die Organisationssoziologie auch mit staatlichen Institutionen, jedoch fand die durch Mayntz (1997) etablierte Soziologie der öffentlichen Verwaltung keine große Verbreitung. Während in anderen Ländern, insbesondere in Frankreich und den USA, Forschung im Bereich der öffentlichen Verwaltung Tradition hat, ist die soziologische Auseinandersetzung mit den relevanten Strukturen und Prozessen im deutschsprachigen Raum bei Weitem nicht erschöpft. Im Bereich der politischen Soziologie finden sich jedoch beispielsweise Arbeiten zur Bürokratieforschung (vgl. Grunow 2009), zu administrativen Kulturen (vgl. Grießler 2003) und zum Lernen in Verwaltungseinheiten (vgl. Biegelbauer 2013). Zur Erforschung des Arbeitsalltags sind unter anderem Beiträge zur Bedeutung von Emotionen in der Verwaltung zu nennen (vgl. Sondermann/Englert 2013; Behrend 2013).

Die Verwaltung im Bereich des Asylwesens wurde bereits in einigen europäischen Ländern ethnographisch näher untersucht (vgl. Scheffer 2001; Spire 2007; Jubany 2011; Probst 2013). Während diese Studien internationale Vergleiche ermöglichen (bei denen große Ähnlichkeiten feststellbar sind), fehlte bislang eine solche institutionenbezogene Untersuchung in Österreich. Es findet zwar Forschung zur österreichischen Asylpolitik statt (vgl. Rosenberger 2010), es werden rechtswissenschaftliche Analysen der Gesetze durchgeführt (vgl. Schumacher/Peyrl/Neugschwendtner 2015) und öffentlich zugängliche Medien diskursanalytisch untersucht (vgl. Arnim-Ellissen 2012). Auf individueller Ebene werden u.a. das Rechtsbewusstsein und die »Asylwirklichkeit« von Asylsuchenden erforscht (vgl. Fritsche 2012). Wie sieht es aber mit jenen Prozessen aus, die gewissermaßen hinter verschlossenen Türen, das heißt nicht öffentlich, ablaufen? Bisherige Forschungen haben sich im österreichischen Kontext primär mit der Kommunikationssituation in Asyleinvernahmen befasst (vgl. Pöllabauer 2005; Plutzar 2009; Auzinger 2012; Ammer et al. 2013). Darüber hinaus blieb das Handeln der Institution beziehungsweise der EntscheiderInnen in diesem Verwaltungsverfahren bislang unerforscht. Während ein öffentlicher Diskurs 
über Transparenz $z^{2}$ in staatlichen Institutionen existiert, sind verwaltungsrechtliche Verfahren tatsächlich häufig durch Intransparenz geprägt, sowohl für die Beteiligten als auch für Außenstehende. Mangelndes Verständnis (von AntragstellerInnen aber auch der Öffentlichkeit) für administrative Handlungen und Entscheidungen kann zumindest teilweise auf die mangelnde Nachvollziehbarkeit institutioneller Prozesse, Verantwortlichkeiten und Handlungsketten zurückgeführt werden.

Bislang fehlt also nicht nur eine empirische Untersuchung der sozialen Praxis im organisationalen Kontext, sondern auch eine theoretische Auseinandersetzung mit institutionellem Handeln im Bereich der Asylverwaltung. Der vorliegende Artikel versucht hier einen Beitrag zu leisten, indem folgende Fragestellungen untersucht werden: Wie kann die soziale Praxis der EntscheiderInnen im Umgang mit den im Rahmen der Untersuchung identifizierten Dilemmata im organisationalen Kontext erklärt werden? Welchen Beitrag können organisationsbezogene strukturations- und praxistheoretische Ansätze zur Erklärung dieser sozialen Praktiken liefern? Das Erkenntnisinteresse gilt daher den Wechselwirkungen zwischen der alltäglichen Praxis der ReferentInnen im bürokratischen Entscheiden über Asylanträge (auch im Umgang mit Asylsuchenden) und den strukturellen, organisationalen Bedingungen, die dieses Handeln der AkteurInnen ermöglichen, einschränken, prägen.

Ein Zugang, der es ermöglicht, diese Fragestellungen empirisch zu bearbeiten, ist jener der institutionellen Ethnographie (vgl. Smith 2006). Gesellschaftlich relevante Prozesse wie jener der »Migrationsverwaltung « können im Rahmen einer solchen Feldforschung in einem Dialog mit den EntscheiderInnen und nicht bloß über die EntscheiderInnen untersucht werden. In der hier vorgestellten Studie wurde eine Kombination unterschiedlicher qualitativer Methoden angewandt, um Erkenntnisse über die soziale Praxis der EntscheiderInnen zu gewinnen und so einer Mystifizierung des bürokratischen Alltags entgegenzuwirken. Durch Interviews konnten beispielsweise die Beziehungen (einschließlich Auffassungen, Zuschreibungen und Erwartungen) der ReferentInnen zu den Asylsuchenden, aber auch zu anderen AkteurInnen, ebenso wie Einstellungen zur Organisation und zur eigenen Tätigkeit erforscht werden. Teilnehmende Beobachtung hingegen erlaubte einerseits das Eintauchen in den bürokratischen Alltag und das Kennenlernen von Routinen (zum Beispiel des Schreibens) und andererseits die Untersuchung von Interaktions- und Kommunikationsprozessen innerhalb der Organisation wie auch in der Begegnung mit Asylsuchenden in den so-

2 Siehe dazu die Initiative zur Schaffung eines Transparenzgesetzes, www.transparenz gesetz.at vom 20.5.2016. 
genannten Einvernahmen (vgl. Dahlvik 2014). Durch die ergänzende Analyse von Artefakten (insbesondere Asylakten) war es möglich, zusätzliches Wissen über die Funktionsweise und innere Logik der Organisation zu generieren. ${ }^{3}$ Die Analyse der Beobachtungen, Interviews und Artefakte orientierte sich primär an dem Zugang der interpretativen Sozialforschung (vgl. Froschauer/Lueger 2009) und dem Kodierparadigma der Grounded Theory (vgl. Strauss/Corbin 1990).

\section{Organisationales Handeln zwischen Formalität und Informalität: strukturations - und praxistheoretische Ansätze}

Da es ein Ziel dieses Beitrag ist, die beobachtbaren sozialen Praktiken der EntscheiderInnen im Bundesasylamt aus organisationsbezogener strukturations- und praxistheoretischer Perspektive zu erklären, sollen wesentliche Elemente dieser Theorieansätze zunächst kurz erläutert werden.

Folgt man der Theorie der Strukturierung (Giddens 2011[1986]) zur Erklärung sozialen Handelns, so nehmen AkteurInnen in Interaktionen immer Bezug auf Strukturen, das heißt auf Regeln und Ressourcen. Gleichzeitig werden die strukturellen Charakteristika des Handlungsfeldes durch das strukturierende Handeln der AkteurInnen produziert, wie beispielsweise eine strikte Arbeitsteilung. Indem AkteurInnen auf diese Strukturen rekurrieren, reproduzieren sie diese zugleich. Weiters wird davon ausgegangen, dass kompetente AkteurInnen sich mehr oder weniger reflexiv auf ihre eigene Vergangenheit, Gegenwart und Zukunft beziehen, aber auch auf das Handeln der Anderen und die strukturellen Eigenschaften des Handlungsfeldes. Allerdings können sie die Prozesse der sozialen Reproduktion nie vollständig kontrollieren. Auch als kompetente AkteurInnen handeln sie oft auf der Grundlage von »praktischem «, implizitem Wissen; sie tun also etwas, ohne erklären zu können, wie und warum sie dies tun. Strukturationstheoretische Ansätze der Organisationssoziologie wenden die hier be-

3 Der Großteil der Beobachtungen hat im Rahmen eines kurzzeitigen Praktikums in einer Außenstelle des Bundesasylamts stattgefunden. Insgesamt wurden zwölf Leitfaden-Interviews mit unterschiedlichen Akteuren geführt (mit MitarbeiterInnen einer damaligen Erstaufnahmestelle und einer Außenstelle der Asylbehörde sowie einem Richter des damaligen Asylgerichtshofes). $\mathrm{Zu}$ den analysierten Dokumenten und Artefakten zählen Medienberichte, aber auch allgemein zugängliche institutionelle Schriftstücke sowie drei Akten von Asylsuchenden. Die Datengenerierung hat im Rahmen meines Dissertationsprojekts zwischen 2010 und 2012 stattgefunden. 
schriebene Dualität - das sich wechselseitig konstituierende Zusammenspiel von Struktur und Handeln nicht nur auf Individuen, sondern auf Organisationen als Untersuchungseinheiten an; die Organisation wird also als reflexive Strukturation verstanden (vgl. Ortmann/Sydow/Windeler 2000; Sydow/Wirth 2014).

Die Theorie der sozialen Praxis schließt hier nahtlos an, da sie zu einem wesentlichen Teil auf Giddens' (2011[1986]) Strukturationstheorie sowie Bourdieus' (1972) Theorie der Praxis basiert. Während die Praxistheorie bisher keine abgeschlossene, systematisierte Sozialtheorie darstellt, legt Reckwitz (2003: 282) eine programmatische Skizze vor, in der er folgende drei Grundannahmen definiert: »eine simplizite<, >informelle< Logik der Praxis und Verankerung des Sozialen im praktischen Wissen und `Können〈; eine `Materialität` sozialer Praktiken in ihrer Abhängigkeit von Körpern und Artefakten; schließlich ein Spannungsfeld von Routinisiertheit und systematisch begründbarer Unberechenbarkeit von Praktiken«. Die Praxistheorie will aus einer kulturtheoretischen, sozialkonstruktivistischen Perspektive das praktische Wissen, das in einer sozialen Praktik mobilisiert wird, rekonstruieren. Dieses ist zum einen ein Wissen im Sinne eines interpretativen Verstehens, das heißt einer Routine der Bedeutungszuschreibung zu Gegenständen, Personen oder Abstraktem. Zum anderen geht es dabei um methodisches Wissen in Form "scriptförmige[r] Prozeduren «, an denen AkteurInnen ihr Handeln orientieren (ebd.: 292). Ein drittes Element des praktischen Wissens stellt das "motivational-emotionale Wissen « (ebd.) dar, welches sich darauf bezieht, dass AkteurInnen beispielsweise einen impliziten Sinn dafür entwickeln, worum es ihnen bei einer Praxis geht oder was für sie unvorstellbar wäre. Diese Wissensformen werden in der Praxistheorie dabei nicht als universal, sondern als historisch-spezifisch, als ein letztlich kontingentes »local knowledge « (Geertz 1983) verstanden. Auch dadurch ist sie mit der Grundannahme einer informellen, impliziten Logik des Sozialen und des Handelns eng verknüpft. Ein arbeits- und organisationssoziologisch relevantes Anwendungsfeld der Theorie der sozialen Praxis ist die Wissenschafts- und Technikforschung: Hier wird ein heterogener Komplex von informellen Verhaltensroutinen »at work « rekonstruiert, welche wiederum von einem impliziten Hintergrundwissen gestützt werden (vgl. Reckwitz 2003: 284). Diesem Ziel verschreibt sich auch der vorliegende Beitrag im Kontext der administrativen Bearbeitung von Asylanträgen.

In der Organisationsforschung bilden praxeologische Ansätze einen Gegenpol zu klassischen ökonomischen Theorien wie Rational Choice oder postWeberscher formaler Rationalität in bürokratisch-hierarchischen Organisationen. Anstatt rationalistischer Entscheidungstheorien stehen rekursive Verhaltensroutinen im Organisationsalltag im Mittelpunkt von Forschungen, die sich seit den 
1980er Jahren Giddens' Strukurationstheorie aus Organisationsperspektive widmen. Zentrale Themen sind beispielsweise Entscheidungen unter Ungewissheit und damit zusammenhängend die Rolle von Daumenregeln und Vertrauensbeziehungen. AkteurInnen werden dabei nicht normativ idealisiert als Individuen, die expliziten institutionellen Normen folgen. Stattdessen geht man davon aus, dass informelle Praktiken den Alltag in der Organisation strukturieren, die auch regelmäßig den offiziellen Gesetzen zuwiderlaufen können (vgl. ebd.: 285)

Im Kontext der organisationalen Arbeit sind Erfahrungswissen und situatives Handeln also nicht mehr nur als Abweichung zu erfassen, sondern müssen in einer eigenen Systematik analysiert werden. Der Fokus auf situatives Handeln bedeutet auch, dass das Wie des Arbeitshandelns in den Mittelpunkt gerückt wird. ${ }^{4}$ In der soziologischen Forschung wurde in diesem Zusammenhang das Konzept des »erfahrungsgeleitet-subjektivierenden Handelns« (Böhle/Voß/ Wachtler 2010: 160) entwickelt, das einerseits auf das Erfahrung-Machen als Grundlage des Erwerbs von Wissen hinweist und andererseits auf die kognitive und handlungspraktische Deutung von subjektiven Faktoren wie Gefühle und Empfinden Bezug nimmt. Hinsichtlich der Formen informeller Kooperation wurde auch das damit zusammenhängende Konzept der erfahrungsgeleitetsubjektivierenden Kooperation entwickelt, das im Gegensatz zu »planungsbezogen-objektivierender Kooperation« steht (zum Beispiel Kooperation im Rahmen von Besprechungen) (vgl. Böhle/Bolte 2002; Bolte/Porschen 2006).

Die erfahrungsgeleitet-subjektivierende Kooperation weist folgende Merkmale auf: »Der Anlass, der Zeitpunkt und die beteiligten Partner ergeben sich situativ je nach Problemlage; die Verständigung erfolgt auf der Grundlage gemeinsamer Erfahrungen und mit Hilfe von Gegenständen; und die Beziehung zwischen den Kooperationspartnern basiert auf einer wechselseitigen (arbeitsbezogenen) Vertrautheit. « (Böhle/Voß/Wachtler 2010: 164) Da sich solche Formen der Kooperation und Kommunikation nicht oder nur begrenzt formalisieren lassen, ergibt sich ein strukturell informeller Charakter von sozialen Praktiken und Prozessen. In Bezug auf das Beispiel der Kooperation liegt der Unterschied zwischen formellen und informellen Formen selbstgesteuerter Kooperation vor allem in der Art des kooperativen Handelns und den daraus resultierenden Möglichkeiten und Begrenzungen der Formalisierung (vgl. Böhle/Voß/Wachtler 2010).

4 Die meist ethnographischen »Work-Place-Studies« im anglo-amerikanischen Raum haben maßgeblich zur Entwicklung des Konzepts des situativen Handelns beigetragen (vgl. Knoblauch/Heath 1999). 
Die Unterscheidung zwischen Formalität und Informalität zählt zu den klassischen Unterscheidungen der Organisationsforschung. 1955 etwa formulierte der Soziologe Peter Blau die Einsicht, dass für das Funktionieren einer Organisation nicht nur formale Strukturen bedeutsam sind, sondern ebenso informale (vgl. Blau 1955). Auch Erhard Friedberg (1995) stellte fest, dass Regelabweichungen für das Funktionieren einer Organisation eine wesentliche Rolle spielen. $^{5}$

Nach Froschauer und Lueger (2015) stellen Formalität und Informalität zwei Ordnungsformen dar, die eng aufeinander bezogen sind, jedoch unterschiedliche organisationale Bereiche regeln. Die AutorInnen gehen davon aus, dass die Informalität letztlich die Formalität dominiert. Auch in der behördlichen Praxis der Asylantragsbearbeitung kann festgestellt werden, dass die informellen $\gg$ Pole $<$ der (im Folgenden beschriebenen) Dilemmata den Alltag eher dominieren als die formellen. Die formalen Anforderungen - Normierung, Individualisierung, Entscheidung und Verantwortung - bilden den Rahmen der Handlung. Formalität schafft also einen stabilen Rahmen für soziale Prozesse, welche durch Informalität geregelt werden, »ein erwartungsstabiles Gerüst mit allgemeinen Regeln, das zugleich als rituelle Fassade dient.« (Ebd.: 204) Dieser abstrakte Rahmen dient auch dazu, »Entscheidungen extern über eine verbindliche Verfahrensregelung « zu legitimieren (ebd.: 205). Um die Entscheidung offiziell und formell brauchbar zu machen, muss sie in diesen Rahmen der Formalität eingepasst werden. Darüber hinaus bietet Formalisierung eine einklagbare Verweisungsstruktur und erlaubt es gleichzeitig, Verantwortung zu externalisieren. Letzteres ist auch in der Asylverwaltung eine gängige Praxis, wie im nachfolgenden Abschnitt zu sehen sein wird.

»Informelle Interaktionsordnungen « (ebd.) hingegen basieren auf Vertrauensbeziehungen und erwartungsstabilem Handeln. Sie ermöglichen Flexibilität und Heterogenität - beispielsweise unterschiedliche individuelle Zugänge der EntscheiderInnen - machen jedoch auch Entscheidungen intransparent. Festzuhalten ist auch, dass Informalität Erfahrung und implizites, häufig nicht explizierbares, Wissen erfordert (vgl. ebd.). So weist beispielsweise das Prinzip des »learning by doing « im Rahmen der Einschulung von EntscheiderInnen auf die Relevanz von Erfahrung und implizitem Wissen in der Bearbeitung von Asylan-

5 Auch außerhalb des organisationalen Kontexts befasst sich die Soziologie mit diesem Thema, so zum Beispiel mit informell eingebetteter Formalität, formell eingebetteter Informalität, der Interaktion zwischen Formalität und Informalität sowie dem Entstehen und der Transformation von Formalität und Informalität (vgl. Mica/ Winczorek/Wisniewski 2015). 
trägen hin. Auch die Bedeutung der Sozialisation durch die Organisationskultur wird in diesem Kontext ersichtlich. Denn EntscheiderInnen orientieren sich bei der Entwicklung von Strategien, beispielsweise betreffend den Umgang mit Asylsuchenden in der Einvernahme, nicht nur an administrativen Vorgaben, sondern auch an der Praxis ihrer KollegInnen. Froschauer und Lueger (ebd.: 206) zeigen, dass »die explizit geltenden Regeln [...] in vielen Fällen den Handelnden entweder gar nicht genau bekannt sind oder dass dieses Wissen ungleich verteilt ist«. So lässt sich auch im Bundesasylamt beobachten, dass vor allem jene EntscheiderInnen, die über keine juristische Ausbildung verfügen, die rechtlichen Grundlagen ihres Handelns nur ansatzweise kennen. »In der Praxis sind es der Glaube an bestimmte formale Strukturen und die Legitimität dieser imaginierten Ordnung sowie die kollektive Orientierung an dieser Ordnungsvorstellung, die diese mit dem Alltagshandeln verknüpft.«(Ebd.)

Sowohl Formalität als auch Informalität leisten ihren, auch in der Verwaltung relevanten, Beitrag zur Komplexitätsreduktion. Während formelle Strukturen Handlungsspielräume standardisieren und so ein »Korsett für Zulässiges« (ebd.: 204) darstellen, können auf informeller Ebene häufig Entscheidungen getroffen werden, ohne Rücksprachen treffen zu müssen. Die im Folgenden beschriebenen sozialen Praxen weisen ebenfalls auf Prozesse der Komplexitätsreduktion hin, zum Beispiel durch die Konstruktion von homogenisierten Gruppen von Asylsuchenden oder >Fällen` unter EntscheiderInnen, die nicht auf rechtlichen Kategorien basieren.

Schließlich lassen sich aber soziale Praxen nicht immer eindeutig verorten und Vieles findet in einer nicht näher bestimmbaren Grauzone statt, das heißt auch in den »Zonen tolerierter Differenz« von Regelanwendung (Ortmann 2003), irgendwo zwischen Formalität und Informalität. ${ }^{6}$ Denn die Abweichung von Formalität ist nicht in jedem Fall klar identifizierbar, sondern unterliegt der Interpretation, ebenso wie formale Regeln einer Auslegung bedürfen, die nicht immer bis ins letzte Detail formal geregelt ist (vgl. Froschauer/Lueger 2015: 209).

6 Stinchcombe (2001) verwendet beispielsweise das Konzept der »informally embedded formality«. 


\section{Vier Dilemmata im Spannungsfeld von Formalität und Informalität}

Der Fokus auf die sozialen Praktiken und Prozesse innerhalb der Asylverwaltung erlaubt es, alltägliche Routinen und Handlungsstrategien der AkteurInnen zu beleuchten. Dazu zählt auch die Untersuchung von Spannungen und Widersprüchlichkeiten im Arbeitsalltag. Bereits um 1980 befassten sich insbesondere Lipsky (2010[1980]) und Prottas (1979) in ihren Studien zur street-level bureaucracy mit den »Dilemmata des Individuums im öffentlichen Dienst«. Viele dieser Dilemmata sind heute in der öffentlichen Verwaltung ebenso präsent wie damals, und sie sind auch im Bereich der Asylverwaltung anzutreffen.

Auf Basis der Analyse der Interviews, Beobachtungen und Artefakte lassen sich vier ineinander verschränkte Spannungsfelder identifizieren, die den Arbeitsalltag der EntscheiderInnen charakterisieren: (1) Normierung vs. Handlungsspielraum, (2) Individualisierung vs. Generalisierung, (3) Verantwortung vs. Distanzierung, (4) Eindeutigkeit (bzw. Bestimmtheit) vs. Ungewissheit. Diese strukturellen Dilemmata sind zum Teil jenen sehr ähnlich, die bereits von Prottas (1979) und Lipsky (2010[1980]) mit nicht wesentlich anderen Begriffen beschrieben wurden. Das heißt, dass auch die hier identifizierten Spannungsfelder prinzipiell für Verwaltungshandeln auf street-level-Ebene verallgemeinert werden können. Dennoch treten insbesondere die zwei letzteren Dilemmata im Asylverfahren verstärkt zutage, denn einerseits steht in kaum einem anderen Verfahren das Leben eines Menschen auf dem Spiel (Verantwortung) und andererseits sind in den meisten Fällen kaum Beweise vorhanden (Ungewissheit).

Betrachtet man nun die hier beschriebenen Spannungsfelder und damit verbundene soziale Praktiken aus organisationssoziologischer Perspektive, so lassen sich diese auch als funktionale Koexistenz von Formalität und Informalität betrachten. Rechtliche und administrative Normen verlangen und produzieren Formalität in Form von Normierung, Individualisierung, Eindeutigkeit (in Form einer Entscheidung) und Verantwortung. Gleichzeitig ist der Arbeitsalltag in der Behörde durch die Informalität sozialer Praktiken und Prozesse geprägt und zwar in Form von Handlungsspielraum, Ungewissheit, Generalisierung und Distanzierung. Im Folgenden sollen die vier Spannungsfelder und damit zusammenhängenden sozialen Praktiken kurz skizziert werden. Dabei wird auch ersichtlich, dass Informalität und Formalität unterschiedliche Bereiche des organisationalen Handelns regeln und so einander ergänzen.

Formal definieren rechtliche und administrative Normen den Arbeitsalltag der EntscheiderInnen. Gleichzeitig ist bekannt, dass sie bei deren Umsetzung im Rahmen der Bearbeitung eines Asylantrages über einen bestimmten Ermessens- 
und Handlungsspielraum verfügen, selbst wenn die Entscheidung letztlich rechtlichen, administrativen und politischen Vorgaben entsprechen muss. Dieses stellt ein klassisches Spannungsfeld der street-level bureaucracy dar. Das folgende metaphorische Zitat illustriert, dass ihnen der Weg - also das Wie - zur Entscheidung mehr oder weniger frei steht. ${ }^{7}$

»Es gibt ein gewisses Grundschema, das ist für jeden vorgegeben, ist eh klar. Und dann gibt's halt auch einen kleinen Handlungsspielraum oben drauf auf diesen Berg an Vorgaben, wo man sich seinen Weg schon auch nehmen kann, sag ich jetzt mal. Aber es ist nicht so, also es gibt die große Autobahn, du kannst die Spur wählen, sag'ma so, Nebenstraßerl gibt's nicht, der Pannenstreifen, das ist dann, sind die Referatsleiter, [...] man kann auch Spurwechseln.«(R6 1:04)

Der Entscheider beschreibt hier, dass für die Bearbeitung eines Asylantrags bestimmte durch die Organisation vorgegebene Strukturen und Vorgehensweisen bestehen, er aber dennoch Möglichkeiten hat, innerhalb dieser Strukturen flexibel zu agieren. Der Handlungsspielraum der EntscheiderInnen beschränkt sich jedoch nicht auf das rechtliche Ermessen; es schließt beispielsweise auch die Wahl der DolmetscherInnen oder der GutachterInnen ein. Wird überhaupt ein Gutachten in Auftrag gegeben? Was für eines, ein linguistisches, ein medizinisches? Aber auch die Ausführung administrativer und rechtlicher Vorgaben in der direkten Interaktion mit Asylsuchenden im Rahmen von sogenannten Einvernahmen unterliegt dem jeweiligen individuellen Zugang der EntscheiderInnen. Auf welche Art und Weise werden Argumentation und Schlussfolgerungen in das normative Format eines rechtlichen Bescheides gebracht? Individuellen Zugängen und Strategien der EntscheiderInnen kommt in der Bearbeitung von Asylanträgen eine bedeutende Rolle zu. Dies wird auch daran deutlich, dass trotz oder gerade wegen des komplexen normativen Rahmens der Weg von der/dem AnfängerIn bis zur/zum selbstständigen EntscheiderIn primär dem Prinzip des »learning by doing « folgt. Eine Entscheiderin beschreibt, dass sie im Rahmen ihrer Einarbeitung die Einvernahmen verschiedener KollegInnen beobachtet hat, »um den Ablauf mitzukriegen und [...] um sich halt überall was rauszupicken, abzuschauen und dann halt seine eigene Strategie zu entwickeln« (G 1:07). An diesem Beispiel zeigt sich, dass dem impliziten, praktischen Wissen im Arbeitsalltag wesentliche Bedeutung zukommt, denn die Ausbildung zum/zur Entschei-

7 Allerdings hat sich in der Untersuchung gezeigt, dass die jeweils individuelle Herangehensweise der ReferentInnen auch zu unterschiedlichen Entscheidungen führen kann. 
derIn ist nur teilweise formalisiert (etwa durch das Erlangen einer offiziellen Approbation). Mangels formaler Ausbildung wird das Lernen von den Anderen und die Sozialisation in der Organisation zu einem wichtigen Bestandteil auf dem Weg zum/zur EntscheiderIn.

Ein weiteres durchaus klassisches Feld der Widersprüchlichkeiten bilden zeitgleich stattfindende Prozesse der Individualisierung und Generalisierung auf unterschiedlichen Ebenen. Einerseits muss jeder Asylantrag als Einzelfall behandelt und geprüft werden, andererseits führt z.B. die wahrgenommene Wiederholung von Erzählungen bzw. »Vorbringen« von Asylsuchenden zur Konstruktion von Kategorien von AntragstellerInnen. Im Zuge der Generalisierung werden Letztere häufig weniger als Individuen wahrgenommen, sondern vielmehr als Zugehörige homogener, essentialisierter Gruppen (z.B. nach Herkunft oder Religion) gelabelt. So bezweifelt beispielsweise eine Entscheiderin das Verhalten einer Asylsuchenden in einer von der Frau geschilderten Situation, denn »das ist bei tschetschenischen Frauen normalerweise nicht so « (Beobachtungsnotiz 0429). Einerseits sind derartige stereotype Zuschreibungen basierend auf bestimmten Erwartungshaltungen und teils individuellen Maßstäben vorherrschend, andererseits ist es die Aufgabe der EntscheiderInnen, über die Zukunft einer Person mit ihren individuellen Lebensumständen und auf Basis ihrer individuellen Fluchtgeschichte zu urteilen. Der Aspekt der Individualisierung spielt jedoch auch auf einer anderen Ebene ${ }^{8}$ eine Rolle, nämlich hinsichtlich der Arbeitsroutinen der EntscheiderInnen. So bearbeiten letztere Asylanträge nicht im Team, sondern im Alleingang; als Beispiele dienen aber auch die individuelle Handhabe spezifischer Situationen, der Rückgriff auf persönliche Kontakte sowie eigene Erfahrungen der EntscheiderInnen mit Asylsuchenden.

Das dritte Spannungsfeld, das den Arbeitsalltag der EntscheiderInnen charakterisiert, ist jenes zwischen Verantwortung (und Engagement) und Distanzierung (und Banalisierung). Dieses ist insofern weniger klassisch als die anderen beschriebenen Dilemmata, als die Entscheidung im Asylverfahren folgenreicher ist als in den meisten anderen street-level-Kontexten. Ihrer Verantwortung sind sich EntscheiderInnen grundsätzlich zwar bewusst, jedoch wird diese häufig an andere AkteurInnen >weitergeleitet«. So werden meist >die Gesetze` oder >die Politik verantwortlich gemacht und EntscheiderInnen tendieren ganz im Sinne bürokratischer Organisationen dazu, sich lediglich als ausführende Organe zu sehen. Die letztliche Verantwortung wird oftmals auch der Berufungsinstanz ${ }^{9} \mathrm{zu}-$

8 Der Gegenpol ist dementsprechend nicht Generalisierung, sondern das Kollektiv im Sinne einer potentiellen Zusammenarbeit mit KollegInnen.

9 Damals der Asylgerichtshof, heute das Bundesverwaltungsgericht. 
geschanzt. Eine Entscheiderin drückt dies folgendermaßen aus: »Ich sag einmal, wir sind einfach die, also ich finde es so, wir sind die kleinen Indianer, wir machen einmal die Grundarbeit, dass wir das einmal grund-aufbereiten, aber sag'ma die Entscheidung, diese weitsichtige Entscheidung« wird von den RichterInnen gefällt (V 0:20). Als Bedienstete der erstinstanzlichen Behörde beruhigen EntscheiderInnen also häufig ihr Gewissen damit, dass Asylsuchende die Möglichkeit haben, in Berufung zu gehen. Neben dieser Form der Delegation von Verantwortung lassen sich auch andere Praktiken der mentalen Distanzierung und der Banalisierung beobachten, beispielsweise durch den Vergleich mit eigenen Erfahrungen eines bureaucratic encounters bezüglich eines Antrags auf Wohnbauförderung (V 1:49). Weiler (1992) spricht von »banalization of suffering«, wenn in einem bürokratischen System Individuen bzw. menschliche Probleme zu Fällen sowie Kategorien und Lösungen mechanisch angewandt werden.

Nach diesen Ausführungen kann die soziale Praxis der EntscheiderInnen hinsichtlich aller vier Dilemmata dahingehend interpretiert werden, dass die zunächst informellen Praktiken ${ }^{10}$ (Handlungsspielraum, Generalisierung, Distanzierung, Ungewissheit) einen Prozess der Formalisierung durchlaufen, sodass das Ergebnis der Fallbearbeitung lauten kann: Orientierung an den Normen, individuelle Behandlung des Antrags und verantwortungsvolle, definitive Entscheidung.

\section{Prozesse der Formalisierung}

Nachdem nun drei der vier identifizierten Spannungs- und Handlungsfelder im Kontext von Formalität und Informalität in der organisationalen Praxis kursorisch behandelt wurden, soll im nachfolgenden Abschnitt (4.1) das vierte Feld diskutiert werden: der von mir als >Faktisierung < bezeichnete Prozess im Rahmen des Spannungsfeldes >Eindeutigkeit vs. Ungewissheitく. Der oben genannte Transformationsprozess von Informalität zu Formalität kann jedoch nicht nur im Rahmen der Faktisierung beobachtet werden, sondern auch im damit verknüpften Prozess der Verschriftlichung. Der erstgenannte Prozess bezeichnet die Transformation von Information zu Fakten, während der zweite Prozess die Transformation von Gesprochenem zu Geschriebenem umfasst. Bevor wir uns

10 Dabei geht es nicht um eine wertende Beurteilung von Informalität und Formalität, sondern um den Versuch, den mehr oder weniger erzwungenen Wandel von ersterer zu letzterer aufzuzeigen. 
einigen Aspekten des Verschriftlichungsprozesses exkursartig widmen, soll zunächst der Prozess der 〉Faktisierung〈 im Detail behandelt werden.

\section{1 sFaktisierungr - Die strukturell bedingte soziale Konstruktion von Fakten}

Ein Transformationsprozess in Richtung Formalität, den ich als `Faktisierung bezeichne, findet im Rahmen des vierten, noch nicht besprochenen Dilemmas statt. Das Asylverfahren ist ein Rechtsverfahren, in dem meist kaum eindeutige Beweise, sogenannte harte Fakten, für oder gegen die Gefahr von Verfolgung vorhanden sind. Dies erschwert das Entscheiden potentiell, da die für die Entscheidung zuständigen AkteurInnen permanent mit Ungewissheit konfrontiert sind. Es eröffnet sich also ein strukturelles Spannungsfeld, in dem es darum geht, die herrschende Ungewissheit mit einer definitiven Entscheidung, d.h. mit Eindeutigkeit und Bestimmtheit, zu vereinbaren. Im Folgenden wird darauf eingegangen, welche Praktiken EntscheiderInnen entwickeln, um mit der faktischen Unentscheidbarkeit in vielen Fällen umzugehen. Zunächst stellt sich dabei die Frage, welche Informationen benötigt werden, um eine Entscheidung fällen bzw. argumentieren zu können, aber auch wo und wie die als relevant erachteten Informationen zu finden sind. In diesem Kontext soll gezeigt werden, dass der sozialen Konstruktion von Fakten in der Bearbeitung und Entscheidung von Asylanträgen eine wesentliche Bedeutung zukommt.

Eine These, die sich aus der hier vorgestellten Untersuchung ergibt, beschreibt einen Selektions- und Transformationsprozess, der im Rahmen der Bearbeitung von Asylanträgen von EntscheiderInnen und anderen AkteurInnen vollzogen wird: EntscheiderInnen transformieren Information zu einem Faktum, zu etwas, das als objektive Tatsache betrachtet werden kann. Sie sind stetig auf der Suche danach, »was wirklich passiert ist« (S 0:22). Die Befragung der asylsuchenden Person bildet die Basis für den weiteren Verlauf der Antragsbearbeitung. Hier werden Fragen über Fluchtgründe, die private (familiäre, gesundheitliche, politische etc.) Situation im Herkunftsland, aber auch in Österreich gestellt, um Informationen zum $>$ Fall $\prec$ zu erhalten. Diese werden anschließend dahingehend evaluiert, ob sie in der Argumentation für oder wider Asylgewährung als Fakten oder bloße Behauptungen herangezogen werden. Um diese Evaluierung bzw. diesbezügliche erste Annahmen zu unterstützen, werden in der Regel im Anschluss an die Befragung (teils auch vorbereitend) zusätzliche Informationsquellen hinzugezogen. Dass die Suche nach Fakten meist an - sei es rechtlichen, sei es administrativen - Normen orientiert ist, zeigt auch der Rat, den eine Entscheiderin bei der Einarbeitung erhielt: »Nimm den Akt, du solltest wissen, 
was zum Schluss rauskommt. Ja, annähernd wissen.« (S 1:03) Es wird also nur nach solchen Fakten gesucht, die normrelevant sind und eine Entscheidung erlauben, die in das jeweilige >Programm< passt (vgl. Lautmann 2011).

In dieser Recherchephase, die das Ziel hat, Fakten aus Vergangenheit und Gegenwart zu etablieren oder ein zukünftiges Verfolgungsrisiko zu prüfen, geht es allerdings vielmehr um Prozesse der aktiven Konstruktion von Wissen als um passives Entdecken (vgl. Sweeney 2009). Im Asylverfahren besteht meist ein Mangel an Beweisen, der die Argumentation für beide Seiten erschwert. Während Asylsuchende in der Regel kaum identitätsbezogene Dokumente mit sich führen, können sie in manchen Fällen Beweise in Form von Fotos, Briefen oder Zeitungsausschnitten in das Verfahren einbringen. EntscheiderInnen hingegen verfügen über ein breites Spektrum an Möglichkeiten, um an `Fakten`zu gelangen, die als Beweise eingesetzt werden können. Die AkteurInnen der Behörde benötigen autorisierte Informationsquellen, die für eine >objektive` Entscheidung genutzt werden können. Diese sind notwendig, um eine Entscheidung bzw. die damit verbundene Argumentation anhand von Fakten legitimieren zu können.

In der Bearbeitung von Asylanträgen verfügen EntscheiderInnen über ein relativ großes Netzwerk an Informationsquellen. Neben (mehr oder weniger) unabhängigen Fachgutachten stellt die sogenannte Staatendokumentation, ebenfalls eine Einrichtung des Innenministeriums, eine wesentliche Quelle in der Bearbeitung von Asylanträgen dar. Legislative und Jurisdiktion sind freilich ebenso wichtige Orientierungsposten, aber auch individuelle AkteurInnen wie DolmetscherInnen, KollegInnen oder persönliche Kontakte können bei der Informationsakquise eine bedeutende Rolle spielen. An Relevanz als Informationsquelle nimmt auch das Internet zu. Daraus ergibt sich die wesentliche Frage: Wer definiert nun, was ein Fakt ist? Welche sind die autorisierten Quellen, wer sind die AkteurInnen mit Definitionsmacht darüber, was als Faktum zu betrachten ist?

Wenn EntscheiderInnen Informationen über die aktuelle Situation in einem Land suchen, können sie beispielsweise Anfragen an die Staatendokumentation stellen, die 2005 im ehemaligen Bundesasylamt etabliert wurde. Dieser Einrichtung kommt per Gesetz eine bedeutende Rolle in der Definition von Fakten zu. "Zweck der Staatendokumentation ist insbesondere die Sammlung von Tatsachen, die relevant sind (1) für die Beurteilung, ob Tatsachen vorliegen, die auf die Gefahr von Verfolgung im Sinne des AsylG 2005 in einem bestimmten Staat schließen lassen; (2) für die Beurteilung der Glaubwürdigkeit der Angaben von Asylwerbern und Fremden und (3) für die Entscheidung, ob ein bestimmter Staat 
sicher im Sinne der $\S \S 4$ oder 4a AsylG 2005 oder im Sinne der $\S \S 19$ oder 21 BFA-VG ist. «" (AsylG 2005, eigene Hervorhebung)

Anfragen an die Staatendokumentation können beispielsweise lauten: »Sind die folgenden Medikamente für Asylsuchende in Griechenland verfügbar? « oder »Hat Partei XY einen Sitz in Tiflis? «. Welche Fragen ein/e EntscheiderIn stellt und wie diese formuliert werden, kann allerdings die Antworten beeinflussen, darüber sind sich diese im Klaren. Eine Entscheiderin erzählt beispielsweise: "Ich wollte sie [die Asylsuchende] positiv machen. [...] Ich hab die Anfrage an die Staatendoku so geschrieben, dass ich schon gewusst habe, was sie antworten werden, so dass es dann nicht so gut wäre, wenn sie zurückgehen würde.« (Beobachtungsnotiz 0804) Strategisches Handeln ermöglicht so, das Ergebnis der Anfrage mitzugestalten und dadurch indirekt Definitionsmacht darüber zu erlangen, was letztlich als Fakt gehandelt wird. Denn die Antworten der Staatendokumentation - eine autorisierte Quelle - werden in der Regel als Fakten herangezogen, die die Entscheidung legitimieren.

Die Staatendokumentation wiederum erhält ihre Informationen aus diversen Quellen, unter anderem durch sogenannte Verbindungsbeamte, die im jeweiligen Land vor Ort sind und Informationen >aus erster Hand « produzieren.

»Wir haben das eben über den Verbindungsbeamten dann gespielt, ah, dass der dort hin gefahren ist und die Leute befragt hat, ob gefragt hat: Heast, war da mal eine Schneiderei? Das soll die Heratsstraße, was weiß ich, 27 sein, sag ich jetzt einmal. Na wenn natürlich dann [von der] Staatendoku-Recherche die Antwort kommt und [die] sagt, na bitte, dort ist ein Feld oder dort steht eine Kirche und dort war die [...] letzten 26 Jahre nie ein Schneidergeschäft, ja dann tut's mir furchtbar leid, nicht.« (S 0:19)

Die bedeutende Rolle dieser InformantInnen im Rahmen der `Faktisierung` oder Definition von Fakten wird anhand dieses Zitats einer Entscheiderin ersichtlich. Letztere sieht keinen Grund, weshalb sie den Ausführungen der/s Asylsuchenden Glauben schenken sollte, wenn der Informant eine andere Tatsache feststellt. Die Arbeitsweise der InformantInnen steht jedoch nicht unter der Kontrolle der EntscheiderInnen. Ein Entscheider erwähnt beispielsweise, dass er mit der Arbeit eines derzeitigen Verbindungsbeamten in einem bestimmten Land unzufrieden ist. Er erinnert sich an einen Fall, in dem er froh darüber war, dass der alte Kollege, auf den er zählen konnte, noch vor Ort war: »Den, was wir jetzt haben, naja, bemüht sich auch, aber weiß ich nicht; an den Mayer* [Nachname des vorigen Verbindungsbeamten] kommt er nicht heran.« (R 0:11) Während eine der

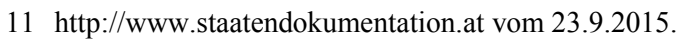


Entscheiderinnen sich dessen bewusst ist, dass »die Staatendoku auch irren [kann] « (S 1:04), scheint es doch in ihrem Arbeitsalltag kaum Alternativen dazu zu geben, die Berichte dieser Verwaltungseinheit zu akzeptieren. Denn die InformantInnen sind qua Amt autorisiert.

Eine weitere legitime Informationsquelle für die Etablierung von Fakten stellen Gutachten dar, wie zum Beispiel medizinische Gutachten (etwa im Kontext von Folter oder Vergewaltigung), psychologische Gutachten (etwa ob eine Person unter Depressionen oder PTSD leidet) oder auch linguistische Gutachten (etwa um die Herkunftsregion einer Person zu bestimmen). In der vorliegenden Untersuchung hat sich allerdings gezeigt, dass im Rahmen einer solchen Beauftragung nicht nur das Artefakt, das heißt das Resultat als verschriftlichte Expertise, sondern auch die Auskunft gebende Person ausschlaggebend sein kann. EntscheiderInnen verfügen über Handlungsspielraum in der Auswahl und Bestellung von GutachterInnen was dazu beiträgt, dass die Beziehung zwischen EntscheiderIn und GutachterIn durchaus von Bedeutung sein kann. Eine Entscheiderin erklärt zum Beispiel, dass sie für ein medizinisches Gutachten keinen unbekannten Gutachter wählen würde, denn »es muss ja ein Arzt des Vertrauens auch sein.« (S 0:03) Sie erzählt, dass sie einen bestimmten Arzt gefragt hat, ob er Gutachten für die Behörde machen würde und drückt nun, da er dies tut, ihre Zufriedenheit mit seiner Arbeit bzw. ihrer Zusammenarbeit aus: »Ich muss sagen, mit dem fahr'ma ganz gut.« (Ebd.) Diese Aussage weist auf die komplexen Machtbeziehungen zwischen ExpertInnen und Behörde hin.

Folgt man Möllering, so beruht Vertrauen darauf, dass Ungewissheit ausgeschlossen werden kann. Außerdem besteht ein Zusammenhang von Vertrauen und Kontrolle, da das eine das andere voraussetzt und gleichzeitig hervorbringt (vgl. Möllering 2005: 283). Vor diesem Hintergrund gewinnt die scheinbar simple Beziehung von AuftraggeberIn und AuftragnehmerIn, zwischen ReferentIn und GutachterIn, an Komplexität. Ausschlaggebend ist in jedem Fall, dass die von einer/einem GutachterIn gemachten Aussagen als unverfälschte Darstellungen der Realität betrachtet werden können, damit ein Gutachten als akzeptabel gelten kann (vgl. ebd.: 65). Im Asylverfahren können, wie auch in anderen Rechtsverfahren, in der Regel alternative Versionen der Realität ins Spiel kommen, die von der/dem EntscheiderIn gegeneinander abgewogen werden müssen.

Anhand der geschilderten Beispiele wird ersichtlich, welche AkteurInnen im Rahmen der Faktensuche und -bestimmung über Einfluss und somit über Definitionsmacht verfügen. Die Staatendokumentation und die mit ihr verbundenen AkteurInnen, wie etwa VerbindungsbeamtInnen, einerseits und GutachterInnen andererseits spielen in diesem Konstruktionsprozess eine bedeutende Rolle. Das Ziel der Behörde, durch ExpertInnen- und institutionalisiertes Wissen Objektivi- 
tät herzustellen, setzt voraus, dass eine klare Unterscheidung zwischen wahr und falsch möglich ist: »[t]he interpretation of the refugee's story in terms of conformity and deviance relies on expert (institutional) knowledge and on an expert experience that must appear to be founded on an objectification of truth and falsehood and that therefore assumes, from the outset, that such objectification is possible.«(Rousseau/Foxen 2006: 515)

Die `Faktisierung`, die soziale Konstruktion von Fakten, und die damit verknüpfte Konstruktion von Un-/Glaubwürdigkeit ${ }^{12}$ sind soziale Praktiken, die dem Dilemma zwischen Eindeutigkeit, respektive Bestimmtheit, einerseits und Ungewissheit andererseits entspringen. Sie repräsentieren ein Beispiel der ständig stattfindenden Transformation von Informalität in Formalität, das heißt auch von Objektvierungsprozessen in der Asylantragsbearbeitung. Als Rechtsverfahren verlangt das Asylverfahren eine eindeutige Entscheidung, gleichzeitig ist der Arbeitsalltag der EntscheiderInnen von permanenter Ungewissheit geprägt. Wie lassen sich diese gegensätzlichen Pole vereinbaren? Um Beweise schaffen zu können, müssen zunächst Fakten geschaffen werden. Informationen werden durch Länderberichte, Gutachten etc. eingeholt, um dem Problem der Unentscheidbarkeit $\mathrm{zu}$ begegnen und schlussendlich $\mathrm{zu}$ einer Entscheidung zu kommen. ${ }^{13}$ Mangels harter Beweise für die von einer/m Asylsuchenden vorgebrachte soziale Realität werden im Zuge dieser Entscheidungsfindung auch Fakten konstruiert, indem Möglichkeiten und (ExpertInnen-)Meinungen den Stellenwert von Tatsachen erlangen. Denn der Bescheid muss sich auf Fakten stützen und in der Argumentation enthaltene Schlussfolgerungen müssen als logisch erscheinen, auch um einer eventuellen Beschwerde standzuhalten. Mit der entsprechenden Argumentation wird schließlich der abstrakte Rahmen der Formalität gefüllt und die Entscheidung somit »extern über eine verbindliche Verfahrensregelung« legitimiert (Froschauer/Lueger 2015: 205).

\subsection{Formalität durch Schriftlichkeit}

Die Bedeutung der Schriftlichkeit in Rechtsverfahren ist massiv: »Quod non est in actis, non est in mundo ${ }^{14}$. Dieser mit dem römischen Recht und der Schriftlichkeit gerichtlicher Verfahren eingeführte Grundsatz illustriert, dass all das,

12 Das Konzept der Un-/Glaubwürdigkeit stellt ein wesentliches Element der Entscheidung über Asylanträge dar (vgl. Sweeney 2009).

13 Die Entscheidung ist allerdings insofern nicht definitiv, als gegen sie beim Bundesverwaltungsgericht (früher beim Asylgerichtshof) Beschwerde eingelegt werden kann.

$14 »$ Was nicht in den Akten ist, ist nicht in der Welt«. 
was nicht - schriftlich - in den Akten festgehalten ist, für das Verfahren nicht existiert. Was nicht vorgetragen ist und somit nicht in die Akte gelangt, wird bei der richterlichen Entscheidung nicht berücksichtigt. ${ }^{15}$ Rechtliche Verfahren basieren in der modernen Verwaltung auf dem Prinzip der Schriftlichkeit und Aktenförmigkeit; dies schreibt bereits Weber (1972[1922]). Baecker (1994) bezeichnet das Gebot der Schriftlichkeit bzw. Aktenförmigkeit als das »Herzstück« der Bürokratie. »Ein trickreicheres Instrument zur Abschottung von eben dadurch erst ermöglichten Entscheidungsvorgängen ist selten erfunden worden. Entscheidung folgt auf Entscheidung, wenn und nur wenn sie als Aktenvorgang darstellbar und fixierbar ist « (Baecker 1994: 23). Ein wichtiger Punkt ist jedoch, dass nur das dokumentiert wird, was von den Verantwortlichen als relevant erachtet wird. Insofern ist augenscheinlich, dass auch im Asylverfahren durch diesen Selektionsprozess einiges >auf der Strecke< bleibt und höchstens zwischen den Zeilen zu finden ist und somit informell bleibt (vgl. Dahlvik 2013).

Dies gilt nicht nur für die Aktenerstellung im Allgemeinen, sondern wird (nicht nur) im Asylverfahren gerade bei der Protokollierung von Einvernahmen besonders relevant. Denn der Prozess der Protokollerstellung lässt sich auch als >gefilterte< Kommunikation fassen (vgl. Dahlvik 2010). Das offizielle, formale Protokoll stellt eine Rekonstruktion dessen dar, was die/der Asylsuchende ursprünglich gesagt hat, nachdem es die/den DolmetscherIn und den »Wie soll ich es formulieren«-Filter des/der EntscheiderIn passiert hat. »The flight motives of an applicant are always product of choices made by people involved in getting them down on paper - the applicant, the translator, the interview official, the lawyer, the decision maker.« (Spijkerboer 2000: 46) Blommaert (2001: 415) spricht von der (Re-)Strukturierung von Gesprochenem in institutionell sanktionierten Text.

Insgesamt macht Schreiben einen wesentlichen Teil der Arbeit der EntscheiderInnen aus. Wenn es darum geht, die Entscheidung über einen Asylantrag zu argumentieren, unterscheidet einer der EntscheiderInnen zwischen zwei Elementen: einerseits zählen die Fakten des Falls, andererseits kommen auch die Ein-

15 Während Schriftlichkeit einerseits die Reichweite der Kommunikation erhöht, indem sie diese von Zeit und Ort der Mitteilung unabhängig macht, gefährdet sie zugleich deren Gelingen, da situative Verständigungshinweise und unmittelbare Klärungsmöglichkeiten entfallen (vgl. Nüsken 2008). Wie Wolff (2008: 502) anmerkt, kann »das für Organisationen typische Beharren auf schriftlichen Dokumenten als der präferierten Form der Darstellung von Wirklichkeit zu schmerzlichen Differenzerfahrungen führen, insbesondere dann, wenn Personen mit den amtlichen Darstellungen von Abläufen konfrontiert werden, an denen sie selbst beteiligt waren«. 
drücke ins Spiel, die der Entscheider im Rahmen der direkten Interaktion mit der/m Asylsuchenden gewonnen hat (W 0:06). Diese Eindrücke betreffen zum Beispiel das Verhalten oder das Auftreten der Person in der Einvernahme. Es bleibt allerdings unklar, ob der Entscheider den inhaltlichen Fakten oder seinen subjektiven Einschätzungen Priorität einräumt, denn er nennt keine Reihenfolge, sondern meint, man müsse beides gleichzeitig berücksichtigen. In der formalen schriftlichen Entscheidung ist jedoch kaum Platz für informelle, subjektive Eindrücke und darauf basierende Schlussfolgerungen bezüglich der >Wahrheit< oder Wahrscheinlichkeit des Vorbringens. Jedenfalls müssen letztere durch einen Transformationsprozess in ein formales Format gebracht werden. Es kann demnach auch die Spannung zwischen Objektivität und Subjektivität im Asylverfahren - im Rahmen der Faktensuche und Glaubwürdigkeitsprüfung - im Licht der Formalität und Informalität betrachtet werden. Gerade in der Suche nach Fakten, die im vorigen Abschnitt behandelt wurde, zeigt sich neuerlich die Bedeutung der Schriftlichkeit im Asylverfahren. Denn schriftliche Quellen haben meist mehr Gewicht als mündliche Aussagen - was niedergeschrieben ist, hat Gültigkeit. »[C]ertain documents and the interpretation they afford permit the determination of the organizationally relevant $>$ facts $<$ of the case independently of the applicant's statement «. (Zimmerman 1976: 338) Zu diesen vertrauenswürdigen Quellen zählen beispielsweise auch Gutachten, die von EntscheiderInnen in Auftrag gegeben werden, um als Unterstützung für die Entscheidungsfindung in konkreten Fällen zu funktionieren.

\section{Conclusio und Ausblick}

Die hier vorgestellte organisationssoziologische Perspektive wirft neues Licht auf staatliches Handeln in der Asylverwaltung. Die Kombination aus Strukturations- und Praxistheorie mit Blick auf soziales Handeln in Organisationen hilft, das Handeln der Akteure, eingebettet in strukturelle Zusammenhänge, zu erklären. Anhand der Analyse konnte erstens aufgezeigt werden, dass das Entscheiden über Asylanträge auf administrativer Ebene zum Teil auf der sozialen Konstruktion von Fakten basiert. Zweitens wurde deutlich, dass diese Konstruktionen durch strukturelle Spannungen im Asylverfahren (aufgrund von Rahmenbedingungen wie Gesetze, Bürokratie, Politik etc.) notwendig erscheinen. Drittens hat es sich als sinnvoll erwiesen, die durch diese Spannungen hervorgebrachten sozialen Praktiken (wie die der Konstruktion) als Zusammenspiel von Formalität und Informalität in Organisationen zu verstehen. 
Das soziologische Konzept der Funktionalität des Zusammenspiels von Formalität und Informalität in Organisationen macht die beobachteten sozialen Praxen - konkret den Umgang der EntscheiderInnen mit den identifizierten Dilemmata - verständlich. Die Betrachtung der vier Spannungsfelder - Eindeutigkeit vs. Ungewissheit; Normierung vs. Handlungsspielraum; Individualisierung vs. Generalisierung; Verantwortung vs. Distanzierung - unter dem Gesichtspunkt der organisationalen Formalität und Informalität macht sichtbar, wie diese zwei Bereiche im administrativen Asylverfahren koexistieren und unterschiedliche Bereiche regeln. So bilden Normierung, Individualisierung, Entscheidung und Verantwortung den formalen Rahmen, während Handlungsspielraum, Ungewissheit, Generalisierung und Distanzierung die Praxis des Arbeitsalltags in der Behörde auf informeller Ebene definieren.

Die Verknüpfung der empirischen Ergebnisse mit den gewählten theoretischen Zugängen zeigt, dass die geschilderten sozialen Praktiken und Strukturen im Asylverfahren einander - im Sinne Giddens' (2011[1986]) Dualität der Struktur - wechselseitig beeinflussen. Im Zentrum der Analyse stand die Beziehung zwischen praktischem Wissen, sozialem Handeln und strukturellen Dilemmata im administrativen Asylverfahren auf theoretischer sowie auf praktischer Ebene. Durch den ethnographischen Zugang wurde deutlich, dass die sozialen Praxen der EntscheiderInnen im Arbeitsalltag häufig auf praktischem Wissen und einer informellen Logik basieren. EntscheiderInnen erlangen ihre professionelle >Reife〈 durch »learning by doing«, sie bilden informelle Kategorien für Asylsuchende und ihre >Fälle`, und pflegen oftmals individuelle Beziehungen zu den GutachterInnen. Die rechtliche und institutionelle Struktur, in der die Bearbeitung von Asylanträgen stattfindet, erfordert jedoch Formalität, das heißt nicht nur einen stabilen Rahmen für soziale Prozesse, sondern auch Legitimation durch verbindliche Verfahrensregelung (vgl. Froschauer/Lueger 2015: 205). Um eine Entscheidung offiziell und formell brauchbar $\mathrm{zu}$ machen, muss sie also in den Rahmen der Formalität eingepasst werden. Die verschiedenen Prozesse der Transformation auf eine formale Ebene - zum Beispiel durch die oben beschriebene soziale Konstruktion von Fakten - muss nun im Rahmen sozialer Praxen stattfinden und ist dort auf der Ebene des >doing`von Asylentscheidungen beobachtbar. Soziales Handeln ist also Handeln in Hinblick auf die strukturellen Dilemmata, wobei Handlungsspielräume ein >Dehnen` der Struktur in die eine oder andere Richtung ermöglichen. Gleichzeitig bilden die vorherrschenden Strukturen einen Bezugsrahmen für das Handeln der AkteurInnen und beeinflussen so deren Möglichkeiten.

Die beschriebenen, durchaus komplexen Prozesse der Formalisierung dienen - paradoxerweise - schlussendlich der Komplexitätsreduktion: Die Realität (Er- 
fahrungen der Asylsuchenden) muss auf die rechtlichen und administrativen Anforderungen zugeschnitten werden. Komplexitätsreduktion muss auch deshalb stattfinden, da wir prinzipiell nicht genug wissen, »unsere Informationsverarbeitungskapazität begrenzt [ist], und unsere Präferenzen instabil und inkonsistent [sind].« (Ortmann 2003: 138) Hinzu kommen Systemzwänge, Handlungsketten und Pfadabhängigkeiten, die vernünftige Entscheidungen ohnehin unwahrscheinlich machen (vgl. ebd.). Komplexität zu reduzieren ist überdies notwendig, da die Bemühung um größere Genauigkeit - das Immer-Weitersuchen nach >wahren< Fakten - sonst kein Ende finden kann, wodurch die/der AkteurIn letztendlich handlungsunfähig wird (vgl. Reichertz 1997). Schließlich ist das Asylverfahren ein bürokratischer Prozess, in dem Eindeutigkeit produziert werden muss und daher Alternativen ausgeschlossen und verunmöglicht werden. Dies führt dazu, dass »Zweifel unterdrückt, aus schwachen Begründungen starke, aus fehlenden fingierte werden « (Ortmann 1990: 375). Ortmann beschreibt Entscheiden daher als Transformation von Kontingenz und Ambiguität in Eindeutigkeit.

Auf politischer, aber auch institutioneller und organisationaler Ebene macht sich angesichts der geschilderten, das Asylverfahren charakterisierenden Dilemmata die Frage breit, wohin dieses Gefangensein in den Dilemmata führen kann (vgl. Dahlvik/Reinprecht 2015). Ist diese Struktur zukunftsfähig, vielmehr: ist sie erhaltenswert? In Anbetracht der derzeitigen - menschenunwürdigen - Situation vieler Asylsuchender in Europa fragt sich, ob es nicht längst an der Zeit ist, die festgefahrenen Strukturen aufzubrechen und neue anzudenken, anstatt ein System, das angesichts der nicht aufzulösenden strukturellen Dilemmata an sich krankt und sich offenbar in einer Sackgasse befindet, permanent zu reformieren. ${ }^{16}$ Stattdessen sollte über Alternativen und Wege nachgedacht werden, die zeitgemäße Formen der Migration ermöglichen und über die EUBinnenmobilität hinausgehen.

16 Ich denke an gesetzliche Änderungen auf nationaler und supranationaler Ebene, beispielsweise die Einführung von Dublin I, II und II, aber auch an die zahlreichen institutionellen Veränderungen in Österreich in den letzten Jahren (vom Unabhängigen Bundesasylsenat zum Asylgerichtshof und dessen letztliche Abschaffung bzw. Integration in das neue Bundesverwaltungsgericht, oder die Verwandlung des Bundesasylamtes in das Bundesamt für Fremdenwesen und Asyl). 


\section{Literatur}

Ammer, Margit/Busch, Brigitta/Dorn, Nora/Rienzner, Martina/Santner-Wolfartsberger, Anita/Schicho, Walter/Seidlhofer, Barbara/Spitzl, Karlheinz (2013): »Ein umstrittenes Beweismittel. Sprachanalyse als Instrument der Herkunftsbestimmung in Asylverfahren«, in: Juridikum 3, S. 281-297.

Arnim-Ellissen, Jakob (2012): Asylmissbrauch - Kampfbegriff der österreichischen Asyldebatte? Unveröffentlichte Diplomarbeit, Universität Wien.

Auzinger, Julia M. (2012): Kultur und Kommunikation im österreichischen Asylverfahren. Unveröffentlichte Masterthesis, Seewalchen am Attersee.

Baecker, Dirk (1994): »Durch diesen schönen Fehler mit sich selbst bekannt gemacht: Das Experiment der Organisation«, in: Lettre International 24, S. 22-26

Behrend, Olaf (2013): »Emotional practices and semantics of public employment agents«, in: Österreichische Zeitschrift für Soziologie 38(2), S. 149-165.

Biegelbauer, Peter (2013): Wie lernt die Politik? Lernen aus Erfahrung in Politik und Verwaltung, Wiesbaden: Springer VS.

Bitoulas, Alexandros (2015): »Asylum applicants and first instance decisions on asylum applications: 2014«, in: Population and social conditions, Eurostat Data in Focus 3/2015, http://ec.europa.eu/eurostat/documents/4168041/6742 650/KS-QA-15-003-EN-N.pdf/b7786ec9-1ad6-4720-8a1d-430fcfc55018 vom 20.05.2016.

Blau, Peter Michael (1955): The Dynamics of Bureaucracy: A Study of Interpersonal Relations in Two Government Agencies, Chicago, IL: University of Chicago Press.

Blommaert, Jan (2001): »Investigating Narrative Inequality: African Asylum Seekers' Stories in Belgium«, in: Discourse and Society 12(413), S. 413-449.

Böhle, Fritz, G./Voß, Günter/ Wachtler, Günther (2010): Handbuch Arbeitssoziologie, Wiesbaden: VS Verlag für Sozialwissenschaften.

Böhle, Fritz/Bolte, Annegret (2002): Die Entdeckung des Informellen. Der schwierige Umgang mit Kooperation im Arbeitsalltag, Frankfurt a.M./New York: Campus.

Bolte, Annegret/Porschen, Stephanie (2006): Die Organisation des Informellen, Wiesbaden: VS Verlag für Sozialwissenschaften.

Bourdieu, Pierre (1972): Esquisse d'une théorie de la pratique, Genf: Droz.

Bundesgesetz über die Gewährung von Asyl (Asylgesetz 2005 - AsylG 2005), https://www.ris.bka.gv.at/GeltendeFassung.wxe?Abfrage=Bundesnormen \& Gesetzesnummer $=20004240$ vom 23.9.2015. 
Dahlvik, Julia (2010): »Asylanhörungen: Handlungsspielräume in DolmetschInteraktionen «, in: Stichproben - Wiener Zeitschrift Für Kritische Afrikastudien 10(19), S. 63-82.

Dahlvik, Julia (2013): »Institutionelle Einsichten: Die Bedeutsamkeit von Schriftlichkeit und Dokumenten im Prozess der Bearbeitung von Asylanträgen«, in: Julia Dahlvik/Christoph Reinprecht/Wiebke Sievers (Hg.), Migration und Integration - wissenschaftliche Perspektiven aus Österreich: Jahrbuch 2/2013, Göttingen: V\&R unipress, S. 301-317.

Dahlvik, Julia (2014): Administering Asylum Applications. Unveröffentlichte Dissertation, Universität Wien.

Dahlvik, Julia/Reinprecht, Christoph (2015): »Asyl als Widerspruch - vom Menschenrecht zum Auserwählten?«, in: Pia Janke (Hg.), JELINEK[JAHR] BUCH. Elfriede Jelinek-Forschungszentrum 2014-2015, Wien: Praesens Verlag, S. 43-54.

Friedberg, Erhard (1995): Ordnung und Macht. Dynamiken organisierten Handelns, Frankfurt a. M. u.a.: Campus-Verlag.

Fritsche, Andrea (2012): »Zeit. Macht. Flüchtlinge. Und Flüchtlinge machen Zeit? Konzeptionen biografischer Zeiten im Asylkontext«, in: SWSRundschau 52(4), S. 362-388.

Froschauer, Ulrike/Lueger, Manfred (2009): Interpretative Sozialforschung: der Prozess, Wien: Facultas WUV.

Froschauer, Ulrike/Lueger, Manfred (2015): »Informalität als organisationaler Basisrhythmus«, in: Victoria von Groddeck/Sylvia Marlene Wilz (Hg.), Formalität und Informalität in Organisationen (=Organisationssoziologie), Wiesbaden: Springer Fachmedien, S. 191-213.

Geertz, Clifford (1983): Local Knowledge: Further Essays in Interpretive Anthropology, New York, NY: Basic Books.

Giddens, Anthony (2011[1986]): The Constitution of Society: Outline of the Theory of Structuration, Cambridge u.a.: Polity Press.

Grießler, Erich (2003): »Innovation und Politikgestaltung: Administrative Kulturen in der Technologiepolitik - ein Vergleich zwischen Österreich und den Niederlanden«, in: Rupert Pichler (Hg.), Innovationsmuster in der österreichischen Wirtschaftsgeschichte. Wirtschaftliche Entwicklung, Unternehmen, Politik und Innovationsverhalten im 19. und 20. Jahrhundert, Innsbruck u.a.: Studien Verlag, S. 290-312.

Grunow, Dieter (2009): »Bürokratieforschung«, in: Viktoria Kaina/Andrea Römmele (Hg.), Politische Soziologie, Wiesbaden: VS Verlag für Sozialwissenschaften, S. 353-383. 
Hupe, Peter (2013): »Dimensions of Discretion: Specifying the Object of StreetLevel Bureaucracy«, in: dms - der moderne staat - Zeitschrift für Public Policy, Recht und Management 6(2), S. 425-440

Jubany, Olga (2011): »Constructing Truths in a Culture of Disbelief «, in: International Sociology 26(1), S. 74-94.

Knoblauch, Hubert/Heath, Christian (1999): »Technologie, Interaktion und Organisation. Die Workplace-Studies«, in: Schweizerische Zeitschrift für Soziologie 25(2), S. 163-181.

Lautmann, Rüdiger (2011): Justiz - Die Stille Gewalt. Teilnehmende Beobachtung und entscheidungssoziologische Analyse, Wiesbaden: VS Verlag für Sozialwissenschaften.

Lipsky, Michael (2010[1980]): Streel-Level Bureaucracy: Dilemmas of the Individual in Public Services, 30th anniversary expanded version, New York: Russell Sage Foundation.

Maynard-Moody, Steven/Portillo, Shannon (2010): »Street-Level Bureaucracy Theory«, in: Robert F. Durant/George C. Edwards III (Hg.), The Oxford Handbook of American Bureaucracy, Oxford: Oxford University Press, S. 252-277.

Mayntz, Renate (1997): Soziologie der öffentlichen Verwaltung. 4., durchges. Aufl., Heidelberg: Müller.

Mica, Adriana/Winczorek, Jan/Wisniewski, Rafal (Hg.) (2015): Sociologies of Formality and Informality, Frankfurt a. M./New York: Peter Lang.

Möllering, Guido (2005): »The Trust/Control Duality: An Integrative Perspective on Positive Expectations of Others«, in: International Sociology 20(3), S. 283-305.

Nüsken, Dirk (2008): Regionale Disparitäten in der Kinder- und Jugendhilfe. Eine empirische Untersuchung zu den Hilfen für junge Volljährige, Münster/New York: Waxmann.

Ortmann, Günther/Sydow, Jörg/Windeler, Arnold (2000): »Organisation als reflexive Strukturation«, in: Günther Ortmann/Jörg Sydow/Klaus Türk (Hg.), Theorien der Organisation: die Rückkehr der Gesellschaft, Opladen: Westdeutscher Verlag, S. 315-345.

Ortmann, Günther (2003): Regel und Ausnahme. Paradoxien sozialer Ordnung, Frankfurt a. M.: Suhrkamp.

Ortmann, Günther (1990): Computer und Macht in Organisationen: Mikropolitische Analysen, Opladen: Westdeutscher Verlag.

Plutzar, Verena (2009): Zwischen >Angst $\iota$ und `Zeit - Zur Kommunikationssituation und Informationsweitergabe im Asylverfahren. Dissertation, Universität Wien. 
Pöllabauer, Sonja (2005): »I don’t understand your English, Miss «: Dolmetschen bei Asylanhörungen, Tübingen: Gunter Narr Verlag.

Probst, Johanna (2013): »Asylanträge bearbeiten: BAMF und OFPRA im Vergleich«, in: Ministerium für Integration Baden-Württemberg (Hg.), Integrationspolitik im internationalen Vergleich, Stuttgart, http://www.migpolgroup. com/wp_mpg/wp-content/uploads/2013/08/2013-08-12-Integrationspolitik. pdf vom 20.05.201620.5.2016, S. 54-56.

Prottas, Jeffrey M. (1979): People-Processing: The Street-Level Bureaucrat in Public Service Bureaucracies, Toronto: Lexington Books.

Reckwitz, Andreas. (2003): »Grundelemente einer Theorie sozialer Praktiken. Eine sozialtheoretische Perspektive« in: Zeitschrift für Soziologie 32(4), S. 282-301.

Reichertz, Jo (1997): »Plädoyer für das Ende einer Methodologiedebatte bis zur letzten Konsequenz«, in: Tilmann Sutter (Hg.), Beobachtung verstehen Verstehen beobachten. Perspektiven einer konstruktivistischen Hermeneutik, Wiesbaden: VS Verlag für Sozialwissenschaften, S. 98-133.

Rosenberger, Sieglinde (2010): Asylpolitik in Österreich : Unterbringung im Fokus, Wien: Facultas WUV.

Rousseau, Cécile/Foxen, Patricia (2006): »Le Mythe Du Réfugié Menteur: Un Mensonge Indispensable ?«, in: L’Évolution Psychiatrique 71(3), S. 505-520. Scheffer, Thomas (2001): Asylgewährung. Eine ethnographische Analyse des deutschen Asylverfahrens, Stuttgart: Lucius \& Lucius.

Schumacher, Sebastian/Peyrl, Johannes/Neugschwendtner, Thomas (2015): Fremdenrecht Asyl, Ausländerbeschäftigung, Einbürgerung, Einwanderung, Verwaltungsverfahren. 5., aktualisierte Auflage, Wien: ÖGB-Verlag.

Smith, Dorothy E. (2006): Institutional Ethnography as Practice, Lanham, MD.: Rowman \& Littlefield.

Sondermann, Ariadne/Englert, Kathrin (2013): ») Ich versuch hier auch immer so dieses Amtliche irgendwie noch 'n bisschen zu überspielen`. Emotions- und Gefühlsarbeit in der öffentlichen Verwaltung als Ausdruck von Staatlichkeit im Wandel«, in: Österreichische Zeitschrift für Soziologie 38(2), S. 131-147.

Spijkerboer, Thomas (2000): Gender and Refugee Status, Aldershot, UK: Ashgate/Dartmouth.

Spire, Alexis (2007): »L'asile au guichet. La dépolitisation du droit des étrangers par le travail bureaucratique «, in: Actes de la recherche en sciences sociales 169(4), S. 4-21.

Stinchcombe, Arthur L. (2001): When Formality Works: Authority and Abstraction in Law and Organizations, Chicago, IL u.a.: University of Chicago Press. 
Strauss, Anselm L./Corbin, Juliet (1990): Basics of Qualitative Research: Grounded Theory Procedures and Techniques, London: Sage Publications.

Sweeney, James A. (2009): »Credibility, Proof and Refugee Law«, in: International Journal of Refugee Law 21(4), S. 700-726.

Sydow, Jörg/Wirth, Carsten (Hg.) (2014): Organisation und Strukturation: Eine fallbasierte Einführung, Wiesbaden: Springer VS.

Weber, Max (1972[1922]): Wirtschaft und Gesellschaft, Frankfurt a. M.: Uniprint.

Weiler, Joseph H. H. (1992): »Thou Shalt Not Oppress a Stranger: On the Judicial Protection of the Human Rights of Non-EC Nationals - a Critique $\ll$, in: European Journal of International Law 3(65), S. 65-91.

Wolff, Stephan. (2008): »Dokumenten- und Aktenanalyse«, in: Uwe Flick/Ernst von Kardorff/Ines Steinke (Hg.), Qualitative Forschung: Ein Handbuch, Reinbek: Rowohlt, S. 502-514.

Zimmerman, Don H. (1976): »Recordkeeping and the Intake Process in a Public Welfare Agency«, in: Stanton Wheeler (Hg.), On Record: Files and Dossiers in American Life, New Brunswick, NJ: Transaction Books, S. 319-354. 



\title{
Asyl-Verwaltung kraft Wissen \\ Die Herstellung von Entscheidungswissen \\ in einer Schweizer Asylbehörde
}

LAURA AFFOLTER

\section{Einleitung}

\begin{abstract}
»Die bürokratische Verwaltung bedeutet: Herrschaft kraft Wissen: dies ist ihr spezifisch rationaler Grundcharakter. Über die durch das Fachwissen bedingte gewaltige Machtstellung hinaus hat die Bürokratie (oder der Herr, der sich ihrer bedient), die Tendenz die Macht noch weiter zu steigern durch das Dienstwissen: die durch Dienstverkehr erworbenen oder >aktenkundigen Tatsachenkenntnisse.«

(WEBER 2005: 165F.)
\end{abstract}

Wissensgenerierung ist ein zentrales Charakteristikum von bürokratischen Institutionen. Bürokratien produzieren Wissen (Hoag 2011: 81). Sie eignen sich Wissen über ihr Gegenstandsgebiet an und formen dieses gleichzeitig mit (Heyman 2004: 490). Laut Weber ist das Wissen, auf dem die Macht von Bürokratien beruht, ein ganz spezifisches. Es ist ein »Wissen von zweierlei Art«: Fachwissen und Dienstwissen (1988: 351). Als Fachwissen definiert er das »durch Fachschulung erworbene [...] im weitesten Sinne des Wortes stechnische [...]« Wissen (ebd.). Dienstwissen stellt für ihn die »nur dem Beamten zugängliche Kenntnis der für sein Verhalten maßgebenden konkreten Tatsachen« dar (ebd.). Während Fachwissen prinzipiell für alle zugänglich ist, handelt es sich bei Dienstwissen 
meist um Wissen, das »nicht generell verfügbar« ist (Derlien/Böhme/Heindl 2011: 92).

Will man das Funktionieren von Bürokratien verstehen, verlangt dies nach einer Auseinandersetzung damit, wie sie Wissen produzieren und mobilisieren. In meinem Beitrag zeige ich dies am Beispiel von decision-making im Schweizerischen Asylverfahren durch das Staatssekretariat für Migration (SEM) auf. ${ }^{1}$ Aufbauend auf Webers Definition von »bürokratischem Wissen« argumentiere ich erstens, dass im Entscheidungsprozess ein ganz bestimmtes Wissen generiert wird, das ich pragmatisch »Entscheidungswissen« nenne. Spezifisch ist dem Entscheidungswissen, dass es immer ein klares Endprodukt zum Ziel hat und auf dieses hinführt. Dieses Endprodukt ist der fertige Asylentscheid. Zweitens argumentiere ich, dass im Entscheidungsprozess eine weitere Form von Wissen eine wichtige Rolle spielt. Ich nenne diese Form »professionelles Wissen«. Professionelles Wissen bezeichnet jenes Wissen, das zur Ausführung einer Arbeit - im SEM wesentlich das Fällen von Asylentscheiden - nötig ist, jedoch in keiner schriftlichen Form vorliegt. Ich verwende den Begriff sowohl dafür, was die Entscheider_innen im SEM aus Erfahrung wissen, als auch dafür, was die Entscheider_innen selbst als >Bauchgefühlく bezeichnen. ${ }^{2}$ Er steht für das >einfach wissen ‘, ohne dass man genau sagen könnte, warum man etwas weiß. Professionelles Wissen bildet einen Teil des institutionellen Habitus von Sachbearbeitenden, da es ein Wahrnehmungs-, Denk- und Handlungsschema darstellt, das sich die Sachbearbeitenden durch ihre Arbeit in der Institution angeeignet haben (vgl. Steiner 2001: 24, 41f.; Bourdieu 1976). Da Sachbearbeitende sich professionelles Wissen (unbewusst) durch die institutionelle Sozialisierung aneignen müssen, bedeutet dies, dass diese Form von Wissen nicht generell zugänglich ist. Ich verstehe professionelles Wissen deshalb als eine weitere Form von Dienstwissen im Sinne Webers. Steiner folgend argumentiere ich, dass professionelles Wissen (als Teil des institutionellen Habitus) als »Generierungsprinzip von Verhaltensstrategien « zu verstehen ist. Es »regelt die Praxis, ohne jedoch Regeln zu erfüllen [und es] ist dem Zweck angepasst, ohne Ziele anzuvisieren« (Steiner 2001: $25)^{3}$

1 Das SEM besteht aus mehreren Direktionsbereichen. Ich beschäftige mich in meiner Arbeit ausschließlich mit dem Direktionsbereich Asyl.

2 Die Rolle des >Bauchgefühls` für Asylentscheide wurde unter anderen von Fassin (2013), Jubany (2011), Kelly (2012), Macklin (1998) und Thomas (2009) thematisiert.

3 Mit dem institutionellen Habitus von Sachbearbeitenden setze ich mich in meiner Dissertation genauer auseinander. Ich argumentiere dort, dass es sich bei professionellem Wissen zwar um subjektives Wissen, jedoch nicht um individuelles Wissen handelt. 
In meinem Beitrag gehe ich der Frage nach, wie im Asylverfahren Entscheidungswissen generiert wird. Ich argumentiere dabei, dass Entscheidungswissen das Produkt des Zusammenwirkens von Fachwissen in Form von Länder- und Gesetzeskenntnissen und Dienstwissen in Form von Aktenkundigkeit und professionellem Wissen ist. Dieses Argument leite ich im dritten Teil des Artikels her. Damit das Vorgehen in den Entscheidungsprozessen für die Leser_innen nachvollziehbar wird, zeige ich im zweiten Teil des Artikels die rechtlichen und institutionellen Bedingungen für decision-making im SEM auf.

Der Beitrag basiert auf den Analysen des Datenmaterials aus meiner laufenden Dissertationsforschung, worin ich mich mit der praktischen Entscheidungstätigkeit und im Speziellen mit der Praxis von Glaubhaftigkeitsbeurteilungen im SEM auseinandersetze. Zwischen 2013 und 2015 habe ich in verschiedenen Zentren und Sektionen des SEM ethnographische Forschung durchgeführt. Während meiner Aufenthalte in diesen verschiedenen Teams habe ich die Sachbearbeitenden bei ihrer täglichen Arbeit begleitet; ich habe Asylanhörungen beigewohnt, den Sachbearbeitenden beim Verfassen der Entscheide über die Schulter geschaut, mit ihnen >Fälle ২ diskutiert, an Sektionssitzungen teilgenommen und ich war auch in den Kaffeepausen dabei. Während dreier Wochen konnte ich außerdem an einer internen Ausbildung für neue Mitarbeitende teilnehmen. Meine Beobachtungen und Notizen zu informellen Gesprächen habe ich in Forschungstagebüchern festgehalten. Die Anhörungsprotokolle von >Fällen`, die ich während meiner Aufenthalte in irgendeiner Form begleitet habe, durfte ich kopieren und in anonymisierter Form für mein Forschungsprojekt verwenden, genauso wie die Entscheide zu diesen Gesuchen. Des Weiteren habe ich zu zwei zufällig gesetzten Daten alle Asyldossiers erhalten, über die an den betreffenden Tagen entschieden wurde und durfte daraus Entscheide, Anhörungsprotokolle und andere Dokumente kopieren. Schließlich habe ich semi-strukturierte Interviews mit 25 Sachbearbeitenden geführt, die ich aufgenommen und transkribiert habe. Die Analyse dieses Datenmaterials basiert auf dem Grounded-Theory-Ansatz nach Glaser und Strauss (1967). Ich habe mein Datenmaterial zuerst offen codiert, daraus analytische Kategorien geschaffen und die Beziehungen zwischen diesen Kategorien untersucht (vgl. Bryman 2011: 387ff.). Des Weiteren habe ich sowohl während der Forschung als auch während des Auswertungsprozesses analytische Memos geschrieben (vgl. Hammersley/Atkinson 2007: 150ff.).

Die Kollektivität von professionellem Wissen versuche ich in meiner Dissertation empirisch aufzuzeigen. 


\section{Asylverwaltung im SEM - Der Entscheidungskontext}

Wer in der Schweiz Asyl beantragt, kommt zuerst in eines der fünf Empfangsund Verfahrenszentren (EVZ) nahe der Landesgrenzen und beim Flughafen in Zürich. ${ }^{4}$ Dort findet eine erste kurze Befragung, eine sogenannte Befragung zur Person (BzP), statt, in der die Personalien und der Reiseweg erfragt und die Asylsuchenden gebeten werden, ihre Asylgründe kurz zu schildern. Wochen, Monate oder Jahre danach folgt entweder in einem der EVZ oder in der SEMZentrale in Bern die eigentliche Anhörung zu den Asylgründen. In einzelnen Fällen wird noch eine ergänzende Anhörung durchgeführt. Oft ist es die befragende Person dieser zweiten Anhörung, die den Asylentscheid fällt und ihn zum Kontrollieren und Visieren der Sektionschefin oder dem Sektionschef übergibt. ${ }^{5}$ Der Verwaltungsprozess beginnt also mit dem Erfassen der Personalien der gesuchstellenden Person beim Eintreffen in ein EVZ und endet - zumindest erstinstanzlich - damit, dass die sektionsleitende Person den Entscheid mit einer Unterschrift bestätigt und dieser der gesuchstellenden Person bzw. ihrer Rechtsvertretung zugestellt wird. Dazwischen geschehen jedoch noch einige Schritte.

\subsection{Die Arbeit im SEM}

$\mathrm{Zu}$ den Aufgaben der Sachbearbeitenden in den EVZ gehört es, BzP zu führen und $\mathrm{zu}$ protokollieren, Asylsuchende $\mathrm{zu}$ ihren Asylgründen anzuhören ${ }^{6}$ und Asylentscheide zu schreiben. Die Sachbearbeitenden in der Zentrale führen kei-

4 Ich beziehe mich im Folgenden auf das Verfahren, wie es im Moment noch in den fünf Empfangs- und Verfahrenszentren und in der Zentrale in Bern zur Anwendung kommt. Seit Januar 2014 gibt es in Zürich einen Testbetrieb, in welchem ein beschleunigtes Verfahren durchgeführt wird (https://www.bfm.admin.ch/bfm/de/home/ aktuell/news/2015/2015-02-16.html vom 08.04.15).

5 Andere Anhörungen werden von sogenannten >Poolies« durchgeführt. Die Bezeichnung >Poolies $<$ leitet sich davon ab, dass diese Personen zu einem Pool von Befragenden gehören, die im Stundenlohn arbeiten und keine regelmäßigen Arbeitszeiten haben. >Poolies` führen Anhörungen durch, treffen aber selbst keine Entscheide. Nach Anhörungen durch >Poolies` gehen die Asyldossiers mit dem Wortprotokoll der Anhörung an Sachbearbeitende des SEM, welche die Entscheide fällen.

6 Diese protokollieren sie nicht selbst, denn hier ist neben dem/der Gesuchsteller_in, dem/der Dolmetscher_in und der Hilfswerksvertretung auch ein/e Protokollführer_in anwesend. 
ne BzP durch, dafür haben die meisten von ihnen eine Federführung inne. Federführungen gibt es sowohl für die einzelnen Herkunftsländer als auch für bestimmte Themen, wie zum Beispiel geschlechtsspezifische Verfolgung oder Familienzusammenführung. Die Federführungen sind zuständig für die unité de doctrine (Einheitlichkeit der Entscheidungspraxis), also dafür, dass Gesuche von Asylsuchenden aus gleichen Ländern bzw. mit einem ähnlichen sogenannten Risikoprofil gleich behandelt werden. Das bedeutet nicht, dass die federführenden Personen alle diese Fälle selber behandeln. Sie erstellen und aktualisieren jedoch auf der Basis von wegweisenden (Grundsatz-)Urteilen des Bundesverwaltungsgerichts (BVGer), von Länderinformationen, die sie von den Länderreferentinnen und Länderreferenten ${ }^{7}$ erhalten, sowie von Praxisbestimmungen der Amtsleitung die jeweilige »Asyl- und Wegweisungspraxis«, kurz APPA genannt. APPAs sind eine Art amtsinterner Richtlinien, welche die Asyl- und Wegweisungspraxis in Bezug auf ein bestimmtes Land bzw. auf eine bestimmte Gruppe von Personen aus dem jeweiligen Herkunftsland definieren. ${ }^{8}$ Die federführenden Personen fungieren des Weiteren als Ansprechpersonen für die anderen Sachbearbeitenden bei Fragen, welche die Entscheidungspraxis betreffen.

\subsection{Die rechtlichen Grundlagen}

Das Schweizerische Asylgesetz (AsylG) und das Bundesgesetz über die Ausländer und Ausländerinnen $(\mathrm{AuG})$ bilden die Basis, auf der die institutionelle Entscheidungspraxis generiert und Asylentscheide getroffen werden. Im Entscheidungsprozess müssen die Sachbearbeitenden eine Reihe von Fragen beantworten, die ich in der untenstehenden Grafik vereinfacht dargestellt habe.

7 Die Länderreferentinnen und Länderreferenten arbeiten in der Abteilung »Analysen und Services«. Ihre Aufgabe besteht darin, Einzelfallanfragen von Sachbearbeitenden zu beantworten, Berichte zu schreiben und ein Monitoring in den Ländern durchzuführen, für die sie zuständig sind. Sie sind verantwortlich für die internen Länderinformationen und Datenbanken und bieten ab und zu Ausbildungen zu einzelnen Ländern an. Sie treffen jedoch keine Asylentscheide und bestimmen die Asyl- und Wegweisungspraxis nicht.

8 Die APPAs sind immer gleich aufgebaut. Im ersten Teil wird die allgemeine Situation im Land erklärt, dann folgt ein Teil mit Anweisungen, was in der BzP beachtet bzw. geprüft werden soll und danach ein Teil mit Anweisungen zu den Anhörungen. Nach diesen Anweisungen sind mögliche Asylmotive aufgeführt und die daraus folgenden Entscheide. Das Gleiche gibt es auch in Bezug auf die (Un-)Zumutbarkeit des Wegweisungsvollzugs. Hierbei handelt es sich um sogenannte Risikoprofile. 
Abbildung 1: Schematische Darstellung des Entscheidungsverfahrens

$$
\underset{\text { Nein }}{\rightarrow}
$$

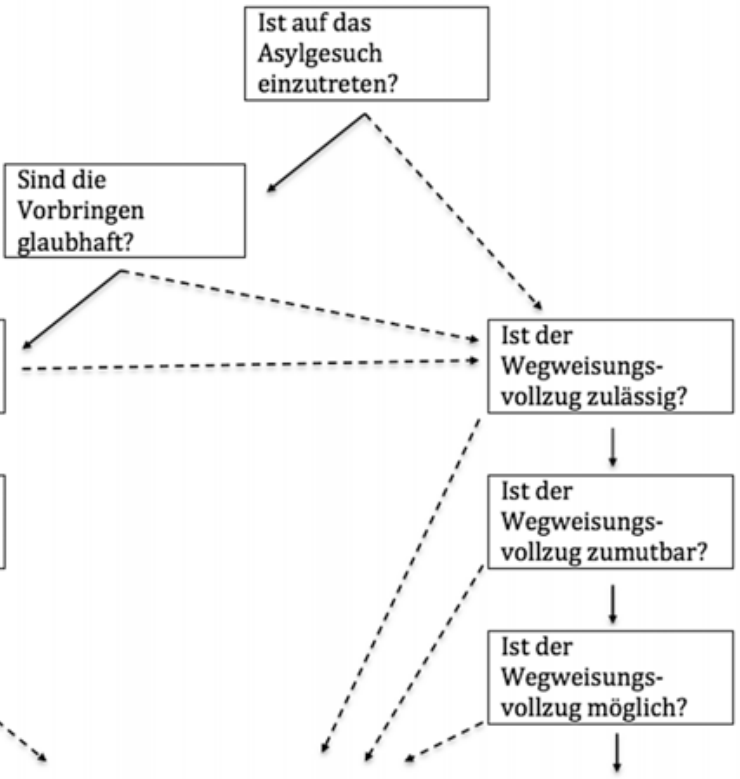

Asylgewährung

Vorläufige
Aufnahme als
Flüchtling

Quelle: Eigene Darstellung auf der Grundlage des AsylG und AuG.

Ausschlaggebend dafür, ob eine Person Asyl erhält oder nicht, sind vor allem die Fragen nach der Flüchtlingseigenschaft gemäß Artikel 3 AsylG und nach der Glaubhaftigkeit gemäß Artikel 7 AsylG. Als Flüchtlinge gelten in der Schweiz Personen, »die in ihrem Heimatstaat oder im Land, in dem sie zuletzt wohnten, wegen ihrer Rasse, Religion, Nationalität, Zugehörigkeit zu einer bestimmten sozialen Gruppe oder wegen ihrer politischen Anschauungen ernsthaften Nachteilen ausgesetzt sind oder begründete Furcht haben, solchen Nachteilen ausgesetzt zu werden« (Art. 3, Abs. 1, AsylG). Dies gilt unter der Voraussetzung, dass keine Asylausschlussgründe (gemäß Art. 1 F Abkommen über die Rechtsstellung der Flüchtlinge und Art. 53 und 54 AsylG) ${ }^{9}$ vorliegen und die Vorbringen ${ }^{10}$

9 Artikel $1 \mathrm{~F}$ der Genfer Flüchtlingskonvention schließt Personen aus, die »ein Verbrechen gegen den Frieden, ein Kriegsverbrechen oder ein Verbrechen gegen die 
der asylsuchenden Person für glaubhaft befunden werden. »Glaubhaft gemacht ist die Flüchtlingseigenschaft, wenn die Behörde ihr Vorhandensein mit überwiegender Wahrscheinlichkeit für gegeben hält.« (Art. 7, Abs. 2, AsylG) Als unglaubhaft gelten die Vorbringen dann, »wenn sie in wesentlichen Punkten zu wenig begründet oder in sich widersprüchlich sind, den Tatsachen nicht entsprechen oder maßgeblich auf gefälschte oder verfälschte Beweismittel abgestützt werden« (Art. 7, Abs. 3, AsylG). Wir erkennen hier, dass sowohl die Flüchtlingseigenschaft als auch die Glaubhaftigkeit mit Wahrscheinlichkeiten begründet werden. So müssen die Sachbearbeitenden sowohl einschätzen, ob sie vorgebrachte vergangene Verfolgungen für überwiegend wahrscheinlich halten, als auch beurteilen, wie groß die Wahrscheinlichkeit zukünftiger Verfolgung ist.

Der Wortlaut »überwiegend wahrscheinlich« aus Artikel 7 AsylG wird von vielen Sachbearbeitenden dahingehend interpretiert, dass etwas - mathematisch betrachtet - zu 51 Prozent wahrscheinlich sein muss, um glaubhaft zu sein. Das wird einerseits als schwierig erachtet, weil Asylentscheide, so die Sachbearbeitenden, keine mathematischen Entscheide seien und die Bestimmung, ob etwas überwiegend wahrscheinlich sei, nach einer >subjektiven` Einschätzung verlange und andererseits, weil eine 51 prozentige Wahrscheinlichkeit Raum lasse für 49 Prozent Zweifel - was viele Sachbearbeitende als >ganz schön viel erachten. Die wahrgenommenen Schwierigkeiten hängen unter anderem mit dem sogenannten >niedrigeren Beweisstandard ‘ des Asylverfahrens beispielsweise im Vergleich zum Strafverfahren zusammen. Artikel 7 AsylG verlangt, dass die Flüchtlingseigenschaft nachgewiesen oder zumindest glaubhaft gemacht wird (Art. 7, Abs. 1, AsylG). Da Fluchtgründe oft materiell nicht bewiesen werden können, genügt in der Praxis meist die Glaubhaftmachung. Dies bedeutet einer-

Menschlichkeit im Sinne der internationalen Vertragswerke begangen haben«, die »ein schweres nichtpolitisches Verbrechen außerhalb des Aufnahmelandes begangen haben, bevor sie dort als Flüchtling aufgenommen wurden« oder die »sich Handlungen zu Schulden kommen ließen, die den Zielen und Grundsätzen der Vereinten Nationen zuwiderlaufen« (Art. 1 F Abkommen über die Rechtstellung der Flüchtlinge). Das Schweizerische Asylgesetz gewährt Flüchtlingen kein Asyl, »wenn sie wegen verwerflicher Handlungen dessen unwürdig sind oder wenn sie die innere oder die äußere Sicherheit der Schweiz verletzt haben oder gefährden« (Art. 53 AsylG) sowie »wenn sie erst durch ihre Ausreise aus dem Heimat- oder Herkunftsstaat oder wegen ihres Verhaltens nach der Ausreise Flüchtlinge im Sinne von Artikel 3 wurden« (Art. 54 AslyG).

10 Das Wort »Vorbringen« steht im Asylgesetz für die Aussagen von Asylsuchenden im Verfahren. 
seits, dass die mündlichen Aussagen von Asylsuchenden (bzw. die davon erstellten Wortprotokolle) die Basis bilden, auf der Asylentscheide getroffen werden. Andererseits bedeutet dieser >niedrigere $<$ Beweisstandard, dass praktisch alles als Beweis eingereicht werden kann und es gleichzeitig keine standardisierten Normen für die Interpretation dieser Beweise gibt (vgl. Kelly 2011: 185, 196) ${ }^{11}$

Im Asylverfahren müssen Sachbearbeitende Geschehnisse beurteilen, an denen sie selbst nicht teilgenommen haben und bei denen sie deshalb nie sicher wissen können, was wirklich passiert ist (vgl. Scheffer 2003: 425). Materielle Beweise und sogenannte Tatsachenkenntnisse kommen aus Sicht der Entscheider_innen einem an Sicherheit grenzenden Wissen am nächsten. ${ }^{12}$ Doch materielle Beweise fehlen häufig. Außerdem fehlt es den Sachbearbeitenden an Möglichkeiten und an Zeit, um solche Tatsachenkenntnisse zu generieren. ${ }^{13}$ Die meisten Sachbearbeitenden arbeiten nämlich unter großem Zeitdruck, da sie quantitative Ziele erreichen müssen, ebenso wie die einzelnen Asylabteilungen und Sektionen. ${ }^{14}$ Für die Glaubhaftigkeitsbeurteilung bedeutet dies laut einem Sachbearbeiter: »Im Strafverfahren gibt es Zeugen und jahrelange Prozesse. Und wir

11 Kelly (2011) zeigt auf, dass obwohl der Beweisstandard im Asylverfahren formell als niedrig gilt, er in der Praxis sehr hoch ist. Darauf gehe ich in meiner Dissertation näher ein.

12 Tatsachenkenntnisse basieren meist auf Dokumenten, denen ein Wahrheitswert zugeschrieben wird. So gilt es zum Beispiel als Tatsachenkenntnis, wenn man aufgrund von Medienberichten oder Länderreports weiß, wann ein bestimmtes Ereignis, wie beispielsweise eine Demonstration, stattgefunden hat und daraus ableiten kann, ob das, was die Person erzählt, >wahr ist oder nicht.

13 Gleichzeitig zeigt mein Datenmaterial, dass materielle Beweise in der Entscheidungspraxis sehr wohl eine Rolle spielen. So wurden 19 der 21 positiven Asylentscheide, die ich analysiert habe, zumindest teilweise mit materiellen Beweisen begründet. Ähnliches haben auch Good (2011) und Kobelinsky (2008) für die Verfahren in Großbritannien und Frankreich festgestellt.

14 Im SEM haben alle Mitarbeitenden quantitative Ziele, die sie erreichen sollten (genauso wie die einzelnen Asylabteilungen und Sektionen). Die Zahlen variieren je nach Stellenprozenten, Erfahrung im Amt, weiteren Funktionen etc. Während meiner Forschungsaufenthalte wurde in den meisten Fällen bei einer 100-Prozent-Stelle ein 〉Output` von ca. 18 bis 20 Entscheiden und vier bis fünf Anhörungen pro Monat verlangt. 
müssen in viereinhalb Stunden herausfinden, aufgrund einer Anhörung, ob etwas glaubhaft ist oder nicht.« (Interview T, meine eigene Übersetzung ${ }^{15}$ )

In Bezug auf die Einschätzung der begründeten Furcht vor zukünftiger Verfolgung wird durch institutionalisierte Praxis(-regeln) versucht, Wahrscheinlichkeitseinschätzungen vorweg zu nehmen. Dies geschieht einerseits dadurch, dass vergangene Verfolgung (sofern sie als glaubhaft und >aktuell beurteilt wird) als ein Indiz möglicher, zukünftiger Verfolgung gedeutet wird und andererseits durch die in den APPAs aufgeführten »Risikoprofile«. Die sogenannten Risikoprofile definieren Gruppen von Personen, von denen man annimmt, dass sie bei einer Rückkehr in ihr Heimatland verfolgt werden könnten. Je nach Land und Kontext können verschiedene Kriterien ausschlaggebend sein, ob man einer solchen Risikogruppe zugerechnet wird oder nicht. Solche Kriterien können beispielsweise Parteimitgliedschaften sein, der Wohnort vor der Ausreise, die Zugehörigkeit zu einer religiösen Gemeinschaft, das Alter etc. Für die Entscheidungspraxis bedeutet dies, dass der Bestimmung solcher Gruppenzugehörigkeiten eine wichtige Rolle zukommt.

Sowohl bestimmte Gruppenzugehörigkeiten als auch geltend gemachte vergangene Verfolgung werden jedoch nur dann als Indiz für zukünftige Verfolgung erachtet, wenn sie als glaubhaft bewertet werden. Die Glaubhaftigkeitsbeurteilung bzw. die rechtliche Argumentation mit dem Glaubhaftigkeitsartikel spielt insbesondere dann eine wichtige Rolle, wenn die Sachbearbeitenden für die Einschätzung der begründeten Furcht vor zukünftiger Verfolgung keine (>genauen`) Risikoprofile zur Verfügung haben. Je weniger man über ein Land wisse bzw. je willkürlicher ein Land funktioniere, desto schwieriger würde die Beurteilung der Gefahr durch zukünftige Verfolgung, wurde mir von Sachbearbeitenden mehrmals erklärt. In solchen >Fällen` sei es einfacher, mit dem Glaubhaftigkeitsartikel zu argumentieren:

»Zum Beispiel eine Mafia-Geschichte. Ist das asylrelevant oder nicht? [...] Weil [...] der Staat ist nicht unbedingt schutzfähig und es steckt zwar irgendwie kein Asylmotiv dahinter, aber [der Gesuchsteller] ist doch irgendwie einer Verfolgung ausgesetzt. Aber wenn du jetzt sagst, das alles ist unglaubhaft, dann sicherst du dich doch irgendwie ab." (Interview $\mathrm{R}$ )

15 Dies gilt auch für alle weiteren hier verwendeten Aussagen von SEM Sachbearbeitenden. Ich habe sowohl Aussagen aus dem Schweizerdeutschen als auch aus dem Französischen übersetzt. 
Im schweizerischen Asylverfahren wird die Mehrheit der Negativentscheide mit Unglaubhaftigkeit begründet. ${ }^{16}$ Dass die Glaubhaftigkeitsprüfung eine zentrale Rolle spielt, ergibt sich einerseits aus den rechtlichen Grundlagen. Die Grafik oben zeigt, dass die Glaubhaftigkeit von Asylvorbringen die Voraussetzung dafür ist, dass Gesuchstellende als Flüchtlinge anerkannt werden. Andererseits müsste die Glaubhaftigkeitsbeurteilung für negative Entscheide rechtlich gesehen nicht immer eine wichtige Rolle spielen. Für negative Entscheide reicht es nämlich, wenn nur eine der beiden Fragen »Sind die Vorbringen glaubhaft? « und »Erfüllt die Person die Flüchtlingseigenschaft?« mit »nein« beantwortet wird. Trotzdem wird in der Praxis meist mit dem Glaubhaftigkeitsartikel argumentiert und nicht mit dem Flüchtlingsbegriff. ${ }^{17}$ Meine These ist, dass dies einerseits mit dem verfahrensinhärenten Misstrauen zusammenhängt und andererseits damit, wie im Asylverfahren Entscheidungswissen hergestellt wird.

\section{Institutionelles Misstrauen}

»Aufgabe des Asylverfahrens ist es, unter den neu eintreffenden Asylsuchenden jene zu erkennen, die nach den beschriebenen Kriterien Anspruch auf Schutz haben. Viele Asylsuchende sind weder Flüchtlinge noch Kriegsvertriebene. [...] Sie suchen in der Schweiz einen besseren Platz zum Leben. [...] Für die Befragung durch die Behörden legen sich manche von ihnen eine dramatische Verfolgungsgeschichte zu. Sie hoffen, dadurch den Flüchtlingsstatus zu erlangen. Aus der Sicht des Betroffenen ist dieses Verhalten verständlich, aus asylrechtlicher Perspektive handelt es sich um einen Missbrauch des Asylverfahrens. Die Behörden müssen solche Gesuche möglichst rasch abweisen und die Wegwei-

16 Statistiken dazu, ob negative Entscheide alleine aufgrund von Artikel 7 oder von Artikel 3 AsylG bzw. durch eine Kombination von beiden begründet wurden, gibt es nicht, da solche Entscheide im System mit dem gleichen Code versehen werden. Doch im Empfinden aller meiner Gesprächspartner_innen kommen Artikel-7-Entscheide viel häufiger vor als reine Artikel-3-Entscheide. Auch im Handbuch »Asyl und Rückkehr« des SEM steht: »Die Mehrzahl der Ablehnungen von Asylgesuchen ist auf die fehlende Glaubhaftigkeit der Vorbringen der asylsuchenden Personen zurück zu führen.« (Staatssekretariat für Migration [SEM] o.J.: 7) Mein Dossier-Sample zeigt, dass von den 39 Negativentscheiden, die ich analysiert habe, in 27 Fällen unter anderem mit Artikel 7 AsylG argumentiert wurde.

17 Dies entspricht der Amtspraxis, zu der die Sachbearbeitenden angeleitet werden. 
sung konsequent vollziehen. Dadurch wird das Asylverfahren für arbeitsuchende AusländerInnen unattraktiv. ${ }^{18}$

Dies schreibt das SEM unter »Kernpunkte der Eidgenössischen Asylpolitik« auf seiner Webseite. Wir erkennen in diesem Auszug die Funktion der Sachbearbeitenden als »gatekeepers« oder »guardians of a common good « (Heyman 2009: 381; Hoag 2011: 82; Lipsky 2010: 4). Das »common good « ist in diesem Fall eine (vorläufige) Aufenthaltsberechtigung in der Schweiz. Sachbearbeitende können es vergeben, müssen es aber auch vor Missbrauch schützen. Des Weiteren erkennen wir auch die Annahme, dass viele der Asylsuchenden nicht schutzbedürftig seien und bei der Anhörung lügen würden, um Schutz zu erhalten. Diese Lügen müssten durch das Verfahren aufgedeckt werden. Viele Wissenschaftler_innen, die sich mit Asyl- und Immigrationsverfahren beschäftigen, beschreiben denn auch, dass Entscheidungsfindungen häufig von Misstrauen begleitet werden (vgl. Alpes/Spire 2014; Fassin/D’Halluin 2005; Kelly 2012). So wird Misstrauen - quasi als die Umkehrung von Naivität - als ein Zeichen von Professionalität gesehen (vgl. Alpes/Spire 2014: 269). ${ }^{19}$ Die Annahme, dass viele Asylgeschichten konstruiert seien, führt dazu, dass Anhörungen in der Praxis zu einer Suche nach (Un-)Wahrheiten werden (vgl. Anderson et al. 2014; Jubany 2011; Macklin 1998; Souter 2011).

Im Gegensatz zum Strafverfahren, in dem zunächst die Unschuldsvermutung gilt, geht es deshalb in Asylanhörungen darum, so lange zu fragen, bis einen die gesuchstellende Person von der >Wahrheit` der Geschichte überzeugt hat oder bis man genügend Argumente für einen negativen Asylentscheid hat, mit denen man wiederum andere überzeugen kann - in erster Linie die sektionsleitende Person und im Fall einer Beschwerde das Bundesverwaltungsgericht:

»A: [...] Und dann lasse ich die Person erzählen und prüfe die Glaubwürdigkeit.

F: In jedem Fall?

A: Ja, eigentlich schon. Also, das ist nicht, um böse zu sein, nicht, dass ich jetzt davon ausgehe, dass sie sowieso lügen, das nicht. Aber man möchte halt wissen, ob sie es wirklich erlebt haben. Und was sie davon wissen. [...] Teilweise merkt man schnell, dass es stimmt. [...] Dann hake ich zum Teil nicht mehr nach, man muss sie ja nicht noch mehr belasten. Und wenn es mir nicht glaubwürdig erscheint, dann hake ich

18 https://www.bfm.admin.ch/bfm/de/home/asyl/asyl/asylrecht.html vom 16.10.15.

19 Im SEM höre ich oft, dass man, wenn man neu im Amt sei, halt noch ein bisschen zu naiv sei - eine solche Naivität wird auch mir zugeschrieben. Erst die Erfahrung lehre einen, nicht mehr alles >einfach so zu glauben. 
nach, bis ich entweder genügend Argumente habe für meinen Entscheid, genügend Widersprüche, oder bis er mich überzeugt hat, dass es doch stimmt.« (Interview A)

Die Aussagen des Sachbearbeiters verdeutlichen, dass nicht alle Geschichten per se in Zweifel gezogen werden - man kann auch schnell >merken`, dass etwas stimmt. Aber man muss es eben zuerst >merken $<$ und dies bedeutet, dass quasi in jedem Fall eine Umkehrung der Unschuldsvermutung stattfindet. Denn anders kann die Glaubhaftigkeit gar nicht geprüft werden. Es ist eine Prüfung, die es selbst im Vertrauensfall braucht, denn es kann nur dann positiv entschieden werden, wenn man die nötigen Argumente dazu hat. Und die müssen erst produziert werden.

\section{Bürokratische Entscheidungslogik und die Herstellung von Entscheidungswissen}

»Entscheiden, das ist unser Handwerk. [...] Das ist unser Kerngeschäft [...]. Das heißt, wir sind im Prinzip wie Cäsar in der Arena, der so macht [Daumen hoch] oder so macht [Daumen runter], wir sagen ja oder nein. Es gibt noch so gewisse Grautöne dazwischen, aber letztlich sagen wir ja oder nein, das ist der Entscheid. Und der Entscheid muss so gewissen Kriterien entsprechen. [Kriterien], die man auflisten kann, die sich aus Recht und Praxis ergeben und dann hat es irgendwo dazwischen immer noch so das Bauchkriterium.«(Interview V)

Die Sachbearbeiterin spricht in diesem Zitat drei Punkte an, die ich in diesem Teil des Artikels diskutiere. Erstens beschreibt die Sachbearbeiterin die Form von Entscheiden: jeder Entscheid muss eindeutig mit »ja» oder »nein« beantwortet werden. Zweitens wird klar, dass jeder Asylentscheid mit Gesetzeskriterien begründet werden muss. Drittens spricht die Sachbearbeitende die Rolle des >Bauchgefühls` für das Fällen von Asylentscheiden an. Ersteres verbinde ich mit der von Handelman definierten »bürokratischen Logik«. Auf die letzten beiden Punkte gehe ich im Zusammenhang mit der Herstellung von Entscheidungswissen ein.

Handelman definiert bürokratische Logik als die Schaffung linearer Formen der Klassifikation (2004: 6). Ohne solche Formen der Klassifikation könnten Bürokratien gar nicht bestehen (vgl. Handelman 1995: 280). Die taxonomischen Klassifikationsschemata, die Handelman beschreibt, bestehen aus Kategorien, die hierarchisch angeordnet sind und sich eindeutig voneinander abgrenzen. Entweder gehört etwas in eine Kategorie oder es wird eindeutig davon ausge- 
schlossen. Auf einem solchen Schema beruht auch das decision-making im SEM, wie man der Grafik im vorhergehenden Teil entnehmen kann. Die Grafik zeigt die vier Rechtskategorien, denen die Gesuchstellenden durch das Verfahren eindeutig zugeordnet werden müssen. Die Zuordnung geschieht über eine Reihe hierarchisch angelegter Fragen, die alle eindeutig mit »ja« oder »nein« beantwortet werden müssen. Eine Antwort dazwischen gibt es nicht.

Es reicht jedoch nicht aus, »Ja-« oder »Nein-« Entscheide zu treffen. Jede Entscheidung muss auch begründet werden. Bei negativen Entscheiden geschieht dies verfahrensöffentlich; Gesuchstellende erhalten ein Schreiben mit den Gründen für den negativen Asylentscheid. Positive Entscheide hingegen werden nur intern begründet. Damit will man verhindern, dass Gesuchstellende lernen, wie man ein erfolgreiches Asylgesuch stellt. Sowohl positive als auch negative Asylentscheide werden dadurch begründet, dass Gesetzeskriterien mit aktenkundigen Tatsachen verknüpft werden. ${ }^{20}$ Im Verlauf des Verfahrens müssen also erstens aktenkundige Tatsachen und zweitens Verknüpfungen mit dem Gesetz hergestellt werden. Das Wissen, das zu diesem Zweck generiert wird, nenne ich Entscheidungswissen.

Entscheidungswissen ist jenes Wissen, das zu einem Endprodukt, dem fertigen Asylentscheid, führt. Es ist charakteristisch für die bürokratische Verwaltung. Ich argumentiere, dass Entscheidungswissen durch das Zusammenspiel von Fach- und Dienstwissen generiert wird. Unter Fachwissen verstehe ich im untersuchten Kontext die Länder- und Gesetzeskenntnisse von Sachbearbeitenden. $^{21}$ Dienstwissen bezieht sich, wie Weber (2005: 165f.) schreibt, auf aktenkundige Tatsachenkenntnisse, die durch das Verfahren erzeugt werden. Im Asylverfahren sind dies alle Schriftstücke, die im Dossier abgelegt werden: die auf jeder Seite mit einer Unterschrift der asylsuchenden Person bestätigten Anhörungsprotokolle, materielle Beweise, Gutachten von sogenannten Expertinnen und Experten etc. Über Weber (ebd.) hinausgehend argumentiere ich, dass in bürokratischen Entscheidungsprozessen noch eine weitere Form von Dienstwissen existiert und zur Generierung von Entscheidungswissen beiträgt: professionelles Wissen. Unter professionellem Wissen verstehe ich das, was die Sachbearbei-

20 In der Praxis geschieht dies in beide Richtungen, wie ich im Folgenden aufzeige. Sachbearbeitende gehen teilweise von Tatsachen aus und suchen nach passenden Gesetzeskriterien oder sie gehen umgekehrt von bestimmten Gesetzeskriterien aus und suchen im Asyldossier nach passenden Tatsachen.

21 Mit Gesetzeskenntnis meine ich sowohl die Kenntnis relevanter Gesetzesartikel, die für Asylentscheide verwendet werden, als auch die Kenntnis formalisierter institutioneller Praxisregeln, wie man sie vor allem in den APPAs findet. 
tenden selbst jeweils als >Bauchgefühl bezeichnen. Professionelles Wissen ist jedoch nicht nur eine Intuition, wie das Wort >Bauchgefühl« suggeriert. Vielmehr ist es geprägt durch das Fachwissen, das Erfahrungswissen und die Entscheidungsroutinen, welche die Sachbearbeitenden erarbeitet haben.

Im Folgenden zeige ich zunächst am Beispiel der Herkunftsbestimmung die Verknüpfung von Länderwissen und aktenkundigen Tatsachen auf. Danach lege ich anhand des Entscheidungskriteriums »Widersprüche« dar, wie im und durch das Asylverfahren aktenkundige Tatsachen hergestellt werden und mit Recht in Verbindung gebracht werden. Schließlich untersuche ich, welche Rolle professionelles Wissen für die Generierung von Entscheidungswissen spielt. Ich zeige auf, dass professionelles Wissen eine richtungsweisende Funktion hat, jedoch im fertigen Asylentscheid unsichtbar gemacht wird.

\subsection{Länderkenntnisse für die Herkunftsprüfung}

Die Ermittlung der (sozialen) Herkunft von Gesuchstellenden gehört zu den ersten Schritten im Asylverfahren und ist richtungsweisend für das weitere Vorgehen. In erster Line gilt es dabei ’herauszufinden<, aus welchem Land der/die Gesuchsteller_in kommt bzw. in welchem Land er oder sie sogenannt hauptsozialisiert wurde. Aber auch andere biographische Merkmale, wie zum Beispiel das Alter der Person, ihr Bildungsgrad, ihr genauer Wohnort im Herkunftsland etc. werden über das Verfahren ermittelt. All diese `Herkunftskriterien` sind entscheidend für die Zuordnung zu bestimmten Risikoprofilen (oder umgekehrt für den Ausschluss aus bestimmten Risikoprofilen), sowohl in Bezug auf die Flüchtlingseigenschaft als auch auf den Vollzug der Wegweisung.

Am einfachsten und schnellsten geht die Herkunftsbestimmung anhand von Identitätsdokumenten. ${ }^{22}$ Da materielle Beweise oft fehlen, wird in der Praxis versucht, die Herkunft von Gesuchstellenden zu eruieren anhand von Fragen nach dem, was Scheffer die »Mitgliedschaftskompetenz« nennt (Scheffer 2001: 146f.). ${ }^{23}$ Anhand dieser Fragen soll bestimmt werden, ob die von den Gesuchs-

22 Wenn Identitätsdokumente eingereicht werden, gibt es für die Sachbearbeitenden drei Handlungsmöglichkeiten: Sie können die Dokumente als echt anerkennen, sie können die Dokumente auf ihre Echtheit hin überprüfen lassen oder sie können die Beweisdokumente als untauglich bewerten. Letzteres kommt beispielsweise dann zur Anwendung, wenn Gesuchstellende Kopien von Identitätsdokumenten einreichen, insbesondere wenn diese nicht sie selbst, sondern zum Beispiel Familienmitglieder betreffen.

23 In den 50 bisher von mir analysierten Dossiers wurden in 16 Fällen originale Identitätsdokumente eingereicht. In vier Fällen haben die Sachbearbeitenden ein Dokumen- 
tellenden angegebene Herkunft >stimmt oder nicht . Die Herkunftsprüfung wird in der Regel in der Befragung zur Person in den Empfangs- und Verfahrenszentren durchgeführt. Gefragt wird danach, was jemand, der oder die aus einem bestimmten Land oder Ort kommt, >wissen müsste`, wie man in diesem Auszug aus einem BzP-Protokoll erkennt:

$\gg$ F: Wie lautet die Telefonvorwahl von Marokko?

A: 00212 .

F: Wie heißt der König von Marokko?

A: (GS lächelt) Das ist Mohammed der VI. Ich bin ein echter Marokkaner.

F: Wie heißt seine Frau, die Königin?

A: Ich weiß, dass sie aus Fes stammt. Als er geheiratet hat, war ich nicht in Marokko.

F: Wie heißen die umliegenden Ortschaften [von] Kneitra?

A: Kneitra liegt ca. $40 \mathrm{~km}$ von Rabat entfernt. In Kneitra gibt es einen amerikanischen Stützpunkt. Die umliegenden Ortschaften heißen Sidi Yahia, Sidi Suleiman und Sidi Kassem.« (Auszug aus einem BzP Protokoll)

In diesem Fall hat der Gesuchsteller die Herkunftsprüfung bestanden; er hat seine Mitgliedschaftskompetenz bewiesen. Die Sachbearbeiterin hat die Antworten des Gesuchstellers anhand ihres eigenen Länderwissens bewertet. Ihr Länderwissen hat sie sich selbst angeeignet: durch das Lesen von internen Länderreports, durch das, was sie von anderen Gesuchstellenden in der Anhörung erfahren hat, durch eigene Internetrecherchen, das Studieren von Landkarten etc. Wenn die Sachbearbeitenden nach der Prüfung der Mitgliedschaftskompetenz die Herkunft von Asylsuchenden immer noch anzweifeln, kann eine Lingua-Analyse in Auftrag gegeben werden, die den Sozialisierungsort der Gesuchstellerin oder des Gesuchstellers ermitteln soll. ${ }^{24}$ Manchmal stellen die Sachbearbeitenden auch Botschaftsanfragen und bitten die Leute vor Ort, zu ermitteln, ob eine Person

tengutachten erstellen lassen; die Echtheit des Dokumentes wurde in allen vier Fällen bestätigt.

24 Lingua-Analysen werden auf der Webseite des SEM folgendermaßen definiert: »LINGUA ermittelt mit Hilfe von externen, unabhängigen Experten den Sozialisationsraum der Gesuchsteller und hält die Resultate der Ermittlungen in einem Gutachten fest. Die LINGUA-Gutachten basieren auf einer linguistischen Analyse der Sprache und auf einer landeskundlich/kulturellen Analyse des Wissens der asylsuchenden Person.« (https://www.bfm.admin.ch/bfm/de/home/asyl/asylverfahren/drei_beispiele/ analysen.html vom 21.04.15) 
beispielsweise tatsächlich im angegeben Quartier gelebt hat. ${ }^{25}$ Die LinguaGutachten verkörpern sowohl Fachwissen als auch Dienstwissen. Sie basieren auf dem Länder- und Sprachwissen von sogenannten Expertinnen und Experten. Gleichzeitig gelten Gutachten wie auch Botschaftsantworten als aktenkundige Tatsachen, die für Entscheidungsbegründungen verwendet werden können.

\subsection{Widersprüche als aktenkundige Tatsachen}

Meine Analyse der Asyldossiers zeigt, dass Widersprüche das meist verwendete Unglaubhaftigkeitskriterium in negativen Asylentscheiden sind: so wurde in 21 von 27 >Unglaubhaftigkeitsentscheiden` die Unglaubhaftigkeit unter anderem mit dem Kriterium »Widersprüche« begründet. In Anbetracht dessen, dass die Entscheider_innen im SEM unter hohem Zeitdruck arbeiten, argumentiere ich, dass die häufige Verwendung des Kriteriums »Widersprüche« damit zusammenhängt, dass solche Argumente einfach und schnell zu produzieren sind und keiner weiteren Abklärungen (wie zum Beispiel Botschaftsanfragen) bedürfen. Denn Widersprüche >findet man in den Protokollen, sie werden durch die Anhörungen selbst produziert.

Die häufige Verwendung des Kriteriums drückt sich auch in dessen Beliebtheit aus. In meinen Interviews habe ich den Sachbearbeitenden Karteikarten gegeben, auf denen die Kriterien aus den Textbausteinen für negative Entscheide mit Artikel 7 AsylG standen. ${ }^{26}$ Ich habe die Sachbearbeitenden gebeten, die Karten in eine Rangordnung zu bringen; beginnend mit dem Kriterium, das sie wenn möglich - am liebsten für die Begründung von Unglaubhaftigkeit verwenden, absteigend bis zum Kriterium, das sie am wenigsten gerne verwenden. Im Anschluss daran habe ich mit den Sachbearbeitenden über ihr Ranking gespro-

25 Wie genau die Mitgliedschaftskompetenz geprüft wird, also wie viele und wie detaillierte Fragen dabei gestellt werden, und ob noch weitere Abklärungen wie LinguaAnalysen oder Botschaftsanfragen getätigt werden, hängt von verschiedenen Faktoren ab. Eine wichtige Rolle spielt dabei das angegebene Herkunftsland selbst. Zum Teil bestehen institutionelle Vorgaben betreffend der Herkunftsbestimmung von Asylsuchenden aus bestimmten Ländern. In anderen Fällen wissen die Sachbearbeitenden aus Erfahrung, wie genau die Herkunft zu prüfen ist.

26 Für die Argumentation in Negativentscheiden mit Artikel 7 AsylG stehen den Sachbearbeitenden Textbausteine zu folgenden Kriterien zur Verfügung: gefälschte Beweismittel, mangelnde Substantiierung, nachgeschobene Vorbringen, nicht mehr geltend gemachte Vorbringen, Tatsachenwidrigkeit, untaugliche Beweismittel, widersprechen der allgemeinen Erfahrung, widersprechen der Logik des Handelns, Widersprüche. 
chen. Die allermeisten Sachbearbeitenden haben das Kriterium »Widersprüche« an die erste oder zweite Stelle gesetzt (hinter »Tatsachenwidrigkeit «). ${ }^{27}$ Begründet wurde dies meist damit, dass Entscheide mit Widersprüchen einfach seien und dass man diese gut belegen könne, da man Fakten habe, genauso wie bei gefälschten Beweisen oder Tatsachenwidrigkeiten. ${ }^{28}$

Die Argumentation mit Widersprüchen basiert auf dem Aussagenabgleich. Dabei werden Vergleiche zwischen verschiedenen Aussagen gezogen: zwischen den Aussagen, welche die gesuchstellende Person in der BzP gemacht hat und denen in der Anhörung, zwischen verschiedenen Aussagen in der Anhörung selbst sowie zwischen Aussagen verschiedener Personen, die verwandt oder zusammen geflüchtet sind. Die Grundannahme für solche Vergleiche ist, dass eine Person, die etwas erlebt hat, bzw. mehrere Personen, die das Gleiche erlebt haben, immer wieder das Gleiche erzählen würden. Das Verfahren an sich bietet dadurch, dass jede_r Asylsuchende mindestens zweimal befragt wird, und dass verwandte Personen, wie zum Beispiel Ehepartner, getrennt voneinander befragt werden, mehrere Abgleich-Möglichkeiten. Andere Abgleich-Möglichkeiten entstehen durch das Vorgehen in den Anhörungen und die Fragen, die dabei gestellt werden.

Obwohl es keinen Fragenkatalog für Anhörungen gibt und die Befrager_innen ihre eigenen Fragen entwickeln, sind das Vorgehen in den Anhörungen und die Fragen, die gestellt werden, meist sehr ähnlich. ${ }^{29}$ Die Anhörung beginnt nach einer kurzen Einführung, in der die Anwesenden (in ihrer Funktion) vorgestellt werden und die gesuchstellende Person auf ihre Rechte und Pflichten hingewiesen wird, mit der Frage nach Beweisdokumenten. Danach folgen häufig Fragen zur Familie und zum Leben im Herkunftsland. Der nächste Teil zu den Asylgründen beginnt meist mit einer oder mehreren offenen Fragen, wie zum

27 Während die meisten meiner Gesprächspartner_innen »Widersprüche« als eines der besten und einfachsten Kriterien bewerteten, fanden andere, dass die Argumentation mit Widersprüchen problematisch sei, weil es kaum möglich sei, eine Geschichte zweimal gleich zu erzählen. Sie setzten das Kriterium deshalb im Ranking auf eine der hintersten Stellen und kritisierten die häufige Verwendung des Kriteriums in Asylentscheiden.

28 Siehe Fußnote 12.

29 Dies hat wohl unter anderem damit zu tun, dass die Sachbearbeitenden voneinander lernen, welche Fragen man stellen könnte. Dies insbesondere beim Anfangscoaching, bei dem sie Anhörungen von anderen beiwohnen. Aber auch später haben sie immer wieder mal mit Protokollen anderer Befragungen zu tun. Des Weiteren lassen sich in der Maske für das Anhörungsprotokoll und in den APPAs Beispielfragen finden. 
Beispiel »Weshalb haben Sie (Land XY) verlassen und in der Schweiz Asyl beantragt?«, gefolgt von gezielten W-Fragen und geschlossenen Fragen. Zum Schluss werden die Gesuchstellenden meist mit sogenannten Widersprüchen konfrontiert. Dieses Vorgehen vergleichen viele Entscheider_innen mit einem Trichter oder dem Zuziehen einer Schlinge. So geht es bei Anhörungen oft darum zu testen, ob sich die gesuchstellende Person in Widersprüche >verstrickt oder nicht<.

Sachbearbeitende erachten sowohl das Stellen von offenen Fragen als auch das Stellen von zusammenhängenden Fragen als hilfreiche Strategien, um Widersprüche raufzudecken`. So erklärten mir einige Sachbearbeitende, dass sich die Gesuchstellenden - sofern die Geschichte nicht >wahr s sei - viel eher >in Widersprüche verstricken` würden, wenn man zuerst eine offene Frage stelle und die Gesuchstellenden dann lange und frei erzählen lasse. Das Gleiche passiere auch, wenn man sich-aufeinander-beziehende Fragen stelle, denn diese könnten die Gesuchstellenden oft nicht widerspruchslos beantworten. Durch solche Fragestrategien werden aktenkundige Tatsachen erzeugt, die sich mit dem Kriterium "Widersprüche« verbinden lassen. Wie dies in der Praxis geschieht, stelle ich anhand >eines Falles` des Sachbearbeiters Bernard dar. ${ }^{30}$

Es handelt sich dabei um ein Gesuch eines jungen Eritreers, der als Asylgrund geltend machte, aufgrund einer unterstellten Beziehung zu einem Regimekritiker zweimal von Soldaten zuhause aufgesucht worden zu sein: Einmal hätten die Soldaten ihn befragt und geschlagen, beim zweiten Mal sei sein Haus durchsucht worden. Daraufhin habe er seine Heimatstadt Stadt Barentu verlassen. Bernard hat das Gesuch abgelehnt und den Entscheid hauptsächlich mit dem Kriterium »Widersprüche« begründet. ${ }^{31}$ Viele der von ihm im Entscheid aufgeführten Widersprüche beziehen sich auf Zeitangaben und die chronologische Abfolge von Geschehnissen:

»Während Sie [der Entscheid ist direkt an den Gesuchsteller gerichtet] in Ihrer freien Erzählung vorbringen, zwischen der ersten behördlichen Befragung und der Hausdurchsuchung durch das Militär seien 20 Tage bis ein Monat vergangen [vgl. Akte X, S. Y], machen Sie später geltend, zwischen der ersten behördlichen Befragung und der Hausdurch-

30 Aus Anonymisierungsgründen habe ich verschiedene Identifikationsmerkmale des Sachbearbeiters und der gesuchstellenden Person (Name, Herkunftsland, Geschlecht etc.) geändert. Auch die Auszüge aus dem Asylentscheid und den Anhörungsprotokollen habe ich abgeändert.

31 Der Mann erhält jedoch eine vorläufige Aufnahme, da er aufgrund seines militärtauglichen Alters einem Risikoprofil im APPA entspricht. 
suchung seien lediglich zwei bis drei Tage vergangen [vgl. Akte XX, S. Y]. Gleich darauf sagen Sie zudem aus, Ihr Haus sei einige Wochen nachdem Sie aufgefordert worden seien in den Militärdienst einzurücken, durchsucht worden [vgl. Akte XX, S. Z ].« (Auszug aus dem Asylentscheid)

Bernard ist über die Anhörung zu diesem Argument gekommen. Wie üblich hat Bernard den Anhörungsteil zu den Asylgründen mit einer offenen Frage begonnen: »Gut, dann können Sie mir jetzt frei erzählen, was Ihnen persönlich in Eritrea geschehen ist und warum Sie Eritrea dann verlassen haben.« (Auszug aus dem Anhörungsprotokoll) In seiner Antwort sagte der Gesuchsteller unter anderem:

»Es kamen etwa zwei, drei bewaffnete Personen zu mir. Sie haben mir Fragen gestellt. [...] Diese Leute haben mich dann verbal beleidigt. Dann haben sie mich geschlagen. Was danach passierte, weiß ich nicht. [...] Man ließ uns etwa 20 Tage bis einen Monat in Ruhe. [...] Die Soldaten haben dann unser Haus durchsucht.« (Auszug aus dem Asylentscheid)

Später in der Anhörung begann Bernard dem Gesuchsteller geschlossene Fragen und gezielte W-Fragen zu stellen. Darunter waren auch solche, welche die Befragung und die Hausdurchsuchung durch die Soldaten betrafen:

$» F$ : Nur eine kleine Frage zur Bestätigung: Wenn ich Sie richtig verstanden habe, kamen die Behörden zweimal zu Ihnen nach Hause, das erste Mal wurden Sie befragt und man hat Sie geschlagen und beim zweiten Mal wurde das Haus beschlagnahmt. Stimmt das so?

A: Ja. Sie haben gewusst, dass ich Kontakt zu X hatte. Die Regierung hat überall Spione.

F: Wie viel Zeit ist zwischen diesen Ereignissen etwa vergangen?

A: Kurze Zeit, etwa zwei, drei Tage. Man wollte ja von mir unbedingt wissen, was mir X gesagt hatte.

F: Können Sie sich auch noch an das Datum erinnern, an dem Ihr Haus beschlagnahmt wurde und Sie Barentu verlassen haben?« (Auszug aus dem Befragungsprotokoll)

Und schließlich sprach er den Gesuchsteller am Ende der Anhörung auf einzelne Widersprüche an:

$» \mathrm{~F}$ : Sie haben heute ausgesagt, nach der ersten behördlichen Befragung hätten Sie etwa 20 Tage Ruhe gehabt. Später haben Sie dann gesagt, zwischen den zwei Befragungen seien zwei oder drei Tage vergangen.

A: Ja. 
F: Was stimmt denn jetzt?

A: Es waren etwa zwei oder drei Tage. Wenn ich das letzte Mal von etwa 20 Tagen sprach, dann war damit die Zeitspanne gemeint, als ich Barentu verlassen habe. Es gab viele Probleme, deshalb kann ich mich nicht mehr an alles genau erinnern.« (Auszug aus dem Anhörungsprotokoll)

Am Ende der Anhörung war Bernard überzeugt, dass diese (und andere) Aussagen des Gesuchstellers unglaubhaft waren. Der Gesuchsteller hatte ihn nicht vom Gegenteil überzeugen können. Der >Fall endete damit so, wie es Bernard schon nach dem Lesen des BzP-Protokolls vermutete hatte. Denn Bernard waren bereits beim Lesen des Protokolls ein paar >Unstimmigkeiten` aufgefallen. Wie dies üblich ist, hatte er die Anhörung deshalb in eine bestimmte Richtung vorbereitet und im Vorfeld Fragen notiert, von denen er glaubte, dass sie - sollte die Geschichte tatsächlich unglaubhaft sein - aktenkundige Widersprüche erzeugen würden. Letztlich ist genau das geschehen.

\subsection{Von professionellem Wissen zu Entscheidungswissen}

Wie Bernard wissen Entscheider_innen meist schon früh, wie der Entscheid ausfallen wird. Die Schwierigkeit liegt denn auch nicht darin, Entscheidungen zu fällen, sondern diese zu begründen. Erst mit der Begründung ist das Entscheidungswissen komplett. Erst damit kommt man nämlich zu einem Endprodukt, dem fertigen Asylentscheid. Dass die Entscheidungsfindung an sich von den Sachbearbeitenden als einfach betrachtet wird, hat mit professionellem Wissen zu tun.

Professionelles Wissen trägt zur Herstellung von Entscheidungswissen bei, indem es die Richtung vorgibt (oder zumindest mitbestimmt), in welche befragt wird. ${ }^{32}$ Bernard zum Beispiel hat Fragen vorbereitet, die auf Widersprüche abzielen. Umgekehrt war ich ein anderes Mal bei einer Anhörung dabei, in welcher die Sachbearbeiterin gänzlich auf die Glaubhaftigkeitsprüfung verzichtete, weil sie nach dem Lesen des BzP-Protokolls bereits überzeugt war, dass die Geschichte glaubhaft sei. Als ich sie fragte, warum dies für sie so klar sei, meinte sie: »Ich schaue mir das Protokoll an und ich weiß es.« (Auszug aus dem Forschungstagebuch) Außerdem hätte der Gesuchsteller sehr detailliert und in einer logischen und chronologischen Weise gesprochen. Und er hätte Dinge gesagt, die auf sein Heimatland zutreffen würden.

32 Die Richtung kann sich im Verlauf des Verfahrens auch ändern. 
Dass professionelles Wissen eine wichtige Bedeutung hat für die Richtungsbestimmung von Entscheiden, zeigt sich auch darin, dass Sachbearbeitende es bevorzugen, Entscheidungen zu treffen, wenn sie die Person selbst angehört haben. Einerseits, weil sie selbst die Fragen stellen und so einfacher Argumente für den Entscheid generieren können. Anderseits empfinden es viele als hilfreich, die asylsuchende Person selbst zu sehen und zu erleben. Man bekomme dann einen >persönlichen Eindruck` von der Person. Der `persönliche Eindruck ‘ bezieht sich nicht nur auf die verbalen Aussagen der asylsuchenden Person, sondern auch auf deren non-verbales Verhalten während der Anhörung. Zwar wird mir von den Sachbearbeitenden in den Interviews jeweils entsprechend der offiziellen Norm gesagt, das Verhalten und die gezeigten Emotionen der Gesuchstellenden seien vollkommen irrelevant. Wenn ich jedoch mit Sachbearbeitenden, zum Beispiel während Anhörungspausen, konkrete `Fälle ২ bespreche, wird häufig auf Verhalten und Emotionen der Gesuchstellenden Bezug genommen, um Entscheidungen oder erste Ideen einer Entscheidungsrichtung zu begründen.

»Es ist mehr wie ein Gefühl. Und nachher suche ich im Text danach« (Interview U), erklärte mir ein Sachbearbeiter sein Vorgehen bei der Glaubhaftigkeitsbeurteilung. Diese Aussage verdeutlicht, dass professionelles Wissen nicht nur die Richtung mitbestimmt, in die befragt wird, sondern auch die Richtung, in welcher während des Schreibprozesses nach Argumenten gesucht wird. Gesucht wird dabei vor allem in den Anhörungsprotokollen. Das folgende Beispiel von Sachbearbeiterin Manuela zeigt dies deutlich ${ }^{33}$ :

»Ich sitze neben Manuela an ihrem Pult und schaue ihr beim Verfassen des Entscheides zu. Es geht um eine Frau aus Sri Lanka, und eigentlich steht der Entscheid für Manuela bereits fest: die Frau bekommt kein Asyl, aber eine vorläufige Aufnahme. Für Manuela ist >der Fall klar unglaubhaft, denn die Vorbringen der Frau sind ihrer Auffassung nach völlig unsubstantiiert. Gleichzeitig bemerkt Manuela, dass die Gesuchstellerin auf jede Nachfrage hin etwas mehr hinzufügt. Eine reine Argumentation mit Unsubstantiiertheit findet sie deshalb schwierig. Manuela bedauert, dass in den Aussagen der Gesuchstellerin keine Widersprüche drin sind, denn Widersprüche würden die Argumentation von Unglaubhaftigkeit viel einfacher machen. Zur Sicherheit hat sich Manuela deshalb noch das Protokoll des Bruders der Frau angeschaut. Einfach um zu schauen, ob es ihr Gefühl bestätigt oder nicht. In diesem Fall wurde es bestätigt. Manuela sagt mir, dass es wohl einen kombinierten Entscheid [Entscheid, in welchem mit Artikel 3 und Artikel 7 AsylG argumentiert wird] geben werde, denn mit der Unglaubhaftigkeit alleine hätte sie zu wenig. Aber vielleicht, meint sie, komme es ja dann beim Schreiben ganz anders.

33 Siehe Fußnote 30. 
Vor ihrem Computer sitzend, beginnt sie nun, den Sachverhalt aufzuschreiben. Dann wählt sie zwei Textbausteine aus, einen zu Artikel 3 und einen zu Artikel 7 AsylG, nämlich den der mangelnden Substantiierung. Nun beginnt sie in den Protokollen nach Textstellen zu suchen, welche dieses Argument belegen. Immer wieder springt sie zwischen dem Sachverhalt und den Argumenten hin und her. Während sie weiter nach Argumenten für Unsubstantiiertheit sucht, fallen ihr einige Widersprüche auf, insbesondere zwischen den Protokollen der Gesuchstellerin und denen ihres Bruders. Sie notiert sich diese und nimmt noch den Textbaustein zu »Widersprüchen« hinzu. Während sie schreibt, erklärt sie mir, dass dieser Vergleich hier wichtig sei, weil sie sonst nicht genügend Argumente hätte. Sie glaubt aber, dass andere wohl mit dem Argument der Unsubstantiiertheit zufrieden gewesen wären. Aber sie würde sich damit nicht sicher genug fühlen. Weil sie dem Gesuchsteller nun noch auf schriftlichem Weg das rechtliche Gehör gewähren muss, um diese Widersprüche überhaupt verwenden zu dürfen, legt sie das Dossier weg und wendet sich anderen Aufgaben zu.« (Auszug aus dem Forschungstagebuch)

Das Beispiel zeigt, dass der Fall für Manuela schon zu Beginn des Schreibprozesses klar war: die Aussagen der Frau waren unglaubhaft. Manuela fand jedoch zunächst nicht genügend aktenkundige Tatsachen, die ihr >Gefühl und mit denen sie sich sicher genug fühlte, denn sie wusste, dass sie mit ihren Argumenten sowohl ihren Vorgesetzten, als auch - im Fall einer Beschwerde das BVGer überzeugen musste. Somit war ihr Entscheidungswissen zu Beginn inkomplett. Dies änderte sich mit dem Einbezug des Dossiers des Bruders. Durch den Vergleich der beiden Dossiers fand Manuela Argumente für das Kriterium »Widersprüche«. Es gelang ihr, aktenkundige Tatsachen mit Recht zu verknüpfen: das Entscheidungswissen war komplett. ${ }^{34}$ Zwar wusste Manuela nicht genau, was dieser Frau in Sri Lanka alles widerfahren war. Sie vermutete nämlich, dass die Frau ihre wahren Ausreisegründe nicht erzählt habe. Was ihre Gründe waren und warum die Gesuchstellerin diese nicht erzählt hatte, wusste sie nicht. Sie konnte darüber nur spekulieren. Sie musste es aber auch nicht wissen, denn für einen eindeutigen, legitimen Asylentscheid war ihr Entscheidungswissen genug.

34 Manuela verwendete in ihrem endgültigen Entscheid mehrere Kriterien aus Artikel 7 und Artikel 3 AsylG. Es waren aber vor allem die Widersprüche, die ihr beim Schreiben die Sicherheit gaben, den Entscheid so zu treffen, wie sie es für richtig befand. 


\section{Fazit}

Entscheidungswissen ist stets eindeutiges Wissen. Es erzeugt die begründete »Ja-« oder »Nein-« Beantwortung hierarchisch angelegter Fragen. Dadurch werden Asylgesuche klar einer von vier Rechtskategorien zugeordnet. Entscheidungswissen ist also nicht Wissen um des Wissens willen. Es zielt immer auf ein Verwaltungsprodukt ab: den fertigen Asylentscheid. Das bedeutet, dass Entscheidungswissen nur so komplex und ambivalent sein kann, wie es noch eindeutige »Ja-« oder »Nein-« Antworten zulässt. Gleichzeitig kann es Wissenslücken bestehen lassen, wenn sie für die Entscheidungsfindung irrelevant sind. Dies zeigt das Beispiel der Sachbearbeiterin Manuela. Sie glaubte, dass die Gesuchstellerin andere Gesuchgründe hatte als diejenigen, die sie in den Anhörungen geltend gemacht hatte. Welche das waren, wusste sie nicht, musste sie für den Asylentscheid aber auch gar nicht wissen. Es hätte in der Verantwortung der Gesuchstellerin gelegen, ihr diese Gründe zu schildern. ${ }^{35}$ Eine andere Form von Wissenslücken ergibt sich durch die Wahrscheinlichkeitseinschätzungen, auf denen Artikel 3 und Artikel 7 AsylG beruhen: Bei Artikel 3 geht es um die Einschätzung, wie begründet die Furcht vor zukünftiger Verfolgung ist, und Artikel 7 verlangt nach einer Einschätzung, wie wahrscheinlich das Vorhandensein der Flüchtlingseigenschaft ist. Im Wort »Wahrscheinlichkeit« sind Wissenslücken bereits inhärent, denn Wahrscheinlichkeit kann immer nur auf partiellem Wissen beruhen. So kann die Zukunft nicht mit hundertprozentiger Sicherheit vorausgesagt werden, und sicher wissen, was >wirklich nicht dabei war, kann man auch nicht (vgl. Scheffer 2003: 425). Solche Wissenslücken sind jedoch nur so lange ein praktisches Entscheidungsproblem, wie kein Entscheidungswissen an deren Stelle gesetzt wird.

»In all legal judgments, recognized uncertainty has to be transformed into practical certainty«, schreibt Kelly (2011: 185). Dies gilt auch für das Entscheidungsverfahren im SEM. Die Ausgangslage ist aufgrund der eben genannten Wissenslücken meist eine ungewisse. Durch das Verfahren muss eine »praktische Gewissheit« geschaffen werden; eine Gewissheit, die rechtlich legitimierba-

35 Asylsuchende Personen unterstehen der sogenannten Mitwirkungspflicht nach Artikel 8 AsylG. Diese verlangt, dass Gesuchsteller_innen »ihre Identität offenlegen, vorhandene Reise- und Identitätsausweise und allfällig vorhandene Beweismittel abgeben oder, soweit vorhanden und beschaffbar, sich um ihre Beibringung bemühen « (Schweizerische Flüchtlingshilfe SFH 2009: 79). Des Weiteren müssen die Gesuchstellenden »bei der Befragung detailliert und vollständig [...] begründen, weshalb sie in der Schweiz um Asyl« nachsuchen (ebd.). 
re Entscheide ermöglicht. Diese praktische Gewissheit ist Entscheidungswissen. In meinem Beitrag habe ich aufgezeigt, wie Entscheidungswissen im Verlauf des Verfahrens hergestellt wird. Welche Rolle die verschiedenen Wissensformen dabei spielen, wie sie genau zusammenspielen und sich gegenseitig bedingen, ist fallspezifisch und kann in dieser Komplexität nicht dargestellt werden. Allgemein kann jedoch gesagt werden, dass Entscheidungswissen sich in schriftlicher Form durch die Verknüpfung von Gesetzeskriterien mit aktenkundigen Tatsachen äußert. Diese Verknüpfungen sowie die aktenkundigen Tatsachen selbst müssen jedoch erst über das Verfahren hergestellt werden, wie die Beispiele der Sachbearbeitenden Bernard und Manuela zeigen. Dies geschieht, indem Gesuchstellende auf bestimme Art und Weise befragt werden und indem zusätzliche Abklärungen getätigt werden, wie zum Beispiel Lingua-Analysen, Länderconsultings, Botschaftsabklärungen etc. Die Richtung, in welche aktenkundige Tatsachen generiert werden, sowie deren Verknüpfung mit Gesetzeskriterien werden durch professionelles Wissen geprägt. Entscheidungswissen ist somit mehr als das, was gegen außen sichtbar wird. Es beruht auch auf einer ’gefühlten Sicherheit<.

Am Beispiel von decision-making im SEM habe ich aufzuzeigen versucht, wie im Asylverfahren verschiedene Formen von Wissen produziert und mobilisiert werden. Ich habe dabei Webers Definition des Dienstwissens eine weitere Form hinzugefügt: professionelles Wissen. Weber ging es jedoch nicht >nur darum, »bürokratisches Wissen« zu definieren. Vielmehr argumentierte er, dass bürokratische Verwaltung »Herrschaft kraft Wissen« bedeutet (Weber 2005: 165f.). Dies habe ich auch für das SEM gezeigt. Die Macht des Entscheidungswissens, das $>$ Fälle< überhaupt erst entscheid- und kategorisierbar macht, liegt darin, dass es nicht für alle zugänglich ist. Zwar könnte sich theoretisch Jede_r Länder- und Gesetzeskenntnisse aneignen. Der Einfluss, den Gesuchstellende und andere in das Verfahren involvierte Personen auf die Herstellung von aktenkundigen Tatsachen und deren Verwendung haben, ist jedoch gering. Ferner bleibt professionelles Wissen gegen außen hin stets ganz verborgen.

\section{Literatur}

Alpes, Maybritt Jill/Spire, Alexis (2014): »Dealing with Law in Migration Control: The Powers of Street-level Bureaucrats at French Consulates«, in: Social \& Legal Studies 23(2), S. 261-274.

Anderson, Jessica/Hollaus, Jeannine/Lindsay, Annelisa/Williamson, Collin (2014): »The culture of disbelief. An ethnographic approach to understand- 
ing an under-theorised concept in the UK asylum system. «, in: Working paper series no. 102, Oxford: Refugee Studies Centre.

Bourdieu, Pierre (1976): Entwurf einer Theorie der Praxis auf der ethnologischen Grundlage der kabylischen Gesellschaft, Frankfurt a.M.: Suhrkamp.

Bryman, Alan (2011): Social Research Methods, Oxford: Darin.

Derlien, Hans-Ulrich/Böhme, Doris/Heindl, Markus (2011): Bürokratietheorie: Einführung in eine Theorie der Verwaltung (= Studienskripten zur Soziologie), Wiesbaden: VS Verlag für Sozialwissenschaften.

Fassin, Didier (2013): »The Precarious Truth of Asylum«, in: Public Culture 25(1), S. 39-63.

Fassin, Didier/D'Halluin, Estelle (2005): »The Truth from the Body. Medical Certificates as Ultimate Evidence for Asylum Seekers«, in: American Anthropologist 107(4), S. 597-608.

Glaser, Barney G./Strauss, Anselm L. (1967): The discovery of grounded theory: strategies for qualitative research, Chicago: Aldine.

Good, Anthony (2011): "Witness statement and credibility assessments in the British asylum courts«, in: Livia Holden (Hg.), Cultural Expertise and Litigation, Abingdon, New York: Routledge, S. 94-122.

Hammersley, Martyn/Atkinson, Paul (2007): Ethnography. Principles in practice. Third edition, London/New York: Routledge.

Handelman, Don (1995): »Comments on >Putting Power in the Anthropology of Bureaucray: The Immigration and Naturalization Service at the MexicoUnited States Border` by Josiah McC. Heyman«, in: Current Anthropology 36(2), S. 280-281.

Handelman, Don (2004): Nationalism and the Israeli State. Bureaucratic Logic in Public Events, Oxford/New York: Berg.

Heyman, Josiah McC (2004): »The Anthropology of Power-Wielding Bureaucracies«, in: Human Organization 63(4), S. 487-500.

Heyman, Josiah McC. (2009): »Trust, Privilege, and Discretion in the Governance of US Borderlands with Mexico«, in: Canadian Journal of Law and Society 24(3), S. 367-390.

Hoag, Colin (2011): »Assembling Partial Perspectives: Thoughts on the Anthropology of Bureaucracy«, in: PoLAR: Political and Legal Anthropology Review 34(1), S. 81-94.

Jubany, Olga (2011): »Constructing truths in a culture of disbelief. Understanding asylum screening from within«, in: International Sociology 26(1), S. 74-94.

Kelly, Tobias (2011): »The legalization of human rights and the protection of torture survivors. Asylum, evidence and disbelief«, in: Marie-Bénédicte 
Dembour/Tobias Kelly (Hg.), Are Human Rights for Migrants? Critical Reflections on the Status of Irregular Migrants in Europe and the United States, New York: Routledge, S. 184-200.

Kelly, Tobias (2012): »Sympathy and suspicion: torture, asylum, and humanity«, in: Journal of the Royal Anthropological Institute 18(4), S. 753-768.

Kobelinsky, Carolina (2008): »The Moral Judgement of Asylum Seekers in French Reception Centres«, in: Anthropology News 49(5), S. 5-11.

Lipsky, Michael (2010): Street-Level Bureaucracy. Dilemmas of the Individual in Public Services, New York: Russel Sage Foundation.

Macklin, Audrey (1998): Truth and Consequences: Credibility Determination in the Refugee Context. Conference paper, Ottawa: International Association of Refugee Law Judges.

Scheffer, Thomas (2001): Asylgewährung. Eine ethnographische Analyse des deutschen Asylverfahrens, Stuttgart: Lucius \& Lucius.

Scheffer, Thomas (2003): »Kritik der Urteilskraft. Wie die Asylprüfung Unentscheidbares in Entscheidbares überführt«, in: Jochen Oltmer (Hg.), Migration steuern und verwalten: Deutschland vom späten 19. Jahrhundert bis zur Gegenwart, Göttingen: Vandenhoeck \& Ruprecht, S. 423-458.

Schweizerische Flüchtlingshilfe SFH (Hg.) (2009): Handbuch zum Asyl- und Wegweisungsverfahren, Bern: Haupt.

Souter, James (2011): »A Culture of Disbelief or Denial? Critiquing Refugee Status Determination in the United Kingdom«, in: Oxford Monitor of Forced Migration 1(1), S. 48-59.

Steiner, Pascale (2001): Bourdieu lesen und verstehen (= Arbeitsblätter des Instituts für Ethnologie der Universität Bern, Nummer 19), Bern: Institut für Ethnologie.

Thomas, Robert (2009): »Refugee Roulette: A UK Perspective«, in: Jaya RamjiNogales/Andrew I. Schoenholtz/Philip G. Schrag (Hg.), Refugee Roulette. Disparities in Asylum Adjudication and Proposals for Reform, New York/London: New York University Press, S. 164-186.

Weber, Max (1988): »Parlament und Regierung im neugeordneten Deutschland«, in: Johannes Winckelmann (Hg.), Gesammelte politische Schriften, Tübingen: J.C.B. Mohr (UTB).

Weber, Max (2005): Wirtschaft und Gesellschaft, Nürnberg: Zweitausendeins. 


\section{Internetquellen}

Abkommen über die Rechtsstellung der Flüchtlinge vom 28. Juni 1951: http://www.unhcr.ch/fileadmin/user_upload/unhcr_ch/Mandat/GFK_und_Pr otokoll.pdf vom 24.04.15.

Asylgesetz vom 26. Juni 1998 (Stand 1. Oktober 2015): https://www.admin.ch/ opc/de/classified-compilation/19995092/index.html vom 28.12.15.

Bundesgesetz über die Ausländerinnen und Ausländer (Stand 1. Oktober 2015): https://www.admin.ch/opc/de/classified-compilation/20020232/index.html vom 10.02.16.

https://www.bfm.admin.ch/bfm/de/home/aktuell/news/2015/2015-02-16.html vom 08.04.15.

https://www.bfm.admin.ch/bfm/de/home/asyl/asyl/asylrecht.html vom16.10.15. https://www.bfm.admin.ch/bfm/de/home/asyl/asylverfahren/drei_beispiele/analy sen.html vom 21.04.15.

Staatssekretariat für Migration (SEM): Handbuch Asyl und Rückkehr. Artikel C5 Der Nachweis der Flüchtlingseigenschaft. https://www.bfm.admin.ch/ dam/data/bfm/asyl/verfahren/hb/c/hb-c5-d.pdf vom 24.04.15. 

Die Verwaltung von Asyl als umkämpfte Praxis 



\section{Ausländerbehörden im dynamischen Feld der Migrationssteuerung ${ }^{1}$}

Tobias G. EULE

\section{Einleitung}

Dieses Kapitel beleuchtet die Rolle von Ausländerbehörden, den Behörden, die regulär über den Verbleib von Migrant*innen zu entscheiden haben, im Kontext ihres Verhältnisses zu anderen staatlichen und nichtstaatlichen Akteuren. Der vorliegende Text baut dabei auf bisheriger ethnographischer Forschung zur Rechtsanwendung in Ausländerbehörden auf (vgl. Eule 2014). Die wenigen bestehenden Studien in diesem Feld gehen in der Regel von einem kooperativen und mehr oder weniger guten Verhältnis zwischen staatlichen Kontrollakteuren aus und weisen zivilgesellschaftlichen Akteuren eine kritisch betrachtende oder aktiv intervenierende Rolle zu (vgl. Ellermann 2009; Cyrus und Vogel 2003; Feldman 2012). Dieses recht einfache Bild soll auf Basis von 20 Monaten ethnographischer Feldforschung an fünf Standorten korrigiert werden. Konkret zeigt das Kapitel, dass Beziehungen zwischen staatlichen Akteuren sehr wohl auch konfliktgeladen sind, während Beziehungen zwischen Ausländerbehörden und zivilgesellschaftlichen Organisationen gerade in der Verwaltungskrise 2015 kooperativ und unterstützend waren. Zivilgesellschaftliche Akteure sind daher nicht mehr grundsätzlich als kritische Überwacher, sondern als wohlwollende und in Einzelfällen kritische Begleiter der Arbeit von Ausländerbehörden zu ver-

1 Die Datenerhebung für diesen Beitrag wurde durch Forschungsförderung des Economic and Social Research Council UK (Ref. ES/M003825/1) und des Schweizer Nationalfonds (Ref. 100017_153225) ermöglicht. 
stehen. Diese inzwischen viel dynamischeren Beziehungen sind auf drei Entwicklungen zurückzuführen, die am Ende des Beitrags beleuchtet werden sollen.

Das Kapitel beruht sowohl auf den Ergebnissen der ursprünglichen Feldforschung, als auch auf aktuellen Tätigkeiten des Autors im Rahmen von Beratungstätigkeiten und neuen Feldaufenthalten im Sommer 2015. Neben teilnehmenden Beobachtungen wurden dabei über 70 Interviews mit Experten aus dem Umfeld von Ausländerbehörden geführt - mit Vertretern anderer Ämter, Gerichte, und vielen unterschiedlichen zivilgesellschaftlichen Organisationen. Da der vorliegende Text auf ethnographischer Feldforschung beruht, stellen Vignetten aufbereitete Feldnotizen - einen großen Teil der hier genutzten Daten dar. Aus Platzgründen wird dabei darauf verzichtet, die einzelnen Feldkontexte besonders einzuführen; die vorgestellten Ausschnitte sollen der Argumentation dienen.

Der nächste Abschnitt dient als kurze Einführung in die Aufgaben und $\mathrm{Zu}$ ständigkeiten von Ausländerbehörden sowie den bisherigen Forschungsstand. Danach werden die »kollegialen « Behörden beschrieben, denen in der Regel ein konstant gutes Verhältnis zu Ausländerbehörden zugeschrieben wird: andere Ausländerbehörden, Sozialämter, Polizei und das Bundesamt für Migration und Flüchtlinge (BAMF). Der Schwerpunkt des Kapitels liegt auf der Beschreibung des Verhältnisses von Ausländerbehörden und Institutionen, denen die Literatur ein eher kritisches Verhältnis zuspricht, vornehmlich Akteuren der Zivilgesellschaft. Deren in Wirklichkeit komplexe und teilweise unterstützende Verbindung zu Ausländerbehörden wird in einem dritten Teil beschrieben, bevor schließlich drei Ursachen benannt werden, die einen Wandel im Feld der Migrationsverwaltung hervorgebracht haben: Veränderungen von Recht, der Ausrichtung und Zuständigkeit von Akteuren.

\section{Was sind Ausländerbehörden?}

Während im konkreten Asylverfahren die Prüfung über die Plausibilität und Glaubwürdigkeit eines einzelnen Antrags Spezialisten mit zumindest einer gewissen zentralisierten Ausbildung obliegt, werden wie in vielen anderen europäischen Ländern alle anderen aufenthaltsrechtlichen Belange von kommunalen Ausländerbehörden behandelt. Dies umfasst im Asylkontext sowohl die Ausstellung und Verlängerung der Gestattung des Aufenthalts zur Durchführung des Asylverfahrens (nach §55 Abs. 1 AsylVfG), die Ausstellung und Verlängerung von Aufenthaltserlaubnissen »aus völkerrechtlichen, humanitären oder politischen Gründen« (nach Kapitel 2, Abschnitt 5 des Aufenthaltsgesetzes), als auch die Ausweisung und Durchführung aufenthaltsbeendender Maßnahmen (nach 
dem fünften Kapitel des Aufenthaltsgesetzes) sowie in den meisten Fällen die Entscheidung über eine mögliche Einbürgerung der Betroffenen. Während die Mitarbeitenden des Bundesamts für Migration und Flüchtlinge also im Asylprozess an sich eine erhebliche Entscheidungsmacht ausüben, sind alle daraus resultierenden Aufenthaltstitel und die Frage nach dem möglichen Weiterverbleib oder der Ausreise Aufgabe der Ausländerbehörden.

Da Ausländerbehörden im Gegensatz zu den Außenstellen des BAMF in Trägerschaft der Kreise und kreisfreien Städte liegen, unterscheiden sich erstere ganz erheblich in Größe, Ausstattung und Professionalisierung. Während in kleinen, ländlichen Kreisen mit geringem Migrant*innenanteil oft Einzelpersonen »die« Ausländerbehörde darstellen, haben Ausländerbehörden in Hamburg oder Berlin durchaus mehrere 100 Mitarbeitende. Auch wenn sie die im Großen und Ganzen gleiche Rechtsgrundlage (das Aufenthaltsgesetz und zugehörige Verfahrensvorgaben sowie zahlreiche andere nationale und europäische Rechtsquellen) haben, können der Arbeitsalltag und die Anwendungspraxis in Ausländerbehörden äußerst unterschiedlich sein. Hierzu gibt es bisher relativ wenig wissenschaftliche Daten (vgl. Boswell und Geddes 2011; Feldman 2012; Morris 2002). Eine von mir durchgeführte ethnographische Untersuchung der Arbeitspraxis von vier unterschiedlichen Behörden (Eule 2014) bestätigt aber, dass gerade in der Entscheidungsfindung und Sachverhaltsklärung sowohl innerhalb als auch zwischen Ausländerbehörden immense Unterschiede bestehen. Diese Unterschiedlichkeit scheint dabei mit der zunehmenden Prekarität des Aufenthaltsstatus zu steigen: je unsicherer der Verbleib in Deutschland, desto unterschiedlicher die Verwaltungspraxen in den beforschten Ämtern.

Ausländerbehörden sind damit also nicht nur wirkungsmächtige Akteure in der Verwaltung von Asyl, sondern auch in vielen Fällen unberechenbar. Dies liegt an vielen Faktoren, etwa der hohen Komplexität und ständigen Veränderung des geltenden Rechts oder der relativ geringen Vergütung und schlechten Ausbildung der Mitarbeitenden. In keiner der vier Behörden gab es zum Zeitpunkt der Feldforschung ein strukturiertes Einarbeitungs- oder Fortbildungskonzept, so dass neue Sachbearbeiter*innen einerseits ihrer Eigeninitiative überlassen waren, andererseits aber durch das Zuschauen und Abgucken etablierter behördlicher Praktiken lernten. Im Ergebnis lag in allen beforschten Ämtern ein hoher Grad an Informalität vor, der zwar einerseits Mitarbeitenden Hilfe und Leitung anbot, andererseits aber Rechtsanwendung stärker als erwartet (oder manchem lieb sein mag) von mündlichen Überlieferungen und nicht Textkenntnis abhängig machte. Sachbearbeiter*innen entschieden dabei nicht gänzlich willkürlich, sondern in eigener (und oftmals kollektiver) Interpretation und Anwendung tradierter Geschichten über das Recht (vgl. Eule 2014). Neben internen 
Faktoren spielen dabei auch diverse externe Faktoren eine große Rolle, auf die in diesem Beitrag der Schwerpunkt gelegt werden soll.

Das Gros der Forschungsliteratur zu Migration hat bisher über die Frage der praktischen Migrationsverwaltung einen weiten Bogen gemacht (vgl. Boswell und Geddes 2011; Morris 2002). Mit der Abwendung vom Nachzeichnen breiter nationaler (und stereotyper) Unterschiede zwischen Migrationspolitiken und der Hinwendung zu kleinteiligeren, weniger normativen und komplexeren Steuerungsstrategien (vgl. Geiger und Pécoud 2012; Geiger und Pécoud 2010) in den letzten zehn Jahren sind auch einige Studien über Migrationsverwaltung $\gg$ in action« erschienen (z.B. Ellermann 2009; Cyrus und Vogel 2003; Eule 2014; CôtéBoucher, Infantino und Salter 2014; eine frühe und sehr mikrosoziologische Ausnahme ist Scheffer 1995; Scheffer 2001). Diese Studien unterscheiden sich in Fokus und Qualität, kommen aber oft zu dem Ergebnis, dass zwischen Migrationsverwaltung und Zivilgesellschaft ein kritisches, grundsätzlich eher antagonistisches Verhältnis besteht (v.a. Ellermann 2009; Borgards 2006; Anderson 2011). Studien wie Ellermans belegen, dass lokale zivile Akteure in der Regel gegen Ausländerbehörden mobilisieren, selbst wenn die öffentliche Meinung im Staat für schärfere Migrationskontrollen ist. Diese Annahme findet sich auch in eher theoretischen oder normativen Texten (Castles 2004a, 2004b; Hollifield 2004) und in Literatur, die sich auf den lokalen Kontext von Migration bezieht (Buckel 2011).

In der Tat findet sich diese Perspektive der kritischen zivilen Akteure auch in der Praxis wieder. Größere zivilgesellschaftliche Organisationen wie Pro Asyl agieren als wichtiges und in Teilen wirkmächtiges Kontrollorgan zu rechtspolitischen Debatten auf nationaler, aber auch lokaler Ebene. Die zwei großen Fachzeitschriften des Ausländerrechts, der Informationsbrief Ausländerrecht und die Zeitschrift für Ausländerrecht und Ausländerpolitik mit den Anwaltsnachrichten Ausländer- und Asylrecht zeigen regelmäßig auch »worst practice«-Beispiele der Migrationsverwaltung auf. Auf konzeptioneller wie textueller Ebene scheint ein grundsätzlich kritisches Verhältnis zwischen Ausländerbehörden und Zivilgesellschaft also plausibel.

Dies deckt sich in Teilen mit den Erfahrungen zur praktischen Kooperation, die im Laufe der Datenerhebung erhoben werden konnten. Aus allen Perspektiven gibt es in den Interviews Anekdoten über das historisch schlechte Verhältnis der beteiligten Akteure, über »Presseschlachten«, direkte Konfrontationen und ein grundsätzlich misstrauisches Miteinander. Gleichzeitig hatten diese Berichte immer auch einen historischen Kontext - sie stellten Erlebnisse der Vergangenheit und nicht die aktuellen Erfahrungen dar und wurden in der Regel verwendet, um auf ein inzwischen anderes Verhältnis hinzuweisen. Diesem gilt es im vor- 
liegenden Beitrag nachzuspüren. Es soll dabei aufgezeigt werden, dass verschiedene Akteure der Migrationssteuerung zwar sehr wohl auf Grund ihrer Aufgabe zusammenhängen (vgl. Feldman 2012), aber nicht notwendigerweise besonders gute Verhältnisse miteinander haben müssen. Im Gegensatz dazu ist das Miteinander zwischen Ausländerbehörden und lokalen zivilgesellschaftlichen Organisationen häufig viel weniger von Kritik als von Kooperation und gegenseitiger Unterstützung gezeichnet.

\section{Akteure der Migrationssteuerung}

Neben der Ausländerbehörde sind fünf weitere staatliche Organisationen mit der Verwaltung von Migration beschäftigt. Einerseits liegt es im Aufgabenbereich der Polizeien der Länder und des Bundes, unerlaubte Einreise, Aufenthalt und Arbeitsaufnahme zu ermitteln. Dies ist Teil des regulären Streifendienstes, es gibt aber auch in vielen Bundesländern spezielle Einheiten, in denen Daten zu diesen Straftatbeständen erfasst und ausgearbeitet werden. Darüber hinaus ist die Polizei bei verschiedenen ausländerbehördlichen Aktivitäten, gerade etwa begleiteten Abschiebungen, in Amtshilfe aktiv. Gerichte spielen in Widerspruchsfällen und bei Haftanträgen wichtige Rollen und haben darüber hinaus auch in klassisch kodifizierten »civil law«-Systemen oft eine unmittelbar rechtssetzende Funktion. Auf sie wird in anderen Kapiteln dieses Sammelbandes genauer eingegangen, weswegen sie hier weniger betrachtet werden sollen. Das Bundesamt für Migration und Flüchtlinge (BAMF) entscheidet über Asylfälle, ist aber auch in Fragen der sprachlichen Integration (über die Sprach- und Integrationskurse) sowie teilweise bei der Koordination von »freiwilliger Rückkehr« mit Rückkehrhilfen der Internationalen Organisation für Migration (IOM) aktiv. Verschiedene lokale Behörden spielen in der Verwaltung des Alltags eine große Rolle. Hierzu gehören einerseits die Arbeitsämter, die über die Anerkennung von Qualifikationen, die Einreihung in Erwerbsprogramme sowie die Beschäftigung in zustimmungspflichtigen Berufen zuständig sind. Eine zweite, noch unmittelbar wichtigere Behörde ist das Sozialamt, das in der Regel eine spezielle Stelle für Asylfragen - oft Asylbewerberleistungsstelle - hat. Hier werden Unterkunft und Versorgung der Asylbewerber*innen und anerkannten Flüchtlinge organisiert. Schließlich gibt es in manchen Bundesländern Stellen des Landes (Landesausländerbehörde, zentrale Ausländerbehörde, Landesamt für Migration oder Landesamt für Flüchtlingsfragen genannt und jeweils den Abteilungen für Ausländerrecht des jeweiligen Ministeriums für Inneres zugeordnet), die einerseits Erstaufnahmeunterkünfte für neue Asylbewerber*innen unterhalten, andererseits 
in Amtshilfe Aufgaben im Rückführungsbereich - vor allem Passbeschaffung und Massentransporte - übernehmen.

All diesen Stellen ist gemein, dass sie staatlich finanziert, beauftragt und von Beamten oder Angestellten des Staats besetzt sind. Als Behörden des Bundes, der Länder oder der Kommunen haben sie natürlich unterschiedliche Dienstherren und sind mit sehr unterschiedlich spezialisiertem und geschultem Personal besetzt, dennoch ist die staatliche Anstellung und damit Repräsentanzrolle gegenüber Migrant*innen ein entscheidender gemeinsamer Faktor. So werden Vertreter*innen von Ausländerbehörden, die bei anderen Stellen vorsprechen, sowohl im Sozialamt als auch bei der Polizei als »Kollegen« wahrgenommen dies steht im klaren Widerspruch zu den zivilgesellschaftlichen Organisationen, die im folgenden Teil besprochen werden sollen. Zum Verwaltungsgericht besteht hier eine größere Distanz, allerdings eine eindeutig größere Nähe als zwischen Gericht und einzelnen Betroffenen.

Es ist zu erwarten, dass auf Grund dieser strukturellen Nähe auch eine gewisse enge Zusammenarbeit zwischen den genannten Behörden und den Ausländerbehörden existiert. Dies stimmt in vielen Fällen auch, ist aber gleichzeitig fast immer zu qualifizieren. Nur weil zwei Behörden »den Staat« repräsentieren und oft ähnliche Aufgaben übernehmen, heißt es nicht, dass sie besonders gut kooperieren oder grundsätzlich miteinander auskommen. Gute Verhältnisse sind in der Regel nicht Ausdruck einer strukturellen Logik, sondern interpersonaler Faktoren und bedürfen eines regen und informellen Austauschs.

Bei einem Treffen regionaler Ausländerbehörden sind auch Vertreter*innen der Polizei geladen und ein Vertreter der »Fachstelle illegaler Aufenthalt und Schwarzarbeit« $\left(\right.$ FIAS) $*^{2}$ der Landespolizei ist angefragt worden, um einen Vortrag über die aufenthaltsrechtlichen Maßnahmen zu halten, die sich aus dem Schengener Visa-Kodex ergeben. Hiernach sind Visa, deren Inhaber erkennbar einem anderen Zweck nachgehen als gedacht (z.B. Touristen, die arbeiten), sofort ungültig zu erklären - die betroffene Person verliert also sofort ihre Aufenthaltsberechtigung. In der entsprechenden Region (immerhin mit ca. 3 Millionen Einwohnern) hätten Ausländerbehörden in den fünf Jahren der Geltung des Visa-Kodex bisher keinen einzigen solchen Fall bearbeitet - dies scheint den FIASVertreter ungemein zu stören. Beim gemeinsamen Mittagessen erklärt er mir seinen Frust - die Ausländerbehörden würden ihren rechtlichen Auftrag nur höchst selektiv erfüllen, dabei seien die Visa-Annullierungen ohne Ermessen auszuführen und auch noch ziemlich einfach zu machen. Die Vertreter*innen der Ausländerbehörden sehen die Sache etwas

2 Dies ist ein anonymisiertes Akronym und soll keinen Rückschluss auf das Bundesland geben. 
anders. Schon während des Vortrags wird klar, dass kaum einer der Vertreter*innen besonders aufmerksam ist, und auch bei der anschließenden Diskussion hält sich das Interesse in Grenzen. Als ich während einer Kaffeepause mit verschiedenen Teilnehmenden das Thema anspreche, winken diese nur ab. Man könne sich nicht mit jeder kleinsten EUBestimmung auseinandersetzen, das schaffe nur zusätzliche Arbeit und würde nicht helfen, Fälle abzuarbeiten. Außerdem solle doch die Polizei den Touristen hinterherschnüffeln, wenn die das so wichtig fänden.

Etwas anders ist die Lage im Spätsommer 2015, als klar wird, dass die lokale Polizei Geflüchtete nicht nur nicht an ihrer Weiterreise in Richtung Skandinavien hindert, sondern diese aktiv begleitet und etwa Familien hilft, vom Busbahnhof zum Bahnhof zu finden, ohne sie nach (natürlich nicht vorhandenen) Aufenthaltspapieren zu fragen. Eine der im Fall des Visa-Kodex noch so entspannten Ausländerbehördenvertreter*innen ist hier sehr aufgebracht und bezichtigt die Polizei der Beihilfe zum illegalen Aufenthalt - kurz, des so heiß diskutierten Menschenschmuggels. Statt Verständnis für dieses Verhalten zu zeigen, findet sie es verantwortungslos und erklärt, dass man stattdessen ja gleich das Dubliner Übereinkommen aufkünden könne.

Bei der Definition und Erfüllung ihres gesetzlichen Auftrags sind sich Polizei und Ausländerbehörde also nicht immer einig. Wie die obigen Beispiele zeigen, kann dies durchaus in unterschiedliche Richtungen gehen. Während im ersten Beispiel die Polizei die Einhaltung einer supranationalen Richtlinie für immens wichtig erklärt, ist es im zweiten Fall die Ausländerbehörde, die der Polizei selektives Verhalten und Missachten von EU-Recht vorwirft. Trotz des grundsätzlich kollegialen Verhältnisses wäre es also viel zu simpel, davon auszugehen, dass diese zwei Behörden stets am gleichen Strang ziehen und eine rechtliche oder praktische Position vertreten. Diese Meinungsverschiedenheiten sind dabei nicht trivial. Während die Zahl der mit Touristenvisum arbeitenden Personen schwer zu schätzen ist, geht man offiziell davon aus, dass 2015 mindestens 60000 Geflüchtete Deutschland gen Skandinavien wieder verlassen haben - diese Zahl beruht auf Zählungen der Polizei, die den Prozess nicht nur nicht verhindert, sondern aktiv begleitet hat.

Während das Verhältnis zur Polizei noch relativ gut ist, scheint die Beziehung zum BAMF fast traditionell schlecht. Fast alle der Ausländerbehörden, mit denen ich im Rahmen der Feldforschung in Berührung gekommen bin, beklagten sich über schlampige Arbeit, schlechte Erreichbarkeit und Unberechenbarkeit der »Kollegen« des BAMF. Dies wurde im Sommer 2015 durch die rapide ansteigenden Fallzahlen und zu spät genehmigten Personalaufstockungen noch spürbarer. In Transitstadt, meinem Feldaufenthalt 2015, wurde fast jeder zugewiesene Geflüchtete mit einer vom BAMF falsch ausgestellten Aufenthaltsge- 
stattung (dem Aufenthaltsstatus während des Asylverfahrens) zur Ausländerbehörde geschickt, was für jede betroffene Person eine zusätzliche Vorsprache und für die Mitarbeitenden der Ausländerbehörde natürlich erheblichen Mehraufwand bedeutete. Versuche, das lokale BAMF-Büro zu erreichen, scheiterten unter anderem daran, dass das Büro überhaupt nicht erreichbar war und auch auf E-Mails nicht antwortete. Als eine über das Innenministerium geleitete Anfrage mit der Aussage beantwortet wurde, man sei leider überarbeitet und könne den Fehler nicht vermeiden, schienen nicht wenige Mitarbeitende der Ausländerbehörde geneigt, mit Fackeln und Mistgabeln vor das BAMF zu ziehen. Man habe Verständnis für die schwierige Situation und sei geduldig, was Registrierungs-, Entscheidungs- oder allgemeine Kommunikationszeiten angehe. Aber mit systematischen Fehlern den ebenso überarbeiteten Ausländerbehörden zusätzliche Arbeit zu verschaffen, wurde als großer und nicht leicht heilbarer Affront wahrgenommen. Die als Retaliation geplanten Trotzreaktionen konnten zwar durch die Beschwichtigung der Behördenleitung verhindert werden, aber dennoch war im Folgenden häufiger zu beobachten, dass Anfragen vom BAMF ganz nach unten auf den Rückstandsstapel gelegt wurden.

Das Verhältnis zu Mitarbeitenden der lokalen Sozialämter war hier deutlich besser. Dies mag überraschen, da von allen genannten staatlichen Akteuren der Migrationssteuerung die Sozialämter am ehesten eine gegensätzliche Position einnehmen könnten. Solche Konflikte sind offenbar früher durchaus vorgekommen; gleichzeitig waren sich alle Ausländerbehörden darin einig, dass das Verhältnis zum Sozialamt nie so schwierig gewesen sei wie zu Akteuren der Zivilgesellschaft. Obwohl man deutlich unterschiedliche Aufgaben zu erfüllen sah, gab es aus Sicht der beteiligten Behörden keinen Grund, nicht dennoch gut miteinander auszukommen. Dies lag insbesondere daran, dass viele Mitarbeitende von Ausländerbehörden und Sozialämtern sich kannten, da sie vom selben Dienstherren (dem Landkreis oder der kreisfreien Stadt) beschäftigt wurden und sich ihre Wege so häufig gekreuzt haben. Das gute persönliche Verhältnis konnte hier die oftmals unterschiedliche rechtliche Perspektive zu Flucht und humanitärer Migration überdecken. Im Ergebnis war das Vertrauensverhältnis zu den Sozialämtern sogar stärker als das zum den Ausländerbehörden eigentlich viel näher stehenden BAMF.

In Transitstadt wurde dies dadurch besonders deutlich, dass die Asylbewerberleistungsstelle den Ausländerbehörden bei der Registrierung der zugewiesenen Asylsuchenden unter die Arme griff, in dem man die relevanten Zuweisungspapiere und den neuen Ankunftsnachweis BÜMA (ein 2015 eingeführter Status, der vorläufig anstelle einer - ebenso vorläufigen - Aufenthaltsgestattung gilt) der Neuankommenden kopierte und damit den Geflüchteten - und der Aus- 
länderbehörde - immerhin eine Vorsprache ersparen konnte. Diese Arbeitserleichterung wurde »auf kurzem Dienstweg« von zwei befreundeten Sachbearbeiter*innen organisiert, die ihre jeweiligen Vorgesetzten umgingen und vor vollendete Tatsachen stellten. Auch hier ist die persönliche und informelle Zusammenarbeit wieder symptomatisch für die Arbeit der Ausländerbehörden allgemein - viele Schwierigkeiten im Arbeitsprozess wurden durch individuelle Vorstöße geklärt.

Der obige Abschnitt zeigt also, dass die unterschiedlichen Akteure der Migrationssteuerung keinesfalls als einig oder gar koordiniert agierend verstanden werden können. Wie die Beispiele des Visumsmissbrauchs oder der irregulären Durchreise zeigen, sind Situationen, in denen Migrant*innen von laxer Rechtsdurchsetzung profitieren können, genauso Produkt von Entscheidungen spezifischer lokaler Akteure wie es etwa die Entscheidungen über die Durchführung von Abschiebungen sind. Das Verhältnis der Akteure kann durchaus von Misstrauen geprägt sein (wie häufig zwischen BAMF und Ausländerbehörden zu beobachten); ähnliches Misstrauen also, wie man zwischen Ausländerbehörden und Migrant*innen oder zivilgesellschaftlichen Akteuren erwarten könnte. Der folgende Abschnitt soll letztere Organisationen genauer ins Bild nehmen und beleuchten, in wie weit hier Kooperationen oder Friktionen vorherrschen.

\section{Akteure der Zivilgesellschaft}

Transitstadt, Frühsommer 2015. Seit Monaten steigen die Zahlen der Geflüchteten, die dringlich - untergebracht und - weniger dringlich - in den kommunalen Ausländerbehörden registriert werden müssen. Seit Monaten werden dabei den städtischen Behörden Asylbewerberleistungsstelle wie Ausländerbehörde - falsche Aufnahmezahlen aus den Erstaufnahmeeinrichtungen übermittelt, so dass erst jeden Dienstag, danach fast täglich Unterbringungen und Registrierungen improvisiert werden müssen. Um im regulären Arbeitsprozess zu bleiben, muss erst jeder Neuangekommene von der Ausländerbehörde registriert werden, um dann zu Fuß zur Asylbewerberleistungsstelle gehen. Der Weg beträgt kaum 5 Minuten, ist aber relativ schwer zu erklären, wenn man keine gemeinsame Kommunikationssprache hat. Dies jeweils individuell zu tun, kostet Zeit; Zeit, die die Mitarbeitenden der Ausländerbehörde nicht haben. Im Gegensatz zur Leistungsstelle, wo man die Zahl der Beschäftigten seit Anfang 2015 vervierfacht hat, sind hier keine zusätzlichen Stellen geschaffen worden, die Behörde ist im Gegenteil eher unterbesetzt, die Mitarbeitenden in der Situation, reguläre Termine und ungeplant im wahrsten Sinne des Wortes abgeladene Geflüchtete parallel abzuarbeiten. Von Tag zu Tag ist spürbar, wie die Sachbearbeiter*innen hektischer, nervöser, abgearbeiteter werden. Vor wie hinter den Tresen 
herrschen zunehmend chaotische Zustände - wilde Dokumentenstapel türmen sich auf und stürzen ein, Pässe gehen verloren, zu viele Menschen mit viel Gepäck drängen sich in den engen Wartebereichen, die regulären Migrant*innen beschweren sich über Wartezeiten, der Brandschutzbeauftragte über die Versperrung von Fluchtwegen.

Wie an vielen Orten in Deutschland ist das Chaos in der transitstädter Ausländerbehörde ein lösbares logistisches Problem, das durch Fehleinschätzungen und Fehlkalkulation ausgelöst wurde. BAMF und in Grenzen auch die Aufnahmestelle des Bundeslandes senden falsche Prognosen auf Grund falscher Zahlen (etwas, das erst im August aufgelöst wird), die Kommunen schätzen die Lage völlig falsch ein und begreifen erst viel zu spät, dass Geflüchtete auch Migrant*innen sind und damit auch die Ausländerbehörden zusätzlich belasten. In der Behörde selbst führen die ständige Unruhe und hohe Belastung dazu, dass bestehende, aber nicht mehr sinnvolle Prozesse nicht hinterfragt, sondern strikt befolgt werden, weil dieses immerhin gewisse Sicherheit vermittelt. Ähnlich dem von Janis beschriebenen Gruppenverhalten werden so aus eigentlich dynamischen Deliberationsprozessen sich verselbstständigende Entwicklungen, die oft idealere Lösungen ignorieren (Janis 1972). In Transitstadt befand sich die Ausländerbehörde im Sommer 2015 an einem Punkt, wo weder Vorgesetzte noch Sachbearbeitende Zeit oder Nerven für klärende Gespräche hatten und sich so Ineffizienz durchsetzte.

Der entscheidende Lösungsimpuls kam von außen. Unterstützt von verschiedenen größeren Organisationen begann ein kleiner Verein in christlicher Trägerschaft, den Fluss durch den lokalen Bürokratiewust zu organisieren. Das Gepäck der Geflüchteten wurde zwischengelagert, die Menschen in Gruppen von Behörde zu Behörde geführt, Sprachmittler*innen organisiert, um auf den Fluren zu vermitteln, erklären und beruhigen. Bemerkenswert war dabei, dass sich die Hilfsbereitschaft ausdrücklich auch auf die Behördenmitarbeitenden bezog: Die Koordinator*innen sprachen regelmäßig vor, um sich für den Einsatz der Ausländerbehörde zu bedanken, um ihr Verständnis und ihr Mitgefühl auszudrücken und zu erklären, was sie jetzt versuchten, um die Situation für alle Beteiligten zu verbessern. Neben praktischen Aspekten vor Ort gehörte dazu, dass der Verein sich mit bissigen Beschwerdemails an BAMF und Landesaufnahmestelle wandte und die Auswirkungen der chaotischen Arbeit dort anprangerte. Ein zivilgesellschaftlicher Verein, der sich bei anderen Akteuren der Migrationssteuerung für die Ausländerbehörde stark macht - eine überraschende Situation.

Der hier beschriebene Fall ist extrem, aber dennoch aussagekräftig für das veränderte Verhältnis zwischen zivilgesellschaftlichen Akteuren und der Ausländerbehörde. Das in der Literatur erwähnte klassische Beispiel - Zivilgesell- 
schaft mobilisiert gegen Ausländerbehörden - konnte in keinem der untersuchten Standorte zwischen 2009 und 2015 in Reinform gefunden werden. Stattdessen überwogen Formen der lockeren bis festen Kooperation, des Austauschs und gegenseitigen Verständnisses. Dies soll in keinem Fall heißen, dass zivilgesellschaftliche Organisationen sich notwendigerweise zu Komplizen des Aufenthaltsrechts machen, oder dass es keine unterschiedlichen Rechtsmeinungen über Einzelfälle mehr gibt. Stattdessen ist bei der Beschreibung des Verhältnisses von Ausländerbehörden und Zivilgesellschaft von einer anderen Grundfigur auszugehen. Nicht grundsätzlich kritische Überwachung und Gegenpol, sondern eine wohlwollende und in Einzelfällen kritische Begleitung beschreibt das Verhältnis in den fünf beforschten Städten.

Sowohl Ausländerbehörden als auch zivilgesellschaftliche Organisationen scheinen stark über gewachsene und überwiegend informelle Strukturen und Prozesse zu funktionieren (vgl. Eule 2014, Kapitel 4). Erfahrene Mitarbeitende als Art institutionelles Gedächtnis sind hier besonders wichtig. Ähnliches gilt mit Abstrichen - für Anwälte, wo in vielen Städten alteingesessene »Platzhirsche« den Markt beherrschen. Mit Ausnahme von einzelnen Vertreter*innen einer sehr politisch aktiven NGO, die ich zu Beginn meiner Feldforschung interviewte, waren sich aber alle Gesprächspartner*innen einig, dass sich das Verhältnis zwischen Ausländerbehörden und nichtstaatlichen Akteuren - insbesondere aber lokalen zivilgesellschaftlichen Organisationen - grundlegend geändert habe. Die bisher in der Literatur beschriebenen Eindrücke scheinen also zum Zeitpunkt der jeweiligen Erhebung durchaus plausibel gewesen zu sein (und Konflikte zwischen den Parteien gibt es ja nach wie vor), das grundsätzliche Verhältnis scheint sich aber verändert zu haben. Drei Aspekte erscheinen besonders relevant:

Vertreter*innen von Ausländerbehörden und Zivilgesellschaft stehen in einem persönlichen, informellen und vertrauensvollen Austausch. So banal es klingen mag: »wir reden jetzt direkt miteinander« wird fast durchgehend als wichtigster Faktor für die Veränderung der Beziehungen genannt. Statt schriftlich oder über Mittler (Presse, Integrationsabteilungen, Parteien) zu kommunizieren, sei es jetzt möglich, Fragen direkt miteinander zu besprechen. Zivilgesellschaftliche Organisationen scheinen nun besser über die Organisation der Ausländerbehörden informiert und in der Lage zu sein, direkte Ansprechpartner*innen zu finden, um Einzelfälle zu besprechen. Im Umkehrschluss haben Ausländerbehörden begriffen, dass ein - eventuell lästiger - direkter Austausch sinnvoller ist als ein kritischer Pressebericht. Dieses Miteinander hat in der Regel unterschiedliche Quellen. Häufig werden gemeinsame Teilnahmen an Ar- 
beitskreisen erwähnt, manchmal das Engagement einzelner »Türöffner«, manchmal schlicht und ergreifend »sich verändernde Zeiten«.

Neben der veränderten Form der Kommunikation scheint sich auch das Bild des anderen verändert zu haben. Dies zeigen die Experteninterviews. Nicht mehr »rassistische Beamtenfossile« gegen »naive Sozialromantiker« bestimmen die gegenseitigen Charakterisierungen. In der gegenseitigen Einschätzung sind die jeweiligen Organisationen professioneller und pragmatischer geworden. Dieser Eindruck deckt sich zumindest teilweise mit den erhobenen Daten aus den teilnehmenden Beobachtungen, wobei natürlich die Einschätzung hier die viel relevantere, weil Handlung anleitende Variable ist. Nicht nur kann man miteinander reden, ein Austausch kann für beide Parteien zielführend und vorteilhaft sein. Entscheidend sind hier die Erfahrungen, dass schwierige Einzelfälle so gemeinsam geklärt werden konnten. In der Folge werfen sich die beteiligten Akteure nicht mehr vor, nur von starren politischen Positionen auszugehen, sondern erkennen an, dass man in der Sache, nicht aber im Prinzip argumentiere. In den Interviews loben Vertreter*innen von großen NGOs regelmäßig die Ausländerbehörden für ihre Bereitschaft, auch mal großzügige Prognoseentscheidungen zu fällen. Andersherum betonen Mitarbeitende der Ausländerbehörden, dass zivilgesellschaftliche Akteure auch verstehen könnten, wenn rechtlich kein Spielraum gegeben sei, um einen Fall positiv zu entscheiden, und dann auch die Versagung von Aufenthalten und Ausweisungen akzeptieren würden.

Dieses andere Bild und dieser direkte Austausch finden sich in Ansätzen auch in unterschiedlichen Kooperationsstrukturen wieder. Hier sind unterschiedliche Arbeitskreise, vor allem aber einerseits Migrationsberatungen im behördlichen Alltag und andererseits sogenannte lokale Härtefallkommissionen zu betonen. Diese haben theoretisch das Potential, behördliche und zivilgesellschaftliche Ansätze miteinander zu verzahnen und verfestigen. Beratungsangebote innerhalb von Ausländerbehörden können zum Beispiel sich unmittelbar an Betroffene wenden und ohne großen Zeit- oder Informationsverlust schwierige Fälle - in anderen Sprachen als Deutsch oder Englisch - begleiten, erklären und kontextualisieren. Über Härtefallkommissionen können vertrackte Fälle in einem klaren Rahmen diskutiert werden und so einen sachlichen, ergebnisorientierten Prozess vorgeben. Meine Daten ergeben zur Wirkung dieser Strukturen ein (noch) eher negatives Bild (vgl. Eule 2014): In vielen Fällen scheint der einzige Vorteil der geschaffenen formellen Kooperationen zu sein, dass informeller Austausch gestärkt wird, während die offiziellen Ziele auf Grund schlechter Verzahnung und unterschiedlicher Interessen noch nicht erreicht werden. Nichtsdestotrotz ist schon der alleinige Versuch, Ausländerbehörde und Zivilgesellschaft in 
eine feste Zusammenarbeitsform zu bringen, bemerkenswert und - zumindest in der Theorie - vielversprechend.

Ich besuche eine Regionalkonferenz der Migrationsberater*innen einer großen zivilgesellschaftlichen Organisation. Ziel ist es, sich über neue rechtliche Veränderungen, besonders schwierige Fälle und ein gemeinsames Vorgehen im Kontext der sich verändernden Flüchtlingszahlen auszutauschen. Im Vorfeld setzen sich verschiedene Vertreter*innen für einen Vormittag mit mir zusammen, um meine Eindrücke des Miteinanders zwischen Ausländerbehörden und zivilgesellschaftlichen Akteuren zu diskutieren. Diese Form von Triangulation meiner Ergebnisse ist häufig besonders nützlich, da hier nicht offene Fragen, sondern vorläufige Analyseergebnisse besprochen werden und die (teils vorher interviewten, teils neuen) Informant*innen so darauf reagieren können.

Meinen Einschätzungen stimmen sie grundsätzlich zu und führen diese weiter aus. Früher habe man gedacht, dass die Ausländerbehörde »der Feind« sei, den es mit allen Mitteln zu bekämpfen gelte. Jetzt könne man viel besser miteinander umgehen, weil man begriffen habe, dass dort nicht nur Idioten und Rassisten säßen. Das Miteinander sei zwar von Behörde zu Behörde unterschiedlich; die Institutionalisierung noch sehr uneben und man sei in vielen Fällen nach wie vor unterschiedlicher Ansicht. Grundsätzlich sei aber im Vergleich zu den Neunzigerjahren das Verhältnis grundsätzlich anders, viel besser, offener, vertrauter. Erfahrene Berater*innen teilen ihre Schauergeschichten, während jüngere Kolleg*innen ihre Erleichterung darüber teilen, dies nicht mehr mitmachen zu müssen.

Die Zunahme ausreisepflichtiger Migrant*innen wird das Verhältnis von zivilgesellschaftlichen Akteuren und Ausländerbehörden stark belasten und wahrscheinlich in vielen Fällen wieder offene Konflikte hervorrufen. Nichtsdestotrotz lohnt es sich, auf die grundlegend unterschiedliche Ausgangssituation gut fünfundzwanzig Jahre früher hinzuweisen. Im Folgenden sollen drei parallel verlaufende Entwicklungen beschrieben werden, die diese Veränderung mitbeeinflusst haben. Erstens hat sich die rechtliche Basis in Deutschland insbesondere in den letzten fünfzehn Jahren grundlegend verändert. Zweitens hat der Ausbau der Integrationsförderung dazu geführt, dass zivilgesellschaftliche Akteure besser ausgestattet, aber auch staatsnaher geworden sind. Drittens haben sich Ausländerbehörden in ihrer Struktur und Ausrichtung stark verändert. 


\section{Ursachen für ein dynamischeres Miteinander}

\subsection{Die Liberalisierung des Migrationsrechts in Deutschland}

Seitdem die sogenannte Flüchtlingskrise eine starke negative öffentliche Reaktion und internen Koalitionsstreit ausgelöst hat, wurde insbesondere das Asylrecht mehrfach verschärft. In diesem Kontext von einer Liberalisierung des Migrationsrechts zu sprechen, mag arg naiv klingen (und die weitere Entwicklung ist nicht abschätzbar), aber der über die letzten fünfzehn Jahre gewachsene Trend ist dennoch nicht zu ignorieren, weil er nach wie vor für völlig andere Rahmenbedingungen sorgt.

Die Geschichte der Revision des Aufenthaltsrechts in Deutschland ist wohlbekannt und an anderen Orten gut nachgezeichnet (vgl. Groß 2004; Geddes 2003; Boswell und Geddes 2011; Bergmann u.a. 2016; Klusmeyer und Papademetriou 2013). Die Revision des Staatsbürgerschaftsgesetzes und die Gegenmobilisierung durch CDU-Politiker alter Schule wie Roland Koch, die Ergebnisse der Süssmuth-Kommission und der erste Entwurf eines neuen Aufenthaltsgesetzes ohne Duldungen, die Blockade desselben im Bundesrat und das 2004 verabschiedete, bis heute hin gültige Aufenthaltsgesetz sind vielfach diskutiert worden. Weniger Aufmerksamkeit bekam die Tatsache, dass sich das Migrationsrecht in Deutschland auch seit 2004 unter den Regierungen Merkel weiterentwickelt hat. Einerseits die Umsetzung von EU-Richtlinien, andererseits Weiterführungen des Bleiberechts für Kinder, der Qualifikationsanerkennung, weiter aber auch länderspezifische Initiativen wie Winterabschiebestopps und die effektive Aussetzung von Abschiebehaft als Zwangsmaßnahme haben Migrationsrecht unter dem Strich weiter liberalisiert. Dieses wurde auch durch Entwicklungen auf europäischer Ebene, wie etwa der Ausweitung der vollen Personenfreizügigkeit auf Rumänien und Bulgarien oder wegweisende EGMR-Entscheidungen im Bereich der gleichgeschlechtlichen Partnerschaft verstärkt. Diese Liberalisierung ist abgesehen von Einzelnormen auch über die zunehmend quantitative Metaerhebung von Rechtsveränderungen ersichtlich (vgl. Helbling 2013; De Haas, Natter und Vezzoli 2014; Huddleston et. al. 2016). Auch mit den aktuellen Verschärfungen des Asylrechts ist die rechtliche Situation für Geflüchtete in Deutschland eine deutlich bessere als zu Beginn der Neunzigerjahre. Dies gilt einerseits für Anerkennungsquoten (und die Tatsache, dass das BAMF 2015 nicht aufgehört hat, Fälle zu bearbeiten), andererseits aber auch für viel bessere Bedingungen für die sprachliche und berufliche Integration und eine (relativ, aber immerhin) humanere und grundrechtlich abgesicherte Abschiebepraxis. Dies wirkt sich natürlich unmittelbar auf die Arbeit von Ausländerbehörden aus. 
Ein erster, wichtiger Grund für das veränderte Verhältnis zwischen Ausländerbehörden und Zivilgesellschaft ist die veränderte rechtliche Grundlage, die den Behörden mehr Spielraum für wohlwollende und positive Entscheidungen gibt. Aufenthaltsrecht ist nicht mehr reine ordnungsrechtliche Gefahrenabwehr (selbst die Einflüsse des Terrorismusbekämpfungsgesetzes sind eher gering, vgl. Eule 2014, Kapitel 4), sondern viel mehr Migrationsmanagement, ein nicht besonders systematischer und klar reformbedürftiger, aber deutlich liberalerer Sortierungsmechanismus als vor 25 Jahren. Dass dies nicht selbstverständlich ist, zeigen tendenziell die Entwicklungen in den vormaligen Hochburgen liberalen Einwanderungsrechts in Europa, den Niederlanden und Großbritannien (vgl. Joppke 2014; Banting und Kymlicka 2012; Michalowski 2011) und strukturell ähnlichen Einwanderungsstaaten mit sehr anderen rechtlichen Dynamiken, insbesondere der Schweiz (vgl. Cattacin und Chimienti 2009; Piñeiro, Bopp und Kreis 2009; D'Amato 2012).

\subsection{Der Ausbau der Förderung von Integration über Sprachkurse und Beratungsangebote}

Neben der rechtlichen Reform wurden in Deutschland in großem Maße Gelder in integrationsfördernde Maßnahmen investiert. Dies betrifft vor allem zwei Bereiche: den Ausbau der Sprachförderung über Deutsch- und Integrationskurse sowie den Ausbau der staatlichen Finanzierung von Migrationsberatung. Dies führte dazu, dass viele zivilgesellschaftliche Akteure ihre Angebote für Migrant*innen ausbauen konnten, aber zur Gewinnung von staatlichen Geldern auch professioneller und weniger konfrontativ werden mussten.

Insbesondere der Bereich der Sprachförderung ist hier hervorzuheben. Auch wenn die Einführung von Sprachkenntnissen als Verschärfung der Einreise- und Aufenthaltsbedingungen verstanden werden kann, zeigt die Praxis, dass die sanktionierenden Elemente wenig bis keine Auswirkung haben (vgl. Michalowski und van Oers 2011; Permoser 2012). Umso beachtlicher ist das finanzielle Engagement, das mit der Sprachpflicht einherging. Obwohl Multikulturalismus in Deutschland für gescheitert erklärt wurde, liegen die staatlichen Ausgaben zur Förderung von Integration und Zusammenleben weit über denen anderer Staaten. Allein das so mit seiner Armut kokettierende Berlin gibt dabei jährlich mehr Geld zur Integrationsförderung aus als der gesamte kanadische Staat (vgl. Collett 2011; Joppke und Eule 2016).

Dieses hat sich unmittelbar auf die Struktur der großen zivilgesellschaftlichen Organisationen (und die kleinerer, lokal agierender) ausgewirkt. Migration ist nicht mehr nur ein politisches Kampfthema, sondern ein wichtiger Aufgaben- 
bereich und Einnahmequelle. Viele Akteure sind nun stärker in staatliche Strukturen eingebettet und schon allein zwecks ihres veränderten Auftrages angehalten, nicht nur konfrontativ mit »Staat" umzugehen. Dieses hat geholfen, die internen Dynamiken in den Organisationen zu verändern und so scheinbar zu einer Form von Professionalisierung und Pragmatismus geführt (vgl. Joppke und Eule 2016).

\subsection{Die Veränderung der Struktur und Ausrichtung von Ausländerbehörden}

Die Feldforschung in Ausländerbehörden und Anschlussinterviews mit weiteren Behörden ergaben, dass die Liberalisierung des Migrationsrechts selbst für die Ausländerbehörden unterschiedliche Auswirkungen hatte. Für einen zunächst kleinen Teil von Ausländerbehörden bedeutete die Reform ein tiefgreifendes Umdenken von Arbeitspraktiken und dem Verhältnis zwischen Zugewanderten und Behörde. Dies waren vornehmlich große Städte mit einer langen Tradition zivilgesellschaftlichen Engagements für Zugewanderte und einfachem Zugang zu starken öffentlichen Medien. Ein zweiter, größerer Teil, veränderte vornehmlich das öffentliche Auftreten der Ausländerbehörde gegenüber Medienvertreter*innen und Zivilgesellschaft, ohne aber an der tatsächlichen Arbeitspraxis viel zu reformieren. Ein dritter Teil schließlich lehnte eine institutionelle Veränderung schlicht ab und versuchte, sich von dem sich wandelnden Einwanderungsdiskurs über weitgehende Abschottung zu schützen. Im Laufe der letzten zehn Jahre hat sich die Gruppe der sich abschottenden Ausländerbehörden allerdings stark verkleinert.

Trotz Vollzugsföderalismus ist dabei auffällig, dass hierbei von Bundesebene Anreize geschaffen werden und leichter Druck ausgeübt wird, um die »zögerlichen« Kommunen zu motivieren, über ihren Abschottungsmodus hinaus zu gehen. In Deutschland wurde etwa 2013 ein zweijähriges Modelprojekt »Ausländerbehörden - Willkommensbehörden« initiiert, welches vom Bundesamt für Migration und Flüchtlinge koordiniert, aber von den Ländern finanziert wird. Hierbei dienen Behörden, die »Willkommenskultur« - also eine Form der Übernahme von liberalen rechtlichen Ideen in Praxis und Arbeitsstruktur - bereits erfolgreich umgesetzt haben, als Vorbilder und profitieren gleichzeitig von dem Austausch mit den Konzepten anderer Behörden (vgl. Eule 2015).

Auch wenn die tatsächlichen Auswirkungen solcher Projekte noch nicht abschätzbar sind - und nicht zu hoch angesetzt werden sollten - ist doch auffällig, dass diese Debatte inzwischen nicht nur öffentlich, sondern vor allem in Praktikerkreisen geführt wird. Auch wenn längst nicht alle der über 400 Ausländerbe- 
hörden »Willkommensbehörden« sind oder sein werden, und auch wenn die Bedeutung von »Willkommensbehörden« in der Regel nicht »Willkommen«, sondern »Auswahl« ist, auch wenn die beforschten Ausländerbehörden eher durch Informalität und Chaos als durch Professionalisierung und Integrationsfreude glänzten, ist eine solche Strukturdebatte nicht grundsätzlich abzutun, denn sie ist neu und hat zumindest bei den »Vorreiterbehörden« enorme Auswirkungen gehabt.

\section{Migrationssteuerung als dynamisches Feld}

Das vorliegende Kapitel hat versucht einen kleinen, aber wichtigen Punkt zu vermitteln: Akteure der Migrationssteuerung und Zivilgesellschaft stehen sich mitnichten grundsätzlich antagonistisch gegenüber, sondern sind zunehmend in nicht reibungsfreien, aber funktionalen und relativ vertrauensvollen Zweckbeziehungen. Ein Blick in die tatsächliche Arbeitspraxis zeigt, dass starre funktionsbezogene Darstellungen nicht ausreichen, um das komplexe und überaus dynamische Miteinander zu verstehen. Auch wenn Ausländerbehörden keine Willkommensbehörden sind, haben sie durchaus vielfach den Willen und die Möglichkeiten, im Rahmen ihres Ermessens (und darüber hinaus) wohlwollend und mit zivilgesellschaftlichen Akteuren gemeinsam Lösungen für Einzelfälle zu finden. Auch wenn zivilgesellschaftliche Organisationen sich primär als Unterstützer von Zugewanderten verstehen, scheinen sie inzwischen so professionalisiert und staatlich eingebunden, dass sie pragmatischen Entscheidungen offen gegenüber stehen und die Grenzen geltenden Rechts als Grenzen ihrer eigenen Mobilisierung für Einzelfälle anerkennen. Auch wenn Vertreter*innen von Zivilgesellschaft und Ausländerbehörden nicht immer einer Meinung sind, können sie sich gegenseitig unterstützen - selbst, wie wir gesehen haben, gegen eigentlich »artverwandte« Stellen und Behörden.

Dies stellt einerseits die Frage, in wie weit zwischen »staatstragenden« und kritischeren zivilgesellschaftlichen Fraktionen unterschieden werden muss - zumindest einige Interviews zu Beginn der Feldforschung scheinen dies zu bestätigen. Viel wichtiger im Kontext der Frage des Verwaltens von Asyl ist aber, wie wir die Ausgangslage verstehen, in der in den kommenden Monaten und Jahren Konflikte um Einzelfälle und Migrationspolitik im Allgemeinen ausgetragen werden. Die Erfahrung der Feldforschung zeigt eindeutig, wie anders die Praxis im Vergleich zur letzten »Flüchtlingskrise« sich verhält. Dies hat mit rechtlichen Veränderungen, finanziellen Investitionen in Integrationsförderung durch Zivilgesellschaft und strukturellen Reformen in Ausländerbehörden zu tun. Bei aller 
berechtigten Kritik an logistischen Verfehlungen, Rechtsverschärfungen, sicheren Herkunftsländern, Abschiebeplänen und vielem Weiteren, sollten die viel positiveren Grundbedingungen, unter denen Geflüchtete unterstützt und in sicherere, integrierende Lösungen gebracht werden können, nicht übersehen werden.

Migrationssoziologisch gesehen kann man das Kapitel schließlich als Ruf für weitere akteursspezifische Forschung verstehen, die der hier vorgestellten Dynamik weitere Nuancen und Datenfülle verleiht. Praxis zu ignorieren oder in Schwarz/Weiß-Verhältnissen zu karikieren, ist nicht nur nach vorliegender Datenlage falsch, sondern auch fatal: Es sind die lokalen Figurationen, in denen zivilgesellschaftliches Engagement am meisten auslösen kann, und die vertrauteren, institutionalisierteren und regen Verbindungen zwischen Zivilgesellschaft und Ausländerbehörden können hier eine wichtige Ressource sein, von der aus politisches, soziales und wissenschaftliches Engagement starten kann.

\section{Literatur}

Anderson, Philip (2011): „Die Wahrung der Menschenrechte von MigrantInnen in der Illegalität auf kommunaler Ebene - das Beispiel München«, in: Maren Mylius/Wiebke Bornschlegl/Andreas Frewer (Hg.), Medizin für Menschen ohne Papiere. Menschenrecht und Ethik in der Praxis des Gesundheitssystems (= Medizin und Menschenrechte, Band 5), Göttingen: V\&R Unipress, S. 173-196.

Banting, Keith/Kymlicka, Will (2012): Is There Really a Backlash Against Multiculturalism Policies?, Stockholm: SULCIS.

Bergmann, Jan/Dienelt, Klaus/Bauer, Ina/Samel, Kai-Christian/Sußmann, Alexandra/Winkelmann, Holger/Wunderle, Simone/Kanein, Werner/u.a. (2016): Ausländerrecht: Aufenthaltsgesetz, Freizügigkeitsgesetz/EU und ARB 1/80, 11. Auflage, München: C.H. Beck.

Borgards, Vera (2006): »Menschen in der Illegalität und die Praxis der Rechtsausübung«, in: Jörg Alt/Michael Bommes (Hg.), Illegalität, Wiesbaden: VS Verlag für Sozialwissenschaften, S. 157-164.

Boswell, Christina/Geddes, Andrew (2011): Migration and mobility in the European Union, Basingstoke: Palgrave Macmillan.

Buckel, Simone (2011): »Urban Governance und irreguläre Migration: Städtische Politik als Handlungsraum im Konfliktfeld irreguläre Migration«, in: Oliver Frey/Florian Koch (Hg.), Die Zukunft der Europäischen Stadt, Wiesbaden: VS Verlag für Sozialwissenschaften, S. 246-262. 
Castles, Stephen (2004a): »The Factors That Make and Unmake Migration Policies«, in: The International Migration Review 38, S. 852-884.

Castles, Stephen (2004b): »Why migration policies fail«, in: Ethnic and Racial Studies 27, S. 205-227.

Cattacin, Sandro/Chimienti, Milena (2009): »Lokale Politik der Eingliederung der Migrationsbevölkerung in der Schweiz - Zwischen Pragmatismus und Populismus«, in: Frank Gesemann/Roland Roth (Hg.), Lokale Integrationspolitik in der Einwanderungsgesellschaft. Migration und Integration als Herausforderung von Kommunen, Wiesbaden: VS Verlag für Sozialwissenschaften, S. 655-672.

Collett, Elizabeth (2011): Immigrant Integration in Europe in a Time of Austerity, Bruxelles: Migration Policy Institute.

Côté-Boucher, Karine/Infantino, Federica/Salter, Mark B. (2014): »Border security as practice: An agenda for research «, in: Security Dialogue 45, S. 195-208.

Cyrus, Norbert/Vogel, Dita (2003): »Work-permit decisions in the German labour administration: an exploration of the implementation process «, in: Journal of ethnic and migration studies 29, S. 225-256.

D’Amato, Gianni (2012): »Switzerland«, in: Christian Joppke/F. Leslie Seidle (Hg.), Immigrant integration in federal countries (= Thematic issues in federalism, Band 2), Montréal: McGill-Queen's University Press, S. 162-191.

De Haas, Hein/Natter, Katharina/Vezzoli, Simona (2014): Growing restrictiveness or changing selection? The nature and evolution of migration policies, Oxford: IMI.

Ellermann, Antje (2009): States Against Migrants: Deportation in Germany and the United States, Cambridge: Cambridge University Press.

Eule, Tobias G. (2014): Inside Immigration Law: Migration Management and Policy Application in Germany, Farnham: Ashgate.

Eule, Tobias G. (2015): »Willkommenskultur auf dem Prüfstand «, in: Christian Joppke/Ben Jann/Axel Franzen (Hg.), Essays on Inequality and Integration. Zürich: Seismo, S. 95-109.

Feldman, Gregory (2012): The migration apparatus: security, labor, and policymaking in the European Union, Stanford: Stanford University Press.

Geddes, Andrew (2003): The Politics of Migration and Immigration in Europe, London: SAGE Publications.

Geiger, Martin/Pécoud, Antoine (Hg.) (2010): The New Politics of Migration Management: Actors, Discourses and Practices, Berlin: Springer.

Geiger, Martin/Pécoud, Antoine (Hg.) (2012): The new politics of international mobility migration management and its discontents, Osnabrück: IMIS. 
Groß, Thomas (2004): »Germany«, in: Imelda Higgins/Kay Hailbronner (Hg.), Migration and asylum law and policy in the European Union, FIDE 2004 national reports, Cambridge: Cambridge University Press, S. 111-134.

Helbling, Marc (2013): »Validating integration and citizenship policy indices«, in: Comparative European Politics 11, S. 555-576.

Hollifield, James (2004): »France: Republicanism and the Limits of Immigration Control«, in: Wayne A. Cornelius (Hg.), Controlling Immigration: A Global Perspective, Stanford: Stanford University Press, S. 157-187.

Huddleston, Thomas/Bilgili, Ozge/Joki, Anne-Linde/Vankova, Zvezda (2016): MIPEX 2015: Germany, Migration Integration Policy Index.

Janis, Irving L. (1972): Victims of Groupthink. A Psychological Study of Foreign-Policy Decisions and Fiascoes, Boston: Houghton, Mifflin.

Joppke, Christian (2014): »European Immigrant Integration after Multiculturalism«, in: Graziano Battistella (Hg.), Global and Asian Perspectives on International Migration, Cham: Springer International Publishing, S. 77-99.

Joppke, Christian/Eule, Tobias G. (2016): „Civic integration in Europe: continuity versus discontinuity«, in: Gary P. Freeman/Nikola Mirilovic (Hg.), Handbook on migration and social policy, Cheltenham: Edward Elgar, S. 342-361.

Klusmeyer, Douglas B./Papademetriou, Demetrios G. (2013): Immigration Policy in the Federal Republic of Germany: Negotiating Membership and Remaking the Nation, Oxford: Berghahn Books.

Michalowski, Ines (2011): »Required to assimilate? The content of citizenship tests in five countries «, in: Citizenship Studies 15, S. 749-768.

Michalowski, Ines/van Oers, Ricky (2011): »How Can We Categorise and Interpret Civic Integration Policies? «, in: Journal of Ethnic and Migration Studies 38, S. 163-171.

Morris, Lydia (2002): Managing Migration: Civic Stratification and Migrants' Rights, London: Routledge.

Permoser, Julia Mourão (2012): „Civic Integration as Symbolic Politics: Insights from Austria«, in: European Journal of Migration and Law 14, S. 173-198.

Piñeiro, Esteban/Bopp, Isabelle/Kreis, Georg (Hg.) (2009): Fördern und Fordern im Fokus. Leerstellen des schweizerischen Integrationsdiskurses, Zürich: Seismo.

Scheffer, Thomas (1995): Aufenthaltsgenehmigung - Studien zur Praxis der Ausländerverwaltung, Bielefeld: Fakultät für Soziologie.

Scheffer, Thomas (2001): Asylgewährung, Stuttgart: Lucius \& Lucius. 


\title{
Die Entstehung rechtlicher Fallgeschichten in einem Übersetzungsprozess
}

Die Rechtsvertretung asylsuchender Personen

in einem schweizerischen Hilfswerk

JOHANNA FUCHS

\section{Einleitung}

\begin{abstract}
»Wir, die Anwälte, [...] haben die Rolle, Menschen zu vertreten; wir haben die Rolle, das, was uns die Menschen erzählen, für das juristische Verfahren in Form zu bringen. Deshalb können wir sagen, es ist eine Übersetzungsarbeit im weit verstandenen Sinn des Wortes. $\aleph^{1}$
\end{abstract}

So beschrieb ein auf Asylrecht spezialisierter Anwalt seine Arbeit in einem Interview. In diesem Beitrag gehe ich auf die Rolle der Rechtsvertreter_innen von asylsuchenden Personen ein. Ich zeige, wie sie an der Schnittstelle zwischen den Behörden und den Asylsuchenden stehen. Die Rechtsvertreter_innen sind Vermittler_innen oder, in den Worten des zitierten Anwalts, Übersetzer_innen. Auf der einen Seite erklären sie den Asylsuchenden das Asylsystem und den Verfahrensablauf. Auf der anderen Seite formulieren die Rechtsvertreter_innen die Anliegen der Asylsuchenden so, dass sie bei den Behörden und Gerichten Gehör finden.

1 Von der Autorin aus dem Französischen übersetzt. 
Ein großer Teil der Arbeit der Rechtsvertreter_innen besteht darin, schriftliche Gesuche und Beschwerden an die Behörden und Gerichte zu schreiben. Sie versuchen zum Beispiel für ihre Klient_innen mittels einer Beschwerde an das Bundesverwaltungsgericht (BvGer) eine Aufenthaltserlaubnis in der Schweiz zu erlangen. Die Rechtsvertreter_innen stellen Gesuche um Familienzusammenführung an kantonale Migrationsämter oder sie helfen den Asylsuchenden, einen Visumsantrag an das Staatssekretariat für Migration (SEM) zu stellen.

Ein wichtiger Bestandteil dieser Gesuche und Beschwerden sind die Fallgeschichten $^{2}$ ihrer Klient_innen. In den schriftlichen Dokumenten wird die individuelle Fallgeschichte der asylsuchenden Person, der Sachverhalt, beschrieben und mit der rechtlichen Kategorie ${ }^{3}$ verbunden. Im Mittelpunkt dieses Beitrags steht der Prozess, in dem diese Fallgeschichten von den Rechtsvertreter_innen vorbereitet und verfasst werden.

Das Recht setzt eine bestimmte Wissensdarstellung voraus, der die Fallgeschichten entsprechen müssen, damit sie für die Behörden und Gerichte fassbar sind (vgl. Conley/O'Barr 1990; Mertz 2007; Merry/Coutin 2014; Coutin/ Mallin/Merry 2014). Die Rechtsvertreter_innen formen die Fallgeschichten deshalb in den Rechtsschriften so, dass sie innerhalb des rechtlichen Wissenssystems bestehen können. Ich zeige in diesem Beitrag auf, wie die Fallgeschichten von den Rechtsvertreter_innen kreativ und strategisch je nach rechtlicher Kategorie anders dargestellt werden (vgl. McKinley 1997). Das Konzept der Übersetzung dient mir dazu, den Prozess zu beschreiben, in dem die Fallgeschichten von den Rechtsvertreter_innen hergestellt werden (vgl. Rottenburg 2002). In Anlehnung an Merry und Coutin gehe ich in diesem Beitrag folgenden Fragen nach (Merry/Coutin 2014: 3; Coutin/Mallin/Merry 2014: 7): Wie wird der Sachverhalt je nach rechtlicher Kategorie von den Rechtsvertreter_innen dargestellt? Was wird von den Rechtsvertreter_innen sichtbar gemacht und was weggelassen? $\mathrm{Zu}$ welchen Handlungen raten die Rechtsvertreter_innen ihren Klient_innen, um ihre Fallgeschichten vorzubereiten und zu belegen?

Anhand eines empirischen Beispiels zeige ich, wie die Rechtsvertreter_innen versuchen, für Frau G einen dauerhaften Aufenthaltstitel in der Schweiz zu er-

2 Unter »Fallgeschichte« verstehe ich den Sachverhalt eines individuellen Falles, der in einer Rechtsschrift beschrieben wird. Mertz verwendet den Begriff der »legal story« oder »legal narrative« (vgl. Mertz 2007).

3 Unter »rechtlicher Kategorie« verstehe ich die Gesetzesartikel (oder eine Kombination von Gesetzesartikeln) und die damit verbundene Rechtsprechung und -lehre, die vorgeben, welche Kriterien erfüllt sein müssen, damit zum Beispiel eine Person einen Flüchtlingsstatus erhält oder eine Familienzusammenführung bewilligt wird. 
halten, nachdem ihr Asylgesuch von den Behörden erstinstanzlich abgelehnt wurde. Ich folge der Arbeit der Rechtsvertreter_innen am Beispiel von Frau G über einen Zeitraum von sechs Monaten. Ich beschreibe, wie die Rechtsberater_innen während dieser Zeit drei unterschiedliche Darstellungen der Fallgeschichte von Frau G herstellen. Drei rechtliche Kategorien setzen drei verschiedene Fallgeschichten voraus. Je nach rechtlicher Kategorie werden gewisse Elemente aus ihrer Erzählung und ihren Akten zentral und andere irrelevant. Ich führe aus, wie das rechtliche Wissenssystem eine lückenlose Fallgeschichte von Frau $\mathrm{G}$ voraussetzt. Widersprüchliche und verschwommene Aspekte versuchen die Rechtsvertreter_innen zu glätten, indem sie diese Aspekte nicht erwähnen und den Fokus auf Elemente legen, die ihnen eindeutiger erscheinen. Ich lege erstens dar, wie die drei entstandenen Fallgeschichten von Frau G nie vollständig in die jeweilige legale Kategorie integrierbar sind. Es gelingt den Rechtsberater_innen nicht, die Lücken zu schließen und mit ihrer Fallgeschichte alle Kriterien zu erfüllen, die von der rechtlichen Kategorie vorausgesetzt werden. Zweitens argumentiere ich, dass die unterschiedlichen Fallgeschichten, die von den Rechtsberater_innen produziert werden, auch untereinander nicht vereinbar sind. Sie widersprechen sich und sind gleichzeitig voneinander abhängig.

Die Ausführungen gliedern sich in mehrere Schritte. Im ersten Kapitel dieses Beitrags beschreibe ich den Kontext der Feldforschung und die Methode. Im zweiten Teil lege ich die theoretische Grundlage dar, die mir zur Analyse des empirischen Beispiels dient. Anhand des Beispiels von Frau G zeige ich in drei Unterkapiteln, wie die Rechtsvertreter_innen drei Darstellungen ihrer Fallgeschichte produzieren. In der ersten Geschichte entsteht ein Bild von Frau G als alleinstehende verletzliche Mutter in einer patriarchalen Gesellschaft, die Opfer sexueller Gewalt wurde. In der zweiten Übersetzung wechseln die Rechtsvertreter_innen den Fokus auf die Bemühungen von Frau G, sich in der Schweiz zu integrieren und auf die Schulkarriere ihrer Tochter. Schließlich treten in der dritten Fallgeschichte die standesamtliche Heirat von Frau G mit dem Vater ihrer Tochter und die Vaterschaftsanerkennung in den Mittelpunkt, welche dazu dienen sollten, eine Familienzusammenführung zu erreichen. Im Schlusswort gehe ich erneut auf den Begriff der Übersetzung ein. Ich komme dabei auf das Spannungsverhältnis zwischen den drei unterschiedlichen Darstellungen des Falles von Frau $G$ zurück. Ich zeige, wie diese drei Fallgeschichten in ungleichen Machtverhältnissen entstehen. 


\section{Feldforschung in einer Beratungsstelle für asylsuchende Personen}

Stellt eine Person in der Schweiz ein Asylgesuch, wird sie in einem Empfangsund Verfahrenszentrum registriert. In der Folge interviewt ein_e Beamt_in des SEM die Asyl beantragende Person in zwei Anhörungen ${ }^{4} \mathrm{zu}$ den Asylgründen. Aufgrund dieser Interviews wird vom SEM ein Entscheid gefällt. Es wird begründet, ob und warum die Person in der Schweiz bleiben darf oder nicht. Falls der Entscheid positiv ausfällt, wird die Art des Aufenthaltstitels bestimmt. Der Entscheid wird der asylsuchenden Person schriftlich eröffnet. Bei einer Ablehnung hat sie die Möglichkeit, in einer Frist von dreißig Tagen ${ }^{5}$ eine Beschwerde beim BvGer zu erheben.

Die Anhörungen sind meistens die einzigen Momente, in denen die asylsuchenden Personen in direkten Kontakt mit den entscheidungsberechtigten Behörden kommen und die Gelegenheit haben, ihr Anliegen mündlich vorzutragen. Die Beschwerde und andere rechtliche Verfahren werden ausschließlich schriftlich abgewickelt. Da die asylsuchenden Personen sich in der Regel im schweizerischen Rechtssystem nicht auskennen und sich nicht in einer für die Behörden und Gerichte verständlichen Sprache ausdrücken können, sind sie auf die Hilfe anderer Personen angewiesen. Kommt es zu einem Beschwerdeverfahren oder zu zusätzlichen rechtlichen Anträgen, werden die meisten Asylsuchenden deshalb von juristischen Fachpersonen beraten und vor den Behörden und Gerichten vertreten. Die Rechtsvertreter_innen schreiben Briefe, Beschwerden und andere rechtliche Eingaben. Diese von den Rechtsvertreter_innen verfassten Dokumente beschränken sich nicht nur auf das Asylrecht, sondern sie beziehen sich auch auf verschiedene Rechtstexte, zum Beispiel auf das Ausländer- und Familienrecht. Sowohl nationale als auch internationale Gesetzgebungen werden von den Rechtsvertreter_innen in ihrer Arbeit herangezogen, um ihre Klient_innen zu vertreten. Auch im später dargelegten empirischen Beispiel verwenden die Rechtsvertreter_innen eine Fülle an verschiedenen Gesetzestexten.

Neben kostenpflichtigen herkömmlichen Anwaltskanzleien gibt es in der Schweiz in jedem Kanton mindestens ein nichtstaatliches Hilfswerk, das auf die

4 Die asylsuchende Person wird in der sogenannten Befragung zur Person (BzP) kurz zu ihren Asylmotiven befragt. Es werden hauptsächlich Angaben zu ihren Personalien, zum Fluchtweg etc. gesammelt. In einer längeren zweiten Anhörung, der Bundesanhörung, wird die Person ausführlich zu ihren Asylgründen befragt. Beide Interviews werden protokolliert.

5 Je nach rechtlicher Situation kann die Frist auch nur fünf Arbeitstage betragen. 
Rechtsberatung und -vertretung im Asyl- und Ausländerrecht spezialisiert ist. ${ }^{6}$ Die Hilfswerke gelten als unabhängig von Bund und Kantonen. Sie bieten ihre Dienste kostenlos ${ }^{7}$ an und werden zu einem großen Teil durch private Spenden finanziert. Oft leisten auch Gemeinden und Kantone einen finanziellen Beitrag in Form einer Spende. Die einzelnen kantonalen Organisationen sind unabhängig voneinander. Ihre Entstehungsgeschichten und Organisationsstrukturen sind unterschiedlich. Sie sind aber in einem nationalen Netzwerk zusammengeschlossen, das von der Schweizerischen Flüchtlingshilfe ${ }^{8}$ koordiniert wird. Vertreter_innen aller kantonalen Organisationen treffen sich einmal monatlich, um sich über juristische Fälle auszutauschen.

In den Hilfswerken arbeiten zum größten Teil Personen mit einer juristischen Ausbildung. Die wenigen Nichtjurist_innen eignen sich das rechtliche Fachwissen auf anderen Wegen an. Die Arbeitsbelastung ist oft sehr hoch und die finanziellen Mittel der Organisationen sind begrenzt. Dies ist einer der Gründe, weshalb in einigen Organisationen auch Freiwillige mitarbeiten. Sie übernehmen ebenfalls Mandate für die asylsuchenden Personen. Oft sind die freiwilligen Mitarbeiter_innen Student_innen der Rechtswissenschaften oder einer verwandten Studienrichtung. Ich sehe sie deshalb ebenfalls als juristische Fachpersonen an. ${ }^{9}$

6 Beispiele dieser Hilfswerke sind »Caritas Schweiz«, »Hilfswerk der evangelischen Kirchen Schweiz« oder »Service d'aide juridique aux exilé-e-s«.

7 In manchen Beratungsstellen wird auch ein geringer finanzieller Beitrag von den asylsuchenden Personen verlangt.

8 Die Organisation Schweizerische Flüchtlingshilfe kann mit Pro Asyl in Deutschland verglichen werden.

9 Ich sehe sowohl die freiwilligen als auch die festangestellten Rechtsvertreter_innen in den Hilfswerken als juristische Fachpersonen an. Doch ist es wichtig zu beachten, dass die juristische Vertretung in den Hilfswerken kostenlos angeboten wird. Mit der Vermischung von rechtlichem Fachwissen und humanitärer Hilfe befassten sich zum Beispiel Nicolas Fischer und Estelle d'Halluin-Mabillot in ihren empirischen Forschungen. Sie untersuchten im französischen Kontext, wie Rechtsberatungsstellen von Hilfswerken sich mittels des Rechts gegen die Asylbehörden widersetzten. Fischer zeigt die Vermischung der verschiedenen Aufgaben der Rechtsvertreter_innen auf: Einerseits bieten sie Hilfe und Widerstand gegen eine staatliche Migrationspolitik. Andererseits schränkt sie der rechtliche Rahmen in ihrem Tun ein (Fischer 2009). Auch Estelle d'Halluin-Mabillot erforscht, wie sich die Rechtsvertreter_innen in Hilfswerken und Vereinen zwischen einer humanitären Motivation und dem Willen zur Professionalität hin und her bewegen. Sie zeigt auf, wie die freiwilligen Mitarbeiter_innen in diesen Organisationen eine andere Auffassung ihrer Rolle haben als die 
Haben die asylsuchenden Personen rechtliche Fragen oder benötigen sie eine Rechtsvertretung, wenden sie sich meistens an eines dieser Hilfswerke. Ein wichtiger Grund dafür ist, dass die asylsuchenden Personen oft mittellos sind und die Rechtsberatung und -vertretung in diesen Organisationen kostenlos ist. Zudem gelten die in der Schweizerischen Flüchtlingshilfe zusammengeschlossenen Hilfswerke als kompetente Akteure im schweizerischen Asylbereich. Zum Beispiel vermitteln die Sozialarbeiter_innen in den Wohnheimen die Asylsuchenden an die beschriebenen Beratungsstellen. ${ }^{10}$ Obwohl sie für den Verlauf des asylrechtlichen Verfahrens wichtig sind, ist die Arbeit dieser Organisationen im schweizerischen Kontext in der bisherigen Forschung wenig bearbeitet worden. ${ }^{11}$ Diese Lücke möchte der vorliegende Beitrag schließen helfen.

Die Analyse in diesem Beitrag basiert auf empirischem Datenmaterial, das ich für meine laufende Forschung zu meiner Dissertation in einem dieser Hilfswerke während sieben Monaten in den Jahren 2014 und 2015 gesammelt habe. ${ }^{12}$ Ich arbeitete als freiwillige Mitarbeiterin in diesem Hilfswerk und hatte so die Möglichkeit, bei allen alltäglichen Arbeitsschritten der Rechtsvertreter_innen beteiligt zu sein: Ich beobachtete Beratungsgespräche zwischen Rechtsvertreter_innen und Asylsuchenden. Ich führte selber zahlreiche Beratungsgespräche mit Asylsuchenden. Die aus den Beratungsgesprächen hervorgehende Aktenarbeit führte ich in enger Absprache mit meinen Kolleg_innen selbständig aus. Ich schrieb Beschwerden und andere administrative Eingaben an das BvGer und an die Migrationsbehörden. Ich hatte dabei Einsicht in die Asylakten der Klient_innen. Ich war in Kontakt mit kantonalen und nationalen Migrationsämtern,

festangestellten juristischen Fachpersonen (d'Halluin-Mabillot 2012). Auf diesen Aspekt der Arbeit der Rechtsvertreter_innen werde ich in meiner Dissertationsforschung näher eingehen.

10 Die Schweizerische Flüchtlingshilfe ist zudem am politischen Konsultationsprozess über die Asyl- und Migrationsgesetzgebung beteiligt. Das verdeutlicht ebenfalls den wichtigen Stellenwert dieser Organisationen im schweizerischen Asylsystem.

11 Eine Ausnahme ist der Aufsatz von Raphaël Rey und Olivier Beurret. Die Autoren untersuchten empirisch, wie die Rechtsvertreter_innen Fälle auswählen, für die sie Beschwerde beim BvGer einreichen. Die Sozialanthropologen beschreiben, wie die Rechtsvertreter_innen diese Entscheidung im Dilemma fällen, den Asylsuchenden einerseits eine Dienstleistung und Hilfe anbieten zu wollen. Gleichzeitig fühlen sie sich aber verpflichtet, dem Recht und ihrer damit verbundenen beruflichen Professionalität Rechnung zu tragen (vgl. Rey/Beurret 2013).

12 Aus Vertraulichkeitsgründen nenne ich im Folgenden das Hilfswerk, in dem ich Feldforschung führte, »das Hilfswerk«, »die Beratungsstelle« oder »das Beratungsbüro«. 
Gerichten, anderen Hilfsorganisationen, mit Ärzt_innen und mit Sozialarbeiter_innen der Asylsuchenden. Innerhalb des Beratungsbüros nahm ich an internen Sitzungen und an Supervisionen teil. Neben der Feldforschung innerhalb der Beratungsstelle war ich auch bei nationalen Treffen zwischen verschiedenen Beratungsbüros dabei. Ich besuchte mehrere juristische Weiterbildungen zum Asylrecht.

Mit meiner freiwilligen Mitarbeit in der Rechtsberatungsstelle entschied ich mich für eine aktiv beobachtende Teilnahme im Feld (vgl. DeWalt/DeWalt 2011: 24). Im empirischen Fallbeispiel bin ich nicht nur die beobachtende Forscherin, sondern vertrete Frau G auch in ihrem Asylverfahren. Durch meine aktive Rolle als Freiwillige im Beratungsbüro bekam ich eine umfassende Innenansicht des Arbeitsalltags. Als eine der ihren teilte ich ihre Anliegen und Sorgen. Da ich die Arbeitsschritte mit meinen Kolleg_innen vor- und/oder nachbesprach, ergaben sich zahlreiche informelle Gespräche aufgrund konkreter Fallbesprechungen. Sie reichten oft über die Besprechung des spezifischen Falles hinaus. Allgemeinere Themen der Rechtsberatungsarbeit mit Asylsuchenden kamen so zur Sprache.

Wie Staci Newmahr ausführt, sehe ich meine eigenen subjektiven Erfahrungen, die ich als freiwillige Rechtsberaterin machte, als Möglichkeit, ein soziales Phänomen - die Rechtsvertretung von asylsuchenden Personen - besser zu verstehen. Indem ich meine subjektiven Erfahrungen mit dem verbinde, was meine Kolleg_innen im Hilfswerk erzählen und tun, wird es für mich als Forscherin möglich, eine Innenansicht in die Rechtsvertretung von asylsuchenden Personen in Hilfswerken zu erlangen (vgl. Newmahr 2008: 640).

Während der Beratungsgespräche mit den asylsuchenden Personen und in den Gesprächen mit meinen Kolleg_innen im Feld war es mir in erster Linie wichtig, meine Klient_innen juristisch kompetent zu beraten und vor den Behörden und den Gerichten zu vertreten. Eine kritische Distanz und Reflexivität gegenüber den Geschehnissen im Feld war deshalb manchmal schwer aufrecht zu erhalten. Während des Schreibens der Feldnotizen rückte meine Rolle als Forscherin wieder in den Vordergrund und ich konnte eine Distanz zum Feld und meiner Rolle als freiwillige Rechtsberaterin zurückerhalten.

Das empirische Beispiel in diesem Beitrag basiert auf meinen Feldtagebüchern. Ich hatte zudem Einsicht in die Akten von Frau G. Ich beziehe auch die von mir selbst verfassten juristischen Dokumente in meine Analyse ein. Diese Dokumente verfasste ich im Rahmen der Vertretung von Frau G. Die Beschwerde und andere Dokumente wurden von meinen Kolleg_innen gegengelesen und kommentiert. Auch alle Beratungsschritte sprach ich mit meinen Kolleg_innen 
ab. ${ }^{13}$ Die Analyse des Datenmaterials folgt der Grounded Theory Methode von Glaser und Strauss (2010).

\section{3. Übersetzungsprozesse und Recht}

Damit eine Fallgeschichte für die Behörden und die Gerichte fassbar wird und sie über das Gesuch oder die Beschwerde entscheiden können, muss die Geschichte sowohl inhaltlich als auch formal der Wissensdarstellung entsprechen, die dem Recht eigen ist (vgl. Conley/O’Barr 1990; Yngvesson 1993; Mertz 2007; Merry/Coutin 2014; Coutin/Mallin/Merry 2014). Elizabeth Mertz beschreibt, wie Recht bestimmt, was in einer Fallgeschichte erzählt und in welcher Weise es dort dargestellt wird: »In each case, a combination of procedural and doctrinal or similar legal warrants delimits which versions of what occurred (and indeed which aspects of the events in question) will be included in a set of legal facts. « (Mertz 2007: 67)

Die rechtliche Wissensdarstellung ${ }^{14}$ unterscheidet sich von der Alltagssprache (vgl. Conley/O’Barr 1990; Yngvesson 1993; Mertz 2007). Im Prozess, in dem eine Fallgeschichte entsteht, haben die Rechtsvertreter_innen der Asylsuchenden eine Schlüsselrolle inne (vgl. Merry 2006; Coutin 2003). Denn die Rechtsberater_innen sind juristische Fachpersonen und können sich in einer Sprache ausdrücken, die von den Behörden und Gerichten verstanden wird. Als »Menschen in der Mitte« oder »Vermittler_innen« haben die Rechtsberater_innen die Aufgabe, Ideen und Vorstellungen je nach Kontext anzupassen und umzudefinieren (vgl. Merry 2006: 39). Von dieser Annahme ausgehend untersuchen Conley und O'Barr und andere Forscher_innen empirisch, wie Anwält_innen und andere juristische Fachpersonen während des Rechtsverfahrens

13 Das Besprechen von komplexen Fällen zwischen den Rechtsvertreter_innen gehört zum Arbeitsalltag. Vor allem die freiwilligen Mitarbeiter_innen des Beratungsbüros sprechen die Beratungsschritte meistens mit erfahreneren Berater_innen ab und lassen ihre schriftlichen Dokumente korrigieren, bevor sie sie an eine Behörde oder an ein Gericht schicken. Aus meiner Perspektive als Forscherin hatte dieses Besprechen von Fällen einen analytischen Vorteil, da es mir ermöglichte, mein eigenes Handeln aus der Sicht einer Rechtsvertreterin zu reflektieren.

14 Ich gebrauche den Begriff »rechtliches Wissenssystem« oder »rechtliche Wissensdarstellung « im Sinne von Merry und Coutin: »Legal and technical knowledge systems thus make particular versions of social reality visible, even as they obscure others, rendering them unknowable.« (Merry/Coutin 2014: 3) 
die alltagssprachlichen Erzählungen ihrer Klient_innen in »regel-orientierte Erzählungen« für die Gerichte und Behörden formen (vgl. Felstiner/Abel/Sarat 1980; Yngvesson 1993; Mertz 2007; Merry/Coutin 2014).

Ein großer Teil der Arbeit der Rechtsvertreter_innen von asylsuchenden Personen besteht darin, ihre Klient_innen vor den kantonalen und nationalen Migrationsämtern oder vor dem BvGer rechtlich zu vertreten und schriftliche Eingaben zu verfassen. In diesen schriftlichen Dokumenten stellen die Rechtsberater_innen die Fallgeschichten ihrer Klient_innen so dar, dass sie in den Worten von Conley und O'Barr »regel-orientiert« sind, also den Anforderungen der rechtlichen Wissensdarstellung entsprechen (vgl. Coleny/O'Barr 1990). Den Prozess, in dem rechtliche Fallgeschichten im Asylbereich entstehen, haben frühere Studien bereits empirisch untersucht. Tommaso Sbriccoli und Stefano Jacoviello erforschen, wie Rechtsvertreter_innen die Erzählung von Asylsuchenden in einen formalen legalen Rahmen setzen und wie durch diesen Transfer eine neue Geschichte entsteht (vgl. Sbriccoli/Jacoviello 2011). Michelle McKinley beschreibt, wie Anwält_innen sich die Geschichten ihrer Klient_innen aneignen und sie so formen, dass sie im Rechtsverfahren strategisch gebraucht werden können: Die Rechtsvertreter_innen stellen die individuellen Erfahrungen der Asylsuchenden möglichst tragisch dar und fügen sie in stereotype Formen von Migration, von Frauen und von Kultur ein. In den Gerichtsverfahren besteht kein Platz für Widersprüche und für Zwischentöne in den Erzählungen der Migrant_innen (vgl. McKinley 1997). ${ }^{15}$

In diesem Beitrag gehe ich ebenfalls darauf ein, wie die von den Rechtsberater_innen in der Rechtsschrift dargelegte Fallgeschichte entsteht. An einem empirischen Beispiel zeige ich auf, wie die Fallgeschichten das Produkt eines Prozesses sind, der nichtlinear im Dialog zwischen einer Vielzahl von Akteur_innen stattfindet. Ich verwende den Begriff der Übersetzung, um den Prozess zu beschreiben, in dem die für die Behörden und Gerichte fassbaren Fallgeschichten entstehen. Der Begriff der Übersetzung stellt das Dialogische und Prozesshafte in den Mittelpunkt. Richard Rottenburg beschreibt den Vorgang des Übersetzens als eine Praktik, die »Getrenntes zusammenbringt«, sie »vermittelt zwischen zwei Elementen« und macht sie »vergleichbar« (Rottenburg 2002: 15). Während

15 Dies trifft auch auf Rechtsverfahren zu, die nicht spezifisch mit dem Asyl- und Migrationsrecht zu tun haben. So untersuchte Alessandra Gribaldo zum Beispiel Gerichtsverfahren von Frauen in Italien, die häusliche Gewalt erlebt haben. Sie zeigt, wie die Aussagen der Frauen vor Gericht in den diskursiven Rahmen des Rechts passen müssen und so die spezifische Form der Subjektivität »Frau-als-Opfer« entsteht (vgl. Gribaldo 2014). 
des Übersetzungsprozesses sammeln die Rechtsvertreter_innen Informationen aus den Gesprächen mit den Asylsuchenden und Expert_innen, sie benutzen Akten und Dokumente und »übertragen« sie in das rechtliche Wissenssystem, so dass sie bei den Behörden und Gerichten Gehör finden. Die Informationen werden von den Rechtsberater_innen angeordnet und in der Rechtsschrift zu einer Fallgeschichte geformt. Im Übersetzungsakt »entsteht eine Form, die vorher nicht da war« (ebd.). Es entsteht eine Fallgeschichte, die der rechtlichen Wissensdarstellung entspricht. ${ }^{16}$

Die rechtliche Kategorie legt fest, welche inhaltlichen Elemente in einer Fallgeschichte relevant sind. Während des Übersetzungsprozesses legen die Rechtsberater_innen den Fokus auf Aspekte, die von den rechtlichen Kategorien erfasst werden können. Alle anderen Aspekte der »sozialen Realitäten« werden in den Fallgeschichten irrelevant oder unerkennbar gemacht (vgl. Merry/Coutin 2014: 3). Ambiguitäten, Unsicherheiten und Verschwommenes werden ausgeblendet (vgl. Merry/Coutin 2014: 2, 3, 10, 11, 12; Yngvesson/Coutin 2006: 178). Zum Beispiel wird in Artikel 3 des Asylgesetzes (AsylG) und der damit verbundenen Rechtsprechung und Rechtslehre definiert, was in der Fallgeschichte hervorgehoben werden muss, damit abgewogen werden kann, ob eine asylsuchende Person einen Flüchtlingsstatus erhält. Sie muss laut Artikel 3 AsylG ausführen, dass sie in ihrem Heimatstaat oder im Land, in dem sie zuletzt wohnte, wegen ihrer Rasse, Religion, Nationalität, Zugehörigkeit zu einer bestimmten sozialen Gruppe oder wegen ihrer politischen Anschauung ernsthaften Nachteilen ausgesetzt war oder für ihre Zukunft begründete Furcht vor derartigen Nachteilen hat. Weiter wird im erwähnten Gesetzesartikel festgelegt, dass die Gefährdung von Leib, Leben oder Freiheit sowie Maßnahmen, die einen unerträglichen psychischen Druck bewirken, als ernsthafte Nachteile gelten. In der Rechtsprechung und -lehre werden wiederum Teilelemente des Flüchtlingsbegriffes von Artikel 3 AsylG näher festgelegt. Beispielsweise wird die Intensität der Verfolgung abgehandelt, die zu einem Flüchtlingsstatus führt oder nicht. Es wird unter anderem bestimmt, dass Folter oder direkte Angriffe auf Leib und Leben (wie Schüsse,

16 Sally Engle Merry beschreibt als Übersetzungsprozess, wie Menschenrechte einem spezifischen soziokulturellen Kontext angepasst werden und wie die Belange der lokalen Bevölkerung in den transnationalen Kontext der Menschenrechte übertragen werden (vgl. Merry 2006). Christina Giordano braucht den Begriff der Übersetzung im Zusammenhang mit institutionellen Kontexten. Sie erforscht, wie in Italien in verschiedenen institutionellen Kontexten andere Auffassungen von Staatsbürgerschaft herrschen. Jede Auffassung von Staatsbürgerschaft setzt auch eine andere Fassung der Geschichte der Migrant_innen voraus (vgl. Giordano 2008). 
Todesstrafe, Verweigerung medizinischer Hilfe, Verdurstenlassen etc.) immer als asylrelevant gelten. Ein Freiheitsentzug muss laut der Rechtsprechung und -lehre demgegenüber von einer gewissen Dauer sein, um asylbeachtliche Intensität zu erlangen (vgl. Schweizerische Flüchtlingshilfe 2009: 169f.).

In der Beschwerde für eine Person, die um den Flüchtlingsstatus ersucht, heben die Rechtsvertreter_innen deshalb in der jeweiligen Fallgeschichte die Elemente hervor, die es ermöglichen, die inhaltlichen Kriterien abzuhandeln, die in der rechtlichen Kategorie vorgegeben werden. Zum Beispiel führen sie für den individuellen Fall aus, wie ihr_e Klient_in im Gefängnis durch Schlafentzug gefoltert wurde. Alle anderen Aspekte werden in der jeweiligen Fallgeschichte nicht erwähnt und damit unsichtbar gemacht. Diese interpretative Arbeit, die die Fallgeschichte gestaltet, d.h. bestimmte Aspekte beleuchtet und andere weglässt, geht während des Übersetzungsprozesses vergessen oder wird verschwiegen. Es entstehen lineare, klare und in sich abgeschlossene Darstellungen von Migrationsgeschichten (vgl. Merry/Coutin 2014: 11f.).

Daneben bestehen auch formale Erfordernisse, die dem Recht eigen sind. Diese haben zwar nicht direkt mit der Darstellung der Fallgeschichte zu tun, dennoch sind sie von Bedeutung, damit eine Beschwerde oder ein Gesuch von den Behörden oder den Gerichten berücksichtigt wird. Zum Beispiel muss bei der Eingabe einer Beschwerde die dreißigtägige Frist eingehalten werden, andernfalls wird vom BvGer nicht auf die Beschwerde eingetreten.

Die im Übersetzungsprozess entstehende Fallgeschichte wird von den Rechtsvertreter_innen mit den Rechtsartikeln, der Rechtsprechung und anderen rechtlich relevanten Dokumenten verknüpft und verglichen (vgl. Behrends/ Park/Rottenburg 2014: 4). Das rechtliche Wissenssystem setzt voraus, welche Elemente für das Gesuch oder die Beschwerde von Bedeutung sind und wie diese angeordnet und miteinander verbunden werden. In der Rechtsschrift ordnen die Rechtsvertreter_innen zum Beispiel Informationen, die sie von ihren Klient_innen während der Konsultation erhielten, sowie Hinweise aus den Akten, die vom SEM erstellt wurden, oder Rechtsprechungen vom BvGer in neuen $\mathrm{Zu}$ sammenhängen an. Oft legen die Rechtsvertreter_innen Beweisdokumente bei, um die Fallgeschichte zu stützen. Bei einem Härtefallgesuch ${ }^{17}$ wird beispielsweise eine Bestätigung zur Sozialhilfeunabhängigkeit, ein Arbeitsvertrag und eine Sprachkursbescheinigung beigefügt. Oder eine Person, die Folter als Asylgrund geltend macht, kann einen Arztbericht zu ihren Akten legen, in dem die behandelnden Ärzt_innen eine posttraumatische Belastungsstörung diagnostizieren.

17 Ein Härtefallgesuch ist ein individuelles Gesuch um Erteilung eines Aufenthaltsstatus (siehe dazu Kapitel 4.3 dieses Beitrages). 
Zudem folgen die Argumente in einer schriftlichen Rechtseingabe meist einem Aufbau ähnlich des Syllogismus. ${ }^{18}$

Die Informationen, die in einer Fallgeschichte angeordnet werden, resultieren selbst schon aus Übersetzungsprozessen (vgl. Merry/Coutin 2014: 11). Es sind vorhergegangene, parallel verlaufende oder sich überschneidende Übersetzungsprozesse. Wie erwähnt beziehen sich die Rechtsvertreter_innen zum Beispiel auf die Anhörungsprotokolle zu den Asylmotiven ihrer Klient_innen, die das SEM erstellt hat. ${ }^{19}$ Oder sie vergleichen die Fälle ihrer Klient_innen mit bereits bestehenden Fällen aus der Rechtsprechung. Die Fallgeschichten reihen sich so in ein Geflecht von anderen Übersetzungen ein, die sich wiederum auf andere Übersetzungen beziehen. Rottenburg braucht den Begriff der »Übersetzungsketten«, um dieses Netz von »transversalen Referenten« zu beschreiben (Rottenburg 2002: 17; Merry 2006: 43).

Merry, Coutin und Wood zeigen auf, wie Recht ähnlich einem Messsystem oder einem Vergleichssystem funktioniert. Komplexe Zusammenhänge und Phänomene werden in messbare oder vergleichbare Einheiten übersetzt (vgl. Merry/Wood 2015; Merry/Coutin 2014). In diesem Sinne können Fallgeschichten als vergleichbare Einheiten angesehen werden. Durch das Formen von Fallgeschichten - und demnach von vergleichbaren Einheiten - in einem Übersetzungsprozess wird es möglich, Ähnlichkeiten und Unterschiede mit anderen Fällen aufzuzeigen und die Kriterien abzuhandeln, die in der rechtlichen Kategorie festgelegt sind (vgl. Merry/Wood 2015: 227). Die Rechtsvertreter_innen tun dies in den von ihnen verfassten Rechtsschriften und Gesuchen.

Im folgenden Kapitel zeige ich auf, wie je nach rechtlicher Kategorie drei unterschiedliche Fallgeschichten für Frau G vorausgesetzt werden. Es gelingt den Rechtsvertreter_innen in keiner der drei Fallgeschichten vollständig, die Komplexität von Frau Gs Situation in eine vergleichbare Einheit zu übersetzen, die der gewählten rechtlichen Kategorie standhält und die der rechtlichen Wissensdarstellung entspricht.

18 Ein Syllogismus besteht aus zwei Prämissen (Obersatz=Rechtsregel, Untersatz=konkreter Sachverhalt). Daraus wird eine Konklusion gezogen (vgl. Forstmoser/Vogt 2012: 614f.). Das zitierte Lehrbuch von Forstmoser und Vogt gilt als Standardwerk für die Rechtswissenschaftsstudenten an Schweizerischen Universitäten.

19 In einer breiteren Asylverwaltungsforschung untersuchte Scheffer den Prozess der Fallherstellung im Asylverfahren. Ein besonderes Augenmerk legt er dabei auf die Asylanhörungen. Er zeigt, wie die Protokolle nicht Realität darstellen, sondern Produkt eines Prozesses sind, in dem eine Vielzahl von Akteur_innen mitspielen, die von vielen Faktoren beeinflusst werden (vgl. Scheffer 2001). 


\section{Die Entstehung Von drei Fallgeschichten}

Im Hilfswerk, in dem ich Feldforschung machte, werden täglich offene Sprechstunden abgehalten. Während zwei Stunden können asylsuchende Personen ohne Voranmeldung mit eine_r Berater_in sprechen. Die Asylsuchenden, die zur Beratung kommen, wissen oft nicht genau, wie das Verfahren abläuft. Sie stellen deshalb Fragen zum Verfahrensprozess: Wie können sie bei einem negativen Entscheid Beschwerde erheben? Welche Beschwerdeinstanzen gibt es? Haben sie das Anrecht auf Familiennachzug? Wie lange dauert es, bis sie von den Behörden nach einem negativen Entscheid ausgeschafft werden? Die Rechtsberater_innen informieren ihre Klient_innen ausführlich über ihre Stellung innerhalb des Asylrechts. Sie betonten mir gegenüber immer wieder, wie wichtig es sei, dass die Asylsuchenden verstehen, welches ihre gegenwärtige und zukünftige Situation ist und welche rechtlichen Möglichkeiten sie in der Schweiz haben. Meine Kolleg_innen rieten mir zum Beispiel, das Asylsystem mit einer Skizze darzustellen, um das Verfahren zu illustrieren und für die Asylsuchenden verständlicher zu machen.

Die Rechtsberater_innen geben den asylsuchenden Personen mit diesen Informationen die Möglichkeit, ihre rechtliche Situation in der Schweiz mit den Augen der Behörden zu sehen. Den Asylsuchenden ist es dadurch eher möglich, ihre Erfolgsaussichten auf einen dauerhaften Aufenthaltstitel in der Schweiz zu vergrößern, indem sie zum Beispiel die fehlenden Dokumente beschaffen oder ihre Fluchtgründe auf eine bestimmte Art erzählen (Coutin 2003: 100, 103). Die Beratungstätigkeit dient dazu, die Fallgeschichten für die rechtlichen Gesuche oder Beschwerden im Gespräch zwischen Rechtsvertreter_in und Klient_in vorzubereiten. $^{20}$

$\mathrm{Zu}$ einer der offenen Sprechstunden kommen Frau G und ihre einjährige Tochter. $^{21}$ Frau $\mathrm{G}$ ist Mitte zwanzig und lebt seit einem knappen Jahr in der Schweiz. Vorher hat sie einige Zeit in Spanien gelebt. Dort lernte sie auch den Vater ihres Kindes kennen, von dem sie sagt, sie wisse nicht, wo er sich zurzeit aufhalte. Sie erzählt mir, sie sei im Land A geboren und im Land B aufgewach-

20 Die Vorbereitungsphasen für die rechtlichen Gesuche und Beschwerden sind unterschiedlich. Die Vorbereitungsphase bei einer Beschwerde ist kurz, meist beträgt die Frist dreißig Tage. Demgegenüber werden andere Gesuche über Jahre vorbereitet. Zum Beispiel kann es über zehn Jahre dauern, bis ein Gesuch um eine Aufenthaltsbewilligung über das Ausländerrecht eingereicht wird.

21 Alle Angaben zu den Lebensumständen von Frau G wurden anonymisiert und abgeändert. 
sen. Ich sehe Frau $G$ während fast eines halben Jahres in regelmäßigen Abständen, um mit ihr ihre rechtliche Situation in der Schweiz zu besprechen. ${ }^{22}$ Während dieser Monate ändert sich ihre Situation mehrmals. Einerseits treffen das SEM und das BvGer einen Entscheid, und später einen Zwischenentscheid, bezüglich ihres laufenden Asylverfahrens. Andererseits ändert sich auch die Lebenssituation von Frau G und sie äußert mir gegenüber andere Anliegen. Ich entdecke nach und nach weitere Aspekte ihrer Geschichte. Ich erfahre allmählich die Komplexität ihrer familiären Verhältnisse. Mit der sich verändernden Situation entstehen neue rechtliche Konstellationen. Sie verlangen nach anderen Fallgeschichten von Frau G. Indem ich Frau $G$ über einen längeren Zeitraum begleite, wird besonders deutlich, wie sich ihr Fall entwickelt. Es wird sichtbar, wie jede rechtliche Kategorie eine spezifische Fallgeschichte von derselben Person voraussetzt und wie diese Geschichten Produkte eines Prozesses sind. In den folgenden drei Unterkapiteln lege ich dar, wie die drei Darstellungen des Falles G Form annehmen. Die drei Fallgeschichten entstehen zwar chronologisch eine nach der anderen. Sie überschneiden sich aber in ihren Anfängen und Enden und laufen zeitweise auch parallel ab.

\subsection{Allgemein schwierige Lebensumstände}

Als ich Frau G kennenlerne, weiß ich schon, dass sie vom SEM einen ablehnenden Entscheid auf ihr Asylgesuch erhalten hat. Ein paar Tage zuvor brachte sie meinen Kolleg_innen ihre Asylakten. ${ }^{23}$ Sie möchte, dass die Beratungsstelle ihr hilft, eine Aufenthaltserlaubnis in der Schweiz zu bekommen. Meine Kolleg_innen beauftragten mich deshalb, ihren Fall anzuschauen und abzuklären, ob eine Chance bestehe, eine Beschwerde erfolgreich beim BvGer einzulegen.

Ich gehe im Folgenden zuerst darauf ein, wie das SEM den negativen Entscheid begründet. Indem ich zuerst diese Fallgeschichte im SEM-Entscheid beschreibe, wird später deutlicher, wie die Rechtsvertreter_innen in den weiteren Übersetzungsschritten andere rechtliche Kategorien wählen und so andere Fallgeschichten von Frau G entstehen.

22 Insgesamt sehe ich Frau G in diesem Zeitraum 16 Mal. Ihr Fall ist beim BvGer immer noch anhängig. Der Vater ihrer Tochter ist in Spanien und sie hat keinen legalen Aufenthaltsstatus in der Schweiz.

23 Die Akten von Frau G beinhalten den negativen Entscheid des SEM, die Protokolle der zwei Anhörungen, einen Auszug aus der Eurodac-Datenbank, in dem vermerkt ist, wann und wo Frau Gs Fingerabdrücke in Spanien zum ersten Mal digital erfasst wurden, und eine Kopie ihres Aufenthaltsstatus in Spanien. 
Wie ich bereits erwähnte, werden die Asylsuchenden in zwei Interviews zu ihren Asylgründen befragt. Es sind vor allem die Protokolle der zwei Anhörungen, aufgrund derer das SEM entscheidet, ob einer Person ein Aufenthaltsstatus erteilt wird oder nicht. Frau Gs zweite Anhörung durch das SEM dreht sich hauptsächlich um ihre Herkunft. In mehr als $3 / 4$ der rund 150 Fragen wird sie von der Beamt_in des SEM immer wieder zu detaillierten Angaben zu ihren Familienverhältnissen und ihrer Staatszugehörigkeit befragt. Erst am Ende des zweiten Interviews werden Fragen zu den Asylgründen gestellt. Die Beamt_in des SEM möchte wissen, welches für Frau $\mathrm{G}$ das ausschlaggebende Ereignis war, das Land B zu verlassen. Frau G beschreibt daraufhin ihre damalige Lebenssituation. Sie nennt keinen präzisen Zeitpunkt oder einzelne Begebenheiten, die sie dazu bewogen haben, wegzugehen:

»F: Was waren die Gründe dafür, dass Sie das Land B verlassen haben und in der Schweiz ein Asylgesuch stellten?

A: Bevor ich das Land verließ, lebte ich zusammen mit meiner Tante. Meine Tante hat einen Sohn. Dort wurde ich vergewaltigt. Dann hatte ich ein Missverständnis mit meiner Tante, sodass ich das Haus verlassen habe. [...] Ich hatte keine anderen Familienangehörige, darum bin ich weggegangen.

F: [...] Was war schließlich der Auslöser, dass sie das Land verlassen haben?

A: Wie ich ihnen erzählt habe, ich hatte keine Familie dort. Als ich das Haus meiner Tante verließ, war ich 15 Jahre alt. Die fünf Jahre, die ich danach verbracht habe, waren sehr schwer. Ich habe als Haushälterin gearbeitet. [...] Es gab einige Familien, die pubertierende Jungs hatten. Weil ich mich nicht wohl fühlte, wurde ich gezwungen, immer wieder meinen Arbeitsplatz zu wechseln. Das habe ich getan, weil ich nicht draußen übernachten wollte. Da dabei auch meine Moral verletzt war, konnte ich mich auch nicht auf meine Haushaltsarbeit konzentrieren. Ich war sehr traurig. Meine Freunde haben die Schule weitergemacht. Sie haben etwas erreicht. Ich habe die Schule vom 5. Schuljahr an abgebrochen. Das hat mich auch so verletzt. Darum habe ich entschlossen, das Land zu verlassen.« (Auszug Protokoll SEM)

Basierend auf den Protokollen der Anhörungen wird der negative Entscheid begründet. Der negative Entscheid ist kurz gehalten. Die Beamt_innen argumentieren in zwei Richtungen. Wie zuvor in den Fragen der Anhörung betont das SEM auch im Entscheid in einem ausführlicheren Abschnitt die Unglaubhaftigkeit der Herkunft und der Familienverhältnisse von Frau G. Das SEM wirft ihr vor, dass sie absichtlich verheimliche, dass sie eigentlich die Nationalität B besitze, zudem verschleiere sie, dass sie über ein Familien- und Bekanntennetz in Stadt C verfü- 
ge. $^{24}$ Dazu listen die Beamt_innen des SEM einige Unklarheiten bezüglich der Aussagen von Frau G im Protokoll auf. Zum anderen wird in zwei Sätzen zu ihren Asylgründen im Entscheid festgehalten, dass »die im Land B angeführte Vergewaltigung sechs Jahre vor der Ausreise aus dem Land B stattfand. [...] Es kann somit nicht mehr davon ausgegangen werden, dass dieses Ereignis in einem zeitlichen und sachlichen Zusammenhang zu ihrer Ausreise aus Land B steht.« (Auszug aus dem Entscheid des SEM). ${ }^{25}$ Frau G hat laut Entscheid keine asylrelevanten Nachteile geltend gemacht, sondern das Land verlassen, weil sie dort mit »allgemein schwierigen Lebensumständen « (Auszug aus dem Entscheid des SEM) konfrontiert war. Das SEM entscheidet deshalb, dass Frau G in das Land B zurückkehren muss.

\subsection{Verletzlichkeit}

Die Rechtsberater_innen knüpfen mit ihrem ersten Übersetzungsprozess an den oben dargelegten negativen SEM-Entscheid an. Sie haben eine Beschwerdefrist von dreißig Tagen, um auf diesen Entscheid zu reagieren. Die Beschwerde soll die Richter_innen des BvGer davon überzeugen, dass Frau G einen legalen Aufenthaltsstatus in der Schweiz erhalten sollte. Der Übersetzungsprozess, der die Fallgeschichte von Frau G so modellieren soll, dass die Beschwerde zielführend ist, findet in mehreren Situationen statt. Der Prozess erfolgt erstens in der Diskussion zwischen den Rechtsberater_innen, während der sie Erfahrungen über ähnliche Fälle austauschen. Zweitens führen die Rechtsvertreter_innen weitere Gespräche mit Frau G. Drittens werden die Asylakten von Frau G analysiert. Es sind dieselben Akten, auf die sich auch das SEM im negativen Entscheid stützt, also vor allem die Protokolle der zwei Anhörungen.

Nach einer ersten Durchsicht der Akten von Frau G suche ich in den Diskussionen mit meinen Kolleg_innen nach einer rechtlichen Kategorie, die sich am besten eignet, Frau Gs Anliegen in der Schweiz zu bleiben, zu begründen. Meine Kolleg_innen machen mich auf die Rechtsprechung des BvGer aufmerksam. Nebst einer Überzahl negativer Urteile gibt es auch einige positive Urteile zu

24 Hätte das SEM Frau G geglaubt, dass sie aus Land A ist, hätte sie nach Schweizer Asylpraxis Anrecht auf eine Aufenthaltserlaubnis in der Schweiz gehabt.

25 Laut der Rechtsprechung und -lehre den Flüchtlingsbegriff betreffend muss zwischen der Verfolgungshandlung und der Flucht »ein zeitlich und sachlicher Kausalzusammenhang stehen«. Der zeitliche Kausalzusammenhang ist unterbrochen, »wenn zwischen der erlittenen Verfolgungsmaßnahme und der Ausreise eine längere Zeit verstrichen ist« (Schweizerische Flüchtlingshilfe 2009: 187). 
ähnlichen Situationen. In diesen Urteilen gewähren die Richter_innen des BvGer der Klägerin einen Aufenthaltsstatus mit der Begründung, dass eine alleinstehende Mutter mit Kleinkind, schlechter Bildung und als Opfer sexueller Gewalt in der patriarchalen Gesellschaft des Landes B ein unwürdiges Leben führen müsse. Das Gericht ordnet deshalb an, einer solchen Frau eine vorläufige Aufnahme zu gewähren. ${ }^{26}$ Meine Kolleg_innen raten mir, die Beschwerde aufgrund dieser Rechtsprechung zu schreiben, d.h. die Fallgeschichte von Frau G mit derjenigen der Frau im Urteil zu vergleichen. Allerdings schätzen meine Kolleg_innen die Erfolgschancen der Beschwerde trotz dieser Rechtsprechung als relativ gering ein. Sie erzählen von ihren Erfahrungen mit »Land B-Fällen« und nennen zwei Anforderungen, die erfüllt sein müssen. Erstens müssen kumulative Faktoren vorhanden sein, es dürfe sich zum Beispiel nicht nur um eine junge alleinstehende Frau handeln, auch ihr Kind müsse krank sein. Zweitens führe es oft dazu, dass die gesamten Vorbringen einer asylsuchenden Person als unglaubhaft gelten, wenn das SEM an der Glaubhaftigkeit der Herkunft zweifle. Ich solle Frau G deshalb fragen, ob sie zu einem Psychiater gehe oder an anderen medizinischen Problemen leide, damit ich der Beschwerde allenfalls einen Arztbericht beilegen könnte. ${ }^{27}$

26 Eine vorläufige Aufnahme (F-Ausweis) (subsidiärer oder humanitärer Schutz) wird von den Behörden nach Art. 44 des Asylgesetzes (AsylG) erteilt, wenn der Vollzug der Wegweisung für Ausländer_innen in Situationen wie Krieg, Bürgerkrieg, allgemeiner Gewalt und medizinischer Notlage eine konkrete Gefährdung darstellt. Art. 44 AsylG bezieht sich auf das Ausländergesetz Art. 83 Abs. 4 des Ausländergesetzes (AuG). Der F-Ausweis ist auf höchstens ein Jahr befristet. In der Praxis wird er aber meistens jährlich automatisch verlängert (vgl. Schweizerische Flüchtlingshilfe 2009: 225ff.). Als Beispiel für eine alleinstehende Frau, die eine vorläufige Aufnahme erhielt, kann das Urteil vom 23. September 2010 mit der Geschäftsnummer E3430/2010 online auf der Entscheidungsdatenbank des BvGer eingesehen werden (www.bvger.ch vom 3.6.2016).

27 Gemäß Art. 44 AsylG in Kombination mit Art. 83 Abs. 4 AuG besteht eine »konkrete Gefährdung«, wenn eine weggewiesene Person eine lebensnotwendige medizinische Behandlung im Heimatstaat nicht erhalten kann. In der Praxis hat sich auch eine Kombination von Faktoren herausgebildet, wie Alter, Gesundheit, fehlendes Beziehungsnetz und schlechte Aussichten für das wirtschaftliche Auskommen als »konkrete Gefährdung«. Es wird deshalb eine vorläufige Aufnahme gewährt (F-Ausweis) (vgl. Schweizerische Flüchtlingshilfe 2009: 225ff. und das Grundsatzurteil des BvGer EMARK 1999/8). 
Die Fallgeschichte von Frau $\mathrm{G}$ wird mit Blick auf diese rechtliche Kategorie konstruiert. In mehreren Gesprächen mit Frau G versuche ich nun die zwei Anforderungen zu erfüllen, auf die mich meine Kolleg_innen aufmerksam gemacht haben, um die Chancen auf Gutheißung der Beschwerde zu erhöhen. Diese Anforderungen sind, wie bereits erwähnt, die angezweifelte Herkunft, die Familienverhältnisse und der kumulative Faktor. Bezüglich der angezweifelten Glaubhaftigkeit spreche ich Frau G auf die Ungenauigkeiten ihrer Aussagen in den Protokollen zu ihrer Herkunft an. Ich stelle ihr Fragen zu ihren Wohnorten, um chronologisch zu rekonstruieren, wo sie wann lebte. Doch die zeitliche Abfolge wird dadurch nicht klarer, eher kommen noch weitere Daten ins Spiel, die nicht die gleichen sind wie die erstgenannten Antworten. Auf mein wiederholtes Nachfragen sagt Frau G während einer unserer Gespräche, sie würde versuchen, mir vielleicht in zwei Monaten einen Geburtsschein aus Land A als Beweismittel zu besorgen. Doch sie wird dieses versprochene Dokument nie beschaffen können. In einem anderen Gespräch beginnt sie zu weinen, als ich sie abermalig auf ihre Herkunft anspreche und erkläre, wie wichtig es sei, dass sie beweisen könne, dass sie aus Land A stamme. Auf mein Drängen hin erzählt sie nochmals, dass ihre Mutter aus Land A sei, sie aber keinen Kontakt mit ihr habe.

Aufgrund der wenigen Angaben bezüglich ihrer Vergangenheit entscheide ich mich, wie es mir zuvor meine Kolleg_innen geraten haben, in der Beschwerde nicht näher auf die Herkunft und Familienverhältnisse von Frau G einzugehen. Ich argumentiere einzig im Zusammenhang mit ihrer Lebenssituation im Land B. Die Beschwerde bezieht sich auf genau die gleichen Dokumente, mit denen das SEM zuvor seinen negativen Entscheid begründet hat: Nämlich ausschließlich auf die Protokolle der Anhörung. Trotzdem ändert sich durch die von den Rechtsberater_innen gewählte rechtliche Kategorie die Perspektive. Die verschiedenen Aspekte ihres Falles werden nun anders gewichtet als im SEMEntscheid und damit eine neue Fallgeschichte produziert.

In der Beschwerde ${ }^{28}$, die in diesem Übersetzungsprozess entsteht, werden zuerst die entsprechenden Rechtsartikel und die Rechtsprechung zusammengefasst. Dann werden vier kausale Gründe aufgelistet, weshalb Frau G im Land B nicht weiterleben könne: (1) Frau G verfügt über keine gute Schul- und Berufsbildung. (2) Sie hat kein soziales Netzwerk in Stadt C. (3) Sie erlitt sexuelle und berufliche Ausbeutung als Hausmädchen. (4) Sie ist alleinstehende Mutter eines Kleinkindes. Weiter wird argumentiert, dass bei einer allfälligen Ausschaffung

28 Die Beschwerde wurde von der Autorin verfasst. Sie basiert auf einem Beschwerdemodell des Hilfswerks zu einer ähnlichen Situation wie Frau G. Die Beschwerde wurde von einem Rechtsvertreter des Hilfswerks gegengelesen. 
von Frau G sie wieder mit den gleichen Problemen konfrontiert würde, die sie zuvor zur Ausreise bewogen. Basierend auf Länderberichten wird eine patriarchale und machistische Gesellschaft im Land B beschrieben. ${ }^{29}$ Für Frau G bestehe deshalb einerseits die Gefahr der Retraumatisierung und andererseits die Gefahr, erneut Opfer sexueller Gewalt zu werden. Zusätzlich ist Frau G Mutter eines Kleinkindes, dessen Wohl durch die Rückführung mitbeeinträchtigt würde.

In der Beschwerde wird textuell eine Opfergeschichte erzählt. Es werden genau diejenigen Punkte, die vom SEM im negativen Entscheid nur oberflächlich behandelt wurden, ins Zentrum gerückt und miteinander verknüpft. Im SEMEntscheid wurde die Vergewaltigung von Frau G kaum erwähnt. Auch werden ihre »allgemein schwierigen Lebensumstände« vom SEM nicht näher beschrieben und nur in einem Satz abgehandelt. In der Beschwerde wird genau dort angesetzt und die erlittene Vergewaltigung und die Verletzlichkeit von Frau G als alleinstehende Mutter betont. Die Lage der Frauen im Land B wird im Detail beschrieben. Im Gegensatz zum SEM-Entscheid wird in der Beschwerde nicht auf die Frage ihrer Herkunft und Familienverhältnisse eingegangen. Denn wie oben beschrieben konnten in den Gesprächen mit Frau G die Unklarheiten nicht geklärt werden. In der Beschwerde legen die Rechtsberater_innen den Fokus auf die vergangene Geschichte von Frau $G$ und schließen daraus auf die Gefahren, denen sie ausgesetzt würde, wenn man sie zurückschaffte.

\subsection{Integration}

Wie ich darlegte, schätzen meine Kolleg_innen die Chancen, dass Frau G die Beschwerde gewinnen könnte, als gering ein. Sie ziehen deshalb noch eine zweite Möglichkeit in Erwägung, mit der Frau G einen Aufenthaltstitel in der Schweiz erlangen könnte: Trotz des negativen Asylentscheides kann sie als angenommene Person aus Land B nicht aus der Schweiz ausgeschafft werden. ${ }^{30}$

29 Anthony Good untersucht den Gebrauch von Herkunftsländerberichten im Asylverfahren vor Gericht. Er zeigt, wie diese Dokumente als »objektive« Beweise dienen, die die »subjektiven« Geschichten der Asylsuchenden belegen oder diese Geschichten falsifizieren (vgl. Good 2004).

30 Besteht kein Rückübernahmeabkommen zwischen der Schweiz und einem anderen Land können abgewiesene asylsuchende Personen nicht ausgeschafft werden. Je nach Herkunftsland ist es für die Schweizer Migrationsbehörden auch nicht möglich, die Ausreise der Asylsuchenden zu erzwingen, wenn die Nationalität der Gesuchsteller_innen unklar ist. Diese Herkunftsländer stellen keine Reisedokumente aus, wenn 
Frau G wird ohne Aufenthaltsbewilligung in der Schweiz bleiben können. Dies würde in ihrem Falle heißen, dass sie und ihr Kind keinen legalen Aufenthaltsstatus haben und nur von Nothilfe leben. ${ }^{31}$

Die sogenannte Härtefallregelung sieht vor, dass ein individuelles Gesuch für einen Aufenthaltsstatus (B-Ausweis) gestellt werden kann, wenn die betroffene Person sich seit der Einreichung des Asylgesuchs »seit mindestens fünf Jahren in der Schweiz aufhält«, der »Aufenthaltsort der Person den Behörden immer bekannt gewesen ist« und ein »schwerwiegender persönlicher Härtefall« vorliegt. Die Kriterien, die einen »schwerwiegenden persönlichen Härtefall« definieren, werden von einer Bundesverordnung und der bundesgerichtlichen Rechtsprechung festgelegt. Es sind dies etwa Integration, familiäre Verhältnisse (zum Beispiel der Zeitpunkt der Einschulung eines Kindes), Wille der Gesuchsteller an der Teilhabe am Wirtschaftsleben (Arbeit, keine Sozialhilfe, kein Schuldnerverzug sind vorhanden), Erwerb von Bildung (Sprache, andere Weiterbildung), Respektierung der Rechtsordnung (keine Einträge im Strafregisterauszug) und keine Möglichkeit der Wiedereingliederung in den Herkunftsstaat. ${ }^{32}$ Diese Kriterien werden je nach individueller Situation unterschiedlich gewichtet und gegeneinander abgewogen. In einem Gespräch mit meinen Kolleg_innen erzählen sie von einem ähnlichen Fall: Für eine alleinstehende Frau aus Land B, die schon seit über zehn Jahren ohne Aufenthaltsbewilligung in der Schweiz lebt, zeichnet sich jetzt endlich über die Härtefallregelung eine Lösung für einen legalen Aufenthaltsstatus ab, da sie und ihr Kind schon so lange in diesem Land lebten.

Die rechtliche Kategorie Härtefallregelung wird für Frau G im Laufe der Zeit, in der sie zur Beratungsstelle kommt, immer wichtiger. Sie wird schließlich zur einzigen Möglichkeit, mit der sie eines Tages einen geregelten Aufenthaltstitel in der Schweiz bekommen könnte. Denn etwa drei Monate nachdem die Beschwerde gegen den negativen Asylentscheid beim Gericht eingereicht wurde, erhält Frau G eine Zwischenverfügung. Das BvGer begründet in dieser Verfügung, warum es nicht auf die Beschwerde eintritt. Das Gericht folgt der gleichen Argumentation wie das SEM im negativen Entscheid für Frau G. Für die Rich-

die Nationalität der ausreisenden Personen nicht belegt ist. Dies ist zum Beispiel der Fall von Land B, in das Frau G zurückgeschafft werden sollte.

31 Abgewiesene Asylsuchende werden von der Sozialhilfe ausgeschlossen und erhalten Nothilfe. Nothilfe wird in Form von Sach- oder täglichen Geldleistungen ausgerichtet. Es wird auch eine minimale Unterkunft zur Verfügung gestellt. Abgewiesene asylsuchende Personen erhalten keine Arbeitserlaubnis und besitzen keine Ausweispapiere. Sie sind Sans-Papiers.

32 Vgl. Art. 14 AsylG und Art. 31 VZAE. 
ter_innen »[erscheinen] die Beschwerdebegehren als aussichtslos« (Auszug aus der Verfügung des BvGer). ${ }^{33}$ Ich bespreche mit Frau $G$ daraufhin die Möglichkeit der Härtefallregelung nochmals genauer. Ich mache sie darauf aufmerksam, dass es hauptsächlich darum gehe, mindestens fünf Jahre Zeit verstreichen zu lassen. ${ }^{34}$ Je länger sie warte, desto höher würden ihre Chancen auf einen Aufenthaltsstatus. Mit Schulzeugnissen und einem Sprachtest würde sie ihre Integration beweisen können. Zudem sollte sie keine Schulden machen und keine Straftaten begehen.

Im Gegensatz zur vorherigen Übersetzung spielt bei der Härtefallregelung die Vergangenheit von Frau G im Land B eine untergeordnete Rolle. Die Möglichkeit eines dauerhaften legalen Aufenthaltsstatus liegt in der Zukunft. Während in der Beschwerde die Verletzlichkeit von Frau G und ihrer Tochter in den Vordergrund gestellt wurde, werden im Ausblick auf das Härtefallgesuch ihre Eigeninitiative und ihr Wille zur Integration betont. Für das Gesuch wird die Fallgeschichte einer »erfolgreichen Integration« vorbereitet. Die Integration wird in ihrem Falle hauptsächlich an der Dauer des Aufenthalts in der Schweiz und am Schulbesuch der Tochter von Frau G gemessen.

\subsection{Familiensituation}

Die Situation von Frau $G$ ändert sich ein drittes Mal. Ein paar Monate nach der Eingabe der Beschwerde bekomme ich eine E-Mail ihrer Sozialarbeiterin. Sie informiert mich, dass der Ehemann von Frau G, Herr T, aufgetaucht sei und in der Schweiz ein Asylgesuch gestellt habe. Er sei einem anderen Kanton zugeteilt worden. Die Sozialarbeiterin will wissen, was ich tun könne, damit der Ehemann am gleichen Ort wie Frau G leben dürfe.

Mit dem Auftauchen des Ehemannes von Frau G kommt es zu einer neuen Ausgangslage. Es geht in diesem Übersetzungsprozess nicht mehr darum, eine Fallgeschichte zu formen, damit Frau G und ihre Tochter einen Aufenthaltsstatus bekommen, sondern das Zusammenleben mit ihrem Ehemann in der Schweiz wird angestrebt. Es muss deshalb eine neue rechtliche Kategorie gesucht werden, mit der das neue Anliegen von Frau G mit dem Recht vereinbar gemacht werden

33 Frau G müsste einen Kostenvorschuss zahlen, wenn sie trotzdem will, dass das Gericht auf die Beschwerde eintritt. Meine Kolleg_innen sagen mir, ich solle Frau G davon abraten, den Kostenvorschuss zu zahlen. Die Beschwerde würde sowieso vom Gericht abgelehnt werden.

34 Im Falle von Frau G wären es zum Zeitpunkt des Erhalts der Zwischenverfügung noch mindestens vier Jahre, die sie warten müsste, bis sie das Gesuch einreichen kann. 
kann. Wieder beginnt der Prozess bei der Diskussion unter den Rechtsberater_innen. Sie zeigt, dass es einige Hindernisse gibt, die einem Zusammenleben der Familie in der Schweiz im Wege stehen: Die Anwältin von Herrn T informiert mich, dass das SEM einen negativen Entscheid gefällt hat und Herrn T nach Spanien ausschaffen wird, weil er dort einen Aufenthaltsstatus besitzt. Die Anwältin erzählt weiter, dass zusätzlich die effektive Beziehung des Paares vom SEM angezweifelt wird.

Die Ausschaffung von Herrn T nach Spanien kann aus der Sicht meiner Kolleg_innen nicht verhindert werden. Frau G will nicht mit ihrem Mann zusammen nach Spanien zurück, da ihre Lebenssituation dort sehr schlecht war. Da Frau G aber keinen legalen Aufenthaltsstatus in der Schweiz hat, ist es nicht möglich, dass Herr T dank einer Familienzusammenführung in der Schweiz bleiben kann. In der Diskussion kommen wir deshalb zum Schluss, dass ich überprüfen solle, ob Herr T eine Einreisesperre für die Schweiz bekomme. Sei dies nicht der Fall, so habe er wenigstens die Möglichkeit, in die Schweiz zu Besuch zu kommen. Er könne hier zivilstandesamtlich heiraten und seine Vaterschaft offiziell anerkennen lassen. Dies jedoch erst, nachdem Frau G einen Aufenthaltsstatus in der Schweiz bekommen habe, d.h. - wie erwähnt - nach frühestens fünf Jahren und nach der Bewilligung des Härtefallgesuches. ${ }^{35}$ Besitzt Frau G einen Aufenthaltsstatus, kann sie gestützt auf die zivilstandesamtliche Heirat und die Vaterschaftsanerkennung ein Gesuch um Familiennachzug aufgrund des Ausländerrechts stellen. Meine Kolleg_innen weisen mich aber noch darauf hin, dass die Heirat schwierig werde, weil weder Frau G noch Herr T Identitätsdokumente und eine Zivilstandesbescheinigung besitzen, die in der Schweiz nötig sind, um zivilstandesamtlich heiraten zu können.

Die rechtliche Kategorie, mit der der Wunsch von Frau G nach einer Familienzusammenführung abgehandelt wird, setzt die Fallgeschichte einer Familie voraus, die in der Schweiz zivilstandesamtlich anerkannt ist. Wie vorher bei der Härtefallregelung wird auch bei dieser Übersetzung eine zukünftige Geschichte vorbereitet. In der Beschwerde war eines der Hauptargumente, dass Frau G alleinerziehende Mutter ist. Es wurde argumentiert, dass Frau G nicht wüsste, wo sich ihr Ehemann befände und sie deshalb auf sich alleine gestellt sei. Nun wer-

35 Seit Anfang 2011 müssen alle heiratswilligen ausländischen Staatsangehörigen ihren rechtmäßigen Aufenthalt in der Schweiz nachweisen. Zudem sind die Zivilstandesämter verpflichtet, die Migrationsbehörden über illegale Brautleute zu benachrichtigen. Das Bundesgericht hat jedoch entschieden, dass Sans-Papiers die Heirat in der Schweiz nicht generell verweigert werden darf. Das bedeutet, dass jeder Einzelfall geprüft werden muss. Die Praxis ist kantonal unterschiedlich. 
den ihre Heirat und die Vaterschaftsanerkennung ihrer Tochter in der Fallgeschichte besonders betont. Während bei der Härtefallregelung nicht näher auf Identitätsdokumente eingegangen wurde, muss sie in dieser Fallgeschichte darauf zurückgreifen: Die Familienzusammenführung hängt davon ab, dass Frau G Dokumente beschaffen kann, die ihre Identität und ihren Zivilstand bestätigen. Für die Familienzusammenführung ist es unwichtig, ob die Dokumente aus dem Land B oder Land A stammen. In der Beschwerde hingegen war der spezifische Länderkontext wichtig. Letztlich hängt die zukünftige Geschichte der Familienzusammenführung von der in der Zukunft liegenden Geschichte der Härtefallregelung ab. Die Familienzusammenführung wird nur möglich, wenn die Härtefallregelung angenommen wird.

\section{Schlusswort}

Fallgeschichten können als vergleichbare Einheiten eines rechtlichen Wissenssystems angesehen werden (vgl. Merry/Wood 2015; Merry/Coutin 2014). Die rechtlichen Kategorien dieses Systems bestimmen, was in einer Fallgeschichte relevant ist und welche Elemente unsichtbar gemacht werden. Am empirischen Beispiel zeigte ich, wie die Rechtsvertreter_innen drei Fallgeschichten von Frau $\mathrm{G}$ formen, um deren Anliegen zu erreichen, eine Aufenthaltserlaubnis in der Schweiz zu erhalten und um zu einem späteren Zeitpunkt auch die Familienzusammenführung zu ermöglichen. Je nach persönlicher und legaler Situation von Frau G werden von den Rechtsvertreter_innen drei verschiedene rechtliche Kategorien herangezogen. Es entstehen drei Fallgeschichten mit unterschiedlichem Fokus. Die Fallgeschichte, die der Beschwerde zugrunde liegt, fokussiert auf die Vergangenheit im Land B. In den Vordergrund tritt die Verletzlichkeit von Frau $\mathrm{G}$ und ihrem Kind. Diese Version wird schriftlich ausgeführt. In den beiden anderen Fallgeschichten werden zukünftige Szenarien entworfen, die einmal für eine konkrete Eingabe wirksam werden können. Die Rechtsvertreter_innen beraten Frau G, wie sie diese Gesuche vorbereiten kann. Da ist zum einen die mögliche Aussicht eines legalen Aufenthaltsstatus nach einer mindestens fünfjährigen Anwesenheit von Frau G und ihrem Kind als Sans-Papiers in der Schweiz. Es wird auf ihre Integration und auf die Schulkarriere ihrer Tochter gesetzt. Zum anderen wird die Geschichte einer Familienzusammenführung entworfen, die auf einer zivilstandesamtlichen Heirat mit dem Vater des Kindes beruht und die einen legalen Aufenthaltsstatus von Frau G voraussetzt.

Im empirischen Beispiel wird deutlich, wie die drei Darstellungen in einem Spannungsverhältnis zueinander stehen. Während in einer Fallgeschichte ein be- 
stimmter Aspekt betont wird und ein anderer verschwiegen, liegt in einer zweiten Version der Fokus genau auf den Aspekten, die vorher verschwiegen wurden. Es entstehen so Gegensätze zwischen den einzelnen Fallgeschichten. Zum Beispiel wird in der Beschwerde Frau $G$ als alleinstehende Frau beschrieben. In der Familienzusammenführung tritt ihre Bindung zum Vater ihres Kindes in den Vordergrund. Für das Härtefallgesuch steht das gegenwärtige Leben von Frau G in der Schweiz im Mittelpunkt, in der Beschwerde wird ihr vergangenes Leben im Land B betont. Je nach rechtlicher Kategorie werden auch die Dokumente unter einem anderen Gesichtspunkt beleuchtet. Beispielsweise sind die Identitätsdokumente sowohl für die Familienzusammenführung als auch für die Beschwerde wichtig. In der Familienzusammenführung werden die Identitätsdokumente für die zivilstandesamtliche Heirat benötigt und sollen die Identität von Frau G beweisen. In der Beschwerde sind sie wichtig, um ihre Nationalität zu belegen. Neben diesen Widersprüchen bestehen auch Abhängigkeiten zwischen den Geschichten. Die Familienzusammenführung ist nur möglich, wenn Frau G eine Aufenthaltsbewilligung erteilt würde.

Die Praktik des Übersetzens vermittelt zwischen Getrenntem und macht Elemente vergleichbar (vgl. Rottenburg 2002: 15). Doch passen im empirischen Beispiel die Fallgeschichten von Frau G nie vollständig in die jeweilige rechtliche Kategorie hinein. In allen drei Darstellungen bestehen Lücken, die nicht geschlossen werden können. In der Beschwerde fehlen die kumulativen Faktoren, die von der rechtlichen Kategorie vorausgesetzt werden, um zu befinden, dass ein Leben in Land B unmöglich sei. Auch können die Rechtsvertreter_innen die Zweifel an der Herkunft von Frau G und ihrer Familiensituation nicht beheben. Im Härtefallgesuch besteht die Schwierigkeit, dass Frau G keine Arbeitserlaubnis hat und sie so die wirtschaftliche Integration nicht erfüllen kann, um die Erfolgsaussichten des Gesuches zu erhöhen. Bei der Familienzusammenführung steht im Wege, dass weder Frau G noch ihr Ehemann Dokumente besitzen, die ihren Zivilstand bescheinigen und die für eine zivile Heirat benötigt werden. Zudem hängt die Heirat davon ab, dass Frau G einen Aufenthaltstitel besitzt. Die Rechtsvertreter_innen versuchen in den Übersetzungsprozessen diese Hindernisse kreativ zu umgehen. Indem sie andere Aspekte aus dem Fall stärker gewichten und Elemente weglassen, die nicht geklärt werden können, versuchen sie zwischen dem Getrennten zu vermitteln. Letztlich ist es ihnen aber nicht möglich, die Komplexität der Situation von Frau G in eine vergleichbare Einheit zu übersetzen, die von den rechtlichen Kategorien vorausgesetzt wird.

Am empirischen Beispiel wird sichtbar, dass sich die Rechtsberater_innen auf ein Netz von anderen vorhergegangenen oder parallel verlaufenden Übersetzungen beziehen (vgl. Rottenburg 2002; Merry/Coutin 2014). Die Rechtsvertre- 
ter_innen berufen sich vor allem auf die Protokolle der Anhörungen durch die Beamt_innen des SEM oder sie raten Frau G, ein Heiratszertifikat oder ein Vaterschaftsdokument zu beschaffen. Auch beim Härtefallgesuch werden die Integration von Frau $\mathrm{G}$ und die Schulkarriere ihrer Tochter durch ein Testresultat oder ein Schulzeugnis belegt.

Die einzelnen Fallgeschichten reihen sich in eine Kette ein, sie sind Reaktionen auf eine vorangegangene Übersetzung. Die Beschwerde entsteht als Antwort auf den negativen Entscheid des SEM. Der Übersetzungsprozess, in dem das Härtefallgesuch vorbereitet wird, wird in Gang gebracht, weil die Beschwerde von den Rechtsvertreter_innen als chancenlos eingeschätzt wird und weil das BvGer einen negativen Zwischenentscheid fällt. Die Familienzusammenführung bezieht sich auf den Aufenthaltsstatus und die zivilstandesamtliche Heirat von Frau G. Dieser Übersetzungsprozess wird initiiert, als sich die persönliche Situation von Frau $G$ verändert.

Das empirische Beispiel macht deutlich, dass die Übersetzungsprozesse in einem Umfeld ungleicher Machtverhältnisse stattfinden (vgl. Merry 2006: 40; Merry/Coutin 2014). Die Rechtsvertreter_innen übersetzen in das rechtliche Wissenssystem, dessen Kategorien spezifische Fallgeschichten voraussetzen (Coutin 2003; Merry/Coutin 2014: 3). Zwar ist es ihnen möglich, auszuwählen, welche rechtliche Kategorie sich am besten eignet, die Anliegen von Frau G zu erfüllen. Auch spielen sie kreativ mit den Aspekten, die sie in den Fallgeschichten hervorheben oder unsichtbar machen. Der Kreativität und den Strategien der Rechtsvertreter_innen sind jedoch Grenzen gesetzt. Die Rechtsvertreter_innen halten sich in allen drei Übersetzungen an die von der rechtlichen Kategorie vorausgesetzten Fallgeschichten (Verletzlichkeit, Integration, Familie). Es ist ihnen nicht möglich, die Kategorien selbst zu verändern oder außerhalb des rechtlichen Wissenssystems zu handeln, um die Anliegen von Frau G zu erfüllen. Zugespitzt formuliert können die Rechtsberater_innen, zusammen mit den Richter_innen des BvGer, den Beamt_innen im SEM oder den Mitarbeiter_innen anderer Migrationsbehörden, deshalb als »Gatekeeper_innen« angesehen werden (Yngvesson 1993: 11). Sie stehen an der Türe des rechtlichen Verfahrens und kontrollieren, wer auf welche Weise Zugang zu einer Aufenthaltsbewilligung bekommt.

\section{Literatur}

Behrends, Andrea/Park, Sung-Joon/Rottenburg, Richard (2014): »Travelling Models. Introducing an Analytical Concept to Globalisation Studies«, in: 
Dies. (Hg.), Travelling Models in African Conflict Management. Translating Technologies of Social Ordering, Leiden/Boston: Brill, S. 1-40.

Conley, John M./O'Barr, William M. (1990): Rules versus Relationships. The Ethnography of Legal Discourse, Chicago/London: University of Chicago Press.

Coutin, Susan Bibler (2003): Legalizing Moves. Salvadoran Immigrants' Struggle for U.S. Residency, Ann Arbor: University of Michigan Press.

Coutin, Susan Bibler/Mallin, Sean/Merry, Sally Engle (2014): »Technologies of Truth, Law, and Inequalities. Interview «, in: PoLAR: Political and Legal Anthropology Review Online, https://polarjournal.org/2014/06/12/interviewwith-susan-bibler-coutin-and-sally-engle-merry/ vom 3.6.2016.

D'Halluin-Mabillot, Estelle (2012): Les épreuves de l'asile. Associations et réfugiés face aux politiques du soupçon, Paris: EHESS.

DeWalt, Kathleen M./DeWalt, Billie R. (2011): Participant Observation. A Guide for Fieldworkers. Second Edition, Plymouth: AltaMira Press.

Felstiner, William L. F./Abel, Richard L./Sarat, Austin (1980): »The Emergence and Transformation of Disputes. Naming, Blaming, Claiming«, in: Law \& Society Review 15(3), S. 631-654.

Fischer, Nicolas (2009): „Une frontière négociée. L'assistance juridique associative aux étrangers placés en rétention administrative«, in: Politix 87, S. 71-92.

Forstmoser, Peter/Vogt, Hans-Ueli (2012): Einführung in das Recht. 5. Auflage, Bern: Stämpfli Verlag AG.

Giordano, Christina (2008): »Practices of translation and the making of migration subjectivities in contemporary Italy«, in: American Ethnologist 35(4), S. 588-606.

Glaser, Barney G./Strauss, Anselm L. (2010): Grounded Theory. Strategien qualitativer Forschung, Bern: Huber.

Good, Anthony (2004): »)Undoubtedly an Expert<? Anthropologists in British Asylum Courts«, in: The Journal of the Royal Anthropological Institute 10(1), S. 113-133.

Gribaldo, Alessandra (2014): »The paradoxical victim. Intimate violence narratives on trial in Italy«, in: American Anthropologist 41(4), S. 743-756.

McKinley, Michelle (1997): »Life Stories, Disclosure and the Law«, in: PoLAR. Political and Legal Anthropology Review 20(2), S. 70-82.

Merry, Sally Engle (2006): »Transnational Human Rights and Local Activism. Mapping the Middle«, in: American Anthropologist 108(1), S. 38-51.

Merry, Sally Engle/Coutin, Susan Bibler (2014): »Technologies of truth in the anthropology of conflict«, in: American Ethnologist 41(1), S. 1-16. 
Merry, Sally Engle/Wood, Summer (2015): »Quantification and the Paradox of Measurement: Translating Children's Rights in Tanzania«, in: Current Anthropology 56(2), S. 205-229.

Mertz, Elizabeth (2007): The language of law school. Learning to think slike a lawyer`, New York: Oxford University Press.

Newmahr, Staci (2008): »Becoming a Sadomasochist. Integrating Self and Other in Ethnographic Analysis« in: Journal of Contemporary Ethnography 37, S. 619-643.

Rey, Raphaël/Beurret, Olivier (2013): »>Humainement, je vous comprends; juridiquement, je ne peux rien faire $\triangleleft$. Une analyse du conseil juridique associatif dans la procédure d'asile suisse«, in: Marion Fresia/David Bozzini/Alice Sala (Hg.), Les rouages de l'asile en Suisse. Regards ethnographiques sur une procédure administrative, Neuchâtel: SFM, S. 63-93.

Rottenburg, Richard (2002): Weit hergeholte Fakten. Eine Parabel der Entwicklungshilfe, Stuttgart: Lucius \& Lucius.

Sbriccoli, Tommaso/Jacoviello, Stefano (2011): »The Case of S. Elaborating the >right $<$ narrative to fit normative/political expectations in asylum procedure in Italy«, in: Livia Hoden (Hg.), Cultural Expertise and Litigation. Patterns, Conflicts, Narratives, Abingdon: Routledge, S. 172-194.

Scheffer, Thomas (2001): Asylgewährung. Eine ethnographische Verfahrensanalyse, Stuttgart: Lucius \& Lucius.

Schweizerische Flüchtlingshilfe (Hg.) (2009): Handbuch zum Asyl- und Wegweisungsverfahren, Bern: Haupt.

Schweizerisches Bundesverwaltungsgericht, Grundsatzurteil vom 21. Januar 1999, EMARK 1999/8, www.ark-cra.ch/emark/1999/08.htm vom 3.6.2016.

Yngvesson, Barbara (1993): Virtuous Citizens, Disruptive Subjects. Order and Complaint in a New England Court, New York/London: Routledge.

Yngvesson, Barbara/Coutin, Susan Bibler (2006): »Backed by Papers. Undoing Persons, Histories and Return«, in: American Ethnologist 33(2), S. 177-190. 



\section{Auseinandersetzungen über Abschiebungen}

Handlungsoptionen in einem umkämpften Feld

\section{CARLA KÜFFNER}

»In der Küche sind zwei andere Mitarbeitende, eine Frau und ein Mann. Der Mann fragt mich nach meiner Forschung. Ich erkläre, dass ich über Verhandlungen von Rückkehr und $\mathrm{Ab}$ schiebung schreibe und mich der Aspekt der Handlungsmöglichkeiten interessiert. Da gebe es keine Handlungsmöglichkeiten, klinkt sich die Frau ein, nur untertauchen oder abgeschoben werden. Der Mann sieht das anders und sagt, bei >seinen Chinesen sei das nicht so, die bekämen die Ausreiseaufforderung, würden sie nicht verstehen und dann wegschmeißen. Sie hätten ihre Wege, seien sehr beharrlich und würden letztendlich nicht abgeschoben werden. Die Frau widerspricht vehement, sagt, seine Darstellung sei komplett falsch, argumentiert mit ihrer jahrelangen Beratungserfahrung. Zwischen den beiden entzündet sich ein hitziges Gespräch über diese Fragen.« (FORSCHUNGSTAGEBUCH JUNI 2015)

\section{Einleitung}

Die Durchführung von Abschiebungen wird in liberalen Demokratien in einer bedeutenden Anzahl von Fällen unterlassen. Trotz Ausweisungsentscheidung wird die Abschiebung aus rechtlichen oder faktischen Gründen nicht durchge- 
führt (vgl. für Österreich Welz 2014; für Deutschland Clearingstelle Trier 2011; Deutscher Bundestag 2014; für Großbritannien Paoletti 2010). Im vorliegenden Beitrag untersuche ich angeordnete Abschiebungen in Österreich ${ }^{1}$, welche zumindest zeitweilig nicht implementiert werden. Die Größenordnung dieser Fälle wird ersichtlich, wenn man den Zahlen von tatsächlichen Abschiebungen die der erlassenen Rückkehrentscheidungen ${ }^{2}$ gegenüber stellt. In diesem Beitrag zeige ich Abschiebungen ${ }^{3}$ daher als ein Feld auf, in dem sich unterschiedliche Akteur_innen in Auseinandersetzung befinden. Das Erkenntnisinteresse richtet sich auf Handlungsspielräume dieser Akteur_innen im Laufe des Abschiebeprozesses.

Das obige Zitat verdeutlicht, wie unterschiedlich die Haltungen in Auseinandersetzungen über Abschiebungen sind. Zunächst stellt sich die Frage: Können Abschiebungen verhandelt werden? Was soll es da zu verhandeln geben? Abschiebepolitik ist ein stark umkämpftes und hoch politisiertes Thema. Auch wenn es sich bei Abschiebungen zahlenmäßig um ein marginales Phänomen handelt, gibt es aktuell eine starke öffentliche Aufmerksamkeit. Gleichzeitig erstaunt es, dass über den Prozess der Abschiebung wenig bekannt ist, sodass er als Blackbox bezeichnet werden kann (vgl. Oulios 2013). Das öffentlich verfügbare Wissen ist sehr spärlich, vieles wird im Verborgenen durchgeführt. Die Uhrzeiten für den Transport zum Flughafen liegen beispielsweise meist in der Nacht, um Zuschauer_innen zu vermeiden. Die Öffentlichkeit soll abgeschirmt werden, da Abschiebungen umstritten sind. Sowohl die Abschiebehaft, wie auch die Räumlichkeiten im Flughafen, in denen sich die Personen im Abschiebegewahrsam befinden, sind Sicherheitstrakte oder Sondertransite, die für die Öffentlichkeit nicht zugänglich sind. So sind über die konkreten Abläufe während der unterschiedlichen Stationen der Abschiebung wenige Informationen verfügbar. Neben der Intransparenz ist das Feld auf behördlicher Seite von Schweigen gekennzeichnet, Forschungsanfragen werden abgelehnt (vgl. BFA 2014). Schließ-

1 Wenn nicht anders angegeben handelt es sich um Daten aus Österreich. Auf Grund der teilweise nicht vorhandenen Datenlage beziehe ich mich in einigen Teilen dieses Beitrags jedoch auch auf Deutschland.

2 Rückkehrentscheidung ist der rechtliche Ausdruck dafür, dass der legale Aufenthalt in Österreich beendet ist. Mit ihr einher geht die Aufforderung zur Ausreise. Wird dieser innerhalb einer bestimmten Frist, in der Regel einige Wochen, nicht nachgekommen, wird die Abschiebung (Außerlandesbringung) angeordnet.

3 Ich fasse hier unter dem Begriff Abschiebungen sowohl die Außerlandesbringung in den Herkunftsstaat als auch Rücküberstellungen innerhalb der EU unter der Dublin III-Verordnung. 
lich ist das Feld von rechtlicher Unsicherheit geprägt. Nach zahlreichen Änderungen im Fremdenrecht ist dieses selbst für Rechtsexpert_innen unübersichtlich geworden (vgl. Parlamentskorrespondenz 2015: o.S.).

Verglichen mit der Untersuchung der Asyl- oder Einwanderungspolitik steht die historische, politische und soziologische Analyse von Abschiebungen daher noch ganz am Anfang. Bezüglich der Frage nach Gründen und Erklärungen für die dargestellte Diskrepanz zwischen Abschiebeentscheidungen und tatsächlich implementierten Abschiebungen lassen sich in der Literatur grob drei Forschungsstränge identifizieren:

Ein erster Forschungsstrang untersucht die Frage aus der Perspektive des $\mathrm{Li}$ beralen Paradoxons unter dem Hinweis auf Schwierigkeiten, mit denen liberale Staaten bei der Implementierung von Migrationskontrollen konfrontiert werden. Liberale Demokratien, so die Argumentation, sehen sich durch selbst auferlegte Pflichten, die sich aus menschenrechtlichen Konventionen ergeben, deutlichen Einschränkungen in der Durchführung von Abschiebungen gegenüber gestellt (vgl. Bloch/Schuster 2005; Paoletti 2010). Die präsentierte Erklärung der NichtImplementierung von Abschiebungen fügt sich in ein Literaturkorpus ein, welches ein »Scheitern « von Migrationspolitiken aus dem liberalen Paradoxon heraus diskutiert (vgl. Sassen 1998; Soysal 1994). Dieser Argumentation folgend, fußen liberale Demokratien auf zwei Grundprinzipien: der Souveränität des Staates und der individuellen Freiheit. Beide Prinzipien sind sowohl konstitutiv als auch konfliktiv, denn die komplette Realisierung des einen Prinzips würde das andere aufheben und damit auch die Basis für die liberale Staatlichkeit (vgl. Mouffe 2008: 19ff.).

In einem zweiten Forschungsstrang identifizieren Autor_innen zusätzliche Gründe, die außerhalb des theoretischen Rahmens des Paradoxons stehen. Es handelt sich hierbei sowohl um technische, finanzielle als auch formale Gründe, wie bspw. fehlende Reisedokumente (vgl. Paoletti 2010; Ellermann 2008). Für mein Vorhaben von besonderem Interesse sind aus diesem Forschungsstrang die neueren Forschungen zu Widerstand und Handlungsmacht von betroffenen Migrant_innen. Mit dem Hinweis auf Anti-Abschiebungsproteste, initiiert von Geflüchteten und Migrant_innen, wird die Herausbildung eines neuen politischen Subjekts proklamiert (vgl. Ataç 2013; Isin 2009; McGregor 2011; Nyers 20103). Diese Konzeption eines neuen politischen Subjekts fügt sich in ein Literaturkorpus ein, welches innovative Forschungsperspektiven auf die Bewegung der Migration entwickelt (vgl. Forschungsgruppe TRANSIT MIGRATION 2007; Andrijasevic 2010; De Genova/Peutz 2010; Hess/Kasparek 2010). Anstatt die grenzüberschreitende Bewegung der Migration in einem Diskurs des Scheiterns von Migrationskontrollpraktiken zu verhandeln, untersuchen die Forschenden den 
Eigensinn der Migration (vgl. Benz/Schwenken 2005) eingebettet in die (System-)Bedingungen im transnationalen Raum. Solch eine Perspektive auf die Bewegung der Migration eröffnet Raum für die Analyse von Regimen, Verhandlungen, Brüchen, Widersprüchen, Handlungsmacht und Widerstandspraktiken, welche in vielen Forschungen zu Abschiebungen fehlt (vgl. Andrijasevic 2010: 149). Diese Herangehensweise entspricht dem Verständnis einer Delokalisierung von Kontrolle (vgl. Rigo 2005), welches Kontrolle nicht mehr allein an geographischen Grenzen verortet, sondern darüber hinaus innerhalb und jenseits von nationalem Raum, ausgeübt durch eine Vielzahl von (technischen) Hilfsmitteln und Akteur_innen an einer Vielzahl von Orten. So wird die Grenze als in Bewegung und flexibel beschrieben (vgl. Walters 2002) und dadurch ermöglicht, Abschiebungen als doing border von Innen zu konzeptualisieren. Auf diesem Verständnis von Grenze basiert der vorliegende Beitrag.

Ein dritter Forschungsstrang hebt die Bedeutung von Entscheidungen von Beamt_innen für die Durchführung von Abschiebungen hervor. Hier sind vornehmlich die Arbeiten der Politologin Antje Ellermann (2009) zu nennen (für Handlungsspielräume im Asylverfahren siehe auch Dahlvik 2016). Ellermann beschreibt die relative Autonomie der implementierenden Bürokratien: „On matters of immigration control, the gap between law and its implementation is colossal« (Ellermann 2009: 9).

Der Beitrag untersucht Auseinandersetzungen über Abschiebungen empirisch und ist dabei wie folgt gegliedert: Nach einer einführenden Reflexion der verwendeten Daten und Methoden wird der Ablauf des Abschiebeprozesses dargestellt. Dazu werden zunächst die unterschiedlichen Phasen erläutert und veranschaulicht, welche Akteur_innen jeweils involviert sind. Anschließend gehe ich auf Interventionsmöglichkeiten ein und untersuche so den Handlungsspielraum von ausgesuchten Akteur_innen innerhalb der unterschiedlichen Phasen im Abschiebeprozess. Im Fazit findet eine Zusammenfassung der Handlungsspielräume statt, um dann im Ausblick die Suche nach der gesellschaftlichen Funktion anzuschließen: Welche Rolle übernehmen Abschiebbarkeit (vgl. De Genova 2002) und Nichtabschiebung aus einer theoretischen Perspektive?

\section{Daten und Methoden}

Um die dargestellten Überlegungen in einem Forschungsdesign zu operationalisieren, verwende ich einen Methodenmix, der eine Rekonstruktion und Interpretation der Auseinandersetzungen ermöglicht. Es braucht Methoden, die zu fassen vermögen, wie sich die Praktiken der unterschiedlichen Akteur_innen aufeinan- 
der beziehen. Und schließlich müssen sich die Methoden für die Anwendung in einem sensiblen Untersuchungsfeld eignen. Denn die Akteur_innen von NichtAbschiebungen weisen Spezifika auf, die es bei der Forschung zu beachten gilt. Für involvierte Akteur_innen ist das (öffentliche) Sprechen bzw. Auskunft geben darüber mit einer Vielzahl an abzuwägenden Entscheidungen verbunden. Für behördlich zur Ausreise verpflichtete Personen etwa hat die Einwilligung zu einem Interview keinen positiven Einfluss auf ihren rechtlichen Status noch sonst einen direkten Nutzen. Zudem besteht vielfach die Sorge, dass die erhobenen Daten mit möglicherweise sensiblen Informationen den Behörden zugänglich gemacht werden können. Während Interviews und teilnehmende Beobachtung für Forschende in diesem Feld eine wichtige Datenquelle bedeuten, stellt sich für die Interviewpartner_innen aus den dargestellten Gründen die Frage, warum sie einwilligen sollten. Zum anderen zeigt sich, dass sich bestimmte Praktiken bzw. implizites Wissen nur eingeschränkt über Interviews erfragen lassen (vgl. Garfinkel 1967; Bergmann 2006; Breidenstein et al. 2013). Als gegenstandsangemessene Methode zur Datengenerierung in diesem sensiblen Feld bietet sich daher eine ethnographische Herangehensweise an (vgl. Forschungsgruppe TRANSIT MIGRATION 2007; Breidenstein et al. 2013). Eine zentrale methodische Besonderheit hierbei ist das Involviertwerden des/der Forschenden im Feld. Vorgesehen ist die längerfristige Begleitung der Akteur_innen, nicht ausschließlich zu Interviewzwecken, sondern auch für (informelle) Gespräche, um ein Gefühl für die Kontexte zu entwickeln, um bei Unvorhergesehenem dabei zu sein (vgl. Hess/Tsianos 2010). Dieses Verfahren ist inspiriert von George Marcus' "multi-sited ethnography« (1995). Marcus setzt Forschungsstrategien wie Tracing (Nachspüren) und Tracking (Auf-den-Fersen-bleiben) ein. Und er schlägt vor, dies nicht ausschließlich auf Personen zu beziehen, sondern den Forschungsgegenstand breiter zu fassen und auch Ideen, Bilder, Geschichten und Konflikte mit einzubeziehen.

Auf meine Forschung angewendet heißt dies, dass ich neben Interviews bzw. Gesprächen mit Akteur_innen einen Schwerpunkt auf die Kopräsenz von Beobachterin und Geschehen (vgl. Goffman 1996) lege. Dies betrifft viele unterschiedliche Orte, Behördengänge ebenso wie Beratungsgespräche bei NGOs, Besuche in Abschiebegefängnissen ebenso wie bei der Rückkehrberatung. Mein Involviertwerden geschieht über unterschiedliche Rollen, als Wissenschaftlerin, als Mitarbeiterin einer NGO, als Mitglied der Zivilgesellschaft. Diese unterschiedlichen Zugänge sind von mir gewählt worden, da das Forschungsfeld aus den oben dargestellten Gründen schwer zugänglich ist. Die Zugänge ermöglichen durch ihre Breite einerseits Einblicke in ein bisher kaum beforschtes Thema. Andererseits entstehen dadurch Herausforderungen, die mit multiplen Zuge- 
hörigkeiten und Identitätszuschreibungen im Feld einhergehen und die einer fortwährenden Reflexion bedürfen.

Die für diesen Beitrag verwendeten Daten beruhen neben fünf Interviews auf vier informellen Gesprächen, zwei Beobachtungsprotokollen, ethnographischen Feldnotizen, offiziellen Dokumenten wie Gerichtsentscheiden, Zeitungsartikeln, Statistiken des Bundesministeriums für Inneres, behördlichen Berichten und veröffentlichten Mitteilungen aus der Zivilgesellschaft. ${ }^{4}$ Für die Auswertung basierend auf Breidenstein et al. (2013) wurden zunächst die Daten dargestellt und expliziert. Daran anschließend sind Ereignisse in ihrer Funktion und Bedeutung erschlossen und analysiert und Interaktionsverläufe rekonstruiert worden.

\section{Ablauf der Abschiebung - Akteur_innen und Phasen}

Im folgenden Abschnitt werde ich die Phasen der Abschiebung identifizieren und visualisieren, welche Akteur_innen zentral sind. Dafür verdeutliche ich zunächst die Stationen bzw. Verfahrensabschnitte einer Abschiebung. In einem zweiten Schritt geht es mir darum zu zeigen, wie Abschiebungen innerhalb der verschiedenen Phasen $^{5}$ verhandelt werden. Obwohl die Kräfteverhältnisse der Akteur_innen von Ungleichheit geprägt sind, möchte ich veranschaulichen, mit welchen Praktiken auf unterschiedlichen Seiten um den Ausgang der Auseinandersetzungen über Abschiebungen gerungen wird.

In Deutschland, Österreich und anderen liberalen Demokratien findet die Durchführung von Abschiebungen in einer bedeutenden Anzahl von Fällen nicht statt (vgl. Welz 2014; Paoletti 2010). Ich frage, welche Praktiken, welche Entscheidungen und Abläufe bei diesem Phänomen zu beobachten sind und welche Handlungsspielräume sich ergeben. Dabei handelt es sich um vorläufige Befunde aus meinem Dissertationsprojekt.

Zunächst wende ich mich den Akteur_innen zu: Wer ist in die Praktiken der Abschiebung involviert? Der »Staat« scheint hier eine zentrale Rolle einzunehmen. Bei genauerer Betrachtung wird jedoch deutlich, dass vor dem Hintergrund unterschiedlicher Interessen um Kompetenzen gerungen wird und »der Staat« nicht als monolithischer Block verstanden werden kann. Stattdessen werden Widersprüche, Konflikte und Brüche sichtbar (vgl. Benz/Schwenken 2005: 4). Ne-

4 Eine Übersicht der erhobenen Daten findet sich in den Quellenangaben.

5 Bei den Phasen handelt es sich um eine schematische und vereinfachte Darstellung, die in der Praxis oft weniger differenziert sind bzw. ineinander übergehen. 
ben staatlichen Akteur_innen ist eine Vielzahl von Personen an den Auseinandersetzungen beteiligt, darunter Anwält_innen, Botschaftspersonal, Unterstützer_innengruppen, Journalist_innen, Flugpersonal, NGOs, Richter_innen usw. Schließlich zeigt sich, dass eine Akteur_innengruppe in vielen Forschungen zu Abschiebungen gar nicht als Akteur_in wahrgenommen wird, nämlich die Personen, die mit ihrer Abschiebung konfrontiert sind. Obwohl sie das zentrale Bindeglied zwischen allen involvierten Akteur_innen sind, wie auf folgender Abbildung verdeutlicht.

\section{Abbildung 1: Akteur_innen in der Aushandlung von Abschiebungen}

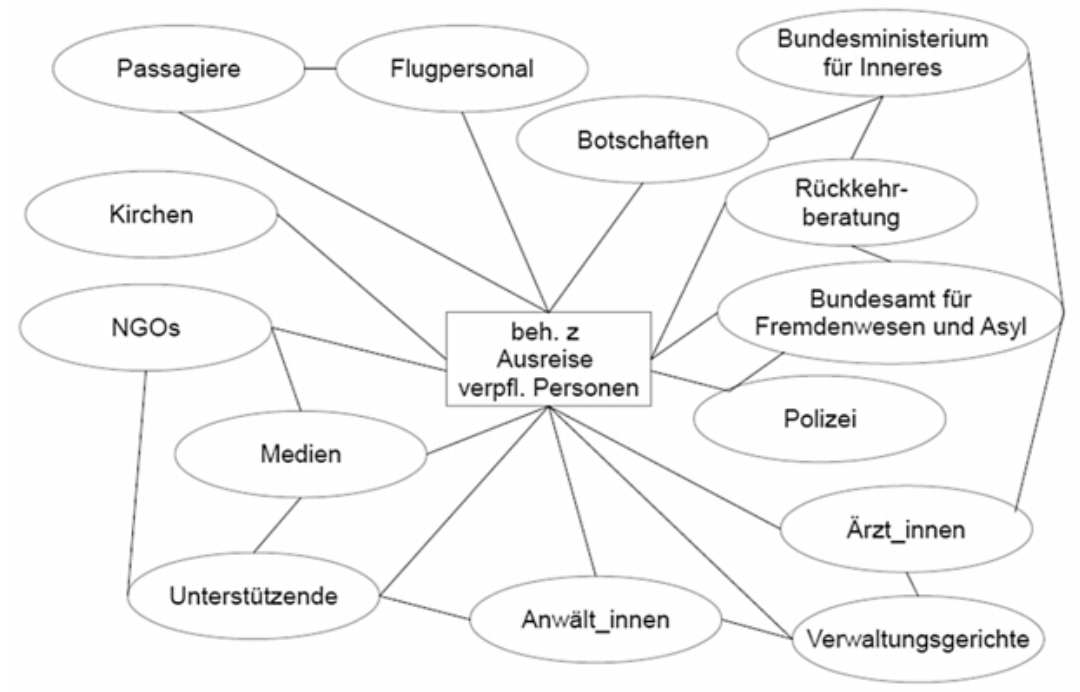

Quelle: eigene Darstellung

Die Abschiebung beginnt in Österreich für Asylbewerber_innen, die etwa die Hälfte der von Abschiebung betroffenen Personen ausmachen ${ }^{6}$, nach der Ent-

6 Die andere Hälfte der von Abschiebung betroffenen Personen setzt sich zusammen aus so genannten »Visa-overstayern«, also jenen, die legal mit einem Visum eingereist sind und die Aufenthaltsdauer überschreiten, Personen, die nicht (mehr) die für ihre Aufenthaltsbewilligung notwendigen Mindesteinkommen erzielen, die straffällig geworden sind oder deren Aufenthaltsbewilligung aus einem anderen Grund nicht verlängert wird. In diesem Beitrag fokussiere ich überwiegend Personen mit negativ abgeschlossenem Asylverfahren. 
scheidung der ersten Instanz, dem Bundesamt für Fremdenwesen und Asyl (BFA). Wird hier negativ entschieden, bedeutet dies die Ausweisung aus dem Bundesgebiet. Gegen diese Entscheidung kann innerhalb einer Frist berufen werden, dann prüft ein Verwaltungsgericht den Antrag auf Asyl. Kommt das Gericht zu einem negativen Ergebnis, wird vom BFA die Entscheidung zur Rückführung getroffen.

Darauf folgt eine Frist zur Ausreise. Die betreffende Person erhält einen Brief mit der Information, dass sie Österreich innerhalb einer gewissen Frist zu verlassen hat. Wer nach Ende dieser Frist nicht nachweislich ausgereist ist, gegen den wird die Abschiebung angeordnet. Dafür werden Reisedokumente benötigt. Liegen diese nicht vor, etwa weil sie vorher vernichtet worden sind, wird versucht, Reisezertifikate von den Botschaften der entsprechenden Herkunftsländer ausgestellt zu bekommen.

Für die Abschiebung kommt es - so noch nicht geschehen - 24-48 Stunden vor der Abschiebung zur Festnahme durch die Polizei. Mitarbeitende eines speziell geschulten Abschiebepools der Polizei führen dann die eigentliche Abschiebung durch, in den überwiegenden Fällen über den Luftweg.

Während der öffentlichen Wahrnehmung zufolge Abschiebungen mit einem spezifischen Moment in Verbindung gebracht werden, zeigt die folgende Abbildung, dass der Abschiebeprozess aus mehreren Verfahrensabschnitten besteht.

Auf dieser Abbildung sind die einzelnen Phasen gekennzeichnet, des Weiteren wird angegeben, welche Akteur_innen in welchen Verfahrensabschnitten beteiligt sind/sein können. ${ }^{7}$

7 Bei der Abbildung handelt es sich um die vereinfachte und schematische Darstellung von Verfahrensabschnitten während und nach einem Asylverfahren. Daneben gibt es weitere Gründe, warum eine Rückkehrentscheidung erlassen wird, beispielsweise weil das Einkommen unter eine bestimmte Grenze sinkt, weil eine Ehe geschieden wird, ein Visum ausläuft usw. 
Abbildung 2: Verfahrensabschnitte Abschiebeprozess

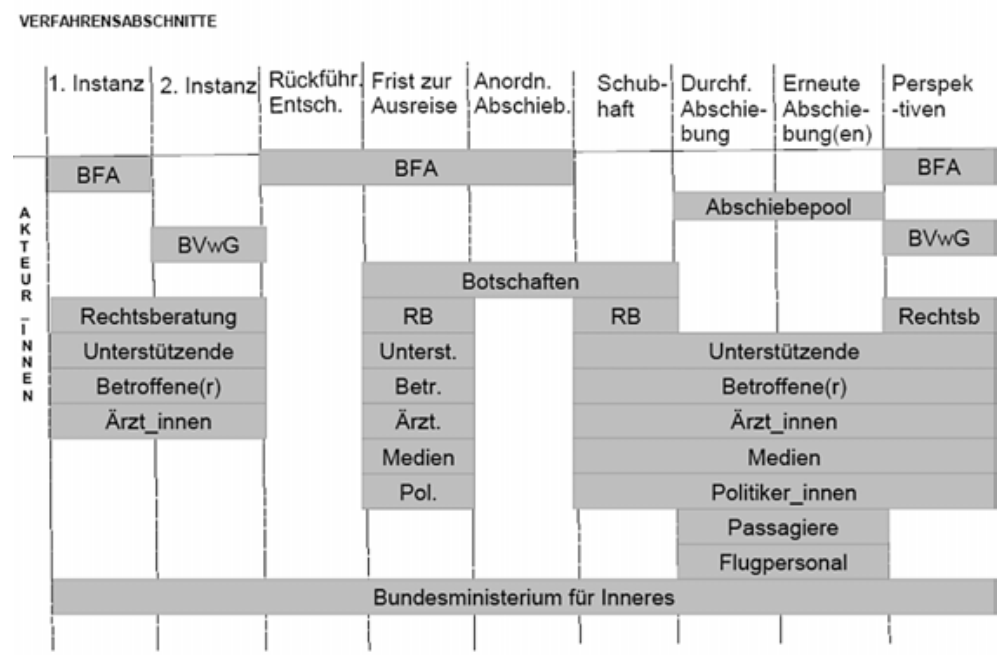

Quelle: eigene Darstellung

\section{Handlungsspielräume für Interventionen in Auseinandersetzungen über Abschiebungen}

Um Handlungsspielräume für Interventionen zu illustrieren, die Akteur_innen für eine Aushandlung von Nichtabschiebung zu eröffnen suchen, gehe ich auf ausgewählte Phasen im Abschiebeprozess näher ein.

\subsection{Berufung vor Gericht}

Eine gesetzlich vorgesehene Handlungsoption ist die Berufung vor Gericht. Entscheidet das BFA im Asylverfahren negativ, so kann vor dem Bundesverwaltungsgericht Widerspruch gegen die Entscheidung eingelegt werden. In etwa einem Fünftel der Fälle kommt das Gericht entgegen der Auffassung des BFA zu einem positiven Ergebnis (vgl. BMI 2013: 18). Das bedeutet, dass die Ausweisung aus der ersten Instanz von der zweiten Instanz aufgehoben wird und somit 
keine Abschiebung stattfindet. ${ }^{8}$ Ohne Berufung vor Gericht ist die Ausreisepflicht jedoch in der Regel 14 Tage nach dem negativen Asylbescheid rechtskräftig und die Abschiebung - so keine Ausreise erfolgt - entsprechend vollstreckbar.

\subsection{Die Frist zur Ausreise}

Die Frist zur freiwilligen Ausreise, meist beträgt sie drei Wochen, kann aus Kulanz verlängert werden, etwa um Kindern die Vollendung des Schuljahres zu ermöglichen. Ein anderes Beispiel sind Abschiebungen in bestimmte Regionen wie Serbien oder Tschetschenien, die vom Winter auf den Frühling verlegt werden.

Des Weiteren führt eine ärztlich attestierte Fluguntauglichkeit in der Regel dazu, dass eine Abschiebung nicht durchgeführt wird, wie das folgende Interviewzitat verdeutlicht. Darin erläutern zwei leitende Angestellte einer großen NGO in diesem Bereich den Erfolg von Interventionen gegen Abschiebungen. Ihrer Ansicht nach hängt dieser von der Dramatik der Geschichte ab. Sie erläutern ihre Einschätzung am Beispiel der Fluguntauglichkeit:

»I.1: Naja, über all dem steht natürlich, dass man, dass man die Person, wo man interveniert, überzeugen kann, dass tatsächlich ein Fehler passiert ist.

C.K.: Und wie kann man das machen?

I.1: Ja, indem man den Fall so präsentiert, und nachweisen kann, dass da was übersehen wurde, von wem auch immer, ja. Und dass man sich das noch einmal anschauen muss, oder, dass das halt nicht vertretbar ist, oder nicht GEHT, dass man die Person jetzt außer Landes schafft. [...] Also, eine Geschichte, die heuer ganz prominent passiert war, war die Sache mit dem Mädchen, also der jungen Erwachsenen mit der Niereninsuffizienz wo die Entscheiderin im Dublinverfahren sie tatsächlich nach Polen abschieben wollte, und das AKH [Krankenhaus in Wien] bereits eine Lebendspende der Niere von der Mutter auf die Tochter eingeleitet hatte, die Untersuchungen vorbereitet hat, und klar war, dass eine Lebendspende in Polen nicht möglich sein wird und das Mädchen lebenslänglich auf Dialyse angewiesen sein würde, und die Entscheiderin die Ansicht vertreten hat, sie hat rechtlich keine Handhabe, das Verfahren in Österreich durchzuführen. [...] Da ist es relativ leicht dann, Vorgesetzte zu überzeugen.« (iii: 2014)

8 Auch wenn mit diesen Gerichtsentscheidungen nicht in allen Fällen ein direkter Aufenthaltstitel verbunden ist, so können sich daraus langfristig Möglichkeiten für ein Bleiberecht ergeben, z.B. nach mehrjährigem legalem Aufenthalt. 
Hier wird deutlich, dass es innerhalb des rechtlichen Rahmens einen Ermessensspielraum gibt, in diesem Fall das Selbsteintrittsrecht Österreichs (Ermessensklausel Art. 17 Dublin-III Verordnung). Damit haben Behörden die Möglichkeit, eine bereits angeordnete Abschiebung innerhalb Europas zu stoppen und das inhaltliche Asylverfahren in Österreich zuzulassen. Durch die hierarchische Struktur der Behörde können sich die Rechtsberatenden an Vorgesetzte wenden, die »relativ leicht« zu überzeugen seien, wenn wie in diesem Fall eine Abschiebung nicht vertretbar ist, weil ein »Fehler«, wie es im Interview genannt wird, nachgewiesen werden kann. In diesem Beispiel wird die Situation von den Interviewten in einer Weise dargestellt, in der die Rechtsberatenden die Handelnden sind. Sie präsentieren den Fall so, dass es einen Fehler gab, und sie sind es auch, die die Vorgesetzte der Entscheiderin überzeugt haben. Die Person, die abgeschoben werden sollte und ihre Mutter kommen in diesem Narrativ nur am Rande und lediglich passiv vor. Im Interview wird nicht darüber gesprochen, wie es überhaupt dazu kam, dass die NGO sich des Falls angenommen hat, wie er an sie herangetragen wurde, und welche Hürden es möglicherweise für Mutter und Tochter zu überwinden gab, um von der NGO gehört zu werden. Die Geschichte der Fluguntauglichkeit lässt sich jedoch auch aus einer anderen, handlungsmächtigen Perspektive von Mutter und Tochter lesen ${ }^{9}$, die sich an die entsprechende NGO wandten und durch ihren Druck erreicht haben, dass mittels der Rechtsberatenden das Selbsteintrittsrecht Österreichs in Anspruch genommen wurde.

Laut den beiden interviewten NGO-Vertretern sei die Möglichkeit zur Intervention bei Vorgesetzten in der zweiten Instanz, in der das Bundesverwaltungsgericht entscheidet, deutlich eingeschränkt:

»Weil, wenn ich in einem ministeriellen System wie das BFA drinnen hänge, wo es eine klare ministerielle Hierarchie gibt, interveniere [...], in dem Fall, dann ist die Intervention an sehr hoher Stelle meistens die erfolgversprechendste. Und dann reagiert das System, wo Hierarchien vorgesehen sind, natürlich völlig anders, wie wenn ich jetzt versuche, den Präsidenten eines Gerichts anzurufen, das aus unabhängigen, weisungsungebundenen Richtern besteht. Außer, außer ein freundliches Gespräch mit dem Richter kann mir der Präsident nicht viel zusagen [...]. Das ist beim BFA, also in einer hierarchischen Organisation, wie dem, wie dem BFA, natürlich völlig anders. Die können im Notfall das anweisen. Haben sie auch schon getan.« (iii: 2014)

9 Diese Lesart speist sich aus der theoretischen Perspektive, die ich im Ausblick darlege. 
Sie beschreiben, dass sich die Intervention beim Bundesverwaltungsgericht durch die Unabhängigkeit der Richter_innen und die dadurch nicht vorhandene ministerielle Hierarchie deutlich vom Vorgehen unterscheidet, welches sie beim BFA anwenden. Die Interventionsmöglichkeiten über Vorgesetzte seien bei weisungsungebundenen Richter_innen eingeschränkt.

Die Auseinandersetzung mit rechtlichen Mitteln manifestiert sich auch in der erneuten Asylantragstellung. Hierdurch kann es in einzelnen Fällen letztendlich zu einer positiven Entscheidung kommen, wie etwa bei einer Familie, die nach ihrem fünften Antrag Asyl gewährt bekam (vgl. Bundesverwaltungsgericht 2015). In der Begründung des Gerichts heißt es:

»In einer Gesamtschau betrachtet, traten im Verfahren über den Antrag auf internationalen Schutz der Beschwerdeführer keine wesentlichen Widersprüche zu Tage, die das Vorbringen des Erstbeschwerdeführers ins Wanken hätten bringen können, sondern hat der Erstbeschwerdeführer die Geschehnisse in der mündlichen Verhandlung zusammenhängend und chronologisch geschildert und damit ein objektives und nachvollziehbares Bild seines Vorbringens vermittelt. [...] Das Ergebnis wird zudem durch die von den Beschwerdeführern vorgelegten Ladungen gestützt. [...] Auch vor dem Hintergrund der zitierten Länderberichte erscheint es nicht unplausibel, dass der Erstbeschwerdeführer [...] ins Fadenkreuz der pro-russischen Kräfte gelangt ist und ihm die Unterstützung des tschetschenischen Widerstandes zumindest unterstellt wurde.« (Bundesverwaltungsgericht 2015)

Die Feststellung, dass im Verfahren »keine wesentlichen Widersprüche« zu Tage getreten seien, wurde von den vier vorherigen Entscheider_innen im Antragsverfahren nicht geteilt. Dass es letztendlich zu einer positiven Asylentscheidung kam, steht wohl auch in Verbindung mit der Beharrlichkeit der Familie, die bereits mehrmals ausreisepflichtig war, dieser Pflicht jedoch nicht gefolgt ist.

Daneben können lokale Medien als Verhandlungsressource genutzt werden, wie der interne Behördenbericht »Vollzugsdefizite - Bericht über die Probleme bei der praktischen Umsetzung von ausländerbehördlichen Ausreiseaufforderungen« für Deutschland feststellt:

»Als gesellschaftspolitische Entwicklungen ist zu verzeichnen, dass man sich sowohl im Einzelfall als auch gruppenbezogen immer häufiger und stärker gegen die zwangsweise Beendigung der Aufenthalte ausreisepflichtiger Ausländer wendet. Interessierte Kreise haben es verstanden, ein funktionierendes länderübergreifendes Netzwerk aufzubauen, mit dem auf allen Ebenen in ihrem Sinne Einfluss ausgeübt wird. Sehr gute Kontakte zu Printmedien und auch zu TV-Sendern werden genutzt, um behördliches Handeln zu desavouieren und als inhuman anzuprangern. Die Berichterstattung ist vielfach tendenziös und schreckt auch vor der Verbreitung gezielter Unwahrheiten nicht zurück. Behördliche Be- 
strebungen um Klarstellung werden ignoriert. Eine objektive Berichterstattung findet nur selten statt. Von der bereits seit langem bestehenden Verpflichtung zur Ausreise nach langjährigen stets abschlägig verlaufenden und zum wiederholten Male durchgeführten verwaltungsgerichtlichen Verfahren durch alle Instanzen wird ebenso wenig berichtet, wie von der Tatsache, dass eine Aufenthaltsbeendigung zunächst vielfach an dem Verhalten des Betroffenen scheiterte. Rechtsstaatliches Verwaltungshandeln wird hier unter dem Deckmantel vermeintlicher Humanität als etwas >Anrüchiges〈 betrachtet.« (Clearingstelle Trier 2011: 4)

Gesellschaftliche und politische Rahmenbedingungen werden hier als Faktoren analysiert, durch die der Vollzug von Abschiebungen erschwert werde. Dabei wird der Presse eine wichtige Rolle zugesprochen, die durch tendenziöse und teilweise unwahre Berichterstattung die öffentliche Meinung gegen Abschiebungen aufbringe. Daneben werden auch (lokale) Politiker_innen explizit verantwortlich gemacht:

»Je nach politischer Konstellation der regierenden Parteien werden die Hürden für die Ausländerbehörden beim Vollzug oft durch interne Weisungen und entsprechende Erlassregelungen über bspw. besondere Prüfungsvorgaben höher und höher gehangen und dadurch aufenthaltsbeendende Maßnahmen immer mehr erschwert. Es ist aber nicht nur die Landes- und Bundespolitik, deren Unterstützung bei der Rückführung vielfach vermisst wird. Allgemein bekannt ist auch die Einflussnahme durch die Lokalpolitik auf kommunaler Ebene. Da gibt es den Bürgermeister oder Landrat, der wegen des drohenden Imageschadens aber auch angesichts nahender Kommunalwahlen in den Medien nicht in Verbindung mit Abschiebungen gebracht werden möchte oder aus eigener politischer Anschauung dem Abschiebungsvollzug seiner Ausländerbehörde den Rückhalt versagt. Landes- wie Kommunalpolitiker brechen eingeleitete Vollzugsmaßnahmen bei entsprechendem Druck immer wieder in letzter Minute ab, um den Aufenthalt rechtsgrundlos weiter zu dulden, wohl wissend, dass die Aufsichtsbehörden regelmäßig nicht einschreiten.« (Clearingstelle Trier 2011: 5)

Als eine weitere Herausforderung wird die Zusammenarbeit zwischen unterschiedlichen Behörden beschrieben. So werden Reisedokumente, welche für die Abschiebung benötigt werden, als Hindernis genannt (vgl. Clearingstelle Trier 2011: 6ff.; Ellermann 2008). Liegen diese nicht vor, z.B. weil sie vorher vernichtet worden sind, wird versucht, Reisezertifikate von den Botschaften der entsprechenden Herkunftsländer ausgestellt zu bekommen. Gerade in Fällen, in denen die Identität der betroffenen Person jedoch nicht eindeutig feststeht, eben weil 
das entsprechende Dokument fehlt, verweigern Botschaften bestimmter Staaten die Ausstellung von Reisedokumenten.

Die Beschaffung von Reisedokumenten stellt in der Praxis wohl die bedeutendste Hürde für die Abschiebung dar (vgl. Kukovetz 2014). Ein Beispiel beschreibt der Rechtsberater einer österreichischen NGO, in dem er verdeutlicht, wie sich behördlich zur Ausreise verpflichtete Personen zwischen den Anforderungen der Referent_innen des BFA und den Botschaften ihrer Herkunftsstaaten bewegen und die sich ergebenden Handlungsspielräume der Akteur_innen für sich zu nutzen suchen:

》Gelegentlich traut sich ein altgedienter Referent ein Akt liegen zu lassen. [...] Aus Gründen der Minimierung des Arbeitsaufwandes oder weil man ihm plausibel machen konnte, wir sind im humanitären Verfahren eh bald fertig, es ist nur eine Frage der Zeit, was soll dieser Wettlauf? [Der Referent] sagt dann: >Naja, ok gut; ich hab eh so viel zu tun. Ich lass das ganze liegen. Aber Sie wissen: [wir] müssen mal wieder eine Ladung schicken $\prec$.

Dann ist es auch die Frage, spiele ich in diesem komischen System so mit, dass er [der Referent] sich nicht provoziert fühlt. Dann geh ich halt zu Ladungen und sag: `Ja, wir waren bei der Botschaft, die haben die Bestätigung, sie prüfen das Ganzeく. [Referent:] >Alles in Ordnung, gehen Sie noch einmal hin, das kann so und so lang dauern. ২ Sie wissen ja eh, die Botschaft XY, die sind erfahrungsgemäß langsam. « Naja, na gut, bringen's mir heut in drei Monaten wieder einen Zettel, dass sie bei der Botschaft waren. $<$ Und dann geb ich halt alle drei Monate eine Selbstbestätigung von der Botschaft ab. [...] Es ist: Wie spiele ich das System tot? Kenne ich die Leute? Kann ich ihnen ein, auch ihren Vorgesetzten gegenüber, plausibles weiteres Vorgehen anbieten? Es ist ein Argument: Wenn jemand drei Ladungen nicht befolgt, ist der Referent auf der Palme. Wenn ich die Spielchen mitspiele und so ein bisschen mit einem kleinen Ding versehe, das was anderes herauskommt, das beabsichtigt war, hab auch keine Ahnung warum das dann so ist, gewinne ich die Zeit fürs andere [humanitäre] Verfahren. Es ist schon eine Frage: Spiel ich mit? Und kann das System ein bisschen manipulieren oder geh ich auf Konfrontation und erlebe dann, was sie können.« (i: 2013)

In dieser Beobachtung wird die Auseinandersetzung als »Spiel« beschrieben, dessen Regeln es zu kennen gilt, und das von Personen, die mit ihrer Abschiebung konfrontiert sind, mitgespielt werden kann. Der Rechtsberater verwendet wiederholt Ausdrücke wie »Spielchen mitspielen«, »im System mitspielen«, »System tot spielen«. Die Analogie zum unbedarften, kindlichen Spielen in einer so entscheidenden Situation wie der Abschiebung wirkt irritierend. Neben den zentralen Akteur_innen wie den mit ihrer Abschiebung konfrontierten Personen und den zuständigen Referent_innen des Bundesamtes tauchen die Vorgesetzten 
der Referent_innen, die Botschaftsangestellten, sowie der Begriff »das System« auf. Schließlich werden die humanitären Verfahren und damit indirekt die Abteilung, welche über ein Bleiberecht entscheidet, angesprochen. Durch entsprechende »Kniffe« eröffne sich laut Rechtsberater die Möglichkeit für die betroffenen Personen, trotz eines kooperativen Verhaltens in Bezug auf die Behörden, dennoch die Abschiebung zu verzögern und über ein humanitäres Parallelverfahren eventuell einen Aufenthaltstitel zu bekommen. Interessant ist der Aspekt der Zusammenarbeit, der hier deutlich wird. So gehe es primär darum, Ladungen zu folgen, Schriftstücke abzugeben, kurz, ein Verhalten an den Tag zu legen, welches Kooperation suggeriert und den Vorgesetzten gegenüber als »plausibles Vorgehen« angezeigt werden kann. Ob dies letztendlich zur Abschiebung führt oder nicht, scheint in dieser Darstellung zweitrangig.

Auch in dieser Beschreibung tauchen die ausreisepflichtigen Personen eher am Rande auf, die Aktivität wird ihren rechtlichen Vertretungen zugesprochen. Von behördlich zur Ausreise verpflichteten Personen wird in Gesprächen jedoch ebenfalls auf diese Praktik des »Spielens« hingewiesen, die auch ohne Unterstützung von Rechtsberatenden angewandt wird, jedoch in ihrer Beschreibung eine andere Konnotation bekommt. So findet sich beispielsweise auch in der Schilderung einer von mir interviewten ausreisepflichtigen Person die Analogie zum Spiel. Sie beschreibt es jedoch als ein Spiel, welches mit ihr gespielt werde: Immer wieder werde sie von der Behörde aufgefordert, das Identitätsdokument von der Botschaft zu liefern. Dort sage man, das Dokument sei bereits bei der Behörde. Wenn sie jedoch bei ihrem zuständigen Referenten nachfrage, ob die Identitätsfeststellung eingetroffen sei, sage dieser regelmäßig, er habe viel zu tun, sie solle sich gedulden, später wiederkommen. Durch das Hin-und-Her-GestoßenWerden fühle sie sich, als ob mit ihr »Fußball« gespielt werde (vi: 2014). Was auf der Seite des Rechtsberaters als »das System tot spielen« und damit als aktive Praktik beschrieben wird, erhält in der Darstellung der behördlich zur Ausreise verpflichteten Person einen weit weniger ermächtigenden Charakter: Sie beschreibt sich als der Ball, mit dem gespielt wird und damit als Objekt und nicht als Subjekt in der Situation. Insgesamt lässt sich festhalten, dass die Beweggründe in den Beispielen unterschiedlich scheinen, dass das »Mitspielen « jedoch die Folge haben kann, Abschiebehaft entgegenzuwirken und Zeit für andere Verfahren zu erhalten.

Ein letztes Beispiel, wie mit der Frist zur Ausreise umgegangen werden kann, ist medialer und politischer Protest durch Bündnisse, wie bereits im behördlichen Bericht über »Vollzugshindernisse« angeklungen: Im Vorarlberger Dorf Alberschwende wurde im Frühjahr 2015 Asylwerbern aus Syrien »Gemeindeasyl« gewährt. Gemeindeasyl ist kein juristischer Begriff, rein rechtlich 
kann es - ebenso wie Kirchenasyl - nicht gewährt werden. Faktisch jedoch fand es über Monate statt. Die Bürgermeisterin von Alberschwende wandte sich in diesem Zusammenhang mit einem Rundschreiben an die Öffentlichkeit.

»Durch unsere Aktivitäten mit Asylwerbern haben wir Einblick in die Unzulänglichkeiten des europäischen Asylsystems bekommen. Wir sind nicht mehr gewillt, uns gleichgültig den >Achselzuckern` anzuschließen. Wir Menschen an der Basis scheinen in puncto Asylpolitik weiter zu sein, als die mutlose und - in diesem Falle - unehrliche `hohe` Politik. « (Schwarzmann 2015: 1)

Bürgermeisterin und Anwohner_innen stellten sich Abschiebungen entgegen, mit dem Argument, durch zivilen Ungehorsam die »EU-Grundrechtscharta [zu] befolgen, indem [sie] staatlich angeordnete Deportationen verhindern, die zu Menschenrechtsverletzungen führen können« (ebd.). Damit zielt das Manifest der Gemeinde Alberschwende auf eine strukturelle Änderung des europäischen Asylsystems über die Einzelfälle in der Gemeinde hinaus. »Unsere Asylwerber«, wie sie im Dokument genannt werden, vor den Behörden zu schützen, sei ihre staatsbürgerliche Pflicht. Interessant ist, wie hier der Bruch von nationalem Recht mit EU-Recht legitimiert wird. Die Argumentation stützt sich darauf, dass durch die Verhinderung der Abschiebung eine Grundrechtsverletzung verhindert werde. Es handele sich somit nicht um den Bruch eines Gesetzes, sondern um eine gesetzliche Pflicht. Mit diesem öffentlichen zivilen Ungehorsam geht der Verhandlungsversuch einher, Einfluss auf die nationale Abschiebepolitik zu nehmen.

\subsection{Die Abschiebung}

Trotz Anordnung der eigentlichen Abschiebung wird diese teilweise nicht durchgeführt. Gründe dafür können z.B. Protest von Anwohner_innen bzw. anderen Unterstützenden sein, die sich der Abschiebung entgegenstellen und so den Transport zum Flughafen verhindern. Dies ist in der deutschen Stadt Osnabrück seit etwa einem Jahr der Fall. Zur Darstellung der Protesttechniken greife ich auf einen Artikel in der Zeitung Die Welt zurück, in dem der Protest in einer ambivalenten Haltung zwischen Faszination und Ablehnung wie folgt dargestellt wird:

»Es hat fast schon rituelle Züge, was in Osnabrück passiert, wenn eine Abschiebung ansteht. Pflichtschuldig informieren die Behörden den oder die Betroffenen vorab über den geplanten Termin, so will es das niedersächsische Innenministerium. Der Asylbewerber 
gibt das Datum einem Vertrauten durch, der wiederum das Osnabrücker Aktionsbündnis >No Lager`alarmiert. Eine Telefonkette, in der Hunderte von Abschiebungsgegnern hängen, läuft heiß.

Wenn schließlich die Polizei anrückt, um den Flüchtling zur Abreise zu eskortieren, stehen 50, 70, manchmal 90 Menschen zur Blockade bereit, selbst früh morgens um vier. Nach einer Weile ziehen die Polizisten, von höherer Stelle zur Zurückhaltung angewiesen, dann eben wieder ab. Es soll sogar vorkommen, behauptet `No Lager`, dass die Polizei erst gar nicht mehr auftaucht. Der Boykott ist dann reine Formsache.

Unter Abschiebungsgegnern bundesweit gilt Osnabrück mittlerweile als Mekka. Nirgendwo sonst wird so erfolgreich Abschiebung boykottiert. Die Aktivisten bekommen Besuche von außerhalb oder werden zu Vorträgen eingeladen. Und im Internet verkünden sie triumphierend ihre Einsätze wie Treffer in der Torschützenliste. >37. Abschiebung in Osnabrück verhindert!〈, lautete die jüngste Meldung von Mitte Juli.« (Die Welt 2015: o.S.)

Die Osnabrücker No Lager Gruppe hat gemeinsam mit ausreisepflichtigen Personen und einem breiten Bündnis aus Anwohner_innen, Kirchen etc. ein funktionierendes Netzwerk für zivilen Ungehorsam organisiert. Dabei wenden sich die Beteiligten auch direkt an die Landespolitiker_innen und fordern die Einhaltung öffentlicher Versprechen im Wahlkampf, wie etwa die vorherige Ankündigung der Abschiebung, die für das Funktionieren des Protestes zentral ist (vgl. No Lager 2015).

Konnte eine Abschiebung aus den oben dargestellten Gründen nicht durchgeführt werden, so können weitere Abschiebungen bzw. Abschiebeversuche angesetzt werden. Für innereuropäische Abschiebungen unter der Dublin IIIVerordnung gilt für die Überstellung jedoch eine 6-Monatsfrist. Dauert die Überstellung nach Zusage des Mitgliedsstaats der EU mehr als sechs Monate, so ist das inhaltliche Asylverfahren in dem Staat durchzuführen, der die fristgerechte Überstellung versäumt hat. In diesen Fällen kann eine verhinderte Abschiebung daher zur Zulassung zum Asylverfahren führen (vgl. viii: 2014).

Darüber hinaus nennen unterschiedliche Quellen mangelnde Ressourcen als weiteren Grund für die Nicht-Implementierung von Abschiebungen. Dies bezieht sich sowohl auf personelle (vgl. Clearingstelle Trier 2011: 7; Die Welt 2015: o.S.) als auch auf finanzielle Möglichkeiten (vgl. Paoletti 2010: 16).

Außerdem entzieht sich ein Teil der ausreisepflichtigen Personen der Abschiebung, indem sie sich nicht an der Adresse aufhalten, an der sie gemeldet sind. Sie tauchen unter bzw. ziehen in ein anderes Land weiter. Dies wird behördlich als »klassische Form der Vollzugsvereitelung« beschrieben und betraf in Deutschland im Jahre 2010 etwa 16\% der ausreisepflichtigen Personen (vgl. 
Clearingstelle Trier 2011: 16). Für Österreich sind keine vergleichbaren Daten veröffentlicht.

Aber selbst wenn die Person, die von Abschiebung betroffen ist, ununterbrochen an der Adresse lebt, an der sie gemeldet ist, erfolgt eine Abschiebung nicht zwingend (vgl. vi: 2014; vii: 2014; x: 2014). So lebt eine interviewte behördlich zur Ausreise verpflichtete Person seit zehn Jahren in einer Stadt in Österreich, ist dort behördlich gemeldet und wurde in diesem Zeitraum zwei Mal von den Behörden kontrolliert. Obwohl sie keinen legalen Aufenthaltsstatus hat, kam es beide Male nicht zu einer Festnahme. In diesen Fällen wissen die Behörden von der Existenz der ausreisepflichtigen Person, kennen die Adresse. Dennoch wird die Abschiebung nicht durchgeführt. Als eine zentrale Erklärung können fehlende Passersatzpapiere angenommen worden, wie weiter oben dargestellt.

Und auch Betroffene selbst stellen sich aktiv ihrer Abschiebung entgegen. Dieser Widerstand findet zu einem Zeitpunkt statt, an dem juristische Interventionen in der Regel nicht mehr möglich sind. Die Handlungsoptionen sind somit eingeschränkt, wie folgende Erzählung dokumentiert: Nach einem abgelehnten Asylantrag stellte ein Mann, ich nenne ihn hier Herr K., im Februar 2012 einen Antrag für ein Visum in Österreich, um bei Frau und Kind bleiben zu können. Als er im Mai 2012 nachts aus seiner Wohnung abgeholt wird, um in die Türkei abgeschoben zu werden ist klar, dass der Visumsantrag erfolglos geblieben ist. Herrn K. werden seine persönlichen Dinge abgenommen, seit seiner Festnahme ist ihm das Telefonieren untersagt. Bis zum Abflug sind es noch 30 Stunden. Er wird zum Flughafen gefahren. Hier wendet er sich an die Polizisten, die ihn begleiten und erklärt, dass er noch einen Haustürschlüssel bei sich hätte, den er notwendigerweise zurückgeben müsse und bittet darum, seine Frau anrufen zu dürfen. So erreicht Herr K. seine Frau am Flughafen, wohin sie sich aufgemacht hat, um gegen seine Abschiebung zu protestieren. Dort haben sich mehrere Menschen versammelt, um die Passagier_innen über die geplanten Abschiebungen zu informieren, denn zur gleichen Zeit wie Herr K. soll eine zweite Abschiebung mit einem anderen Flugzeug stattfinden.

Als Herr K. mit seiner Frau telefoniert, rät ihm diese, sich der Abschiebung zu widersetzen, indem er das Hinsetzen im Flugzeug verweigere. Zu dem Zufall der zeitgleichen zweiten Abschiebung, wegen der eine Reihe Aktivist_innen am Flughafen präsent ist, kommt der zusätzliche unwahrscheinliche Umstand, dass eine Passagierin an Bord von Herrn K.s Flugzeug mit einer Aktivistin am Flughafen befreundet ist und mit dieser in telefonischem Austausch steht. Sie kündigt dem Flugpersonal Protest gegen die Abschiebung an, informiert andere Passagier_innen und per Telefon auch die Demonstrant_innen am Flughafen. Unterdessen wird die Situation im Flugzeug vor dem Abflug immer angespannter. Um 
gegen seine bevorstehende Abschiebung zu protestieren, hält Herr K. den Passagier_innen eine Rede, in der er detailliert ausführt, welche Repressionen ihm in der Türkei drohen. Währenddessen wird er von der Stewardess mehrmals aufgefordert, seinen Platz ganz hinten im Flugzeug einzunehmen, um den Abflug zu gewährleisten. Als Reaktion verweigern drei Passagiere das Hinsetzen, viele andere schauen Herrn K. zu. Nachdem schließlich eine Passagierin die Crew auffordert, Herrn K. aus dem Flugzeug aussteigen zu lassen, erscheint der Pilot. Auf Nachfrage unterstreicht Herr K., dass die Ausreise gegen seinen Willen vollzogen wird, woraufhin der Pilot mitteilt, die Passagiere würden sich weigern, mit einem protestierenden Fluggast zu fliegen, daher dürfe die Crew ihn nicht mitnehmen. Die Abschiebung wird abgebrochen, Herr K. lebt heute mit gültigem Aufenthaltstitel gemeinsam mit seiner Frau und seinem Kind in Wien (vgl. ii: 2014; Der Standard 2012a, 2012b).

Durch die Erzählung wird deutlich, dass die Verhinderung der Abschiebung auf viele unterschiedliche Ursachen zurückgeht. Zentral ist jedoch in diesem Fall die Handlungsmacht der ausreisepflichtigen Person. So ist die Abschiebung abgebrochen worden, weil sich die behördlich zur Ausreise verpflichtete Person verbal und mit ihrem Verhalten gegen die Abschiebung zur Wehr gesetzt hat. Obwohl die Abschiebung rechtlich legal war, hat die Person dennoch mit der Illegitimität dessen argumentiert, was sie in der Türkei erwarten würde. Dadurch hat sie die Fluggäste und die Crew involviert. Damit spielt der öffentliche Raum bzw. die Öffentlichkeit eine zentrale Rolle. So weisen Bridget Anderson et al. (2011) auf Basis ihrer Untersuchung nach, dass Konflikte zwischen Bürger_innen bzw. Bürger_innen und dem Staat über die Frage entstehen können, wer Mitglied der normativen Gemeinschaft ist und damit nicht abgeschoben gehört. Im oben dargestellten Fall mischt sich dieses Argument mit Fragen der Sicherheit an Bord des Flugzeugs.

Laut Internationaler Flugtransportgesellschaft (IATA) gilt im Flugzeug die Regel, dass Crew und Pilot_in für die Sicherheit an Bord verantwortlich sind und letztendlich entscheiden, wer mitfliegt (vgl. IATA 2012: 34, 48). Eine Person, die abgeschoben wird, steht unter der Befehlsgewalt der/des Pilot_in, auch wenn sie behördlich begleitet wird. Widerstand gegen die Abschiebung kann also für die/den Pilot_in ein Grund sein, die Abschiebung abzubrechen. Die Person, die abgeschoben werden soll, verlässt das Flugzeug, das dann seine Reise aufnimmt. In der Praxis ist dies immer wieder der Fall.

Laila P., eine 30-jährige Afghanin, die sich im Juni 2015 in Wien körperlich ihrer Abschiebung widersetzte (vgl. Der Standard 2015), ist ein anderes, medienwirksames Beispiel, bei dem die Abschiebung durch den Widerstand der betroffenen Person verhindert wurde. In einer Pressemitteilung dazu heißt es unter 
der Überschrift »Geflüchtete verhindert vorerst eigene Dublin III-Abschiebung nach Bulgarien«:

»Die geplante Dublin III-Abschiebung der Geflüchteten Laila aus Afghanistan mit dem heutigen Flug von Wien nach Sofia (Bulgarien) um 7.15 Uhr konnte durch ihre couragierte Verweigerung und die Solidarität der Pilotin der Austrian Airlines, sowie des gesamten Flugpersonals verhindert werden. Laila hat beim Einstieg in das Flugzeug klar und deutlich zu verstehen gegeben, dass sie aufgrund ihrer traumatisierenden Erfahrungen und der menschenrechtswidrigen Zustände für Geflüchtete in Bulgarien nicht mitfliegen wird. Die Austrian Airlines äußerte sich bereits im Vorfeld unterstützend auf Anfrage von Freund*innen: >Grundsätzlich lehnt Austrian Airlines Abschiebungen gegen den Widerstand der Betroffenen ab. Trotz des gültigen Vertrages kann Austrian Airlines Passagiere vom Flug ausschließen, wenn [...] sie sich oder andere gefährden, oder wenn ein solcher Transport eine unzumutbare Belastung für die anderen Passagiere darstellt.« (OTS 2015)

Für Deutschland spricht der Bericht der Clearingstelle Trier davon, dass im Jahr 2009 38\% und im Jahr 2010 20\% aller »gescheiterten Rückführungen« auf den Widerstand der ausreisepflichtigen Personen zurückzuführen sind (2011: 16). Kommt es, wie in den angeführten Beispielen zu keiner Durchsetzung der Abschiebung, so können weitere Abschiebungen bzw. Abschiebeversuche angeordnet werden. Wird im Falle von innereuropäischen Abschiebungen unter der Dublin III-Verordnung die 6-Monatsfrist für Überstellungen überschritten, wird das inhaltliche Asylverfahren in dem Staat durchgeführt, der die fristgerechte Überstellung versäumt hat. Dagegen sind die Perspektiven nach einer nichtvollzogenen Abschiebung für Personen, die in ihren Herkunftsstaat abgeschoben werden sollen, deutlich eingeschränkt. Durch Integrationsnachweise kann in wenigen Fällen eine Aufenthaltsbewilligung erwirkt werden. Ein Großteil jedoch verbleibt ohne einen legalen Aufenthaltstitel oder wird bei weiteren Versuchen abgeschoben.

\section{Fazit: Von juristischen Interventionen über Aushandlungen bis zu widerständigen Körperpraktiken: die unterschiedlichen Handlungsspielräume im Abschiebeprozess}

Die empirische Analyse zeigt, dass sich die Handlungsoptionen für Interventionen im Laufe des Abschiebeprozesses verändern. Während zu Beginn die angeordnete Abschiebung über juristische Interventionen unter bestimmten Bedin- 
gungen aufgehoben werden kann, nimmt diese Möglichkeit nach der rechtskräftigen Rückführungsentscheidung deutlich ab.

Im Zuge der Frist zur Ausreise verlagern sich die Handlungsspielräume vom rechtlichen Bereich auf die Aushandlung der Art der Ausreise ${ }^{10}$, welche oftmals in einem rechtlichen Graubereich stattfindet. Wird beispielsweise nach einer durchsetzbaren Rückkehrentscheidung weiterhin die Möglichkeit einer so genannten freiwilligen Rückkehr offen gehalten, ohne die Abschiebung direkt zu vollziehen, so handelt es sich um einen Spielraum, der auf die politische Entscheidung zurückgeht, »freiwillige« Rückkehr zu priorisieren. Es wird an dieser Stelle weniger ausgehandelt, $o b$ eine Ausreise stattfindet, als vielmehr, wie. Ausnahmen bilden die politischen bzw. medialen Interventionen in Einzelfällen.

Nach der Anordnung der Abschiebung zeigt sich erneut eine Veränderung der Interventionsmöglichkeiten: Auf der einen Seite werden öffentliche Protestpraktiken eingesetzt wie etwa ziviler Ungehorsam. Auf der anderen Seite werden Praktiken im Verborgenen vollzogen, z.B. durch sich Entziehen mittels Untertauchen in die Illegalisierung. Dadurch werden Abschiebungen in einigen Fällen abgebrochen bzw. nicht durchgeführt. Die Möglichkeit, im Anschluss einen Aufenthaltsstatus zu bekommen, ist in diesem Verfahrensabschnitt im Vergleich $\mathrm{zu}$ oben jedoch sehr gering, da es sich nicht mehr um juristische Interventionen handelt.

Die Durchführung der Abschiebung lässt dann in der Regel lediglich Raum für Arten von körperlichem Widerstand. Dabei werden z.B. Praktiken bis hin zur Selbstverletzung eingesetzt, um die Flugunfähigkeit zu erreichen. Tatsächlich brechen Pilot_innen auf Grund dieses physischen Widerstandes in einer Reihe von Fällen die Abschiebung ab, indem sie sich weigern, gegen den Willen der behördlich zur Ausreise verpflichteten Person zu fliegen. Der Handlungsspielraum für behördlich zur Ausreise verpflichtete Personen ist in diesem Verfahrensabschnitt im Vergleich zu den vorherigen Phasen deutlich eingeschränkt. Oft bezieht sich der Handlungsspielraum lediglich auf den eigenen Körper.

Die vorgestellten Daten veranschaulichen, dass Aushandlungen an unterschiedlichen Punkten des Abschiebeprozesses stattfinden und dass sie sich - u.a. je nach Akteursgruppe und Verfahrensphase - deutlich unterscheiden. Abschiebungen lassen sich so nicht als einmaligen behördlichen Akt, sondern als umkämpften Prozess verstehen, an dem unterschiedliche Akteur_innen beteiligt sind.

10 Z.B. die so genannte »freiwillige Rückkehr« bzw. die Anwendung humanitärer Sonderregelungen wie das Selbsteintrittsrecht, welches die Möglichkeit beinhaltet, eine innereuropäische Abschiebung auszusetzen. 
Interessant wäre eine genauere Untersuchung, inwiefern der Ausgang der Aushandlung zusätzlich von Faktoren wie Herkunftsland, Unterstützungskreis, Familienstand, Mobilisierungspotenzial abhängt. Das »Spiel«, welches es zu kennen gilt, lässt sich möglicherweise aus einer intersektionalen Perspektive genauer beschreiben: Intersektionalität betont die Verwobenheit zwischen ungleichheitsgenerierenden Dimensionen. Dazu werden Differenzkategorien wie Geschlecht, race, Klasse, Nationalität, Alter, Religion usw. in den Blick genommen (vgl. Degele/Winkler 2007). Die Interviewten weisen in ihren Darstellungen darauf hin, dass die »Dramatik der Geschichte« eine zentrale Bedeutung hat. $\mathrm{Zu}$ untersuchen wäre daher, ob sich verschiedene Merkmale zu einer bestimmten Aushandlungsposition verweben, die sich nicht allein als Addierung von ein, zwei oder mehr ungleichheitsgenerierenden Dimensionen erklären lässt. Intersektionale Perspektiven gehen davon aus, dass diese Dimensionen in einer Weise miteinander verbunden sind, »in der sie sich wechselseitig verstärken, abschwächen oder auch verändern können« (ebd.: 1). Neben dem individuellen Verhandlungsgeschick wäre somit zu überprüfen, wie spezifische Zugehörigkeiten den Ausgang der Auseinandersetzungen beeinflussen.

Wird eine Abschiebung nicht durchgeführt, so bleibt die Abschiebbarkeit in vielen Fällen bestehen. Das zeigt, wie fragil und temporär das ausgehandelte Ergebnis ist. Jedoch steht der Ausgang der Auseinandersetzung, trotz der asymmetrischen Kräfteverhältnisse, nicht von vorneherein fest. Das Vermögen, sich seiner eigenen Abschiebung zu widersetzen und damit Widerstand zu leisten, verdeutlicht einen Aspekt von Handlungsmacht, den es genauer zu untersuchen gilt. 


\section{Ausblick: Die Suche nach der gesellschaftlichen Funktion - Abschiebbarkeit und Nichtabschiebung aus theoretischer Perspektive}

Einer gängigen Definition von Abschiebungen zufolge handelt es sich dabei um Maßnahmen, die Nicht-Staatsbürger_innen unter staatlichem Zwang außer Landes bringen (vgl. Kanstroom 2007: 29). In der Vorstellung der Migrationskontrollpolitik spielen Abschiebungen eine zentrale Rolle. Im dominanten Diskurs werden sie als Schlüsselinstrument zur Aufrechterhaltung der nationalstaatlichen Souveränität verstanden, als Möglichkeit, darüber zu entscheiden, wer sich auf dem Territorium aufhält - und wer nicht (vgl. Walters 2002). Aus dieser juristischen Perspektive handelt es sich bei Abschiebungen um das Recht von souveränen Staaten. Nichtabschiebung ist von diesem Standpunkt aus nur als Kontrolldefizit erklärbar.

William Walters (2002) weist in seinem Artikel Deportation, Expulsion, and the International Police of Aliens darüber hinaus jedoch auf eine zweite Dimension von Abschiebungen hin, den gouvernementalen Charakter. Dabei bezieht er sich auf Michel Foucaults Überlegungen zur Regierbarkeit von Bevölkerung als neue Form der Machtausübung, welche die Verwaltung und Steuerung von Personen zum Ziel hat. Mit Gouvernementalität beschreibt Foucault das Interesse politischer Macht an Wohlstand, Gesundheit, Wohlfahrt und Wachstum der Bevölkerung (vgl. Foucault 2004). Die Optimierung der Bevölkerung wird dann und so schließt sich der Kreis zur ersten Dimension - als eine Verbesserung der souveränen Macht des Staates im geopolitischen Kräfteverhältnis verstanden. Daher, so zeigt William Walters genealogisch, müssen Abschiebungen innerhalb dieses Feldes von Gouvernementalität situiert verstanden werden, nicht ausschließlich in Bezug auf Souveränität.

Es geht damit nicht um die komplette Abschottung, sondern um die Regierung der Durchlässigkeit. Bezogen auf territoriale Grenzen drückt Sandro Mezzadra die Funktion wie folgt aus:

»Es ist offensichtlich, dass ein Migrationsregime - auch wenn zu seinen eher unmittelbaren Auswirkungen die Befestigung der Grenzen und die Verfeinerung der [...] Abschiebemaschinerie gehören - nicht auf die Exklusion der Migrantinnen und Migranten zielt, sondern darauf, die Momente des Überschusses (der Autonomie) [...] zu verwerten [...].« (Mezzadra, zitiert in Tsianos/ Karakayalı 2007: 14f.) 
Was bedeutet das für das Abschieberegime ${ }^{11}$ ? Mit dem Politikwissenschaftler Peter Nyers ausgedrückt kann Abschiebepolitik nicht ausschließlich an ihrer quantitativen Effizienz gemessen werden, sondern auch daran, was durch sie transportiert wird (vgl. Nyers 2003). Wenn also nur ein Teil derer, die behördlich zur Ausreise verpflichtet sind, tatsächlich abgeschoben werden, lässt sich nach der Funktion von Abschiebungen fragen. Hier komme ich auf die Abschiebepolitik als Steuerungsinstrument zu sprechen. Für den U.S.-amerikanischen Kontext zeigt der Anthropologe Nicholas de Genova auf, wie schon die Herstellung von einem Potenzial für Abschiebungen, die Abschiebbarkeit, einen bedeutenden kontrollpolitischen Effekt hat. In seiner theoretischen Auseinandersetzung mit Abschiebbarkeit schreibt er: »What makes deportability so decisive [...] is that some are deported in order that most may remain (un-deported)« (De Genova 2002: 439). De Genova geht also davon aus, dass Abschiebbarkeit deshalb so entscheidend ist, weil Einige abgeschoben werden, damit die Meisten nichtabgeschoben bleiben. Damit benennt er einen zentralen Gedanken für ein theoretisches Verständnis von Nichtabschiebung: Für den beabsichtigten KontrollEffekt wird nicht die Abschiebung Aller benötigt. Schon die Abschiebung einiger Weniger übernimmt die Funktion, rechtliche Unsicherheit und Prekarität auch der Migrant_innen herzustellen, die bisher nicht direkt von Abschiebung betroffen waren. Personen, die um ihre Abschiebbarkeit wissen, werden sich in vielen Fällen um ihre Unsichtbarkeit bemühen, und sind dadurch leichter »regierbar«. Hier ist auch danach zu fragen, wie sich die Regierung der Durchlässigkeit auf den informellen Arbeitsmarkt auswirkt. Personen, die um ihre Abschiebbarkeit wissen, stehen dem (informellen) Arbeitsmarkt meist zu einem geringeren Lohn zu Verfügung und sind eher bereit, bestimmte unattraktive Tätigkeiten auszuüben. Anstatt also Nichtabschiebung als ein Scheitern von Kontrollpolitiken zu verstehen, schlage ich vor, die umkämpfte Durchlässigkeit in den Mittelpunkt der Analyse zu stellen.

Einen zweiten Eckpunkt bilden die individuellen Durchkreuzungsstrategien der Personen, die mit ihrer Abschiebung konfrontiert sind und darüber Ausein-

11 Der Begriff des Abschieberegimes ist geprägt durch den gleichnamigen Sammelband von Nathalie Peutz und Nicholas De Genova (2010). Mit ihm wird eine Normalisierung und Routinisierung von Abschiebungen weltweit beschrieben: »[D]espite the inevitable and irreducible historical specificities of particular states' legal bulwarks concerning the regulation of immigration (De Genova 2002), the practice of deportation has nonetheless emerged as a definite and increasingly convention of routine statecraft. Deportation seems to have become a virtually global regime.« (De Genova 2010: 34, Hervorhebung im Original) 
andersetzungen führen. Basierend auf der Perspektive der Autonomie der Migration (vgl. Forschungsgruppe TRANSIT MIGRATION 2007) wende ich dafür eine Lesart an, die Nichtabschiebung von den »Kämpfen der Migration« aus einzuordnen und zu verstehen sucht. Ziel ist es, »das umkämpfte Verhältnis zwischen staatlichem Kontrollanspruch und dem grundlegenden Recht auf Bewegungsfreiheit, das Migrant_innen sich im Zweifelsfall einfach nehmen und das diesen Kontrollanspruch daher beständig infrage stellt« (Schoenes 2013: 18), zu untersuchen. Denn die Perspektive der Autonomie der Migration geht davon aus, »dass Migration gegenüber politischen Maßnahmen, die sie zu kontrollieren beabsichtigen, ein Moment der Selbständigkeit besitzt (u.a. Moulier Boutang 2002, 2007; Mezzadra 2005, 2007, 2010).«(Schoenes 2013: 14f.)

Hat eine solche Herangehensweise Sinn? Romantisiert sie die prekären Verhältnisse von Subjekten? ${ }^{12}$ Vielleicht. »Die Herausforderung besteht mithin darin, die Handlungsmacht und die Kämpfe der Migrant_innen als Bestimmungsfaktor in die Analyse von Rückführungspolitiken einzubeziehen, ohne die objektive `Schwere` der Bedingungen zu leugnen (Mezzadra 2005: 794), unter denen diese Kämpfe stattfinden.« (Schoenes 2013: 19) Aber es gilt eben auch als die Stärke der Perspektive der Autonomie der Migration, neue Blickwinkel einzunehmen. Durch sie kann Raum für die Wahrnehmung von Widerstandspraktiken eröffnet werden, die in hegemonialen Diskursen oft unsichtbar bleiben. Mit diesem Perspektivenwechsel kann erreicht werden, die Konstruktion einer ausschließlich passiven Opferrolle der von Abschiebung betroffenen Personen aufzubrechen und ihr eine andere Geschichte entgegenzusetzen (ebd.).

Auseinandersetzungen über Abschiebungen finden in sich immer wieder verändernden Kräfteverhältnissen statt. Es geht daher um die Eröffnung einer Perspektive, die es ermöglicht, auch die Instabilitäten der Hegemonie zu fassen. Dies geht mit der Herausforderung einher, dabei gleichzeitig nicht zu vernachlässigen, dass eine Nichtabschiebung oft lediglich temporär ist und in der Regel mit keinem direkten Aufenthaltstitel einhergeht, sondern es vielmehr jederzeit (erneut) zu einer Abschiebung kommen kann.

\section{Literatur}

Anderson, Bridget/Gibney, Mathew J./Paoletti, Emanuela (2011): »Citizenship, Deportation and the Boundaries of Belonging «, in: Citizenship Studies 15(5), S. 547-563.

12 Eine ausführliche Diskussion findet sich bei Stephan Scheel (2015). 
Andrijasevic, Rutvica (2010): »From Exception to Excess: Detention and Deportations across the Mediterranean Space«, in: De Genova/Peutz, The deportation regime. Sovereignty, space, and the freedom of movement, S. 147-165.

Ataç, Ilker (2013): Die Selbstkonstituierung der Flüchtlingsbewegung als politisches Subjekt, http://eipcp.net/transversal/0313/atac/de vom 03.05.2013.

Benz, Martina/Schwenken, Helen (2005): »Jenseits von Autonomie und Kontrolle: Migration als eigensinnige Praxis«, in: Prokla 35(3), S. 363-377.

Bergmann, Jörg (2006): »Studies of Work«, in: Felix Rauner (Hg.), Handbuch der Berufsbildungsforschung, Bielefeld: Bertelsmann Verlag, S. 639-646.

Bloch, Alice/Schuster, Liza (2005): »At the extremes of exclusion: Deportation, detention and dispersal «, in: Ethnic and Racial Studies 28(3), S. 491-512.

Breidenstein, Georg/Hischauer, Stefan/Kalthoff, Herbert/Nieswand, Boris (2013): Ethnographie. Die Praxis der Feldforschung, München/Konstanz: UVK.

Bundesverwaltungsgericht 2015: Geschäftszahl W196 1405573-7.

Dahlvik, Julia (2016): »Asylanträge verwalten und entscheiden: der soziologische Blick auf Verborgenes. Eine Forschungsnotiz«, in: Österreichische Zeitschrift für Soziologie 41, S. 191-205.

Degele, Nina/Winkler, Gabriele (2007): Intersektionalität als Mehrebenenanalyse, http://portal-intersektionalitaet.de/theoriebildung/schluesseltexte/degele winker/ vom 04.08.2015.

De Genova, Nicholas (2002): »Migrant >Illegality〈 and Deportability in Everyday Life«, in: Annual Review of Anthropology 31(1), S. 419-447.

De Genova, Nicholas (2010): »The deportation regime. Sovereignty, space, and the freedom of movement«, in: De Genova/Peutz, The deportation regime. Sovereignty, space, and the freedom of movement, S. 33-65.

De Genova, Nicholas/Peutz, Nathalie Mae (Hg.) (2010): The deportation regime. Sovereignty, space, and the freedom of movement. Durham, NC: Duke University Press.

Deutscher Bundestag (2014): Antwort der Bundesregierung auf die Kleine Anfrage: Ergänzende Informationen zur Asylstatistik für das zweite Quartal 2014, Drucksache 18/2471.

Ellermann, Antje (2008): »The Limits of Unilateral Migration Control: Deportation and Interstate Cooperation«, in: Government and Opposition 43(2), S. 168-189.

Ellermann, Antje (2009): States against migrants. Deportation in Germany and the United States, Cambridge, NY: Cambridge University Press. 
Forschungsgruppe TRANSIT MIGRATION (Hg.) (2007): Turbulente Ränder. Neue Perspektiven auf Migration an den Grenzen Europas. Bielefeld: transcript.

Foucault, Michel (2004): Geschichte der Gouvernementalität. Sicherheit, Territorium, Bevölkerung: Vorlesungen am Collège de France 1977-1978, Frankfurt a.M.: Suhrkamp.

Garfinkel, Harold (1967): Studies in ethnomethodology, Englewood Cliffs, NJ: Prentice Hall.

Goffman, Erving (1996): »Über Feldforschung«, in: Hubert Knoblauch (Hg.), Kommunikative Lebenswelten. Zur Ethnographie einer geschwätzigen Gesellschaft, Konstanz: UKV, S. 261-269.

Hess, Sabine/Kasparek, Bernd (2010): Grenzregime. Diskurse, Praktiken, Institutionen in Europa, Berlin/Hamburg: Assoziation A.

Hess, Sabine/Tsianos, Vassilis (2010): »Ethnographische Grenzregimeanalysen. Eine Methodologie der Autonomie der Migration«, in: Sabine Hess/Bernd Kasparek (Hg.), Grenzregime. Diskurse, Praktiken, Institutionen in Europa, Berlin/Hamburg: Assoziation A, S. 243-264.

Isin, Engin (2009): »Citizenship in flux: The figure of the activist citizen «, in: Subjectivity 29, S. 367-388.

Kanstroom, Daniel (2007): Deportation Nation. Outsiders in American History, Cambridge/London: Harvard University Press.

Kukovetz, Brigitte (2014): »Der Wunsch zu bleiben: Kein Recht dazu - keine Alternative dazu. Ein Einblick in soziale Praktiken: Warum Abschiebungen doch nicht stattfinden«, in: Julia Dahlvik/Christoph Reinprecht/Wiebke Sievers (Hg.), Migration und Integration - wissenschaftliche Perspektiven aus Österreich. Jahrbuch 02/2013, Wien: Vienna University Press, S. 261-282.

Marcus, George (1995): »Ethnography in/of the world system: The Emergence of Multi-Sited Ethnography«, in: Annual Review of Anthropology 24, S. 95-117.

McGregor, JoAnn (2011): »Contestations and consequences of deportability: hunger strikes and the political agency of non-citizens«, in: Citizenship Studies 15(5), S. 597-611.

Mezzadra, Sandro (2005): »Der Blick der Autonomie«, in: Kölnischer Kunstverein (Hg.), Projekt Migration. Ausstellungskatalog, Köln: DuMont Verlag, S. 794-795.

Mezzadra, Sandro (2007): »Kapitalismus, Migrationen, soziale Kämpfe«, in: Pieper/Atzert/Karakayali/Tsianos, Empire und die biopolitische Wende. Die internationale Diskussion im Anschluss an Hardt und Negri, S. 179-193. 
Mezzadra, Sandro (2010): »Autonomie der Migration - Kritik und Ausblick. Eine Zwischenbilanz«, in: Grundrisse. Zeitschrift für linke Theorie \& Debatte 34, S. 22-29.

Mouffe, Chantal (2008): Das Demokratische Paradox, Wien: Turia und Kant.

Moulier Boutang, Yann (2002): »Nicht länger Reservearmee. Thesen zur Autonomie der Migration und zum notwendigen Ende des Regimes der Arbeitsmigration«, in: Subtropen, Monatliches Supplement der Jungle World 2002(4), http://jungle-world.com/artikel/2002/14/24171.html vom 6.4.2015.

Moulier Boutang, Yann (2007): »Europa, Autonomie der Migration, Biopolitik«, in: Pieper/Atzert/Karakayali/Tsianos, Empire und die biopolitische Wende. Die internationale Diskussion im Anschluss an Hardt und Negri, S. 169-178.

Nyers, Peter (2003): »Abject cosmopolitanism: the politics of protection in the anti-deportation movement«, in: Third World Quarterly 24(6), S. 1069-1093.

Oulios, Miltiadis (2013): Blackbox Abschiebung. Geschichten und Bilder von Leuten, die gerne geblieben wären, Berlin: Suhrkamp.

Paoletti, Emanuela (2010): Deportation, non-deportability and ideas of membership, Working Paper Series, no. 65, Refugee Studies Center.

Pieper, Marianne/Atzert, Thomas/Karakayali, Serhat/Tsianos, Vassilis (Hg.) (2007): Empire und die biopolitische Wende. Die internationale Diskussion im Anschluss an Hardt und Negri, Frankfurt a.M./New York: Campus.

Rigo, Enrica (2005): »Citizenship at Europe's Borders: Some Reflections on the Post-colonial Condition of Europe in the Context of EU Enlargement «, in: Citizenship Studies 9(1), S. 3-22.

Sassen, Saskia (1998): »The de facto Transnationalizing of Immigration Policy. Territory, Authority, Rights. From Medieval to Global Assemblages«, in: Joppke (Hg.), Challenge to the Nation State. Immigration in Western Europe and the United States, Oxford: Oxford University Press, S. 49-85.

Scheel, Stephan (2015): »Das Konzept der Autonomie der Migration überdenken? Yes, please!«, in: movements 2015. Journal für kritische Migrationsund Grenzregimeforschung 1(2), http://movements-journal.org/issues/02. kaempfe/14.scheel--autonomie-der-migration.html vom 05.06.2016.

Schoenes, Katharina (2013): Politiken der freiwilligen Rückführung. Eine Analyse aus der Perspektive der Migration. Masterarbeit, Berlin.

Soysal, Yasemin Nuhoğlu (1994): Limits of Citizenship. Migrants and Postnational Membership in Europe, Chicago: University of Chicago Press.

TRANSIT MIGRATION (Hrsg.) (2007): Turbulente Ränder. Neue Perspektiven auf Migration an den Grenzen Europas, Bielefeld: transcript. 
Tsianos, Vassilis/ Karakayalı, Serhat (2007): »Movements that matter. Eine Einleitung«, in: Forschungsgruppe TRANSIT MIGRATION, Turbulente Ränder. Neue Perspektiven auf Migration an den Grenzen Europas., S. 7-17.

Walters, William (2002): »Deportation, Expulsion and the International Police of Aliens«, in: Citizenship Studies Volume 6(3), S. 265-295.

Welz, Judith (2014): Die österreichische Abschiebepolitik in Zahlen. 1995-2013. INEX Working Paper Nr.1.

\section{Internetquellen}

Bundesministerium für Inneres (BMI) (2013): Asylstatistik 2013, http://www.bmi.gv.at/cms/BMI_Asylwesen/statistik/files/Asylstatistik_Jahre sstatistik_2013.pdf vom 27.07.2015.

Clearingstelle Trier (2011): Vollzugsdefizite. Ein Bericht über die Probleme bei der praktischen Umsetzung von ausländerbehördlichen Ausreiseaufforderungen, http://www.proasyl.de/fileadmin/proasyl/fm_redakteure/Newsletter_ Anhaenge/171/2011-04_Bericht_AG_Rueck.pdf vom 27.07.2015.

Der Standard (2012a): Drohende Familientrennung für politischen Aktivisten aus Türkei, http://derstandard.at/1339638916847/Abschiebung-DrohendeFamilientrennung-fuer-politischen-Aktivisten-aus-Tuerkei vom 22.12.2015.

Der Standard (2012b): Abschiebung trotz Rückkehr, http://derstandard.at/ 1342947413208/Abschiebung-trotz-Rueckkehr vom 22.12.2015.

Der Standard (2015): Fall Laila P.: Pilotencrew verhinderte umstrittene Rückschiebung, http://derstandard.at/2000017617047/Fall-Laila-P-Pilotencrewverhinderte-umstrittene-Rueckschiebung vom 04.08.2015.

Die Welt (2015): Mit diesen Tricks werden Abschiebungen verhindert, http://www.welt.de/politik/deutschland/article145799121/Mit-diesen-Trickswerden-Abschiebungen-verhindert.html vom 07.10.2015.

IATA (2012): Guidance on unruly passenger prevention and management, http://www.iata.org/whatwedo/safety/Documents/Guidance-On-UnrulyPassenger-Prevention-And-Management-1 st-Edition.pdf vom 20.06.2015.

No Lager (2015): Offener Brief an Innenminister Boris Pistorius zur Neureglung des Rückführungserlasses, http://nolageros.blogsport.eu/ vom 28.09.2015.

OTS (2015): Geflüchtete verhindert vorerst eigene Dublin III-Abschiebung nach Bulgarien, http://www.ots.at/presseaussendung/OTS_20150617_OTS0229/ gefluechtete-verhindert-vorerst-eigene-dublin-iii-abschiebung-nachbulgarien vom 21.09.2015. 
Parlamentskorrespondenz (2015): Innenausschuss stimmt Fremdenrechtsnovelle mit S-V-T-Mehrheit zu. Abänderungsantrag im Plenum könnte noch einige Nachbesserungen bringen, http://www.parlament.gv.at/PAKT/PR/JAHR 2015/PK0488/ vom 04.08.2015.

Schwarzmann, Angelika (2015): Manifest der Gemeinde Alberschwende, http:// www.vindex.or.at/manifest-der-gemeinde-alberschwende/ vom 10.07.2015.

\section{Übersicht erhobene Daten}

i: Interview Rechtsberater NGO, Dauer 60 min, Transkript.

ii: Interview mit vormals behördlich zur Ausreise verpflichteter Person, 16.11.2014, Dauer 55 min, Gedankenprotokoll.

iii: Interview Geschäftsführer und Leiter Rechtsberatung NGO, 03.12.2014, Dauer 57 min, Transkript.

iv: Interview mit Teamleiter und Jurist Rückkehrberatung NGO, 10.02.2015, Dauer 75 min, Transkript.

v: Interview Rechtsberater NGO, 24.04.2015, Dauer 43 min, Transkript.

vi: Gespräch mit behördlich zur Ausreise verpflichteter Person, 15.05.2014, 120 min, Gedankenprotokoll.

vii: Gespräch mit behördlich zur Ausreise verpflichteter Person, 27.05.2014, Dauer 40 min, Gedankenprotokoll.

viii: Gespräch mit vormals behördlich zur Ausreise verpflichteter Person, 11.09.2014, Dauer 30 min, Gedankenprotokoll.

ix: Gespräch mit Behördenvertreterin, 22.10.2014, Dauer 100 min, Gesprächsprotokoll.

x: Beobachtungsprotokoll BFA 27.05.2014

xi: Beobachtungsprotokoll Bundesverwaltungsgericht 18.06.2014.

Bundesamt für Fremdenwesen und Asyl (2014): Emailkorrespondenz 07.11.2014. 


\title{
Autorinnen und Autoren
}

\begin{abstract}
Affolter, Laura, ist Doktorandin am Institut für Sozialanthropologie der Universität Bern. Sie arbeitet dort im Forschungsprojekt »Doing credibility. The construction of credibility in Swiss asylum procedures « mit, welches vom Schweizerischen Nationalfonds gefördert wird. In ihrer Dissertation setzt sie sich mit Entscheidungspraktiken und dem institutionellen Habitus im Staatssekretariat für Migration auseinander.
\end{abstract}

Amelung, Nina, ist research fellow am Center for Social Studies (CES), Universität Coimbra, Portugal, und in der Innovation in Governance Research Group, Technische Universität Berlin. Zu ihren Forschungsschwerpunkten gehören transnationale Politikinnovationen, Demokratieforschung und Partizipationsforschung.

Dahlvik, Julia, ist promovierte Soziologin und Translationswissenschaftlerin und derzeit Projektmitarbeiterin am Institut für Stadt- und Regionalforschung der Österreichischen Akademie der Wissenschaften und Lektorin an der Universität Wien. Ihre Forschungsschwerpunkte liegen in den Bereichen Migration, Organisation, Recht und Gesellschaft.

Eule, Tobias, ist Assistenzprofessor für Rechtssoziologie an der Universität Bern und Fellow im Department of Anthropology an der London School of Economics. Zu seinen Forschungsschwerpunkten gehören die Anwendung von Migrationsrecht in staatlichen Behörden (Inside Immigration Law, Ashgate 2014), juristische Arbeitswelten (Recht als Beruf, Stämpfli 2017) und der Zugang zu Recht in prekären Lebenssituationen.

Fuchs, Johanna, ist seit Februar 2014 Doktorandin am Institut für Sozialanthropologie der Universität Bern unter Betreuung von Prof. Dr. Julia Eckert. In ih- 
rem Dissertationsprojekt setzt sie sich mit der Arbeit der Rechtsberater_innen in Nichtregierungsorganisationen im schweizerischen Asylverfahren auseinander.

Küffner, Carla, arbeitet für die Organisation Asyl in Not in Wien in einem international vergleichenden Projekt zum Thema Diskurse zu Fluchthilfe/ Schlepperei und promoviert zum Thema Auseinandersetzungen über Abschiebungen.

Lahusen, Christian, ist Professor für Soziologie an der Universität Siegen. Seine Forschungsschwerpunkte liegen in der politischen Soziologie, der Europasoziologie und der soziologischen Theorie. Er ist Mitantragsteller der von der Deutschen Forschungsgemeinschaft geförderten Forschergruppe »Europäische Vergesellschaftung« und leitet darin ein Projekt zum europäischen Asylverwaltungsfeld gemeinsam mit Karin Schittenhelm.

Laube, Lena, Dr., ist Soziologin und Geschäftsführerin des Forum Internationale Wissenschaft (FIW) der Universität Bonn. Als wissenschaftliche Mitarbeiterin in der Abteilung für Demokratieforschung des FIW forscht und publiziert sie zu Themen der internationalen Visa- und Grenzpolitik, Demokratie und Inklusion sowie zur modernen global vernetzten Politik.

Schneider, Stephanie, ist wissenschaftliche Mitarbeiterin am Seminar für Sozialwissenschaften an der Universität Siegen. In ihrem Promotionsprojekt befasst sie sich mit Auseinandersetzungen über Deutungen »guter Arbeit« im Feld der Asylverwaltung. Ihre Forschungsschwerpunkte liegen in den Bereichen Verwaltung, Recht und Migration.

Wottrich, Kristina, ist Mitarbeiterin bei IN VIA Köln, Stabsstelle Migration und Integration. Nach dem M.Sc. an der Universität Göteborg in Global Studies (Schwerpunkt: Migration Studies) hat sie als wissenschaftliche Mitarbeiterin im DFG-Forschungsprojekt »Auf dem Weg zu einem europäischen Asylverwaltungsfeld?« (Universität Siegen) zur Asylverfahrenspraxis in Schweden geforscht. 


\section{Soziologie}

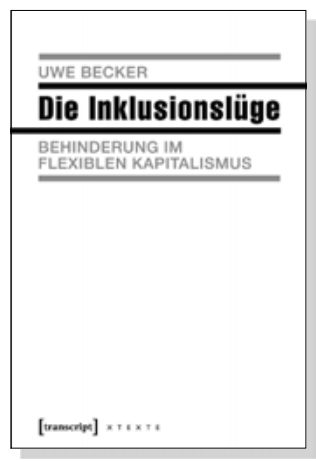

Uwe Becker

Die Inklusionslüge

Behinderung im flexiblen Kapitalismus

2015, 216 S., kart., 19,99 $€(D E)$,

ISBN 978-3-8376-3056-5

E-Book: $17,99 €(\mathrm{DE})$, ISBN $978-3-8394-3056-9$

EPUB: $17,99 €(\mathrm{DE})$, ISBN 978-3-7328-3056-5

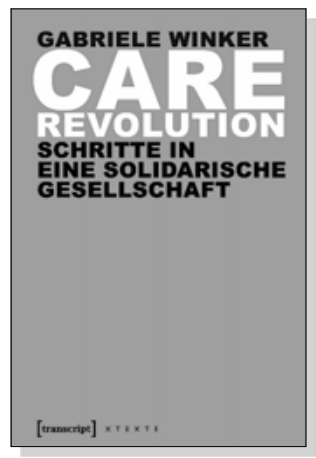

Gabriele Winker

Care Revolution

Schritte in eine solidarische Gesellschaft

2015, 208 S., kart., 11,99€ (DE),

ISBN 978-3-8376-3040-4

E-Book: $10,99 €(D E)$, ISBN 978-3-8394-3040-8

EPUB: $10,99 €(D E)$, ISBN 978-3-7328-3040-4

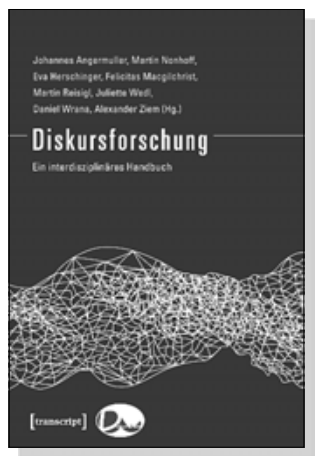

Johannes Angermuller, Martin Nonhoff, Eva Herschinger, Felicitas Macgilchrist, Martin Reisigl, Juliette Wedl, Daniel Wrana, Alexander Ziem (Hg.)

\section{Diskursforschung}

Ein interdisziplinäres Handbuch (2 Bde.)

2014, 1264 S., kart., 2 Bde. im Schuber, zahlr. Abb. $44,99 €(\mathrm{DE})$, ISBN 978-3-8376-2722-0

E-Book: $44,99 €(\mathrm{DE})$, ISBN 978-3-8394-2722-4 


\section{Soziologie}

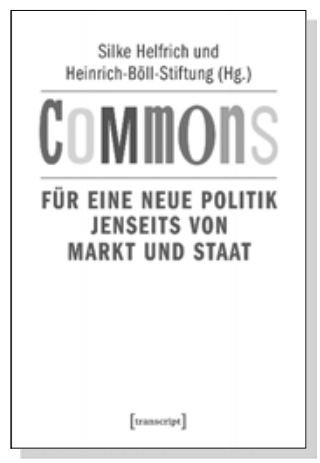

Silke Helfrich, Heinrich-Böll-Stiftung (Hg.)

\section{Commons}

Für eine neue Politik

jenseits von Markt und Staat

2014, 528 S., kart., $24,80 €(D E)$,

ISBN 978-3-8376-2835-7

als Open-Access-Publikation kostenlos erhältlich

E-Book: ISBN 978-3-8394-2835-1

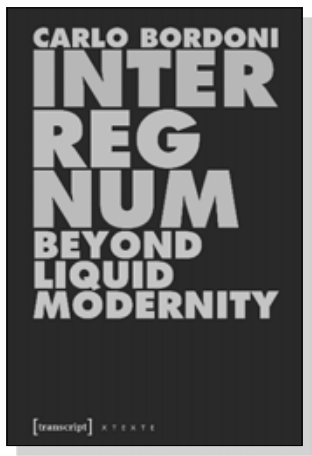

Carlo Bordoni

\section{Interregnum}

Beyond Liquid Modernity

März 2016, 136 p., 19,99€ (DE),

ISBN 978-3-8376-3515-7

E-Book: $17,99 €(\mathrm{DE})$, ISBN $978-3-8394-3515-1$

EPUB: $17,99 €(D E)$, ISBN 978-3-7328-3515-7

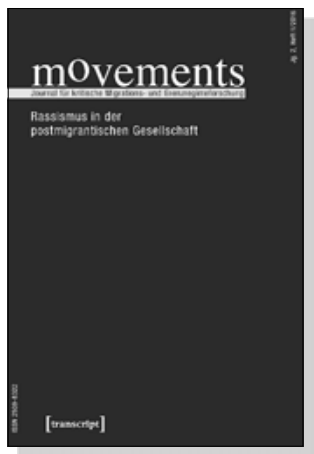

Kijan Espahangizi, Sabine Hess, Juliane Karakayali, Bernd Kasparek, Simona Pagano, Mathias Rodatz, Vassilis S. Tsianos (Hg.) movements. Journal für kritische Migrationsund Grenzregimeforschung

Jg. 2, Heft 1/2016:

Rassismus in der postmigrantischen Gesellschaft

September 2016, 272 S., kart.

24,99€ (DE), ISBN 978-3-8376-3570-6

als Open-Access-Publikation kostenlos erhältlich:

www.movements-journal.org 


\section{Kultur und soziale Praxis bei transcript}

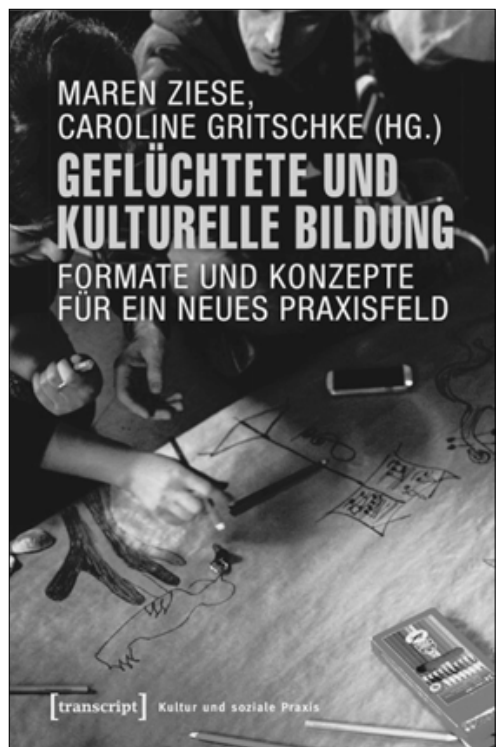

Maren Ziese,

Caroline Gritschke (Hg.)

\section{Geflüchtete und Kulturelle Bildung}

Formate und Konzepte für ein neues Praxisfeld

Oktober 2016, 448 S., kart., $29,99 €$, ISBN 978-3-8376-3453-2

E-Book: $26,99 €$

- Wie können Menschen, die auf der Flucht vor Krieg, Verfolgung, Hunger und wirtschaftlicher Not in Deutschland ankommen, ihre Rechte auf Bildung und gesellschaftliche Teilhabe wahrnehmen? Was sind geeignete Formate der Kulturellen Bildung, um auf die Realität der Geflüchteten aufmerksam zu machen und um Vernetzung und Solidarisierung herzustellen?

Die Beiträge des Bandes gehen diesen Fragen nach und zeigen: Die Kulturelle Bildung ist ein Feld, in dem viele ambitionierte Projekte mit Geflüchteten realisiert werden. Das Phänomen Flucht bietet so Möglichkeiten für eine macht- und differenzsensible Veränderung von Kultur- und Bildungsinstitutionen und eröffnet Chancen für die Revision etablierter Handlungsroutinen.

\section{www.transcript-verlag.de}


